\title{
NBSIR 79-1920
}

\section{A Collection of Test Problems for Discrete Linear $L_{1}$ Data Fitting}

D. R. Shier

S. J. Neupauer

P. B. Saunders

Operations Research Division Center for Applied Mathematics National Engineering Laboratory National Bureau of Standards U.S. Department of Commerce Washington, DC 20234

July 1, 1979

Issued November 1979

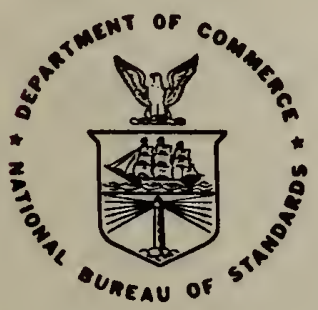

U.S. DEPARTMENT OF COMMERCE

$-Q C-$ NATIONAL BUREAU OF STANDARDS 

Operations Research Division Center for Applied Mathematics National Engineering Laboratory National Bureau of Standards U.S. Department of Commerce Washington, DC 20234

July 1, 1979

Issued November 1979

\section{U.S. DEPARTMENT OF COMMERCE}

Luther H. Hodges, Jr., Under Secretary

Jordan J. Baruch, Assistant Secretary for Science and Technology

NATIONAL BUREAU OF STANDARDS, Ernest Ambler, Director 
$\Delta=5$ 
This document assembles 27 test problems representing a variety of examples in which least absolute deviation (or $\mathrm{L}_{1}$ ) data fitting has been used. The problems were collected from the literature, from the authors of several $L_{1}$ approximation codes, and from examples encountered in practice. Optimal $\mathrm{L}_{1}$ solutions to these problems (objective function value and solution vector) have been obtalned using a double-precision computer code designed for checking the Kuhn-Tucker conditions and for performing an accurate reinversion of the optimal basis.. Special problem characteristics such as alternative optima, degeneracy, and rank loss are also noted. This set of test problems has proven useful in evaluating and improving the performance of $\mathrm{L}_{1}$ codes as well as in suggesting types of problem structures that might be mimicked by problem generators.

Key Words: Algorithm testing; approximation; curve fitting; least absolute deviation; polynomial approximation; spline fitting; test data; test problems 

The authors gratefully acknowledge the support provided by the Computer Services Division, National Bureau of Standards, Washington, DC in allowing use of the UNIVAC 1108 for conducting this research project. 

1. Introduction....................................... 1

2. $\mathrm{L}_{1}$ Data Fltting Problems............................. 3

3. The 27 Hand-Picked Problems......................... 4

4. Verification of Optimal $\mathrm{L}_{1}$ Solutions.................... 8

5. Listing of Input Problem Data.......................11

6. Summary of Problem Solutions.......................... 108

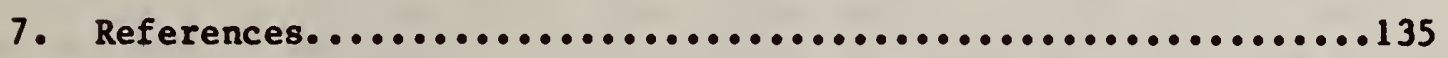





\section{INTRODUCTION}

The fftting of varfous models to existing data and the discrete approximation of given functional relations are tasks frequently encountered in applied mathematics. An extensively studied and used technique in this regard is that of least squares estimation $[9,19,21]$, in which the sum of squared residuals between actual and predicted values is minimized. This approach enjoys a number of useful advantages: the least squares estimates can be found by solving (with appropriate care) a system of linear equations; the statistical properties of such estimators are well established; and, under the assumption of normally distributed errors, a number of statistical tests of hypotheses can be carried out.

In recent years interest has been rekindled in alternative estimation methods, which can be used either in place of or in conjunction with the technique of least squares $[3,10,22,26]$. Of particular interest is the method of least absolute deviation ( or $\mathrm{L}_{1}$ ) data fitting, where the sum of absolute values of the residuals is minimized. This approach also has certain desirable properties: the $\mathrm{L}_{1}$ estimators are maximum likelihood estimators when the errors derive from a Laplace distribution [22]; they can be more efficient than least squares estimators $[22,23]$; they are "robust" with respect to long-tailed distributions $[13,14]$ and do not give undue weight to observations which represent outliers. Moreover, with the advent of reasonably efficient procedures for constrained optimization (descent methods, linear programming), obtaining such estimates is computationally feasible.

A number of algorithms for $\mathrm{L}_{1}$ data fitting problems have been recently advanced $[1,2,5,6,24,25]$, and preliminary comparative testing has been performed at the National Bureau of Standards using four generally available $\mathrm{L}_{1}$ computer codes [12]. Controlled experiments with these codes are facilitated by the avallability of well-defined classes of test problems so that results obtained by sampling a finite number of test problems from a class will possess some measure of validity. For this reason, generators for problem classes with controllable characteristics have been developed [8,16] and used in performing comparisons of $\mathrm{L}_{1}$ codes [12]. In addition, a set of 27 handpicked problems was assembled and employed in an evaluation of the $\mathrm{L}_{1}$ codes. It is significant that although all four codes "correctly" solved each generated problem within certain classes, this was decidedly not the case for the 27 hand-picked problems.

It is belleved, therefore, that specifying these 27 problems in some detail would be a useful service both to code developers and to those involved in testing $\mathrm{L}_{1}$ codes. That is the purpose of this document. In addition to presenting the data values themselves, we also report relevant problem characteristics (e.g. degeneracy, rank deficiency, uniqueness of solution) and verified solution values. This material should allow code developers to perform an initial benchmarking of their procedures and to identify possible areas for algorithmic enhancement. In addition, the problems described here may suggest types of problem structures to be mimicked by problem generators $[8,16,17$, 19]. 
Section 2 defines the general $L_{1}$ data fitting problem and reviews certain relevant features of such problems. Section 3 discusses the selection of the 27 hand-picked problems and documents the format of these problems as well as their solutions. The procedure used for verifying optimal solutions to the test problems is described in Section 4. Individual data values are tabulated in Section 5, and verified solutions are given in Section 6. 


\section{2. $\mathrm{L}_{1}$ DATA FITTING PROBLEMS}

Given $\mathrm{n}$ sets of observations on one dependent variable $\mathrm{y}$ and $\mathrm{m}$ independent variables $x_{1}, \ldots, x_{m}$, the discrete linear $L_{1}$ data fitting problem is that of determining $\beta_{1}, \ldots, \beta_{m}$ to minimize

$$
\phi\left(\beta_{1}, \ldots, \beta_{m}\right)=\sum_{i=1}^{n}\left|y_{i}-\sum_{j=1}^{m} \beta_{j} x_{i j}\right| .
$$

Here, $y_{i}$ refers to the $i-t h$ observation on variable $y$, and $x_{i j}$ refers to the $i-t h$ observation on variable $x_{j}$. In other words, given $n$ observations we wish to estimate $m \leq n$ parameters by minimizing the sum of the absolute values of the residuals

$$
e_{i}=e_{i}\left(\beta_{1}, \ldots, \beta_{m}\right)=y_{i}-\sum_{j=1}^{m} \beta_{j} x_{i j} .
$$

A set of $\beta_{1}^{*}, \ldots, \beta_{m}^{*}$ for which

$$
\phi^{*}=\phi_{1}^{\left(\beta^{*}, \ldots, \beta_{m}^{*}\right)}=\min \left\{\phi\left(\beta_{1}, \ldots, \beta_{m}\right): \quad \beta j \varepsilon R ; j=1, \ldots, m\right\}
$$

is termed an optimal solution vector to the $L_{1}$ data fitting problem, and $\phi^{*}$ is termed the optimal objective function value.

Clearly, the problem data can be represented using an $\mathrm{n} x \mathrm{l}$ column vector $\underline{\mathrm{y}}$ and an $n \times m$ design matrix $X$. The rank of matrix $X$ is denoted by $r \leq m$; $a$ given $\mathrm{L}_{1}$ problem has full rank if $\mathrm{r}=\mathrm{m}$ and has rank loss $\mathrm{m}-\mathrm{r}$ other

Unlike the case for least squares $\left(L_{2}\right)$ approximation, the optimal $L_{1}$ solution vector may not be unique, even when the problem has full rank. In the presence of rank loss, the optimal solution vector will definitely not be unique. It is known $[4,11]$ that an optimal solution vector can always be found that interpolates at least $r$ of the data points: that is, at least $r$ residuals are identically zero at the solution. For our purposes, the problem is said to exhibit degeneracy if $z>r$ residuals are zero at an optimal solution, with degree of degeneracy $z-r$.

A basic solution for the $L_{1}$ data fitting problem is obtained by selecting $r$ data points to be interpolated exactly--data points corresponding to $r$ independent rows (observations) of the design matrix are chosen to have zero residuals. A number of algorithms for solving discrete $L_{1}$ data fitting problems examine only these basic solutions and proceed in a systematic fashion from one basic solution to the next. A collection of $r$ rows from $X$ that defines a basic solution is said to constitute a set of $r$ active contraints. In the presence of degeneracy, the same optimal solution vector will correspond to several selections of active constraints, or basic solutions. If the problem has full rank, then a unique set $\left\{\beta_{1}, \ldots, \beta_{\mathrm{m}}\right\}$ is determined by selecting any $\mathrm{r}=\mathrm{m}$ constraints to be active. 
The 27 problems compiled and studied in this report were obtained in the following ways.

1. By selecting problems described or solved in journal articles or technical reports

2. By soliciting test problems from the authors of various $\mathrm{L}_{1}$ data fitting codes 1

3. By collecting problems from actual applications

Table 1 shows various sources for the hand-picked problems categorized by these three headings. In addition, the problems can be roughly classified into the following structural types.

1. General data fitting problems

2. Spline-fitting problems

3. Polynomial approximation problems

4. Others

The disposition of the 27 hand-picked problems by these problem types is shown in Table 2.

We have provided listings of the input data for al1 27 problems in Section 5. The first line is a header which gives a brief identification of the problem. The next line gives the values for $n$ (the number of observations) and $m$ (the number of parammeters) in FORTRAN format (2I5). The succeeding.1ines contain the input data for successive observation sets $\left(y_{1}, x_{1}, \ldots, x_{1 m}\right)$ in FORTRAN format (5E 15.7).

Input values are given to seven significant figures when necessary. Because conversion of real numbers to the internal representation of any computer generally involves a round-off or truncation error, the given (single-precision) input values may define different internally represented $\mathrm{L}_{1}$ problems for different machines. The choice of seven significant decimal digits was made to enhance the "portability" of such problems to other machines. Our current study, it should be noted, was performed on the National Bureau of Standards' UNIVAC 1108, Exec 8 operating System. On this computer, single-precision floating-point numbers are represented using a 36-bit word, with one sign bit, eight exponent bits and twenty-seven mantissa bits (approximately 8.1 decimal digits).

IVe are especially indebted to N. Abdelmalek and R. Bartels for a number of these test problems. 
Table 1

Sources of the 27 Hand-Picked Problems

\begin{tabular}{|l|l|l|}
\hline Articles & \multicolumn{1}{|c|}{ Authors } & Applications \\
\hline HP1 & HP3 & HP4 \\
HP2 & HP8-HP26 & HP27 \\
HP6 & & \\
HP7 & \\
& & \\
& & \\
\hline
\end{tabular}


Table 2

Structural Types for the 27 Hand-Picked Problems

\begin{tabular}{|l|l|l|l|}
\hline \multicolumn{1}{|c|}{ General } & Sp1ine & Polynomial & \multicolumn{1}{|c|}{ Other } \\
\hline HP4 & HP16 & HP1-HP3 & HP5* \\
HP15 & HP27 & HP6-HP14 & HP21-HP24** \\
& & HP17-HP20 & \\
& & & \\
& & & \\
& & & \\
\hline
\end{tabular}

* Matrix Inversion

** Trigonometric Approximation 
Section 6 contains a summary, for each problem of its source, its characteristics, and its verified solution(s). Each of the items in this summary is discussed in turn.

1. Identification. Each problem is assigned a unique identifying code number (HP1-HP27).

2. Source. A brief reference is provided to an article discussing the problem, or to the type of application giving rise to the problem.

3. Characteristics. Various characteristics of the input problem are given, including the number of parameters (m), the number of observations ( $n$ ), and the rank of the design matrix.

4. Solution. An optimal set of active constraints and associated numerical solution values are provided as follows.

(a) the (unique) optimal objective function value $\phi *$ is given, accurate to the number of significant digits shown.

(b) A set of active constraints defining an optimal solution vector is given. In the case of degeneracy, alternative sets of active constraints defining the same solution vector are indicated.

(c) Numerical values for the solution vector $\beta_{*}^{*}=\left(\begin{array}{l}\beta * \\ 1\end{array}, \ldots, \frac{\beta *}{\mathrm{~m}}\right)$ are given, accurate to the number of significant digits shown.

(d) An estimate of the 1-condition number $[7,20]$ of the final basis matrix $B$ defined by the set of active constraints. Since the solution vector $\beta *$ is obtained by solving a linear system with coefficient matrix $B$, the 1 -condition number provides information on the accuracy obtainable in $\underline{\beta} *$ from the input data (accurate to seven decimal digits). Namely, if this quantity is approximately $10^{\mathrm{d}}$, then the components of $\underline{\beta}$ can usually be expected to have $\mathrm{d}$ fewer significant figures of accuracy than the elements of $B$ [7].

(e) An indicator is given of whether or not the problem possesses a unique optimal solution vector $\underline{\beta}^{*}$. If there exist alternative optimal solution vectors to the probvlem (in which case there are infinitely many, since the set of solution vectors forms a convex set), then another optimal basic solution vector is given, together with its defining set of active constraints and the associated condition number.

Solutions are given, when possible, as simple fractions. values are provided to seven significant decimal digits. Otherwise, solution Optinal solutions were verified by means of a computer routine designed to check the Kuhn-Tucker conditions of an associated linear program. (Details of the procedure used to verify optimal solutions are provided in Section 4.) Essentially, for a given set of active contraints, the routine calculates (in double-precision) both the solution vector $\underline{\beta}$ and the objective function value $\phi$. It is then determined whether or not this set of active contstraints, and the corresponding solution vector, do indeed represent an optimal solution to the given $\mathrm{L}_{1}$ problem. 


\section{VERIFICATION OF OPT IMAL L $\mathrm{L}_{1}$ SOLUTIONS}

In this section, we describe a method for verifying whether a purported solution to a discrete linear $\mathrm{L}_{1}$ data fitting problem is in fact an optimal solution. Recall that the problem of interest is that of determining $\beta_{1}, \ldots, \beta_{\mathrm{m}}$ to

(1) minimize $\sum_{i=1}^{n}\left|y_{i}-\sum_{j=1}^{m} \beta_{j} x_{i j}\right|$.

Without loss of generality, we can assume that the matrix $x=\left(x_{i j}\right)$ has rank $r \leq m$ and that the first $r$ columns of $X$ are linearly independent. Since an optimal solution can be obtained with $\beta_{j}=0$ for $j>r$, the original $L_{l}$ approximation problem can be formulated [16] as the following linear program:

minimize

$$
\sum_{i=1}^{n} \alpha_{i}
$$

subject to

$$
\begin{aligned}
& \alpha_{i}+\sum_{j=1}^{r} \beta_{j} x_{i j} \geq y_{i}, \quad i=1, \ldots, n, \\
& \alpha_{i}-\sum_{j=1}^{r} \beta_{j} x_{i j} \geq-y_{i}, \quad i=1, \ldots, n,
\end{aligned}
$$

where $\alpha_{i}$ represents the absolute value of the $i-t h$ residual

$$
e_{i}=y_{i}-\sum_{j=1}^{r} \beta_{j} x_{i j}
$$

Necessary and sufficient conditions for $\alpha_{1}^{*}, \ldots, \alpha_{\mathrm{n}}^{*}, \beta_{1}^{*}, \ldots, \beta_{\mathrm{r}}^{*}$ to be an optimal solution to the above problem are provided by the Kuhn-Tucker conditions [1.5]: there must exist $u_{1}, \ldots, u_{n}, v_{1}, \ldots, v_{n}$ such that

$$
\alpha_{i}^{*}+\sum_{j=1}^{r} \beta_{j}^{*} x_{i j} \geq y_{i}, \quad i=1, \ldots, n
$$

$$
\alpha_{i}^{*}-\sum_{j=1}^{r} \beta_{j}^{*} x_{i j} \geq-y_{i}, \quad i=1, \ldots, n,
$$

$$
u_{i}+v_{i}=1, \quad i=1, \ldots, n \text {, }
$$


$(2.4)$

$$
\sum_{i=1}^{n}\left(u_{i}-v_{i}\right) x_{i j}=0, \quad j=1, \ldots, r,
$$

$$
u_{i}\left[\alpha^{*}+\sum_{j=1}^{r} \beta{ }_{j}^{*} x_{i j}-y_{i}\right]=0, \quad i=1, \ldots, n,
$$

$$
v_{i}\left[\begin{array}{c}
\alpha^{*}-\sum_{j=1}^{r} \beta_{j}^{*} x_{i j}+y_{i} \\
i
\end{array}\right]=0, \quad i=1, \ldots, n \text {, }
$$

$$
u_{i}, v_{i} \geq 0, \quad i=1, \ldots, n
$$

These conditions can be considerably simplified and then applied to judge whether a given $\underline{B}=\left(\beta_{1}, \ldots, \beta_{r}\right)$ represents an optimal $L_{1}$ solution to ( 1$)$. In order to achieve this simplification, let us partition, for a given $\underline{B}$, the index set $\mathrm{N}=\{1, \ldots, \mathrm{n}\}$ into three dis joint subsets $\mathrm{N}^{+}, \mathrm{N}^{-}, \mathrm{N}^{0}$ :

$$
\begin{aligned}
& \mathrm{N}^{+}=\left\{i \varepsilon \mathrm{N}: \mathrm{e}_{i}>0\right\}, \\
& \mathrm{N}^{-}=\left\{i \varepsilon N: \mathrm{e}_{i}<0\right\}, \\
& \mathrm{N}^{0}=\left\{i \varepsilon \mathrm{N}: \mathrm{e}_{i}=0\right\} .
\end{aligned}
$$

When inequalities (2.1) and (2.2) are sumned for fixed $i$, we obtain $\alpha^{*} \geq 0$. Thus, if $i \varepsilon N$ (so that $e_{i}>0$ ), then $\underset{i}{\alpha *}+e_{i}>0$. From (2.6) it follows that $\mathrm{v}_{\mathrm{i}}=0$ for $i \varepsilon \mathrm{N}^{+}$, $\underset{i}{\text { and }}$ from (2.3) that $\mathrm{u}_{\mathrm{i}}=1$. In a similar fashion, if $\mathrm{i} \varepsilon \mathrm{N}$ then $e_{i}<0$ and $\underset{i}{\alpha}-e_{i}>0$, whence using (2.5) and (2.3) we obtain $u_{i}=0$ and $v_{i}=1$.

Equation (2.4) can be rewritten as

$$
\sum_{i \in N^{+}}\left(u_{i}-v_{i}\right) x_{i j}+\sum_{i \varepsilon N^{-}}\left(u_{i}-v_{i}\right) x_{i j}+\sum_{i \in N^{0}}\left(u_{i}-v_{i}\right) x_{i j}=0
$$

or, using the above values for $u_{i}$ and $v_{i}$,

$$
\sum_{i \in N^{+}} x_{i j}-\sum_{i \varepsilon N^{-}} x_{i j}+\sum_{i \in N^{0}}\left(u_{i}-v_{i}\right) x_{i j}=0 .
$$


This latter relation can be recast, via (2.3), as

(3) $\sum_{i \varepsilon N} 0^{u_{i} x_{i j}}=1 / 2\left\{\sum_{i \varepsilon N^{-}} x_{i j}+\sum_{i \varepsilon N} 0^{x_{i j}}-\sum_{i \varepsilon N^{+}} x_{i j}\right\}, \quad j=1, \ldots, r$.

Recall that for given values $\beta_{1}, \ldots \beta_{r}$ we have $\alpha_{i}=\left|y_{i}-\sum_{i=1}^{r} \beta_{j} x_{1 j}\right|$,

$i=1, \ldots, n$. Then conditions (2.1) and (2.2) are automatically satisfied, and the remaining conditions obtain if and only if there exist values $\underline{u}=$ $\left(u_{1}, \ldots, u_{n}\right)$ that satisfy equation ( 3 ) and

(4) $0 \leq u_{i} \leq 1, \quad i=1, \ldots, n$.

The relations of (3) simply represent a linear system $u B=c$ of $r$ equations in $z$ $=\left|N^{0}\right|$ unknowns. In the absence of degeneracy $(r=z)$ and if matrix $B$ is nonsingular, then equation (3) can be solved explicitly as $\underline{u}=\mathrm{cB}^{-1}$ and condition (4) can be readily checked. In the cases when either $B$ is singular or $z>r$ (degeneracy), we can determine whether there is a feasible solution to (3) and (4) by using linear programming. A "Phase I" calculation can be readily carried out using artificial variables [15] to discover whether the following system does indeed admit a feasible solution:

$$
\begin{aligned}
& \underline{u} B=\underline{c}, \\
& \underline{u} \leq \underline{1}, \\
& \underline{u} \geq \underline{0} .
\end{aligned}
$$

In the computer program used to implement these ideas, the data values $y$ and $X$ are input, together with a candidate set of $r$ active constraints $\left\{i_{1}, \ldots, i_{r}\right\}$, associated with independent rows of the design matrix. The associated solution vector $\underline{\beta}$ is then obtalned by solving in double-precision the (nonsingular) system $A \underline{B}=\underline{y}$, where $A=\left(x_{i k}, j\right)$ is the $r \times r$ submatrix corresponding to the active constraint rows and the first $r$ columns of $x$. From these $\beta_{j}$ values, the residuals $e_{i}(i=1, \ldots, n)$ are determined and the

index sets $\mathrm{N}^{+}, \mathrm{N}^{-}, \mathrm{N}^{0}$ are obtained. At this point the corresponding objective function value

$$
\phi\left(\beta_{1}, \ldots, \beta_{r}\right)=\sum_{i=1}^{n}\left|e_{i}\right|
$$

is also calculated in double-precision. Finally, the existence of values $u_{i}$ that satisfy ( 3 ) and (4) is checked either by direct (double-precision) solution of $\underline{u B}=c$ or by applying a revised simplex computer code to system (5). If such values exist (to within pre-specified tolerances), then $\left\{i_{1}, \ldots, i_{r}\right\}$ is deemed an optimal set of active constraints, with optimal solution vector $\underline{\beta}$ and optimal objective' function value $\phi$. Otherwise, the candidate solution is reported as nonoptimal, to working precision. 
5. LISTING OF INPUT PROBLEM DATA

HPI : PCLYNEVIAL APORJXIMATISN OREBLEM

5 $.1003000+01 \quad .10000(1)+01 \quad \cdot 1000503+01$

$.1000000+01$

- $1300000+01$

- $2000000+01$

- ‘000000+01

- $100(: 000+) 1$

- $300.000+21$

$.30000(10+1) 1$

- $11000 \mathrm{COO}+21$

- 400 v0 0u+01

? $000000+01$

- $10.3000+01$

- $5 \cos 030+21$ 


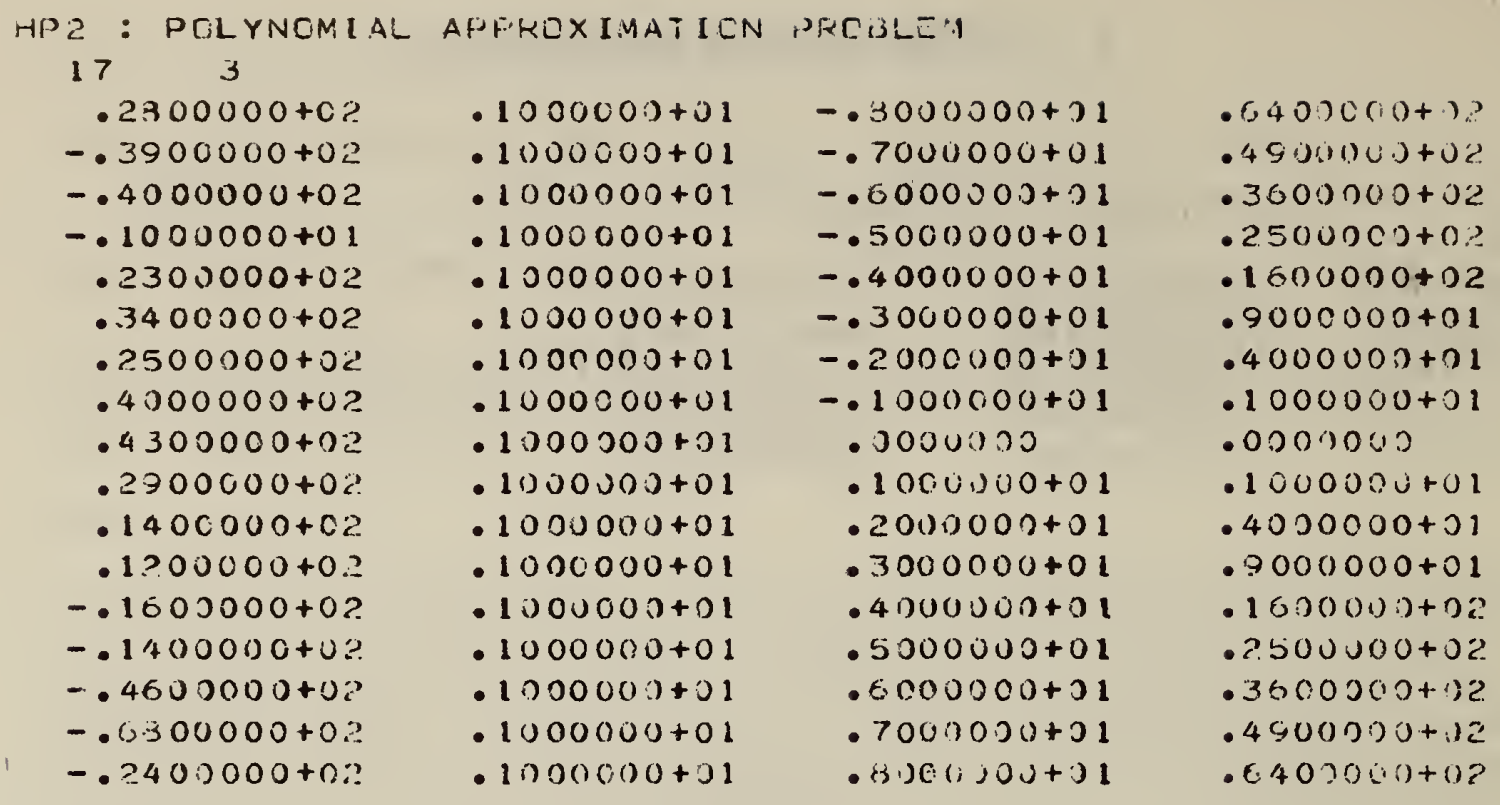


HP3 : POLYHOMIAL- ATORCXIMATIEN PREHLEA

52

$.1140(3)+0.3$

$1000000+1) 1$

$.3501)(0.0)+02$

$.1240000+03$

- $1000000+1$

$.4500 \mathrm{Cr} 0 \mathrm{t}+02$

$.1430000+03$

$.1000600+01$

$.5500000+02$

$.1580001)+0.3$

- $1000000+01$

$.0500000+02$

$.1660000+\mathrm{C} 3$

- $1000000+01$

. 7500000+02 
HP4: GENEPA: DATA FITTING PRLBLEN

1356

$1500000+0 ?$

$.1960001)+03$

$.2600000+02$

. $3610000+03$

$.1700000+02$

- $1960000+03$

- $2500000+02$

- $2560000+03$

- $2300000+02$

- $310000 u+02$

- $2900000+02$

- .3610000+0.3

- $2400000+0$ ?

- 23 $30000+03$

- 27)へ000+02

- $13 \in 000 u+0.3$

- ? $300000+0$ '.

- $1000000+0 \equiv$

- $3500000+02$

- 3610000+03

- $2500000+0 ?$

- $2890000+\mathrm{C}^{3}$

- $2300000+0 ?$

$.144000 n+c 3$

- $2300000+72$

- $1210000+03$

- $3300000+02$

- $3610000+03$

- $2500000+6 ?$

$.4410000+03$

-.3700000+02.

. $5290000+03$

- $3000000+02$

$.1440000+03$

- $2800000+02$

- $1460000+03$

- 231$) 0000+02$

- $1400000+c 2$

- $2500000+02$

- $2560000+03$

- $130000 n+02$

- $14+0000+0 z$

- $1700000+0=$

- $256000 n+1 ; 3$

- 3600000+c2

- $3610200+0.3$

. 2200100+0?

- $1000(i c)+03$

$.2300060+02$.

- $1210000+03$

- $: 400(1)+02$

- $1430000+03$

- $: 200000+02$

- $3100000+0$ ?

- $3: 000 \mathrm{sut}+0$ ?
- $1400 \mathrm{cou}+0 ?$

- $1000000+01$

- $1900000+0 ?$

- $1000000+01$

- $1400000+02$

- $1000000+01$

- $1600000+02$

- 100000 ) +01

- $9000000+01$

- $1000000+01$

- $1900000+02$

- $1000000+01$

- $1700000+02$

- $1000000+01$

- $1400000+02$

- $1000000+01$

- $1000000+102$.

- $1000000+01$

- incocosoto?

- $1000000+01$

- $1700000+0 ?$

- $1000000+i) 1$

- $12.00000+02$

- $1000003+01$

- $1100000+02$

- $1000000+01$

- $19 \mathrm{c} 000$ ) +0 ?

- $1000000+01$

- $2100000+0 ?$

- $100 \mathrm{OUUNO}+01$

- $2300000+0$ ?

- $1000000+01$

- $12000013+02$

- $1300000+01$

- $1400000+0 ?$

- $1000000+01$

- $8000000+01$

- $1000000+01$

- $1600000+0$ ?

- $1000000+01$

- $1200000+52$

- $1000000+01$

- $1600000+02$

- $100000 j+01$

- 19 cocooto?

- $1000(0)+01$

- $10000_{0}+02$

- $1000000+01$

- $1100000+02$

- $10000 \mathrm{C} 0+01$

- $13000 \mathrm{C}$ + 0 ?

- $100(000+3$

- $900000 ; 0+01$

- $1000000+01$

- $2400000+02$

\begin{tabular}{|c|c|c|}
\hline $.1000000+01$ & $.1000000+C 1$ & - () \\
\hline $.1000 C 00+01$ & $.1000000+01$ & .0000000 \\
\hline $.10001000+01$ & $.1000000+01$ & .0000000 \\
\hline $.1000000+01$ & $.1000000+01$ & .0000000 \\
\hline - $1000(100+01$ & $.1000000+01$ & - ก000ก00 \\
\hline $.1000000+01$ & $.1200000+01$ & .0000000 \\
\hline $.1006,000+01$ & $.1000000+21$ & - 100001300 \\
\hline - $10.90(0) 0+01$ & $.10000(1)+01$ & - 1$) 0(0000$ \\
\hline - 100 (1000t01 & $.100000)+01$ & .2000 .000 \\
\hline $.1000006+31$ & $.1000000+01$ & - $x \cos 0 \mathrm{n}$ \\
\hline - $1000004+01$ & $.1000000+11$ & .0003000 \\
\hline $.1000(00+01$ & $.1000000+101$ & .0000000 \\
\hline $.10 \mathrm{~J}: 00+01$ & - $1000000+01$ & . \\
\hline - $1040000+01$ & $.1000000+01$ & .0000000 \\
\hline $.1000000+01$ & $.1000000+01$ & טיט:3000 \\
\hline $.1000000+01$ & $.100000 \mathrm{C}+01$ & $.00 \mathrm{jog}$ \\
\hline $.1000000+01$ & $.1000000+01$ & .0000000 \\
\hline - $1000000+21$ & $.1000000+01$ & .31001000 \\
\hline $.1000000+01$ & $.1000000+01$ & - on.) onc: \\
\hline $.1000000+21$ & $.1000000+01$ & .00).(2000$ \\
\hline $.1000000+01$ & $.1000000+01$ & - consulio \\
\hline - $13 n(j 0100+01$ & $.1000000+01$ & .0001500 \\
\hline $.1000000+01$ & $.1000000+01$ & .0000300 \\
\hline $.1000000+01$ & $.1000000+01$ & . 10000100 \\
\hline $.1000000+01$ & $.1000000+01$ & .0000000 \\
\hline $10(10) 001+71$ & $.1000000+c 1$ & $.000,09$ \\
\hline - $1000000+01$ & $.100000 n+01$ & $.0(0100000$ \\
\hline $100000 c+01$ & $.1000000+01$ & - Si $(10) \%$ \\
\hline
\end{tabular}


$.5760000+03$

. $3400000+02$

$.1210000+03$

. $22000010+02$

. $2560000+03$

- $1100000+0$ ?

$.8100000+02$

- $1700000+02$

. $2560000+03$

- $2100000+02$

- $1960000+03$

- $2800000+02$.

. $3240000+03$

- $2800000+02$

. $3240000+0.3$

. $3300000+02$

$.2560000+0.3$

- $3600003+02$

- $t 2550000+03$

. $3600003+02$

. $4840000+03$

- $2600000+0 ?$

- $1960000+0 j$

$.2400000+c$ ?

. $4000000+03$

- $3010000+02$

- $1440000+03$

$.3800000+02$

$.4410000+03$

$.3200000+02$

$.16,90000+03$

$.3400000+02$

. $3240000+03$

- $2200000+02$

- $1360000+03$

$. ? 700000+02$

$.3610000+03$

. $3700000+02$

. $4410000+03$

. $2900000+02$

$.3240000+03$

. $3300000+02$

- $2560000+03$

- $2100000+02$

. $3010000+03$ $.2500000+02$.

. $289 \mathrm{C000+03}$

$.2500000+02$

$.1900000+03$ $.3500000+02$

- $3240000+03$

- $4200000+02$

$.4840000+03$

- $2700000+0$ ?

- $1000000+03$

- $1800000+02$

- $2830000+03$

. $2.300000+02$
- $1000000+01$

$.1100000+02$

- $1000000+01$

- $1600000+02$

- $1000000+01$

- $9000000+01$

- $1000000+01$

$.1600000+02$

- $1000000+01$

$.1400000+02$

- $1000000+01$

- $1300000+02$

$.1000000+01$

$.180000 j+02$

$.1000000+01$

$.1600000+02$

- $1000000+01$

$.2500000+0 a$.

- $10 \mathrm{Coc00}+01$

$.2200003+0$ r

- $1000000+01$

$.1400001+02$.

- $1000000+01$

- ? J $00000+0$ ?

- $10000(1)+01$

- $1200000+02$

- $1000000+01$

- $2100000+02$

- $1000000+01$

$.1300000+02$

- $1000000+01$

- $1300003+02$

- $1000000+01$

- $1400000+0$ ?

- $1000000+01$

- $1 \rightarrow 00000+0$ ?

- 1 ) $c 0001+01$

- $2100000+0$ ?

- $1.0000 v+01$

- $180000 u+02$

$.1000000+01$

- $1600000+02$

$.1000000+01$

- $1900000+02$

- $1000000+01$

- $1700000+02$

- $1000000+31$

$.1400000+02$

$.1000000+01$

- $1300000+0$ ?

- $1000000+01$

- $2200000+0 ?$

$.100 n 001)+01$

- $1200000+0$ ?

- $1000000+01$

$.1700000+02$

- $1000000+01$

. $1300000+02$
- $1000000+01$

$.1000000+01$

$.1000000+01$

$.1000000+01$

$.1000000+01$

$.1000000+01$

$.1000000+01$

$.1001000+01$

$.1000000+01$

.0000000

.0000000

.0000000

.0000000

.0000000

.0000000

.0000000

.0000000

.0000000

.00100000

.0000000

.00110000

.0000000

.0000000

.0000000

.0000000

.0000000

.0000000

.000 .0000

$.1000000+21$
$.1000000+01$

- ousonon

$1000000+01$

.0030030

$.1000000+01$

.9000000

$.1000000+01$

.0070300

-1000000+01

.0000000

$.1000000+01$

$.0030,000$

$.1000000+01$

.0000002

$.1000000+11$

$.000003 i$

.0000000

$.1000000+01$

$.1000000+01$

.0000033

$.10019000+11$

.0003060

$.1000000+21$

.0500000

$.1000000+01$

.000 .0013

$.1000000+01$

.000070

$.1000000+01$

.0000000

$.1000000+01$

.0000000

$.1000000+01$

.0000000

$.1000000+11$

.000000 a

$.100000 u+01$

.00000000

$.1000000+01$

.0030000

$.1000000+01$

.0000000

$.1000000+21$

.0000000

$.1000000+01$

.0000 .000

$.1000000+11$

.0000000

$.1000000+31$

.0000000

$.1000000+01$

.0000000

$.1000000+1) 1$

.0000000

$.1000000+01$

.0c00000

$-.1000000+01$

$-.1600000+01$ 
$.3240000+03$ . $2800000+02$ . $4000000+03$ - $8000000+01$ $.2500000+02$ - $1600000+02$ - $3240000+03$ - $1200000+02$ . $3100000+02$ . $2100000+02$ $.2550000+03$ $.1900000+02$ - $2560000+0.3$ $.2000000+0{ }^{2}$. - $1960000+03$ $.2400000+02$ $.5760000+03$ $.1700000+02$ . $2560000+0.3$ $.1400000+02$ $.1210000+C 3$ $.1800000+02$ . $2250000+03$ $.1400000+0$ ? $.4410000+C .3$ . $1500000+c 2$ $.1960000+0$ is $.1400000+C 2$ . $4840000+03$ $.1300000+02$ . $2560000+03$ - $1830000+02$ . $8100000+0$ ? - $2700000+02$ - $5250000+0.3$ . $2700000+02$ - $2330000+0.3$ - $200000.3+02$ ? - ir 30 or. $00+0.3$ - la $00000+c 2$ $. \$ 400000+02$ $.1400000+C^{2}$ - 1440703+63 . $2100000+02$ - ‘fs $90000+0.3$ $.3500000+02$ $.40200000+03$ - $300000+152$ . $3240000+03$ - $1200000+0$ ? - $1960000+03$ $.1300000+c 2$ - 1690000+03 . $2200500+0^{2}$ - $2330: 500+03$ $.1300000+02$ - 22:c,0000+03 - $1100 \cos +c$.
- $1000000+01$ $.2000000+02$ - $1000000+01$ $.5000000+01$ $.1000000+01$ - $1360000+02$ $.1000000+01$ $.9000000+01$ - $1000000+01$ - $1600000+02$ $.1000000+01$ $.1600000+0$ ? . 1200001$)+01$ $.1400000+02$ $.1000000+01$ $.24000 v 0+02$ $.1000000+11$

- $1 € 00000+02$ - $1200000+01$ - $1100000+02$ $.1000000+01$ $.1500000+02$ - $1000000+01$ $.2100000+02$ - $1000 r, 00+31$ $.1400000+02$ - 1 ucoojot j1 - 22000000+0? - $1000000+01$ - $1600000+02$ - $1000000+01$ -9c00000+01 - 11) $00000+01$ - $2300000+02$ - $1000000+01$ $.1700000+02$ - $1000000+01$ - $1700000+02$ - $1000000+01$ $.8000000+01$ - $1000000+01$ - $1200000+02$ - $1300000+01$ - $1700010+02$ - 1$) 00000+01$ - $2000600+02$ - $1000000+01$ - 1 300coutos - $1000000+01$ - $1400000+02$ - $1300000+01$ - $1300000+02$ - $1000000+01$ - $17.00003+02$ - $10 \cos 00+01$ - $1500000+02$ - 1 . J cocosotol - 13 sucouto:
$.1000000+01$

$-.1000000+01$

$-.1000000+31$

$.1000000+01$

$.1000000+01$

$.1000000+01$

$.1000050+01$

- $1000000+01$

$.1000000+01$

$.1000000+01$

$.1000000+01$

- 1 oriujonto 1

$.1000000+01$

$.1000000+01$

-1000000+01 :

$.1000000+131$

- $1000000+01$

$100000+01$

$.1000000+01$

$.1000000+01$

$.1000000+01$

- $100 p 000+01$

$.1000002+? 1$

-120ษั

- $100(3)(19) 0+01$

$-1000000+01$

- $1006020+01$

$-.1000000+01$

$.1000000+01$

$-.1000030+21$

$.1000000+01$

$-.10000 .00+01$

$.100000)+01$

$-.1000000+01$

- 1000.2301

.0000000

-0ovasos
$-.1000003+01$

$-.1000000+01$

$-.1000000+01$

$-.1000000+01$

$-.1000000+01$

$-.1002000+01$

$-.1000000+01$

$-.1000000+01$

$-.1000000+01$

$-.1000000+01$

$-.1000000+c 1$

$-.1 .000002+01$

$-.1000000+01$

$-.1000000+01$

$-.1000000+01$

$-.1000000+31$

$-.1000000+01$

$-.1000000+01$

$-.1000000+01$

$-.10,0000)+11$

$-.1000000+01$

$-.1000000+01$

$-.1000000+11$

$-.1000000+11$

$-.1000000+01$

$-.10 .30 .000+01$

$-.1 .300000+01$

$-.1000000+01$

$-.10(9000+1) 1$

$-.1000002+01$

$-.1000000+01$

$-.1000000+01$

$-.1000000+01$

$-.13 n \sin 2+21$

$-.1000003+.1$

$-.1000000+01$

$-.1000) 0 \%+1$

$-.1000000+11$

$-.107000 .3+31$

$-.1000000+01$

$-.(1.3920)+11$

$-.1(1,1,1 \%,+1) !$

$-.16 .11: 2914: 1$

$-.1(00020+11$

$-.1000000+01$

$-.1010000+: 1$

$-.1000000+1$

$-.1(2 ! \cdot) \cdot 3)(3+.1$

$-.10 .000: 1+101$

$-.1000(916+1) 1 \quad-.10(i 0): 3+3 i$ 
$. .3240000+03$

$.3700000+02$

$.7290000+03$

. $2100000+02$

- $1210000+03$

$.1700000+02$

$.1690000+03$

$.1700000+02$

- $2250000+0.3$

. $2600000+02$

$.1690000+03$

$.2100000+02$

$.1000000+03$

$.3700000+02$

$.4410000+03$

$.1100000+02$.

- $1590000+03$

$.1400000+02$

$.1000000+03$

$.1700000+02$

$.2390000+03$

. $3000000+02$

$.4840000+03$

. ?000000+0:

- $16.40000+c 3$

- $1200000+02$

- $1210000+0$. .

- $1600000+02$

- $2250000+03$

- $3300000+02$

. $4840000+03$

$.1600000+0$ ?

$.1690000+05$

$.8000000+01$

$.8100000+02$

- $2.300 \mathrm{c} 00+0$ ?

. $32.40000+03$

$.1700000+02$

- $1210000+03$

. $2100000+02$

- $1440000+03$

. $1200000+02$

- $2560000+03$

. $2300000+02$

- $1210000+C 3$

- 3000000+0a.

. $4410000+03$

- $1500000+0$ ?

- $8100000+02$

- $1500000+02$

- $1440000+03$

- $2400000+02$

- $5250000+03$

$.1800000+02$

. $1210000+03$

$.2700000+02$

$.5700000+0.3$

- $000000+01$
- $1000000+01$

. $2700000+02$

. $1000000+01$

- $1100000+02$

- $1000000+01$

$.1300000+02$

- $1000000+01$

- $1500000+02$

$.1000000+01$

- $1300000+0 ?$

$.1000000+01$

$.1000000+02$

- $1000000+01$

$.2100000+02$

- $1060000+01$

$.1300000+0$ ?

- $1000000+01$

- $1000000+02$

$.1000000+01$

$.1700000+0 a$

- $1000000+01$

- á20000u+0a

- $1000600+01$

- $1300000+02$

- $1000000+01$

- $110000 u+02$

- $1000000+01$

- $1500000+02$

- $1000000+01$

. $2200000+0 a$

- $1000000+01$

- $1300000+02$

- $1000000+01$

- $5000000+01$

$.1000000+01$

- $1300000+02$

$.1000000+01$

- $1100000+02$

- $1000000+01$

- $1200000+02$

- $1000000+01$

- $1000000+0$ ?

- $1000000+01$

- $1100000+02$

- $1 \leqslant 00000+01$

$.2100000+02$

- $1000000+01$

$.9000000+01$

- $1000000+01$

- $1200000+02$

- $1000000+01$

- $2500 \mathrm{co0}+02$

- $1000000+01$

- $1100000+02$

- $1000000+01$

- 2400000+02

- $1300000+01$

- $1400000+0$ ?
.0000000

$$
-.1000000+01
$$

$-.1000020+31$

.0000000

$-.1000000+01$

$-.1090000+0.1$

.0000000

$1000000+01$

$-.1000000+111$

.0000000

$1000000+01$

.0000000

$1000000+01$

.0000000

$1000000+01$

.0000000

$1000002+01$

.0000000

$1000000+.01$

.0000000

.0000000

.0000000

$.1000000+01$

$.1000000+01$

$.1000000+01$

$.1000000+01$

$.1000000+01$

$.1000000+01$

$.1000000+01$

$.1000000+01$

- $1000000+01$

$.1000000+01$

$.1000000+01$

$.1000000+01$

$.1000000+01$

$.1000000+01$

.0000 .00

.0000090

.0000000

.0000000
$1000000+01$

$.10000100+01$

$-.10000(0+01$

.0000000

.0000000

.0000000

.0000000

.0000000

.0000000

.0000000

.0000000

.0000000

$.000000 c_{i}$

.0000000

.0000000

.00 .00000

.0000000

.0000000

.0000000

.0000000

.0000000
$-.1000000+11$

$-.1000000+01$

$-.1000300+01$

$-.1010030+01$

$-.1000000+11$

$-.1000000+11$

$-.1020000+21$

$-.1700000+i 1$

$.10700(0+1) 1$

$.1000000+01$

$.1000000+n 1$

$.100030+01$

$.1000060+01$

$.1000000+01$

$.1000000+01$

$.1000060+01$

$.1000000+01$

$.1000000+01$

$.1000000+01$

$.1000000+J 1$

$.10000 r 0+21$

$.1000000+1) 1$

-11)00000+31

$.1000000+01$

$.100000 n+01$

$.1020000+21$ 
- $1960000+03$ $.120000 n+02$ $.2560000+0.3$ $.1500000+02$ - $1960000+03$ . $2800000+02$ $.3240000+03$ $.1900000+02$ $.0400000+02$ $.1800000+02$ $.6400000+02$ $.2200000+02$ $.1960000+03$ $.1500000+02$ $.2250001)+03$ $.1700000+02$ - $2560000+03$ $.1300000+02$ $.5100000+02$ $.1400000+02$ $.1440000+03$ - 3300000+02 $.1225000+04$ . $2000000+02$ - $2250000+03$ $.1600000+02$ - $2250000+03$ . $3000000+02$ $. \$ 410000+C 3$ - $1300000+02$ . $8100000+02$ $.2200000+02$ $.4000000+0.3$ . $9000000+01$ - é2 $250000+03$ $.1900000+02$ $.1210000+03$ $.1100000+02$ $.4840000+0.3$ $.3400000+02$ . $5250 \mathrm{OCOO+03}$
$.1000000+01$ $.1600000+02$ - $1060000+01$ - $1400000+02$ - $1000000+01$ - $1300000+02$ - 1) $(0000+i) 1$ $.8000000+01$

- $1000000+01$

- $8000000+01$

- $1000000+01$

- $1400000+0$ ?

- $1000000+01$

- $1500000+02$

$.1000000+01$

$.160008 \mathrm{u}+02^{3}$

- $1000000+01$

- $000 \mathrm{coO}+01$

- $10 \mathrm{cc000+01}$

- $1230000+02$

$.1000000+01$ $.3500000+02$ - $1000000+01$ - $1500000+0$ ? - $1000000+01$ $.15000 .20+02$ - $1000000+01$ . $2100000+02$ - $10 \mathrm{ccc} c 00+01$ - $9000000+01$ - $1000000+01$ - $2000000+02$ - 1 c cocjotol $.1500000+02$ - $1000000+01$

- $1100000+02$ - $1000000+01$ - $2200000+02$ - 10 cocootol - $2300000+02$ $.1000(000+) 1$

\begin{tabular}{|c|c|c|}
\hline .0000000 & .0000000 & $.1000000+01$ \\
\hline .0000000 & .0000000 & $.1000000+01$ \\
\hline .0000000 & .0000000 & $.1000200+01$ \\
\hline .0000000 & .0000000 & $.1000020+01$ \\
\hline .0000000 & .0000000 & $.1000000+11$ \\
\hline .6000000 & .0000000 & $.1000000+31$ \\
\hline .0006000 & - $\operatorname{coc} 0,000$ & $.103000 n+i 1$ \\
\hline $.00(00) 0$ & .0000000 & $.1000100+01$ \\
\hline - cercooo & .0000000 & $.1002(000+01$ \\
\hline $.000(1000$ & .0200060 & $.1003000+01$ \\
\hline .00010000 & - ccionoso & $.1330100+11$ \\
\hline .0000000 & .0000000 & $\cdot 10: 20: 200+1$ \\
\hline$\cdot c 000000$ & $\therefore 0000000$ & $.1000300+01$ \\
\hline .0000000 & .0000000 & $.1000000+01$ \\
\hline .0000000 & .0000000 & $-1000000+? 1$ \\
\hline .0000000 & .0000000 & $.100 .0000+01$ \\
\hline .0000000 & .0000000 & $-1000000+01$ \\
\hline .0000000 & .0000000 & $.1000000+01$ \\
\hline .0000000 & .0000000 & $.10000(10+1) 1$ \\
\hline .00 .00000 & .0000000 & $.1000130+1$ \\
\hline
\end{tabular}


HPS : MATRIX INVERSIEN PREBLE'I

3 8

$.506 .7600+05$ $.4017000+04$

- $1725940+06$

- $1124600+05$ $.6536500+05$ $.3860000+04$

- $7492800+05$ $.5373000+04$ . $7559300+05$ $.83061000+04$ . $3305400+05$ $.9702000+04$ - $1432300+06$ $.1513100+05$ $.7047500+05$ $.50 € 3000+04$
- $5.300000+03$

$.4560000+03$

- $1077000+04$

. $4667000+04$

. $4300000+03$

- $1013000+04$

- $82.20 .000+03$

- 749 coco 03

- $8090000+03$

- $2350000+0.3$

- $1230000+04$

- $2390000+0.3$

- $9940000+03$

. $2180000+04$

. $1040000+04$

- $1323000+04$
. $28056,00+04$

- $10 \ddot{50000+04}$

- $0412000+04$

. 3959000+04

- $1959000+04$

- $206: 4000+04$

- $2369000+04$

- $3064000+04$

- $3250000+04$

- 40 y0000+03

- 55 () 6 . . $0+04$

- $5910000+03$

- $528 \because 000+04$

- $226(1000+04$

- $1667000+04$

- $2335000+04$
- $1367200+05$

- $1075160+05$

- 352.4000+0\%

.6206000+04

- $2323300+05$

. $3139000+04$

. $3572800+05$

$.1547000+05$

- ?. $309800+3$.

- $1465000+04$

$.2774000+05$

- 1050000104

$.4738100+05$

- $8142000+04$

- $15,364403+35$

- 6. $19400.0+04$
$.1142000+05$

$.3105700+05$

$.1259900+05$

$.1730100+05$

.200160 .05

- $2285900+05$

$.+145100+15$

$.1191100+35$ 
HPO : POLYNCMIA! RIPRCXIMATICN PROSLEM

$6 \quad 2$

. $1520000+02 \quad .1000000+01 \quad .0000600$

$.1025000+02 \quad .1000000+01$

- 1 cuovon+01

$.4750000+01$

. $1000000+01$

- $2000000+01$

$.1000000+00$

$-.4750000+01$

- $1000000+01$

$.3000000+01$

-.1005000+0?

- $1000000+01$

$.4000000+01$

$.1000000+01$

. 5 CON10Outol 
HP7 : PCLYNEM! AI APPRCXIMATIIT PRCIULE!

72

$.1000000+01$

$-1000100+01$

$.106 .0000+01$

$.1200000+02$

$.1000000+01$

$.2000000+01$

$.3000000+01$

$.1000000+01$

$.3000000+01$

$.400000 .0+01$

$.1000000+01$

$.4000000+01$

$.5000000+01$

- $1000000+01$

. $5000000+01$

$.1200000+0$ ?

- $1000000+01$

$.6000000+01$

$.7000000+01$

- $1000000+01$

$.7000000+01$ 
HPB : PCLYNCMIAL. APPREXIMATIDN PROBLEM $201 \quad 1$

.0000000

$.1960000-01$

$.1000000+01$

$.3842000-01$

$.1000000+01$

$.5647000-01$

$.1000000+01$

$.7377000-01$

$.1000000+01$

$.9033000-01$

$.1000000+01$

$.1061800+00$

$.1000000+01$

- $1213100+00$

- $1357600+00$

- $1495400+00$

- $1626600+00$

$.1751300+00$

- $1869800+00$

- $1982200+00$

- ?.083700+00

$.2189300+00$

$.2284200+00$

. $2373700+00$

$.1000000+01$

- $1000000+01$

$.1000000+01$

- $1000000+01$

- $1060000+01$

- $1000000+01$

$.1000000+01$

- $1000000+01$

- $1000000+01$

- $1000 u 00+01$

- $1000000+01$

- $1000000+01$

- $1000000+1$

- $1000000+01$

$.1000000+01$

- $1000000+01$

- $1000 \mathrm{cuot01}$

$.1000000+01$

- $1000000+01$

- $1000000+01$

- $1000000+01$

- $1000000+01$

- $1000000+c 1$

$.1000000+01$

- $1000000+01$

- $1000000+01$

- $1000000+01$

- $1000000+01$

$.1000000+01$

- $100000 J+01$

$.1000000+01$

$.1000000+01$

- $1000000+01$

- $1000000+01$

- $1000000+01$

$.10030 .50+01$

- $1000000+01$

- $1000000+01$

- $1000000+01$

- $1000000+01$

- $1000000+01$

$.1000000+01$

- $1200000+01$

$.1000000+01$

- $1000000+01$

- $1000(j) 0+01$

- $100000 i+01$

- $1000000+01$

- 10 cucostol 
. $2300000+00$

. $2936800+C 0$ $.2900000+00$ . $: 384100+00$ . $2341100+12$ - $2807: 011+00$ $.2772500+00$ . $2736900+00$ - $2700600+00$ . $2663600+00$ . $2626000+00$ $.2587800+00$ . $2549000+00$ . $2502800+00$ . $2470100+00$ . $2430100+c 0$ $.2389700+00$ $.2349000+00$ $.2303100+00$ . $2267000+00$ - $2225700+00$ - $218430 \mathrm{~J}+00$ - $2142800+00$ . $2101200+00$ - $2059700+00$ . $2018103+0 \mathrm{C}$ - 197 CCOO+OO

- $1935200+00$

- $18 ? 3000+00$

- $1852600+C 0$

- $1811600+00$

- $1770800+00$

- $1730100+00$

- $1689700+00$

$.1649600+00$

- $1009800+00$

- $1570200+00$

- $1531000+00$

. $1492100+00$

. $1453500+00$

- $1415400+00$

- $137760 \%+00$

- $1340200+00$

- $1303200+00$

- $1266700+00$

$.1230600+00$

. $1195000+00$

. $1159800+00$

$.1125000+00$

$.1090800+00$

$.1057100+00$

$.1023500+0 c$

- $1310000-01$

- $9583000-01$

- $\rightarrow 271000-01$

. $3953000-01$

$.8651000-01$

. $8350000-01$
$.1000000+01$

$.1000000+01$

- $1000000+01$

- $1000000+01$

- $1000000+01$

- $1700000+01$

- $100000 n+01$

- $1000000+01$

$.1000000+01$

- $1000 \mathrm{ClO} 01$

- $1000000+01$

- $1000000+01$

. $1000000+01$

- $1000000+01$

- $1000000+01$

- $1000000+01$

- $1000000+01$

- $1000000+01$

- $1000000+01$

- $1000 \mathrm{cun}+01$

- $1000000+01$

- $1020000+01$

- $1000000+01$

- $1000000+01$

- $10 \mathrm{c000u+01}$

- $1000000+01$

- $1000000+01$

$.1000000+01$

- $1000000+01$

- $1000000+01$

- $1000000+01$

- $1000000+01$

- $1000000+01$

- $1000000+01$

- $1000000+01$

- $10001000+01$

- $1000000+01$

- $1000010+01$

- $1000000+01$

- $1000000+01$

- $1000000+01$

$.1000000+01$

- $1000000+01$

- $1000000+01$

- $1000000+01$

- $1000000+01$

$.1000000+01$

$.1000000+01$

$.10000 C_{0}+01$

- $1000000+01$

- $1000000+01$

- $1000000+01$

- $100,0000+01$

- $1001000+01$

- $1000000+01$

. $1000000+01$

- $1000000+01$

$.1000000+01$ 


\begin{tabular}{|c|c|}
\hline - & $.1000000+01$ \\
\hline $.7702000-01$ & $.10000 v 0+01$ \\
\hline $.7476,000-01$ & $.1000000+01$ \\
\hline $.7195000-01$ & $.1000000+01$ \\
\hline $.5321000-01$ & $.1000000+01$ \\
\hline . $6651000-01$ & $.1200000+01$ \\
\hline $.6387000-01$ & $.1000000+01$ \\
\hline $.6128000-01$ & $.1000000+01$ \\
\hline $.5374000-01$ & $.1000000+01$ \\
\hline $.5625000-01$ & $.1000000+01$ \\
\hline $.5333000-01$ & $.1000000+01$ \\
\hline $.5145000-01$ & $.1000000+01$ \\
\hline $.4913000-01$ & $.1200000+01$ \\
\hline . $46850 \mathrm{C} 0-01$ & - $1000000+01$ \\
\hline $.4463000-01$ & $.1000000+01$ \\
\hline $.4247000-01$ & . 1:00000+01 \\
\hline $.4035000-01$ & $.1000000+01$ \\
\hline . $3829000-01$ & $.1000000+01$ \\
\hline $.3627000-01$ & $-1000000+01$ \\
\hline $.3431000-01$ & .10000 \\
\hline $.3240000-01$ & $.1000000+01$ \\
\hline $.3054000-01$ & $.1000(100+01$ \\
\hline $.287200 .0-01$ & $.1000000+01$ \\
\hline $.26960013-01$ & - $10000(0+01$ \\
\hline $.2524000-01$ & $.1000000+01$ \\
\hline . $2357000-01$ & $.1000000+01$ \\
\hline $.2195000-01$ & - $10 \cos , c u+u 1$ \\
\hline $.2037000-01$ & - 1200$) 00+01$ \\
\hline $.1 \varepsilon 84000-01$ & $.1000000+01$ \\
\hline $.1735000-01$ & $.1000000+01$ \\
\hline $.1591000-01$ & $.1000000+01$ \\
\hline $.1452000-01$ & . $1000000+01$ \\
\hline - $131<000-01$ & . $1000000+01$ \\
\hline $.1185000-01$ & $.1000000+01$ \\
\hline $.1059000-01$ & $.11000000+01$ \\
\hline - $1360000-02$ & .10030 \\
\hline $.13170000-02$ & $-10 \mathrm{coc}$ \\
\hline . $7030000-02$ & $.1000000+01$ \\
\hline $.5920,000-02$ & $.1000000+01$ \\
\hline $.4350000-0 ?$ & $.10000 ., 0+01$ \\
\hline $.3820000-0 ?$ & $.1000000+01$ \\
\hline $.2830000-c 2$ & $.1000000+01$ \\
\hline $1870000-0:$ & $.10 \mathrm{COC}$ \\
\hline $.4500000-c .3$ & .10000 \\
\hline $.7000000-04$ & $.1000000+01$ \\
\hline $.7800000-0.3$ & $.1000000+01$ \\
\hline $.1600000-02$ & - $1000000+01$ \\
\hline $.2380000-02$ & - $1000000+01$ \\
\hline $.3130000-0 ?$ & $.1000030+01$ \\
\hline $.3850000-02$ & $.1000000+01$ \\
\hline $.4530000-0 ?$ & $.1000000+01$ \\
\hline $.5130000-02$ & - $10 \mathrm{coOCO}+01$ \\
\hline $.5820000-02$ & - $13 \cos 000+3$ \\
\hline - $64 ? 00(0-0 ?$ & - $100000 u+01$ \\
\hline $90000-0 \%$ & $.1000000+01$ \\
\hline $30000-62$ & - $1000000+01$ \\
\hline$C: \operatorname{coc}$ & - 1$)(n 001)+01$ \\
\hline & - $15 \cos (1)(0)+1)$ \\
\hline
\end{tabular}


-.899 c000-0?

-.9430000-0?

-.9840000-02

-.1023000-01

-.1059000-01

$-.1094000-01$

-.1126000-01

-.1155000-01

-.1183000-01

-.1209000-01

-.1233000-01

-.1255000-01

-.127500 0-01

-. $1293000-01$

-.1310000-01

-.1325000-01

-.1338000-01

-.1350000-01

-.1360000-01

-.136.7000-01

-.1376000-01

$-.1382000-01$

$-.1337000-01$

$-.1390000-01$

-.1332000-01

$-.1393000-01$

-.1393000-01

-.1392000-01

-.1339000-01

-.1386000-01
$.1000000+01$

- $1000000+01$

$.1000000+01$

$.1000300+01$

- $1000000+01$

$.1000000+01$

- $1000000+01$

- $1000000+01$

$.1000000+01$

$.1000000+01$

$.1000000+01$

- $1000000+01$

- $1000000+01$

$.1000000+01$

$.1000000+01$

- $1000000+01$

$.1000000+01$

- $1000000+01$

- $1000000+01$

- $1000000+01$

- $1000000+01$

- $1100000+01$

- $1000000+01$

$.1000000+01$

- $1000000+01$

- $1000000+01$

- $1000000+01$

- $1000000+01$

- $1000000+01$

- $1000000+01$ 


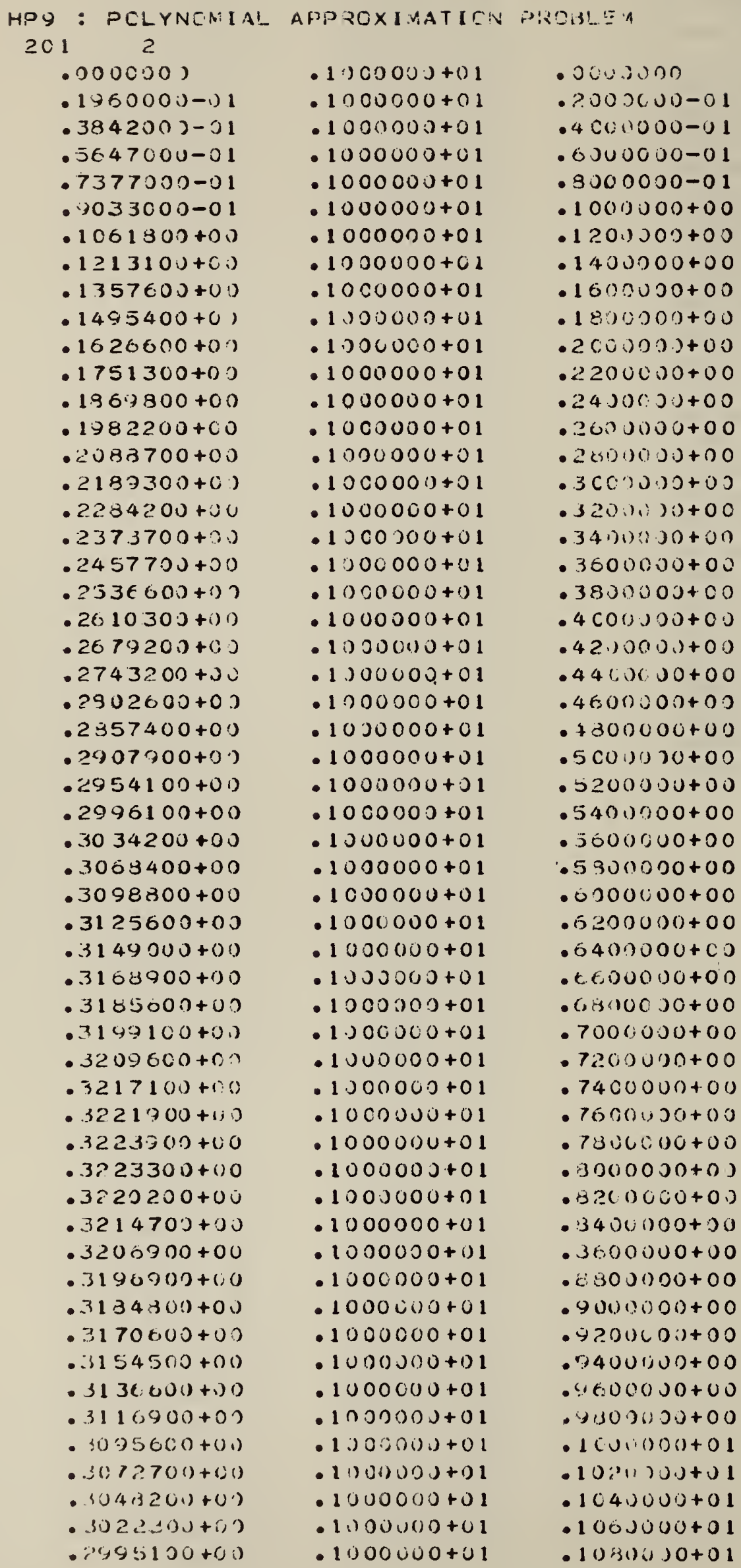


$.2966600+6.0$

. 2936800+o?

- $2906000+00$

- $3874100+00$

- $28.41100+0$ J

- $2307200+00$

. $2772500+00$

$.2736900+00$

$.2700600+00$

. $2663600+00$

. $2620000+00$

$.2587800+00$

. $2549000+00$

- $2509800+00$

. $2470100+00$

. $2430100+00$

. $2389700+0$ )

-2:349000+00

$.2303100+0)$

- $2.267000+00$

- $2225700+00$

- $2184300+00$

. $2142800+00$

. $2101200+00$

$.2059700+00$

. $20181<0+00$

- $1 \rightarrow 76: 600+00$

- $1935230+00$

- $1.393000+00$

- $1852600+00$

- $1811600+00$

- $17708 \mathrm{CO}+00$

$.1730100+00$

. $1689700+00$

$.1649600+00$

- $1609300+00$

. $1570200+00$

- $1531000+00$

$.1492100+00$

$.1453500+00$

$.1415400+00$

. $1377600+00$

- $1340: 00+00$

- $1303200+00$

- $1266700+c 0$

- $1230<00+00$

$.1195000+100$

$.1159800+c 0$

$.1125000+0$ )

$.1040800+00$

$.1057100+00$

$.1023300+c 0$

- $3910000-11$

- 95e $3000-1$

- $1271200-01$

. $\$ 958000-01$

- $35 ! 100 n-01$

. $3350 r: 00-01$
$.1000000+01$

- $1000000+01$

- $1000000+01$

- $1000000+01$

- $1000000+01$

- $1000000+01$

. $1000000+01$

- $1000000+01$

$.1000000+01$

$.1000000+01$

- $1000000+01$

- $1000000+01$

- $1000000+01$

- $1000000+01$

- $1000000+01$

$.1000000+01$

- $1000000+01$

- $1000000+01$

- $1000: 100+01$

- $100 c u c i u+01$

- $1000000+01$

- $1000000+01$

$.1000000+01$

- $1000000+01$

$.1000000+01$

- $1000 u c 0+01$

- $1000000+01$

- $1000000+01$

$.1000000+01$

- $1000000+01$

- $1000000+01$

- $1000000+01$

$.1000000+01$

- $1000000+01$

- $1000000+01$

- 1 juorcutol

- $1000000+01$

- $1000000+01$

- $1000000+01$

- $1000 r u n+01$

- $100000 u+01$

- 1 ococoutur

- $100 c 00 u+01$

- $1000000+01$

- $1000000+01$

- $1000000+01$

- $11000000+01$

- $1000000+01$

- $1000000+01$

$.1000000+01$

$.1000003+01$

$.1000000+01$

- $1000000+01$

- 1 jeccojto 1

- $1300000+01$

$.1000000+01$

$.1000003+01$

$.1000000+v 1$
$.1100000+01$

- $1120000+01$

- $1140000+01$

- $1160000+01$

- $1130000+01$

- $1200000+01$

- $1220000+01$

- $1240000+01$

- $1260400+01$

- $1280000+01$

- $1300000+01$

- 13 3) $00+01$

- $1340000+01$

- $1300000+01$

- $1380000+01$

$.1400000+01$

- $1420000+01$

- $1440000+01$

- $1480 \therefore 0 u+01$

- 14 ह3in. $0 u+01$

- $150(1000+01$

- $1520000+01$

. $15411000+01$

- $1550010+01$

- 1580000101

- $1600(11) 0+01$

. $1620000+01$

. $1640000+01$

- $1660000+01$

- $1630,100+01$

- $170 \mathrm{c} c 00+01$

- $1720000+01$

$.1740000+01$

- $1780000+01$

- $1780000+01$

- $180 n 060+01$

- $13: 20000+01$

- $1840000+01$

- $1860000+01$

- $183 \cos 0 n+01$

- $1900 r i v 0+01$

- $1521(0) 0+01$

- 1540 rinotor 1

- 1960 ar notor

- 19 dou 0001

- $2000000+01$

$.2020000+01$

- $20140000+01$

- $2003(i 00+01$

- $20,10 c \cdot 00+01$

- $2100030+01$

$.2120000+01$

. $2140000+01$

- $2160000+01$

- 21 sisusiot 01

- $2200000+01$

- $232(1 \mathrm{coj} j+01$

- $22 a+u v 0 u+01$ 


\begin{tabular}{|c|c|c|}
\hline .30 & - $1 \cup \operatorname{cov00+01}$ & $\therefore 200000+01$ \\
\hline $.7762000-01$ & - $1000000+01$ & $.2280000+01$ \\
\hline $.7476000-01$ & $\cdot 1000000+01$ & $.2300000+01$ \\
\hline $.7196000-01$ & $.1000000+01$ & $.2320000+01$ \\
\hline $.6921000-01$ & $.1000000+01$ & $.2340000+01$ \\
\hline $.0651000-01$ & $.1000000+01$ & . $2300000+01$ \\
\hline $.6337000-01$ & $.1000000+01$ & $.2380000+01$ \\
\hline $.6128000-01$ & $.1000000+01$ & $.2400000+01$ \\
\hline $.5874000-01$ & $.1000000+01$ & $.2420: 100+01$ \\
\hline $.5626000-01$ & $.1000000+01$ & $.2440000+01$ \\
\hline $.5383000-01$ & $.1000000+01$ & $.2460000+01$ \\
\hline $.5145000-01$ & $.1000000+01$ & $.2480000+01$ \\
\hline $.4913000-01$ & $.1000000+01$ & $.2500000+01$ \\
\hline $.4685000-01$ & $.1000000+01$ & $.2520000+01$ \\
\hline $.4463000-01$ & $1000000+01$ & $.25410000+01$ \\
\hline $.4247000-01$ & $.1000000+01$ & $.2560000+01$ \\
\hline $.4035000-01$ & $.1000000+01$ & $.2580000+01$ \\
\hline $.3329000-01$ & $.1000000+01$ & $.2600000+01$ \\
\hline $.3627000-01$ & $.1000000+01$ & $.2620000+01$ \\
\hline $.3431000-01$ & $.1000000+01$ & $.2040000+01$ \\
\hline $.3240000-01$ & $.1000000+01$ & $.2000000+01$ \\
\hline $.3054000-01$ & $.1000000+01$ & $.2630000+01$ \\
\hline - $2372000-01$ & $.1000000+01$ & $.2700000+01$ \\
\hline $.2696000-01$ & $.1000000+01$ & $.2720000+01$ \\
\hline $.2524000-01$ & $.1000000+01$ & $.2740 i: 00+01$ \\
\hline $.2357000-01$ & $.1000000+01$ & $.27 t 0.00+01$ \\
\hline $.2195000-01$ & $.1000000+01$ & - $2700000+01$ \\
\hline -20) 37000-01 & $.1000000+01$ & $.2800000+01$ \\
\hline $.1884000-01$ & $.1000000+01$ & $.2820000+01$ \\
\hline $.1735000-01$ & $\cdot 1000000+01$ & $.2340000+01$ \\
\hline $.1591000-01$ & $.1000000+01$ & $.2860000+01$ \\
\hline $.1452000-01$ & $.1000000+01$ & $.2880(100)+01$ \\
\hline $.1316000-01$ & $.1000000+01$ & $.2900000+01$ \\
\hline $.1185000-01$ & $.1000000+01$ & $.2920000+01$ \\
\hline $.1059000-01$ & $.1000000+01$ & $.2940000+01$ \\
\hline$. .360000-02$ & $.1000000+01$ & $.2960000+01$ \\
\hline $.3170000-02$ & $.1000000+01$ & $.2900000+01$ \\
\hline $.7330000-02$ & $.1000000+01$ & $.3000000+01$ \\
\hline $.5920000-02$ & $.1000000+01$ & $.3020000+01$ \\
\hline $.4350000-c 2$ & $.1000000+01$ & $.3040000+01$ \\
\hline $.3820000-02$ & $.1000000+01$ & $.30<\cos 00+01$ \\
\hline $.2830000-02$ & $.1000000+01$ & $.3080000+01$ \\
\hline $.1870000-02$ & $.1000000+01$ & $.3100000+01$ \\
\hline $.3500000-03$ & $.1000000+01$ & $.3120000+01$ \\
\hline $.7000000-04$ & $.1000000+01$ & $.3140000+01$ \\
\hline $.7800000-03$ & $.1000000+01$ & $.31600: 00+01$ \\
\hline $.1600000-02$ & $.1000000+01$ & $.31830000+01$ \\
\hline $.2380000-02$ & $.1000000+01$ & $.3200000+01$ \\
\hline $.3130000-02$ & $.1000000+01$ & $.3220000+01$ \\
\hline $.3850000-02$ & $.1000000+01$ & $.3240000+01$ \\
\hline $.4530000-02$ & $.1000 \mathrm{COJ}+01$ & - $3260000+01$ \\
\hline$\because \pm 130060-02$ & $.1000000+01$ & $.3280000+01$ \\
\hline$. .5820000-02$ & $.1000000+0.1$ & $.3300000+01$ \\
\hline . $1,420000-02$ & $.1000000+01$ & $.3320000+01$ \\
\hline . $6380000-0 ?$ & - $1300000+01$ & $.334(18,00+01$ \\
\hline $7530000-02$ & - $1000000+01$ & $.3360000+01$ \\
\hline $.3040000-02$ & $.1000000+01$ & $. .5380000+01$ \\
\hline $.13530000-02$ & $.1000000+01$ & $.34000100+01$ \\
\hline
\end{tabular}




$-.3990000-02$
$-.1430000-02$
$-.9840000-02$
$-.1023000-01$
$-.10590010-01$
$-.1094000-01$
$-.1126000-01$
$-.1155000-01$
$-.1183000-01$
$-.1209000-01$
$-.1233000-01$
$-.1255000-01$
$-.1275000-01$
$-.1293000-01$
$-.1310000-01$
$-.1325000-01$
$-.1338000-01$
$-.1350000-01$
$-.1360000-01$
$-.1369000-01$
$-.1376000-01$
$-.1332000-01$
$-.1387000-01$
$-.1390000-01$
$-.1392000-01$
$-.1393000-01$
$-.1393000-01$
$-.1392000-01$
$-.1339000-01$
$-.1386000-01$

- .3990000-02

- $1000000+01$ - $1000000+01$ - $1000000+01$ - $1010000+01$ - $1000000+01$ - $1000000+01$ - $1000000+01$ - $1000000+01$ - $1000000+01$ - $1000000+01$ $.1000000+01$ - $1000000+01$ - $1000000+01$ $.1000000+01$ $.1000(00+01$ $.1000000+01$ - $1000000+01$ $.1000000+01$ . $1000000+01$ $.1000000+01$ - $1000000+01$ $.1000000+01$ - $1000000+01$ $-1000020+1$ - $1200000+01$ - $10 \operatorname{coc}(1)+1) 1$ - $1030,300+01$ - $1000000+01$ - $1000000+01$ $.100000)+21$
- 34? 0c v0+01 - $344 \mathrm{col} 00+01$ - 3460600+01 . $34801100+01$ - $3500000+01$ - $3520(00+01$ . $3540000+01$ . $3560000+01$ . $3580000+01$ . $3600000+01$ $.3620000+01$ $.3640000+01$ $.3660000+01$ $.3680000+01$ . $3700000+01$ - 37?0000+01

- $3740000+01$

- $3760000+01$

. $3780000+01$

. $3800000+01$

- $3820000+01$

$.3840000+01$

$.3880000+01$

- उEojo out 1

- $3700000+01$

- 392.)0u+01

- 354$)(: 00+01$

- 30100030+01

- 37-31)000+01

- $4000000+01$ 
HP 10 : PCLYNOMIAL APPROXIMATICM PR=Q:EM

2013

. 0000000

- $1960000-n 1$

$.3842000-01$

$.5647000-0.1$

$.7377000-01$

- $9033000-01$

- $1061800+00$

- $1213100+00$

- $1357600+00$

- $1495400+C . C$

- $1626600+00$

- $1751300+10$

- $1969800+c 0$

$.1982200+00$

- $2088700+00$

- $2139300+00$

- $2284200+00$

. $2373700+00$

. $2457700+30$

- $2536600+00$

- $2610.300+00$

- $2679200+00$

- $2743200+i, 1)$

- $2802500+00$

. $2357400+00$

. $2907900+00$

- $2.954100+00$

- $2996100+00$

- $3034200+0 i$

- $3068402+1) 0$

- $3098800+00$

. $312.5600+00$

$.3143000+00$

- $3168900+00$

- $3185600+00$

$.3139100+00$

- $3209600+00$

- 32.17101)+0?

- 3221900+00

$.3223 \div 50+07$

- $3223300+00$

- 3220200+cí

- $3214700+00$

-3\%06900+00

- $3196900+00$

- $3184600+6,0$

- $3170600+(0)$

- $31545 \mathrm{co}+00$

- $3136600+00$

- $3116900+00$

- $3 n 95600+c 0$

. $3072700+00$

- $3048200+c 0$

- $3^{\prime}() 22300+00$

- $2(x) 510 y+40$
- $1000000+01$

- $1000000+01$

- $1000000+01$

- $1000000+01$

- $1000000+01$

- $1000000+01$

- $1000000+01$

- $1000 \mathrm{Ci} 0+01$

- $1000000+01$

- $1300(0)+) 1$

- $1000000+31$

- $100000 j+01$

- $1000000+01$

- $1000000+01$

- $1000000+01$

- $1000000+01$

- $1000000+01$

- 1000 vuvto1

- $1000000+01$

- $1000000+01$

- $1330000+01$

- $1000000+01$

- 100000$)+01$

- $1000000+01$

- $1000000+01$

- $1000000+01$

- $1000000+01$

- $1000000+01$

- $1000000+01$

- $1000000+01$

- $1000000+01$

- $1000000+01$

- $1000000+01$

- $1000000+01$

- $1000000+01$

- $1000000+01$

- $1000000+01$

- 1 :)00000+01

- $1000000+01$

- $1000000+01$

- $1000000+01$

- $1000000+01$

- $1000000+01$

- $1000000+01$

- $1000000+01$

- $1000000+01$

- $1000000+J 1$

- $1000000+01$

- $1030000+01$

- $1000000+01$

- 13$) 0 \mathrm{CJO}+01$

- $1000000+01$

- $1000000+01$

- 1000 isjoto 1

- $1000000+01$
- $0000 \mathrm{Nus}$

. 20001$) 0(1-01$

. $4000000-01$

- $600 \mathrm{r}: 000-01$

- 8c00000-01

- $1000000+00$

- $1200000+00$

- $1430000+00$

- $1600000+00$

- $1830000+00$

- $2000000+00$

- $2200000+00$

- $2400000+00$

- $2600000+00$

- $2800000+00$

- 300u00u+00

. $3200000+00$

- $34 \mathrm{CONOO+00}$

- $3600000+00$

- $3800000+00$

- $4 \cos 0000+00$

- $4200000+00$

$.4401000+00$

- 4 ECO0OO+00

- $4800000+00$

- $50061000+00$

- $5200000+00$

- $5400000+00$

- $5600000+00$

- $5800000+00$

$.6000000+00$

- $0200000+00$

$.6400000+00$

- $6600000+00$

- $6800000+00$

- $7000000+00$

- $7200000+00$

. $7400000+00$

. $7600000+00$

- $780 \pi) 00+00$

- $8000000+00$

- $8200000+00$

- $34100000+00$

- 86croontoo

- 880 cnovtoo

- 9 conotootoo

- $5200000+00$

- $9400000+00$

- $96 \mathrm{C}$ unvotoo

- $800000+00$

- $100(000+31$

- $1020000+01$

$.10410000+01$

- $100.5000+01$

- 1080 i) $00+01$
- 0000000

$.40 \mathrm{~J}$ J0? $)-0.3$

- $1600000-02$

- 3500000-0?

.640Cv0ú-j?

- $1000000-01$

- $144 \mathrm{CDO} 0-01$

- $1560000-01$

- $2560000-01$

$.32400 r 0-31$

$.4000000-)$.

$.4340000-01$

. $576,0000-01$

- $6760000-91$

-7940000-01

- 30000001

- $1024000+30$

- $1156000+03$

- $1296000+0 ?$

- $1444000+00$

- 1 fiucoriotoo

- $1764000+00$

- $1536000+00$

- $2116000+00$

- $2304000+00$

. $2500000+00$

- $2704000+00$

- $2916000+00$

. $3136010+00$

- $33640.00+0$.

- $3000000+00$

$.3 .344000+0$ ?

. $40 ; 6000+00$

$.4356300+00$

. 46:24000+0.)

- $4900000+00$

$.5134000+01$

- $54700010+01$

- $577(0) 0+100$

- E0 B4)00+0?

- $400000+00$

$.6724000+30$

$.7056000+02$

$.7396000+00$

$.7744000+00$

- $8100000+00$

.8464)ก+0

- $3336000+0$.

- $9216000+00$

- $3004000+00$

- $1000000+1$

- $1040400+21$

- $1081600+31$

$.1123600+01$

- 11 búuvto1 
- ?.966.600+00

- $29368 \mathrm{CO}+\mathrm{UO}$

- $2905000+0 \mathrm{C}$

- ?374100+נ0

-? 341100+jo

- $2807200+c 0$

. .277250v+uv

. $2736900+10$

- $2700602+10$

-.2663600+00

- $2626000+00$

. $2587300+00$

$\left..25490 v_{i}\right)+j 0$

- $25098 ; C !+00$

- $2470100+00$

$.2430100+30$

- $238.3700+00$

$.2343 .000+10$

. $3333100+100$

- 2207000+00

- 22.25700+00

$\therefore 1-4.300+00$

- $2142300+00$

- .101200+00

. $053700+20$

- $2018100+00$

- IS TEUOO+OC

- $1935200+10$

$.1893900+00$

- $1852500+00$

- $18116,00+00$

- 1770 \&0n+0c

- $1730100+00$

- $1659700+00$

- $1640000+00$

- $1609630+100$

- $1570200+00$

- $1531100+c 0$

- $1492100+00$

- $1453503+30$

- $1415400+00$

- $1377500+00$

- $1340 \mathrm{c} .00+$.

- $1303200+00$

- $126,6700+50$

- $12306.00+00$

. $11 \cdot 35.300+00$

$.1153300+00$

. $1125000+00$

- $1090<00+30$

- $1057100+00$

- $102330 n+00$

- 1010002-01

- .5 ह800 J-4 1

-)? 71,$) 00-01$

- My53000-01

- se $51000-J$

.3350300-J1
- $1000000+01$

- $1000000+01$

- $1000(0 u+1) 1$

- 1 ग0001

- $1300000+01$

- 1) $02000+01$

- $1000000+01$

- $1030000+01$

- $1000000+01$

- $1000000+01$

- $1000000+01$

- 1 .0000.30+1

- $1000000+31$

- $1.300000+31$

- $1000000+01$

- $1000000+01$

- $1000000+01$

- $1000000+01$

- 1 i) $20000+01$

- 1 vouvuvtc 1

- 1 ) $00000+01$

- $1000000+01$

- $1000000+01$

- $10000 \mathrm{~N} U+01$

- $1020000+1$

- 1 ) Dijouoto 1

- 1$) 00(00)+1$

- 1000 ú + 1

- $1000000+01$

- $1000000+01$

- $1000(0 \mathrm{C})+01$

- $1000000+01$

- $1000000+01$

- $1000000+01$

- $1000000+01$

- $1000000+01$

- $1000000+01$

- $11000000+1$

- $1000000+01$

- 1 1) $00000+01$

- $10000(10+01$

- $1000000+01$

- $1000000+01$

- $1,300000+01$

- $1000000+01$

- $1000000+01$

- $1000000+31$

- $1000000+01$

- $1000000+01$

- $1000 \mathrm{COO} 01$

- 1 ) cuOcioto 1

- $1000000+01$

- $100 \mathrm{CO00+01}$

- $1000000+01$

- $1303003+01$

- $1000003+01$

- $1300 \mathrm{COO}+01$

- 1.) $60000+01$
- 11 ग0ล00+01

- $11201700+01$

$.114000 u+01$

- $1130000+01$

- $11.30000+01$

- $1200000+01$

- $1220000+01$

- $1240000+01$

- $1260000+01$

- $1280000+31$

- $1300030+01$

- $137500+01$

- $1340 \mathrm{i}, 00+01$

- $1360304+01$

- $133.0100+01$

- $1400000+01$

- $1420000+01$

- $1440000+01$

- $140 .(12) i)+01$

- $1480(1) 0+01$

- 152 ก) $30+01$

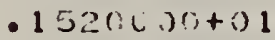

- $1543030+01$

- 15 () $30+u 1$

- $158,00+01$

- 1 ( v) $000+01$

- $10300 j u+01$

- 104 (1) J o + 01

- 1 UE U.juoto 1

- $(631) \mathrm{C}, 01$

- $170 \cup 000+01$

- $17200.30+01$

- $1740000+01$

- $17601000+01$

- $1780000+01$

- $1300(1) 0+01$

- $13200100+01$

- $1340000+01$

- $13 E$ u) $00+01$

- $188 \cdot 3000+01$

- 190() $00+01$

- $1720(0)+01$

- $1940000+01$

- $19 \in 0000+01$

- $1980000+01$

- $2000000+01$

- 20₹UC $00+01$

- 204uv0u+01

. $2060000+01$

- $2080000+01$

- $2100000+01$

- $2120(00+01$

-? $240000+01$

- $21(0)(30+01$

- $2130003+01$

- $2200(00+01$

- $2320000+01$

- $22+0000+31$
- $1210000+01$

- $125440 ?+01$

- $12.951 .00+01$

- $1345.3 i j+j 1$

- $137200+01$

$.1440000+31$

$.1488400+01$

- $1537600+01$

$.1587000+01$

- 1 ij3 4:30+01

- $16 \% 3000+01$

$.17424 .00+31$

- 170 jojocol

- $1345(5) 0+01$

$.1904400+01$

- 15 cio000+01

- ? (1) $1+i(i 0+01$

- $207 \because 600+i 1$

-?131<03+.11

- $2190400+01$

- $2250000+31$

- $2310400+01$

- $23716 . n 0+u 1$

. $2433500+J 1$

- 2496400+01

- $25500(i 0+01$

- 262.4401$)+91$

- $2639000+01$

. $275: x: \cup u+J 1$

-282:400+ 1

- 2. $820000+01$

-235\{4400+01

. $3027600+01$

- $3097600+01$

- $3168400+01$

- 324)nontu1

-3312400+01

- $3355600+31$

- $3455600+01$

- $35744.00+01$

- 3r. 1 l:UCo +01

- $36.30400+01$

- $370.050+01$

- $384160 u+01$

- 39?:400+01

- $430(1000+01$

- $40 ? 30400+01$

$.4161600+01$

- $4243000+01$

- $4326400+01$

. 4410 C J0+31

$.4434400+01$

- $4579600+01$

- $4 E 6.5600+01$

. $475: 2400+31$

$.4840000+1$

. $4: 2: 5400+j 1$

$.5017 \mathrm{i} 0 \mathrm{~J}+31$ 
. $8053000-01$

$.7762000-01$

$.7476000-01$

$.7196000-01$

$.6921000-01$

$.6651000-01$

$.63870010-01$

- $6128000-01$

. 5874000-01

. 5626000-01

. 533 3000-01

$.5145000-01$

$.4913000-01$

$.4535000-01$

$.4463000-01$

$.4247000-01$

.4035000-01

$.3329000-01$

. $3627000-01$

.3431000-01

$.3240000-01$

. 3054000-01

. $2372000-01$

. 2696000-01

. 2524000-01

- $2357000-01$

. $2195000-01$

- 2037000-01

- $1884000-01$

- $1735000-01$

. 1591,000-01

- $1452000-01$

. $1316000-01$

- $1135000-01$

- $1059000-01$

$.9360000-02$

$.8170000-02$

. 7030000-02

$.5920000-02$

-4850000-02

- 3620000-02

- $2330000-02$

- 1 E $70000-02$

. 4500.300-03

$.7000000-04$

- .7000000-03

$-.1600000-02$

- $23330000-0 ?$

- .3130000-c2

$-.3850000-02$

-.4530000-02

-.5190000-02

- .5820000-02

- .6420000-0?

- . $0.980000-02$.

- .7530000-0?

-.8340000-0?

-.3530000-0?
$.1000000+01$

$.1000000+01$

$.1000000+01$

- 1 un $00000+01$

- $1000000+01$

$.1000000+01$

. $1000000+01$

$.1000000+01$

- $1000000+01$

$.1000000+01$

$.1000000+01$

- $1000 \mathrm{co0}+01$

$.1000000+01$

- $1300000+01$

$.1000000+01$

$.1000000+01$

- $1000000+01$

- $10 \mathrm{cocos}+01$

$.1000000+01$

$.100000(0+1) 1$

- $1000000+01$

- $1030000+01$

- $1000000+01$

- $1300000+01$

- $1000000+01$

- $1000000+01$

$.100000)+01$

$.1000000+01$

- $1000000+01$

- $1000000+01$

- $1000000+01$

- $1000000+01$

- $1000000+01$

- $1000 x 0+01$

- $1000 \mathrm{co0}+01$

- $1000000+01$

- $1300000+01$

- $1100000^{\circ} 0+01$

- $1000000+01$

- $10000010+01$

- $1000000+01$

$.1000000+01$

- $1000000+01$

- $1000000+01$

- $1000 v 00+01$

- 1 ) COOC, $0+01$

- $1000000+01$

- 1 uoucouto 1

- 1 : $00000+01$

- $1000000+01$

- $1000000+01$

- 1 uorivootol

- $1000000+01$

- 1 jo ocusoto 1

- $10 \mathrm{cos} 00+01$

$.1000000+01$

- (1) $00000+01$

- 1) $0000 j+01$
$.2260000+01$

- 22 $2313000+01$

. $2300000+01$

$.232(.1100+1) 1$

- $23401000+01$

- $2360000+01$

$.2380000+01$

. $2400000+01$

- $2420000+01$

- $2440000+01$

- $2460000+01$

- $2400000+01$

- 2500r 0u+01

- $2520030+01$

- $254000 u+01$

- $2560000+01$

- $2530000+01$

- 2600 000+01

. $26: 000 u+01$

. $2640000+01$

- 2660uvoto1

$.2680000+01$

. ? $70000 u+01$

$.2720000+01$

. $2740000+01$

. $2760000+01$

. $2780000+01$

- $2300000+01$

- $28 i^{2} 0000+01$

- $2840000+01$

-? $2800000+01$

- 2earur uotol

- $2900000+01$

- 2920000+01

- $2940000+01$

- $29 \in 0 \mathrm{BOO} 00+\mathrm{J}$

- $2980000+01$

- 3000uv0+01

- $302: 000+01$

. $3040000+01$

. $3060000+01$

. 30831$)(100+21$

- $3100000+01$

- 312003001

- $3140000+01$

$.31<0(00+1) 1$

$.3130000+01$

$.3200000+01$

- $3220000+01$

. $3240000+01$

. 320 guootu1

. $3280000+01$

-.330(1iouto 1

$.3320000+01$

$.3340000+01$

- 33 zor o o o o 1

- $33+3 . x(0)+j 1$

- $3400,00 j+01$
$.5107600+01$

$.5198400+01$

$.52330,0+01$

. $53.3: 400+01$

$.5475600+01$

$.55696 .00+01$

$.5664400+01$

$.5760000+01$

$.5856400+01$

. $595.3600+01$

. $6051600+01$

. $6150400+01$

- $6250000+01$

.0330 400+J1

. $6451500+01$

. $6553600+01$

- $6655400+01$

- $6.78 .0000+)$.

- (s) $504400+01$

$.6969000+01$

- $7075000+01$

$.7182400+u 1$

- 7290300+01

$.7393400+01$

$.7507600+01$

$.7617 \mathrm{c} .00+\mathrm{J} 1$

$.7723400+01$

$.7840000+01$

$.7952403+01$

$.8065000+01$

$.8177600+01$

- $8294400+01$

$.8410000+01$

$.8520400+01$

. $8043600+01$

- $761 \in 00+01$

- $8880400+01$

- $9000000+01$

- $9120400+11$

-9241 $000+01$

- $9303600+01$

$.9436400+01$

- $9010000+01$

$.9734400+01$

$.935+600+01$

$.99850010+01$

$.1011243+02$

$.1024030+0$ ?

$.1030840+0$ ?

$.104^{\prime} 3750+0$ ?

- $1002^{2} 750+0 ?$

$.1075843+02$

.10 E.700 Jto?

$.1102243+0 ?$

$.111 \leq 550+0 ?$

$.11285 \cdot 0+0$ ?

$.11424+1+02$

$.1156000+2 ?$ 
$.1000000+01$

$.1000000+21$

$.1000000+01$

- $1200000+01$

- $1000000+01$

$.1000000+01$

- $1000000+01$

. $1000000+01$

$.1000000+01$

$.1000000+01$

$.1000000+01$

$.1000000+01$

$.1000000+01$

- $1000000+01$

- $1000000+01$

$.1000000+01$

$.1000000+01$

- $1000000+01$

$.1000000+01$

- $1000000+01$

$.1000000+01$

- $10000 u j+01$

- $1000000+01$

- 1$) 00000+01$

- $1000000+01$

- $1000000+01$

- $1000000+01$

- $1200000+01$

- $1000000+01$

$.1000000+01$
. $3420000+01$

$.3440000+01$

. $340.0000+01$

$.3430000+01$

$.3500000+01$

. $352.0000+01$

. 354 U $00+01$

$.3560000+01$

. $3580000+01$

$.3600000+01$

$.3620000+01$

. $3640000+01$

$.366,0000+01$

$.3680000+01$

$.3700000+01$

. 37200000+01

$.3740000+01$

. $3760000+01$

. $3780000+01$

- 3800000+01

- $33.0000+01$

- 38401C00+01

- 3800ron+01

- $3680(0) 0+01$

- 3y00unotu1

- 3920000+01

. $3940000+01$

. 39600 $00+01$

. $3580(10)+01$

- $4 \operatorname{cocos} 00+01$
$.1169640+0 ?$

$.118: 360+0 ?$

$.1197100+0=$

$\left..1211^{\prime}\right) 40+.3$

- $1225000+02$

$.1239040+0 ?$

$.1253150+02$

- $1267300+02$

- $1281640+02$

- $129600 n+02$

$.1310440+02$

. $1324960+02$

$.1339560+02$

. $13542.40+02$

- $1360000+02$

$.1383640+02$

- $1393760+0$ ?

$.1413760+02$

$.1428: 340+.32$

$.144400 J+0$ ?

$.1450240+02$

$.1474500+j$ ?

$.1480900+0 \%$

$.1505440+3 \%$

$.1521000+0$ ?

.1536 .64).+0 a$

$.1552350+0$ ?

$.1503160+02$

$.1584 \mathrm{C40+02}$

$.1600000+02$ 
HP 11 : PCLYACHIAL APPROXIMATICN PROELEM

201

- 0000000

- $1: 60000-01$

$.38 .42000-01$

$.5647000-01$

. $7377000-01$

- $9033000-01$

- $1001800+00$

- $1213100+00$

$.1357600+00$

- $1495400+i 0$

- $16,26600+00$

- $1751300+00$

- $1369800+00$

- $1982200+00$

. 20 d $8700+00$

. 2139300+J0

- $22342.00+i 0$

. $2373700+00$

- $2457700+00$

- 253 E $600+00$

- $2610300+00$

- $2679200+00$

- $2743 \ddot{c} 00+0 n$

. $2302600+00$

. 285740$)+00$

. $2907900+00$

- $2954100+40$

- $2906100+00$

- $3034200+00$

- $3068400+00$

- $3098300+06$

- $3125600+00$

. $3149000+00$

- $316.3 .303+00$

- $3185600+J 0$

. $\$ 179100+j 0$

- j20 $3600+00$

- $3217100+00$

- 3221 $300+00$

- $3223700+00$

- $3223300+00$

- 3220200+vo

- 3214700+jo

- 3200racotoo

- $31967 \mathrm{CO}+00$

- $3124500+00$

- $3170600+00$

- $3154500+00$

- $3136600+j 0$

- $3116700+00$

- 30+5000+00

- $3072700+00$

-3048200+00

- jc22.300+0c

- $2 r+5100+00$
- $1000000+01$

- $1000000+01$

- $1000000+01$

- $1000000+01$

- $1000000+01$

- $1000000+01$

- $1000000+01$

- $100000 J+01$

- $1000000+01$

- $1000000+01$

- $1000000+01$

- $1000000+01$

- $1000000+01$

- $1000000+01$

- $1000000+01$

- $15(000)+01$

- $1000000+01$

- $1000000+01$

- $10 \mathrm{cov00}+01$

- $100000_{0}+01$

- $100000 \mathrm{~J}+01$

- $1000000+01$

- 11) $000(10)+01$

- 1 : $00000+01$

- $1300000+01$

- $1000(100+1) 1$

- $1000000+01$

- $1000000+01$

- 1 ) OOO O(.0+01

- $10000011+01$

- $1300000+01$

- $1000000+01$

- $1000000+01$

- $1000000+01$

- 1i) $00004+1$

- $13 \mathrm{co000+01}$

- $1000000+01$

- $1000000+01$

- $10.00 \mathrm{cco+01}$

- $1(100000+01$

- 1100060+01

- 1 uvúl s+01

- $10 \mathrm{c}) 00 \mathrm{~J}+01$

- $1000000+01$

- $1000003+01$

- $1000000+01$

- $1000000+01$

- $1000000+01$

- $1000000+01$

- $1300000+01$

$.10000(0+01$

- $1000000+01$

- 1) 100000+01

- 11) 30$)(10+01$

- 1.) )uvuuto1
.0000003

- $200000-01$

- $4000000-01$

- $5000000-i 1$

- $800 \mathrm{CO00-01}$

- $1000000+00$

- $1200000+00$

- $140000+00$

- $1600000+00$

- $1800000+00$

- $2000000+00$

- $2200000+00$

- $2400000+00$

- $2600000+00$

- $\operatorname{sescocos} 000$

- 300rivootoj

- $3200 \mathrm{co0} 00$

- 3400000+00

- 36 0v600+00

- 36rincion+oo

- $4000000+00$

- $4200000+00$

- 44 into $00+00$

- $46(.0000+00$

- $480 \cup 000+00$

- $5000000+00$

- 520,0000+00

. 540 (.000+00

- $5600000+00$

$.5830000+00$

- $6000000+00$

. $6200000+00$

- $6400000+00$

- $6600000+100$

- $6300<00+00$

- 70ño0otoo

- $7200000+00$

. 74\%6 300+00

- 760 or. $00+00$

- 75uos $00+100$

- acuriootoo

- is?ocivotoo

- $240000+00$

- $360 c 000+00$

- $8300000+00$

- accounotjo

- $9200000+00$

- $940<: 000+00$

- $9600000+00$

- $3300000+00$

- $10(1000 \mathrm{~J}+01$

- $1020000+01$

- $1040(100+01$

- $1000000+01$

- $1030000+01$
- $00000 \mathrm{C}_{1}$

$.4000000-03$

- $1600000-02$

- 3600000-0?

- $6400000-02$

- $1000000-01$

- $1440000-01$

- $1460000-01$

- $2560000-i) !$

- $3240000-01$

- $4000000-01$

$.454 .0000-01$

.57600.00-01

. $6760000-11$

$.7840000-01$

-1) C000!00-91

- $1024000+00$

- $115(00)+01)$

- $1296(00)+20$

$.1444000+011$

- $1600000+00$

- $1-76+0) 0+00$

- $1935000+0$.

- 2116,000+00

- $2304000+0 i$

. $2500000+00$

- ¿70+000+0?

- $2916000+00$

- $3130000+01$

- $3364000+00$

- 36.00000+00

$.3844000+00$

- $4096,0(0+1)$

$.4355000+00$

$.4 .62 .100 \%+00$

- 49000 or ot

- $5184000+00$

- $5476000+00$

$.5775000+0$.

- $603+000+0: 1$

- $6,4031000+0$ ?

- $6724000+01$

- $7056000+00$

$.7376000+00$

- 7744001)+(0i)

- $81000(00+01$ )

- $346400 u+00$

- 883600u+00

- D216000+0)

- $\rightarrow \in 04 C 00+00$

- $1000000+c !$

- $1040400+01$

- $1081600+01$

- $11 ? 3600+01$

- 11 - $4(0)+01$
$.0000) 0 ?$

- $1000000-0<$.

- $6000000-04$

- $2200000-03$

$.51(1000)-7,3$

- $1000000-02$

$.1730000-02$

- :740jo-jo

-4100000-Uz

- ja $30000-j$ ?

- 3uovir o-b?

- $1065300-01$

$.1382000-01$

- 175800.01

- $2165000-1$

- $? 70$ )

- $3277030-01$

- 3030)00-01

- 456filno-01

- 54 E $7(: 0.0-1) 1$

- 6400$) 00-01$

- 7409000-i) 1

. $3513060-01$

- $9734000-01$

- $1105000+i 0$

- $12500(10+1) 0$

- $1406 \cdot 00+c 0$

- $1574600+00$

- $1756 ? \mathrm{C}+10$

- $1951100+.0$

- $2150000+0=$

- $2383300+00$

- $252140 j+1) 5$

- ?त? $5000+00$

$.3144300+00$

. 3430)00+ก0

- $3733500+00$

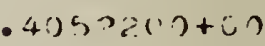

. $4339370+10$

-47it55:1J+30

- 11 a $0 n: n+(\vdots)$

- 55:137iv +o

- $540700(1+1)$ ?

- E.36 vi $10+u c$

- $531+790+00$

- $72 \div 3000+10$

$.779 . ; 000+00$

- $3305600+01$

- ध8 $347400+00$

$.9411300+10$

- $1300000+01$

- $1061>10+01$

- $112+50+01$

- $11 \geqslant 1,320+31$

- 1 ?:-710+u1 
- 2506úcotuu - $2936800+00$ . $2906000+00$ - $2874100+i) C$ - $2341100+00$ - $2307200+00$ - $2772500+00$ . $2736900+00$ $.2700600+00$ - $2663600+00$ . $2626000+00$ . $2587800+00$ . $2549000+00$ $.2509 j 00+0 u$ - $2470100+00$ . $2430100+i) 0$ . $2389700+00$ . $2349000+00$ $.2308100+00$ . $2207000+00$ . $2225700+00$ - .1184300+00 - $\therefore 142300+0 C$ . $2101200+00$ - $2059700+00$ - 2013100+00 - $197 t 600+J 0$ - $1935200+00$ . $18938 \mathrm{coto0}$ - $1852600+00$ - $1811500+00$ $.1770800+00$ $.1730100+00$ $.1689700+00$ $.1649600+00$ $.1609800+00$ $.1570200+00$ - $153100 n+j 0$ - $149 ? 100+00$ $.1453500+00$ $.1415400+00$ $.1377600+00$ . $1340200+00$ - $1303200+J 0$ - $1266700+00$ - $1230600+01$ $.1195000+00$ . $1159800+00$ $.1125000+00$ $.1090800+00$ $.1057100+00$ - $1023800+00$ - 3) $10000-01$ -5 53000-01 . 127100.)-01 $.8958000-01$ . $3651000-01$ . $3350000-01$
- $1300000+01$ - $1000000+01$ - $1000000+01$ - $1000000+01$ - $1000000+01$ - $1300000+01$ $.1000000+01$ $.1000000+01$ $.1000000+01$ $.1000000+01$ - $1000000+01$ - $1000000+01$ - $1000000+01$ - $10 u v u(j)+i$ - $1000630+1$ - $100<v 00+01$ - $1000000+01$ - $1000000+01$ - $1000030+01$ - $1000000+01$ - $1000000+01$ - $1000000+01$ - $1000000+01$ $.10000 .00+01$ - $1000000+01$ - $1000000+01$ - $1000000+01$ - $1300000+01$ - $1000000+01$ - $1000000+01$ - $1000000+01$ - $1000000+01$ - $1000000+01$ - $1000000+01$ - $1000000+01$ - $1000000+01$ - $1000000+01$ - $1000000+01$ - $100000 J+01$ - $1000000+01$ - $1000000+01$ $.1000000+01$ - $1000000+01$ - $1.200000+01$ - $1000000+01$ - $1003000+31$ - $1000000+01$ - $1000000+01$ - $1000000+01$ - $1000000+01$ - $1000000+01$ - $1000000+01$ - $1000000+01$ - $1000000+01$ - $1000000+01$ - $1000000+01$ - $1003000+01$ - $1000000+01$
$.1100000+01$ - $1120000+01$

- $1140000+01$

- $1160000+01$

- $1100000+01$

$.1200000+01$

- $1220000+01$

- $1240000+01$

- $1260000+01$

- $1280000+01$

- $1300000+01$

$.1320000+01$

- $1340000+01$

- i3usjucoto 1

- $13313000+01$

- $14011000+01$

$.1420000+01$

$.1440000+01$

$.146 .0000+01$

$.1430[0+01$

- $150(1000+01$

- $1520000+01$

. $1540000+01$

- $1500 \mathrm{~s} 00+01$

- 1 ว

$.1600000+01$

- $1620000+01$

$.1640000+01$

- $1660000+01$

- $1680000+01$

- $1700000+01$

- $1720000+01$

$.1742000+01$

- $1760000+01$

$.1780000+01$

- $1800000+01$

- $1820000+01$

- $1840000+01$

- $1860000+01$

$.1880000+01$

- $1900000+01$

- $1920000+01$

- $1440000+01$

- $1960000+01$

- $19150000+01$

. $2000000+01$

- $2020000+01$

$.2040000+01$

. $2060000+01$

- $2080000+01$

. $2100000+01$

. $2120000+01$

$.2140030+31$

$.2100000+01$

- $2130020+01$

- $200000+01$

- $2220000+01$

- $224000 \mathrm{C}+01$
$.1210000+01$

$.1254400+01$

- 12 c0500+01

$.1345600+01$

. $1352400+01$

$.1440000+01$

$.1483400+01$

$.1537600+01$

$.1587600+01$

$.1633400+01$

$.1690000+01$

$.1742400+01$

$.1735600+1$

$.1849600+j 1$

$.1304400+01$

- $1980000+21$

- $2016400+01$

- $2.073600+01$

$.2131600+01$

$.2130400+01$

$.2250000+01$

- $2310400+01$

$.2371000+01$

- $2433800+01$

$.24564 j 0+01$

$.2550000+.11$

. $2624400+01$

. $2689600+01$

$.2755600+01$

. $282 ? 400+01$

$.2830007+01$

. $2958400+01$

. $3227500+01$

$.3097600+01$

$.3168400+01$

$.3240000+01$

$.3312400+01$

$.3335600+01$

. $3453600+01$

$.3534400+01$

$.3610000+01$

. $368640 \mathrm{~J}+01$

$.37636,00+01$

- $3841600+01$

$.3920400+01$

$.4001000+01$

$.40804(0+01$

. $4161600+01$

$.4243600+01$

$.4326,400+01$

$.4410000+01$

$.449440 n+01$

. $4579600+01$

$.40 .65600+01$

$.475 a 400+01$

$.4840000+01$

$.4923400+0$ ?

$.5017600+01$

. $1331000+01$

$.1404330+31$

$.1481540+01$

- $1500300+21$

$.1 \leq 43030+01$

$.1728700+01$

$.1415350+1$

- 1 coth?o+o 1

- $2000330+31$

$.2057150+01$

. $2197000+1$

- $2390 \times 70+01$

-? $\begin{aligned} & 0 \\ & \text { - }\end{aligned}$

- $261 \div: 0+51$

. $262-317)+1$

. $744300+01$

- $2853 \geq 00+11$

- $2335,330+02$

$.311 \geq 140+01$

$.3241790+01$

. $3375000+01$

- $3511310+01$

. $36 \leq 2250+01$

. $37+6400+) 1$

. $3444310+01$

- $4035000+01$

. $7251530+01$

$.4410940+21$

$.4574300+01$

$.4741 \in 30+01$

$.4913000+01$

$.5043450+21$

. $5263020+01$

$.5451730+01$

- $5635,750+1$

. $58332000+01$

$.6028570+1) 1$

. $6239300+01$

$.6434860+01$

- $\epsilon<44570+01$

- 685900001

- 7077:3:00+01

$.7301330+01$

$.75295+0+01$

$.776 .259)+(01$

- $8000300+01$

$.8242410+01$

$.84896 .60+31$

$.8741520+01$

- 3998910+01

- $9261000+01$

$.9523130+1$

- $9800340+21$

- $1007770+02$

$.1036323+32$

- $1004300+02$

- $1044105+$ )?

$.112374 ?+1) ?$ 
$.3053000-01$

$.7702000-31$

$.7475000-01$

$.7130000-01$

$.6921000-01$

. 105100101

. $1337000-01$

. ti $128000-01$

. $5874000-01$

$.5626000-01$

$.5383000-01$

$.5145000-01$

$.4913000-01$

. 46:35000-01

$.446,3000-01$

$.4247000-01$

$.4035000-01$

. $3329000-01$

. 3627000-01

$.3431(10)-01$

. $3240000-01$

$.3054000-01$

- $2372000-01$

$.2696001-01$

$.2524000-01$

- $2357000-01$

- $2135000-101$

. $2037000-01$

- $1834300-01$

$.1735) 00-01$

- 15:10C0-01

$.1452000-01$

$.1316000-01$

$.1135000-01$

$.1059000-01$

. $9360000-02$

$.8170000-0$ ?

- 7030000-0?

- 5920000-02

$.425001) 3-122$

- 3:3\%0000-0?

$.2330000-02$

$.1870000-1) 2$

. $.5500000-13$

.70 jouno-u4

-.7800000-0.3

-.1Ev000J-02

- . $323000 j-02$

-.3130000-02.

-. $3350000-02$

-.4530000-02.

- $\therefore 190000-02$

- . .3?000.1-0?

- $\therefore 420000-112$

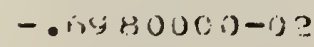

-.753000 : 0-02

-.83040000-0\%

- .530000-6?
$.1000000+01$

- $1000000+21$

- $1000000+01$

- $1,100000+i 21$

- $1003000+01$

- $1000000+01$

- $10000 \% 0+01$

$.1000000+01$

$.1000000+01$

- $1000000+J 1$

- $1000000+01$

- $1000000+01$

- $1000000+01$

- $1000000+01$

- $1000000+21$

- $1000000+01$

- $1000000+01$

- $1300000+01$

- $1000000+01$

$.1000000+01$

- $1000000+01$

$.1000000+01$

- $1000000+01$

- $13000 n j+01$

- $1000000+01$

- $1000000+01$

- $1300000+01$

- $1000000+01$

- 1$) \cos 30+01$

- $1000000+01$

- 1 junconto1

- $100000 u+01$

- $1030000+01$

- $1000000+01$

- $1000000+01$

- $1030000+01$

$.12000(1+01$

$.1000(100+01$

- $10300,3 j+01$

- 10 coscotol

- 1 vooverotul

- $1000000+01$

- $1000000+1) 1$

- $1000000+01$

- $1000000+01$

- $1000000+01$

- $1000000+01$

- $1.200000+01$

- $1000000+01$

- $100000 u+01$

- $10 \operatorname{coc} 00+01$

- $100000 u+01$

- $1300000+01$

- $1000000+01$

- 1 1) $00003+01$

- $1100000+01$

- 1 1 co00: $2+11$

- 1 c) con. $3+01$
- $235(0) 00+01$

- $22 \sin 0000+01$

- $2300000+01$

- $23200.00+01$

. $234:(x) 0+01$

- $236(\cdot 6.000+01$

- $2380000+01$

- $2400000+01$

$.2420000+01$

. $2440000+01$

$.2460000+01$

$.24800,00+01$

- $250(1000+21$

- $2530000+01$

- $2540 \mathrm{ar} 30+01$

- $2500(0)+01$

. $25(3.1) 10+01$

- $2600000+01$

- $2620000+01$

- ?ranuDot 1

- $2600000+01$

- $263(0,1,00+01$

- 2700(incisto

- $272 x-00+01$

. $2740000+01$

- $27 \% 10(2) 00+01$

- 2781$)(100+01$

- $28(i) \cup 00+01$

- $282(1000+01$

- 2840000+01

- ?860000+01

- $2880000+01$

- $2300000+01$

- $2920000+01$

- $2740 n 00+01$

- $2960000+01$

$.2930000+01$

$.3000000 \times 01$

$.3020000+01$

$.304+1000+01$

$.3060000+31$

- 3036) $100+01$

- 31 vurioutor

- $3120000+01$

. $3140000+01$

- $3160000+01$

$.315(1) 00+01$

- 3208100+01

- 3220000+01

$.3240000+01$

$.32601700+01$

. $3280000+01$

$.3300000+01$

- 33.20000+01

.33401:v0+01

. $3350000+01$

$.33308 j 0+1) 1$

. $34(8000)+1$
$.5107000+01$

$.5102400+01$

$.5230000+1$

$.53(23400+i) 1$

$.5475000+01$

. $5560600+1) 1$

- $56(1+400+) 1$

$.576,0000+(01$

$.585(+1) 0+01$

. $5953600+01$

$.6051600+01$

. $6150400+01$

- $6250000+21$

- $635(1400+01$

. $64512 ? 0+11$

. $6553500+(11$

. $6.650400+31$

$.6760000+01$

- $0364400+01$

- $690(0), 0.2+01$

. $707560 u+01$

. $71832400+01$

$.7230000+01$

$.7393400+01$

$.7507000+J 1$

. $7617.500+11$

$.77283400+01$

$.7840000+01$

- $\$ 9524(10+01$

- $4065600+1) 1$

- $817960 n+01$

. $52 \div 4400+01$

- $8410000+01$

- $8525400+01$

- $8043600+01$

. $376.1600+(1) 1$

- $8880400+01$

- $9000000+01$

$.9120400+01$

- $9241500+01$

- $93(3000+01$

$.048(400)+31$

- 3010000+01

- $9734400+01$

$.9359000+01$

- $9985000+01$

$.1011240+6 c^{2}$

- $1034000+02$

$.1036840+02$

- $1 \mathrm{C} 44750+0$ ?

. $1062750+0$ ?

$.1075840+02$

- $1089000+1) 2$

. $1102 ? 40+0$ ?

$.1115550+0$.

$.1123: 00+02$

$.1143440+0$ ?

- $115.000+0$.

$.11+4313+j$,

$.1135335+7 ?$

- $1216700+1) ?$

- $124: 3>17+3$ ?

$.1281301+3$

-1?144:?6+5?

. $13+8117+v ?$

$.1332400+0 ?$

$.1417 ? 49+12$

$.1452373+1$ ?

$.14833(0.34+12$

- $152003 \%+3 ?$

- $15,5,2,01+32$

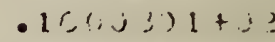

- 1< $367 r+10$

- 1 c) $17132 x+3$ ?

- $17172=1+12$

- $17 \div 7,101)+1) 2$

$.17-4473+0$ ?

- Is 30.174ti?

- 1 riszl10+is?

$.1924: 13+02$

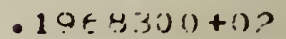

. ?012345+1?

. $005734 ?+1) ?$

$.210245 \lambda+0 ?$

$.2148435+0 ?$

. $2195200+\cdots ?$

$.2242 .577+0 ?$

- $2250631+12$

- $233036.6+1$ ?

. $2383747+98$

$.2439100+1 ?$

$.24<97(y+i)$ ?

- $2541 \therefore 11+3$

- $259.3434+j 2$

- $264+3.37+1 ?$

- 270$) 00 j+0 ?$

- 27 i4 $31+0 ?$

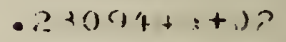

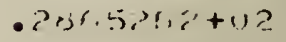

- $2+\geq 131+1+$

- $24731(10+1)$ ?

$.3037178+1$

. 304:3 $414+02$

. 31 ictisoto?

- 3a. 157 í $3+0$ ?

. 3?70 $327+18$

- $33333055+02$

$.3401322+02$

$.3454593+) 2$

- $352875,5+02$

- $350.3700+02$

- $3655427+0 ?$

- $37 ? 5(\cdot 7)+.32$

- $37-3.31 u+02$

- $3 \%, 1+i+7+1=2$

-4:? 
- . 3990000-02

- . $433000-0 ?$

-.9840000-02

-.1023000-01

- 1059 c0 O-01

-.1094000-01

-.1126000-01

-.1155000-01

-.1183000-01

-.1209000-01

-.1233000-01

-.1255000-01

-.1275000-01

-.125 3000-01

-.1310000-01

-.1325n00-01

-.1333000-01

-.1350000-01

-.1360000-01

-. $136,9000-01$

-.1376000-01

-. $1382000-01$

-.1337000-01

- $1390000-01$

- $1302000-01$

-.13i3000-01

-.1393000-01

-.1392000-01

-.1389000-01

-.1386000-01

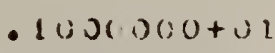

- 1. cJ00J+u 1

- $1000000+31$

- $11000000+01$

- $1000000+01$

- $1000000+01$

- $1000000+01$

- $1000000+01$

- $1000000+01$

- $1000000+01$

- $1000000+01$

- $1000000+01$

- $1000000+01$

- $1000000+01$

- $1000000+01$

- $1000000+01$

- $1000000+01$

- $1000000+01$

- $1000000+01$

- $1000000+01$

- $1000000+01$

- 1300 (1)

- $1000000+01$

- $1000000+01$

- $1000000+1$

- $1000000+1$

- $1000000+01$

- $1000000+01$

- $1000000+1$

- $1000000+01$
. $3420(2:)+01$

$.34+2) \cdots+01$

- 3460i00+01

- $3480000+01$

- $3500000+01$

. $3520000+01$

- $3540000+01$

. $3560000+01$

- $3580000+01$

. $3600000+01$

. $3620000+01$

. $3640000+01$

- $3660000+01$

. $3680000+01$

- $3700000+01$

- $3720000+01$

- $37406,00+01$

- 376 cioutol

$.373(i v 0 u+01$

$.3800000+01$

- $382 C(000+01$

- $3.340 ? 31+01$

- $3800000+01$

- $3 \sin 0(100+21$

- 3300000+01

- 392.)i00+01

-3940u00+01

- 3sticuooto1

- 34:30100+01

. $4000000+01$
-116 残 $10+n ?$ - $1133 . ., 3+i$.

- $1157160+32$

- $1211040+0$ ?

- $1225000+02$

- $1239040+02$

- $1253160+02$

- $1267360+02$

. $1281640+02$

- $1296000+02$

$.1310440+02$

$.1324960+02$

- $1339550+02$

. $1354240+02$

- $1369000+02$

$.1363840+02$

. $13 \rightarrow 8760+02$

$.1413700+02$

$.1428840+02$

$.1444000+0$ ?

$\left..1459^{\prime} c^{\prime} 41\right)+102$

$.1474560+0 ?$

$.14 .39360+0 ?$

$.1505440+03$

. $1521000+02$

$.1538+40+02$

$.155236,0+02$

- $1568160+0$ ?

$.1534040+02$

- IE CJojotu?
- + (1) :111< $6+\therefore$

- 41) ? $7.9+1$

- $4142174+22$

. $4214419+02$

$.4287500+02$.

$.4301421+02$

$.4436186+0$ ?

$.4511802+02$

$.4588271+02$

$.4665600+02$

$.474370 .3+02$.

$.4822354+02$

$.4902790+1) 2$

$.4943603+0 ?$

- 50 E $5300+02$

$.51+7835+0 ?$

. $5231362+u ?$

$.5315733+10 ?$

$.5401015+02$

. 543?2200+0?

$.5574207+0$ ?

- Th⿱一𫝀口) 31$)+02$

. $575121 \%+u ?$

. $594110^{\top}+0$ ?

$.5031000+02$

. $61123625+02$

$.6116299+0 ?$

$.6209914+0$ ?

$.6304479+10$ ?

$.0400030+02$ 
HN12: PCLYNCMIAL APPRCXI AATIGN PROBLEI

201

.0000000

- $) 000000$

- 136,0000-01

- 0000000

$.3842000-01$

.0000000

- 56.47000-01

$.1000000-04$

$.7377000-01$

$.4000000-04$

$.9033000-01$

- $1000000-0.3$

$.1061800+00$

-2100000-03

- $1 ? 1.3100+00$

- $3800000-03$

- $1357600+00$

- c $60000 u-c 3$

- $14 \subseteq 5400+00$

- $1050001-0 ?$

- $1 F 26000+c 0$

- $1600000-0 ?$

- $1751.300+00$

- $2340000-02$

- $1364400+00$

- 3320 i)०-02

- $1982200+00$

- $4570000-02$

- $2088700+00$

- $6150000-02$

- $2183300+00$

- $8100000-02$

- $2284200+00$

- $1049000-01$

. $2373700+00$

- $1336000-01$

. $2457700+00$

- $168 \mathrm{cCO} 0-01$

- 2536 tioj+o

- $2085000-01$

- $2610.300+00$

. 2560000-01

. $2679200+00$

- $3112000-01$

- $2743200+00$

- 3748000-01

. $28026 U 0+00$

. 4477J00-01

$.2857400+00$

- 5301300 0-01

$.291779(1) 0+(1)$

- :3:50j0)-01

. $2954100+00$

- $731 ? 0010-01$

. $310100+00$
$.1000000+01$

$.1000000+01$

$.1000000+01$

$.1000000+01$

$.1000000+01$

$.1003000+01$

$.10000 \mathrm{cot} 01$

- 1)00000+01

- $1000000+01$

$.1,00000+11$

$.1000000+01$

- $100(: 000+01$

- $1000000+01$

- $1000000+01$

$.1000000+01$

$.1000000+01$

$.1000000+01$

$.1000063+01$

$.1000000+01$

- $1000000+01$

$.1000000+01$

$.1000000+01$

$.1000000+01$

$.1000000+01$

$.1000000+01$

$.1000000+01$

$.1000000+01$

$.1000000+01$
.0000 .100

$.2000000-01$

$.4000000-01$

$.6000000-01$

$.8000000-01$

$.1000300+00$

$.1200000+00$

$.1400000+00$

- $160(1) 00(1+00$

$.1800000+00$

$.2000000+00$

.$<2(30) 00+00$

$.2400000+00$

$.2000000+00$

$.2900000+00$

$.3000 j 00+00$

$.3200000+00$

$.3400000+00$

$.3600000+00$

$.33 .00000+00$

$.4000000+00$

$.4200000+00$

$.4400000+00$

$.4600000+00$

$.4800000+00$

$.5001) 000+00$

- $52010000+00$

$.540 u(i v)+00$
.00000 .10

.0100000?

$.400003-03$

$.1000000-24$

$.1600000-02$

$.60000 .20-04$

$.3600000-0 ?$

$.2200000-03$

$.6400000-02$

$.512 .20(2-) 3$

$.1020000-01$

- $1006: 9(1)-1) ?$

$.1440000-01$

- $17390(0-1) ?$

$.1900000-01$

$. ? 7401100-3 ?$

- $25 x(01)-11$

$.41 \cdot 0000-3 ?$

$.3240000-01$

- is $10000-1 ?$ ?

$.4000000-01$

- $3.3230000-02$

$.484 \cup 000-01$

- $1065000-01$

$.5760000-01$

- $1 \geq 32000-01$

$.8700000-01$

- $175300 n-01$

- †े440000-01

$.2195000-01$

.9: $100000-01$

. $27: 1000(0-1) 1$

- $1024000+00$

$.3 .277 .0 .0-31$

$.1155000+00$

- $39.30100-01$

$.129(000+(i)$

$.405(3,5) !)-21$

$.1444000+00$

.543711001

$.1000000+00$

$.8470000-01$

$.1764000+00$

- 7100000-0i

$.1936000+00$

- $5518000-01$

- $2116000+00$

$.9734 \cap(10-01$

$.2304000+00$

$.1105000+00$

$.2500000+00$

$.12500(10+00$

$.27(1) 40(1)+1)$

$.1406100+00$

. $2.916000+00$
$.1574 .110+00$ 
. $0503000-01$

. $3034200+00$

- $9834000-01$

. $3069400+00$

$.1131600+00$

. $3098000+00$

- $1236000+00$

- $3125600+00$

- $1477600+00$

- $3149000+00$

- $1677700+00$

$.3168700+00$

- $13975 \mathrm{CO}+0 \mathrm{~J}$

- $3135600+J 0$

- ? 13810()$+00$

- $3199100+00$

. 2401000+00

- $3209600+00$

- $2687400+0 J$

. $3217100+00$

- $2998700+00$

- $3221900+00$

- $3336200+0$ :

$.3223900+00$

. $3701500+00$

- $3223100+0 . j$

. $4034000+00$

$.32202 \mathrm{CO}+\mathrm{ju}$

. $4521200+00$

- $3214700+00$

. $4978700+00$

$.32069 \mathrm{C})+0 \mathrm{j}$

$.5470100+u u$

. $3146900+00$

- 5997000+00

$.3184300+00$

. $0561000+00$

- $3170600+00$

$.716 .3500+00$

$.3154 .500+00$

. $7807500+00$

- $3136.600+00$

- $34 ! 3500+00$

-.3116:00+100

. 12237co+0

- 305 5ucotoc

- 1 1) $00000+01$

$.3072700+00$

- $1032430+01$

- 30 48:00+00

- $11(0,9) 61)+01$

$.3022300+00$

- $126 ? 480+01$

- $\therefore 510 u+00$

- $13 E 0490+01$

- sstet00+00

- 1 it $4100+01$

-? $710300+00$
$.1000000+01$

$.56000010+00$

$.3130000+00$

- $1740200+00$

$.1000000+01$

$.5320000+00$

$.336(20) 2+(2)$

$.1351100+c 0$

$.1000000+01$

$.6000000+30$

- $1000000+01$

$.6200000+00$

$.1000000+01$

$.6400000+00$

- $1000000+01$

$.1000 c 0 u+01$

$.100000+01$

$.1000000+01$

- 1000000+01

$.1000000+01$

$.1000000+01$

$.100001: 3+01$

- $1000003+01$

-11300000+01

$.1000000+01$

$.1000000+01$

$.1000000+01$

$.1000003+01$

- $1000000+01$

$.1000000+01$

$.1000000+01$

$.100060 u+01$

$.1000000+01$

$-130(00)+01$

$.1000000+01$

$-1 \cup 1) \cup(00+1) 1$

$.1003000+01$

$.10000110+01$
$.6600000+00$

- $6800000+00$

$.7000000+00$

$.7200000+00$

$.7400000+00$

$.7000000+00$

$.7800 .700+00$

- 30เก)0u+00

- $8200000+00$

$.9400000+00$

$.3600000+00$

- E. $300000+00$

- conoconotoo

$.4200000+00$

$.9400000+00$

- $5000000+00$

$.7900(100+00$

$.1000000+01$

$.1020000+01$

$.1040000+01$

$.1060000+01$

- $1030(0)+i) 1$

$.1100(00+01$

$.4356000+00$

$.4624000+00$

$.4500000+3$

$.518400(1+0)$

$.5: 760 \div 0+013$

$.577 \dot{U} \cup(0+0$.

$.60+4.0(0+30$

$.6400(100+0)$

$.6724000+00$

$.7050000+C 0$

$.739000+0)$

$.7744000+00$

$.83100030+0.3$

$.8404) 30+0 i$

$.8835000+30$

$.9210000+00$

$.9604000+1)$

$.1000000+01$

$.1041400+01$

- $10.31600+01$

$.1123600+01$

$.11+0400+(01$

$.1210000+01$

$.1120100+u 1$

$.12544100+(11$
$.3000000+00$

$.3244000+00$

$.4096000+00$

$.2160000+100$

$.2323300+00$

$.2621400+00$

- $2375700+00$

- $3450000+130$

$.3732500+100$

$.4) 5 ? 200+30$

$.4 .3 \div ; 3.2 n+00$

$.47455 .10+130$

$.12120(100+00$

$.5513700+00$

- $59 ? 7000+01$

$., 3609: 0+00$

(i) $1147100+10$

- $7 ; 00000+00$

$.7735 i(3)+01$

$.9305800+(2)$

$.8847+00+10$

- $9411500+00$

$.1000000+01$

$.10 ? 1: 10+01$

- $11 \because 4.3 i 0+)$.

- $11913: 2+01$

- $125471+1+1$

- $1331006+i 1$

- $1+13+2,1+31$
- $3144500+20$ 
$.1573520+01$ - $2906000+00$ - $16.38960+01$ $.2874100+00$ - $1810640+01$ - ?841100+00 - $1933780+01$ - $2307200+00$ - $2073000+01$ . $2772500+00$ - $2215330+01$ - $2736900+00$ - $2.364210+01$ . $2700600+00$ .252 .047 ) +01 - $2663600+00$ - $2634.350+01$ - $2026000+00$ - $2856100+01$ . $2587800+00$ . $3035900+01$ - $2549000+00$ - $3224180+01$ - ? $509870+00$ - $3421020+01$ . $2470100+00$ . $3626740+01$ - $430100+00$ - $3341600+01$ - $2389700+00$ - $4065870+01$ . $2349000+00$ - $4249520+01$ - $2308100+00$ $.4543720+01$ - $2267000+00$ $.4797850+01$ - ¿225700+00 - $5062500+01$ - $184300+00$ - $5337950+01$ .? $1423 \mathrm{CO}+00$ - $5624490+C_{1}$ - $2101200+00$ . $5922410+01$ . $2059700+00$ - $3232010+01$ - $2018100+00$ . $6553600+01$ . $1976000+00$ - $0837480+01$ - $15.35200+00$ - $72330350+01$ $.183 .3300+50$ - ?54 $33.30+01$ - $185 \% 00+00$ . >9E5540+C 1 - $13116,00+0.0$

$$
.1000000+01
$$$$
.1140000+01
$$$$
\text { - } 1267500+01
$$$$
.116 .0000+01
$$$$
\cdot 1.34,5,00+(11
$$$$
.1130000+01
$$$$
.1362420+01
$$$$
.1200000+01
$$

$.1000000+01$

$.1000 \mathrm{CCO}+01$

$.1000000+01$

$.1000000+01$

$.1000000+01$

$.1000000+01$

- $1.200000+01$

$.1000000+01$

$.1000000+01$

$.1000000+01$

$.1000000+01$

$.1000000+01$

$.1000000+01$

$.1000000+01$

$.1000000+01$

$.1000000+01$

$.1000000+01$

$.1000000+01$

$.1000000+01$

$.1000000+01$

- $1000000+01$

$.1000000+01$

$.1000000+01$

- ia 30 uro+ol

$.10000(1)+01$

$.10000(10+01$
$.1220000+01$

$.1240000+01$

- $1260000+01$

$.1280000+01$

$.130000 u+01$

- 1 3? $? 0000+01$

$.1340: 130+11$

$.1350 .00+21$

$.13+30000+01$

$.1400000+01$

$.1420000+01$

$.1440000+01$

$.1400100+01$

- $1480000+01$

- $1506,00 u+01$

- 1520000+01

- $15400 \cup 0+01$

- $1560000+01$

- $15830100+01$

$.1600000+01$

$.1620000+01$

$.1640000+01$

$.1600000+01$

$.16310100+01$

$.1700000+01$
$.1440000+01$

$.1488400+01$

. 15ख7600+01

$.1587+0,0+01$

$.16333400+01$

$.1630000+01$

$.1742400+01$

$.1795600+01$

$.1345600+01$

- $14.14400+01$

$.1960000+01$

$.201 \epsilon 400+01$

$.2075600+01$

$.21316 \cot 31$

$.2190400+01$

$.2250000+01$

$.2310400+01$

$.2371600+01$

$.24 .3 \pm 000+01$

$.2496 .400+01$

-2560000+01

$.26 .24400+01$

- $2.6,8+200+01$

$.275500 u+01$

$-2822+00+01$

$.2300000+01$
. $1451540+i) 1$

$.1560400+01$

$.164 .353 .2+01$

$.17 \because 3000+01$

$.1815(350+01$

- 130t:

- oor o $340+11$

. $20771: 0+01$

. $: 1971300+01$

$.2299 \cdot 77)+01$

$. ? 4061(1)+01$

$.2515460+01$

- 21:283070 +41

$.2744(00+1) 1$

$. .286,1290+01$

$.2985780+i 1$

- $3112147+1) 1$

$.32 .41737+01$

$.33750(0+31$

$.3311 \cdot 310+01$

- . $3 t \cdot 2: 30+01$ ,

$.37564 ? 0+01$

. $2544.310+111$

$.4045000+01$

$.4251530+01$

$.4410: 40+: 1$

$+4 ? 4,00+1$

$.4741630+31$

$.4613 \cdot 1 \%+11$ 
- $1000000+01$

$.1720000+11$

$.2454400+01$

- $1740 i v 0+01$

- $170(2,00+01$

- 1ง0U⿺尢丶01

.1780 or: $00+01$

- $1800 u \dot{u} 0+01$

$.10000(0+01$

- $1320000+01$

- $1000000+01$

$.1840(, .30+01$

$.1000001+01$

- $13+.(100+) 1$

$.1000000+31$

- $1830000+01$

- $101100(0+01$

$-1+1) 66,16+01$

- $1200600+u 1$

. 1 , $\therefore(100+01$

-1) 1)C(1)+01

- $10 \cos (5)+01$

- $130.3200+00$ - $1475785+02$ - $126,6701+36$ - $1536954+62$ - $1230600+00$ - $1600000+02$ - $1175000+00$ - $1 \epsilon 54 \cdot \exists \epsilon \epsilon+02$ . $115 \% 300+j 0$ - $1731391+0 ?$ . $1125000+00$ - $13 i) 0 \Omega 14+0 ?$ - $1000500+00$ . $1871774+12$ - $1057100+00$ . $1944310+02$ - $102.3800+00$ $.2019963+02$ - (9911)000-01 - 20 $97274+0 ?$ - 538000-01 - $2176782+0 ?$ - $9271000-01$ . $225.3531+.2$ - 045 $3000-01$ - $2342560+02$ - (36) $51000-01$ . $2428913+J 2$ - $3350000-C_{1}$

. $2517631+12$ -. $053000-01$ . $2508759+02$ -77रि? ग0-01
- $1300(20) 1$

$.1000000+01$

$.10 \mathrm{c}(1)(i)+01$

.1\%06:000+01

- $1300000+31$

$.1000000+01$

$.1000000+01$

$.1000000+01$

$.1000000+01$

$.10001000+01$

$.1000000+01$

$.1000040+01$

$.1000000+01$

-1) )

-224.)1 $11+01$

$.1000000+01$

- 2200$)(00+01$

$.12000110+01$

$.193(1) 00+01$

$.2000(\cdot 9) 0+01$

- $202(91) 1) 0+(11$

$.2040(1) 0+01$

$.2000000+01$

. 50 3八)

$.2100000+01$

-2.121)000+U1

- $21+0000+01$

$.21 j 0000+01$

-? $18000 u+01$

$.22000 .20+01$

- $22.20000+01$

. $2.230000+01$
- 1*14,1)0 $)+01$.

- 1 g(in.) ) + U 1

$.4528401)+(1$

$.5017600+01$

$.5107000+01$

$.5198400+01$
$.30 .7600+01$

$.3097600+1$

$.316 .640 u+01$

$.3240000+01$

- $3: 1 \therefore 400+01$

- $330.40+3$

- 345 , ( ) $)+1$

$.5):(34=0+01$

- jzascositis

$.5451790+r 1$

. $56.3075(i+1$

. $56=2000+01$

- $69 ? 28570+91$

- $629000+01$

$.54 .34 i^{3}(-6+)$.

-t $(-4+6,70+1) 1$

- $83-13(10)+1$

$.7(\therefore 7 \cdot 1 \cdot)+11$

$.73)(3+3)+1$

-75: (954) +1$) 1$

$.77(.2301)+1) 1$

- अ) .)(n) n+ $n+1$

- 8? $42410+: 11$

- 8.34 अ $y\left(x t_{1}\right)+1$

.5741 is $2+0 !$

-

$. \div 2 \cdots 1000+c 1$

$.05297130+11$

$.72:) 0240+61$

$.1007771)+62$

- $103 !(1: 3+2) ?$

$.10(4-3 i)+(i ?$

$.1094105+02$

$.11 ? 3(44 ?+) ?$

- $1154513+72$ 
. $2702336+02$ $.7476000-01$ - $2793410+0 ?$ $.7190000-01$ . $2897023+0 ?$ - 5021 ग00-01 - $2573220+0 ?$ -6651000-01 - $3102044+0$ ? -6387000-01 - 32. $08543+02$ - $6128000-01$ $.3317761+02$. .5874000-01

- $3429742+02$ - 5026000-01 - $3544535+0$ ? $.538 .3000-01$

- $3662186+0 ?$ $.5145000-01$ . $37827+2+02$ - 4713000-01 . $39062.50+02$ - $4685000-01$

- $4032758+0$ ? $.4453000-01$ . $4162314+0 ?$ - $4247007-01$ - $4204567+C 2$. - $4035000-C_{1}$ . $44307+6+0 \angle$ - $3929000-01$ . $4569750+0 ?$ - 36́2700 ग-01 $.4711599+02$ - $3431000-01$ - $48575.32+0:$ $.3240000-01$ -5006412+02 - 305+000-01 - $3158687+02$ . $23720(0-) 1$ $.5314410+0 ?$ - $2536000-1$ - \$47.353?+0? - $25 ? 40 \mathrm{CO}^{-0} 1$ - $563 t: 406+02$ . $2357000-01$ . 580 2783+0z - $1+5000-01$ - 5972a17+0? -?037ว00-51 -ग146560+02 - 15.34$)(U-U)$ $\therefore 324057+i$ - $173540 u-k 1$ - $.5(5.5,2)+1)$ - $15: 1$ 1 $(1)-1 ! 1$
$.10000(10+01$

$.230 n .100+01$

$.52 ;)(00+11$

$-1 \geq 1+703+3$

$.1000000+31$

$.23:(9) 100+01$

- $5 \therefore 2: 34 i n+01$

$.1273717+27$

$.1000000+01$

-2.310000+ 01

$.54756110+11$

$.2360000+01$

$.1000000+01$

$.235 i) 000+01$

$.1000000+01$

$.1000000+01$

$.24 c 0000+01$

$.1000000+01$

$.2420000+01$

$.1000000+01$

$.24400100+01$

$.1000000+01$

$.2+60000+01$

$.1000000+01$

$.2430000+01$

$.1000000+01$

$.2300 .300+01$

$.252 .0060+01$

- $1300 \mathrm{cos} 01$

$.1000000+01$

$.1000000+01$

$.1000035+01$

$.100000 .0+01$

- $1000000+31$

- $1000000+01$

- $1000000+01$

$.1000000+01$

$.1200000+i) 1$

- 12$) \div(3)+01$

-1.)00000+01

$.1000000+01$

$.100(000+1) 1$

$.1000000+01$

- 1$)(930)+3$

- 1100000)+01

- $1000(i)+21$
- $2540000+01$

$.25 \div 0000+01$

- $2530000+01$

-?600000+01

- $2.520300+01$

$.264,0000+01$

$.2660000+01$

- $2680000+01$

- 270 or $000+01$

$.2720100+01$

$.27400 .00+01$

- $2700000+01$

$.2780000+01$

- $2300000+01$

- ․: :010J+1

$.2840(100+01$.

- $2350(0) 00+01$
$.5567600+01$

$.566+400+31$

$.5760000+01$

$.5856410+01$

$.595310 u+01$

$.6051(.00+01$

.61:50400+01

$.6250000+01$

-6) $3550400+01$

$.0451000+01$

$.655 .3610+01$ 5

- $6656400+01$

-67000.)0+01

$.681 .440 .7+01$

- $696: 3600+01$

$.7 \cap 7560 u+01$

- $71\{3340 v+01$

- $72,0421+31$

$.736: 14460+31$

$.75076 .00+01$

$.7617600+01$

$.772 .8400+01$

$.7040000+31$

$.745100+61$

. $36 \varepsilon 5 \operatorname{6} 20+01$

- $317.1600+01$
- $128,1299+.72$

. $1314426+02$

- $13431 ? 7+02$

- $138 ? 400+02$

- $1417 ? 4 y+12$.

- 1 at $5: 78+02$

- $14\{8 t \cdot 24+02$

- $13.252: 99+0 ?$

- $15, ? 2.120+1 ? \therefore$

- $1600301+13$

$.16 .38736+1) ?$

- $16.777 .22+0 ?$

$.1717 .951+17$

$.17576(10+1) ?$

$.17954 ? I+13$

- $133074+1 ?$

$.1332110+02$

- $1+24: 3 \cdot 3+42$

$.176,2402+2 ?$

- $201: 33.5+32$

- $2057032+62$

- $2102.43+3+12$

-.21484:05+0?

$.214500+52$

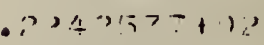

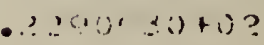

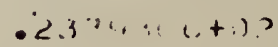


. $669.5586+0$ ? . $1452000-01$ $.6875707+02$

. $1316000-01$

. $76,72510+0$ ?

. $1185000-01$

. $7269453+02$

- $1059000-01$

. $7471182+02$

- $3360000-02$

$.7676563+02$

$.8170000-02$

$.7936150+02$

- $7030300-0 ?$

$.310000: 1+0 ?$

$.5920000-02$

. $3313170+02$

$.4850000-02$

. $3540717+0 \%$

. $3820000-02$

- $3767701+0 ?$

. $2830000-02$

$.3999179+02$

- $187000 n-0$ ?

-423521u+c?

. $7500000-0.3$

$.9475854+02$

$.70 .00000-04$

$.3721171+02$

-.750000.0-03

$.39712 ? 1+02$

-.1600001)-02

. $1022606+0.3$

- . ?38 c000-0?

$.1048576+C .3$

$. .313000 n-02$

$.1075037+03$

-.3350000-0?

$.1101996+03$

$-.4530000-02$

$.1129459+03$

$-.5190000-C 2$

. $1157432+03$

- .5820000-02

$.1185921+03$

-.5420000-02

- $12,14933+0.3$

- $0080000-02$

- 1? $44474+03$

- .75 30000-0\%

$.1274551+01$

$-.3040000-02$

$.1305169+03$

-. $3530000-02$

- $1336336+0.3$

-. $5990000-C 2$

. $1368058+03$

$-.7430000-02$
$.1000000+01$

- $2580000+01$

$.3294+101+01$

$.238 i^{3}+47+2 i$

$.1000000+0$

- $\because 900(0)+31$

$.2020000+01$

$.2940000+01$

- $2960000+01$

. $29830000+01$

$.3000000+01$

-3งนuc:0u+u1

$.3040000+01$

- $3000000+01$

$.30301500+01$

$.1000000+01$

$.1000000+01$

$.310(\because 000+)$.

- $1000000+01$

$.3120001+01$

$.1000000+01$

$.3140000+01$

$.1200000+01$

$.3160000+01$

$.1000000+01$

$.3140000+01$

-11) $000000+11$

$.10 \cos 000+01$

$.10 \operatorname{coc}(0+01$

$.1000000+01$

$.1000000+01$

$.10008(10+01$

$.1000000+01$

$.1000000+01$

- $1000000+01$

. $1000000+01$

- $1000000+01$

$.1000000+01$

$.1000000+01$

$.8410000+01$

$.2425120+02$

$.3526400+v 1$

. $248900+22$

$.8643600+01$

$.2541 \geq 18+02$

$.8761600+01$

$.2593434+012$

$.8880400+01$

$.2540359+0 ?$

- suunouotol

$.270013: 20+22$

$.9120400+01$

$.27543 \% 1+1)$

$.9241600+01$

- $38074.56+02$ ?

$.0303600+01$

. $>3 E x ?=2+02$

$.9436400+01$

$.2021311+02$

$.5(1) 000+01$

-2)7?163+)2

$.9734490+01$

- $3037133+02$

$.9853600+01$

- $3095914+02$

$.9635600+01$

- $3155+200+02$

. $1011240+0: ?$

- $3215743+0 ?$

$.1024000+0 \%$

$.3275830+1) 2$

$.1036240+02$

- $33384: 95+02$

$.1040700+07$

. $3401: 2 ? ?+0 ?$

- $106,276,0+0 ?$

$.34 t 45 x \cdot 8+1 ?$

$.1075040+02$

. $3523755+02$

$.10 \times 9000+02$

$.3593700+02$

$.11022+0+02$

. $36594.47+02$

$.1115560+02$

$.37 \div 5-70+1) ?$

$.1125360+02$

$.374 .306+32$

$.1142440+02$

- $33+1447+0 ?$

$.1150000+02$

$.3030400+0 ?$

$.1169640+02$

$.4000164+0 ?$

$.3420000+01$

$.3440000+01$

- $1183360+02$

$.4070753+c)$ 
$.1403341+03$

- $9840000-02$ $.1433192+03$

-.102300J-01 - $14 j 6<1 a+0.1$

- . $1059000-01$

- $150 J \in 25+0.3$

-.1094000-01

- $1535220+03$

-.1126000-01

- $1570410+03$

-.1155000-01

- $1606201+03$

-.1183000-01

- $1642601+03$

-. $1209000-01$

- $16.79616+C 3$

- $12-33000-01$

$.171725 y+03$

-.1255000-01

$.1755515+1) .3$

- .1?.75)00-01

$.1744421+v .5$

- $12 y 3000-01$

- $13=3 \cos C+\mathrm{C}_{3}$

-.13100010-01

- $187416.1+C 3$

- $1325000-01$

$.1915013+03$

- $1338000-01$

$.1956530+0.1$

-.1350000-01

- 14 y $8717+0.3$

- $136,0000-01$ . $2041584+0.3$

- $1369000-01$ . $2085136+03$

-.1376000-01 - $2129.381+03$

- $1382000-01$ . $2174327+03$

- $1.3837,000-01$ - $2211981+0.3$

-.1390000-01 - $2266350+03$

- $1392 \mathrm{CO} 0-01$ . $2313441+03$

- $133.3000-01$ - $2301 ? 62+0.3$

- $1353000-01$ . $2409822+03$

- $1.3 \div 2000-01$ - $2453126+03$

- 13 3 $000-01$ - $2509183+(: 3$

- 1 jiś(0)0-01 $.3560000+103$

\begin{tabular}{|c|c|c|c|}
\hline $.10000 \mathrm{CO} 0101$ & $.3460000+01$ & $.1197160+0 ?$ & $.4142174+02$ \\
\hline $.1300000+01$ & $.3430000+01$ & $.12110+0+02$ & $.4 ? 1441 \%+02$ \\
\hline $.10000(0+01$ & - 35ucir:00+01 & $.132: 5000+0 ?$ & $.42875 i 00+02$ \\
\hline $.1000000+01$ & $.3520000+01$ & $123+040+02$ & $.4361421+02$ \\
\hline $.1000000+01$ & $.3540000+01$ & - $1253160+0 ?$ & $.44 .361 \div 6+02$ \\
\hline $.1000000+01$ & $.3500000+01$ & $.1267 .360+32$ & $.4511: 302+02$ \\
\hline $.1000000+01$ & $.3530000+01$ & $.1281<10+0 ?$ & $.45653 \times 71+02$ \\
\hline $.1000000+01$ & - $3000000+01$ & $.12 \cdot 36000+02$ & - $4685600+0 ?$ \\
\hline - $1000000+01$ & $.3620000+01$ & $.131(144 !)+(12$ & $.4743 i(3 . i+c ?$ \\
\hline $.1000000+01$ & $.3540000+31$ & $\cdot 13: 24: 30)+1 ?$ & $.4322 i 344+32$ \\
\hline $.1303000+01$ & $.360 .000+01$ & $.133450)+0$ & $.4: 30279.5+37$ \\
\hline $.1000009+01$ & $.36830000+31$ & - $13: 1424 \cdot 1+0 ?$ & - $49036: 03+0 \tilde{c}$ \\
\hline $.1: 100000+01$ & $.3700200+01$ & $\therefore .1360000+0 e^{-}$ & - 5be. $5300+12$ \\
\hline $.1000000+01$ & $: 3720000+01$ & $.1383840+0 ?$ & $.5147 .4 .35+02$ \\
\hline $.1000000+01$ & $.3740000+31$ & $.1398763+02$ & $.5231362^{3}+2 ?$ \\
\hline $.1000000+01$ & $.3760000+01$ & $.1413760+02$ & $.5 .3157 .73+12$ \\
\hline $.1000000+01$ & $.37800000+01$ & $.1429340+102$ & $.5401015+0 . ?$ \\
\hline $.1000000+01$ & $.381)(1000+01$ & $.1444000+0 ?$ & $.5487 ? 00+02$ \\
\hline $.1000000+01$ & $.38261000+01$ & $.1459240+0 ?$ & $.5574 ? 7+v 2$ \\
\hline - $1000000+01$ & $.3840000+01$ & $.1474560+v \because$ & $.5 t 62311+j \%$ \\
\hline $.1000000+01$ & $.3360000+01$ & $1484 \times 3<0+0 ?$ & . $573124+\cdots+11$ \\
\hline $.1000010+01$ & $.3830000+01$ & $.1505440+02$ & $.5841267+11 ?$ \\
\hline $.1000000+01$ & $.3900000+01$ & $.1521000+0 ?$ & $.5,31>0.0+0 ?$ \\
\hline $.1000000+01$ & - $3 \div 0000+01$ & $.1536 .640+02$ & 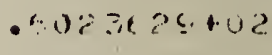 \\
\hline $.1000000+01$ & $.3940000+01$ & $\cdot 1552360+(12$ & $\cdot \dot{11} 16 \Rightarrow \Rightarrow+0 ?$ \\
\hline $.1000 \mathrm{COO}+01$ & $.3560000+01$ & $.156 \% 100+0 ?$ & - $\operatorname{t}() \div y 14+0 ?$ \\
\hline $.1000000+01$ & $.39800130+01$ & $.1584040+0 ?$ & - $(3)+47 \because+03$ \\
\hline .11$) 00000+01$ & $.4000000+11$ & $1600000+0 ?$ & $.3400000+00$ \\
\hline
\end{tabular}


HP13: POL YNOMIAL 201

.000000

- 00100000

- 1960000-01

- 0000000

$.3842000-61$

. 0000000

. 5647000-01

. $1000000-04$

. $7377000-01$

. $4000000-04$

- $9033000-01$

. $1000000-0.3$

$.1061800+00$

- $2100000-03$

- $121.3100+00$

- $3800000-03$

$.1357600+00$

. $6600000-03$

$.1455400+00$

- $1350000-02$

- $1626.600+00$

- $1600000-02$

- $1751300+00$

- $2.340000-02$

- $1369800+00$

-3320000-02

- $1382200+00$

-4570000-02

. $2088700+00$

- $6150000-02$

- $\therefore 183300+00$

- $3100000-02$

- $2284200+00$

- $1349000-01$

$.2373700+30$

- $1336000-01$

- $2457700+00$

- $1680000-01$

-?53660út00

- $2085000-01$

- $2010300+00$

- $2560000-01$

- $367.02 .00+00$

- $3112000-01$

$.274 .32 .00+00$

- 374 ห०००-01

- $2302001+00$

. $4477000-01$

- $2857400+00$

$.5308000-01$

.?907+00+00

- $6.250300-01$

- $2754100+00$

- $7312000-01$

. $2970100+00$

APPROXI MATION PROBLEY

$.1000000+01$

- 0000000

$.1030000+01$

. 0000000

$.1000000+01$

.0000000

$.1000000+01$

.0000000

$.1000000+01$

.0000000

. $1000000+01$

- $1000000-04$

$.1000000+01$

.2000000-04

. $1000000+01$

. 50c0000-04

- $1000000+01$

$.1000000-03$

- $1000000+01$

- $1900000-03$

$.1000000+01$

- 320000 0-03

$.1000000+01$

- 52.00000-03

- 1000000+01

- 80c0000-03

- $1000000+01$

- $1190000-02$

- $1000000+01$

- $1720000-02$

- $1000000+01$

- 2430000-02

- $1000000+01$

. $3360000-02$

. $1000030+01$

- $4540000-02$

- $1000000+01$

. $6050000-02$

- $1000000+01$

- 7020c0u-02

- $1000000+01$

- $1024000-01$

$.1000000+11$

- $1307000-01$

$.1000000+01$

- $1049600-01$

- $1000000+01$

$.2000000-01$

- $1000000+01$

. $2543(01)-01$

$.1000000+01$

. 3125c010-1

- $1000000+01$

- 3302:00 J-21

$.10000(5)+01$
.000 nunn

$.2000000-01$

$.4000000-0: 4$

- $10 \div 0 n 00-04$

$.4000000-01$

$.1600000-02$

$.6000000-04$

$.6000000-01$

$.3600000-02$.

$.2200000-03$

$.8000000-01$

$.6400000-02$

$.5100000-03$

$.1000000+00$

$.1000000-01$

$.1200000+00$

$.1440000-01$

$.1400000+00$

$.1060000-01$

$.2560000-01$

$.1600000+00$

$.1800000+0 C$

$.3240000-01$

$.2000000+00$

$.2200000+00$

$.2400000+00$

$.2600000+00$

$.2800000+00$

$.3000000+00$

$.3200000+00$

$.3400000+00$

$.3600000+00$

$.3830000+00$

$.4000000+00$

$.4 ? 00000+00$

$.4400000+00$

$.4600000+00$

$.4800000+00$

$.501) 0600+00$

$.5200000+00$

$.5400000+00$
$.4000000-01$

$.4340000-01$

$.5760000-01$

$.6760000-01$

$.7840000-01$

$.9000000-01$

$.1024000+00$

$.1155000+00$

- $1296000+00$

$.1444000+00$

$.1600000+00$

$.1764000+00$

$.1636000+00$

$.2116 .000+00$

$.2304000+00$

$.2500 \cup 0 c+c c$

$.2704000+00$

$.2916000+00$
$.1000000-0 ?$

$.17300011-0 ?$

$.2740000-02$

$.4100 j 00-0 ?$

$.58 .300110-002$

$.801,0000-02$

$.1005000-01$

- $1332000-01$

$.1750000-01$

- $2145000-01$

$.2700000-01$

$.3277000-01$

$.3930000-01$

$.46 E 6000-31$

$.54 .37(10.7-01$

$.6400000-11$

$.740030 .0-01$

$.8518000-11$

$.9734001)-01$

$.1105500+00$

- $1250000+30$

$.140 F 100+00$

$.1574200+00$ 
- $\pm 50.3000-01$

- $3034200+00$

. $\because 334000-01$

- $3053400+00$

$.1131600+3)$

$.3098800+00$

- $12960(\mathrm{~J})+\mathrm{CO}$

- 3125 toutuo

- $1477000+00$

$.3149000+J C$

$.1677700+00$

$.3163900+00$

$.1897500+00$

$.3185000+00$

- 2.133100+OC

$.3159100+00$

. $2401000+00$

. $5209600+00$

$.26837403+00$

$.3217100+00$

.:998700+00

- $32 ? 1300+00$

-.3336200+0u

$.3223300+10$

$.3701500+00$

. $\therefore ? 2.3 .30 u+00$

$.4040000+00$

$.32202(0)+00$

$.4521200+00$

-j214700+ 00

. $4378700+00$

. $3206900+00$

.5470100+00

$.31963\left(0^{\prime}\right)+130$

. $53970(0)+0$.

- $3194800+20$

-

$.3170000+00$

$.7163903+30$

$.31545(10)+U 0$

. 7807500+v0

- 31 scuootuo

- $34+3503+01$

$.3110900+00$

. $223700+00$

- is.3560n+00

$.1002003+3$

$.3072700+(10)$

. $10+324.30+01$

- 304.1200+00

$.1163860+01$

- $: 23.300100$

- $12624830+(11$

. $3905(13) 0+.15$

- $136: 0440+01$

- :3r. $36(000+) 0$

$.1454100+01$

$.29 .30400+100$
$.4592000-01$

- $1000000+01$

- E507000-01

. $1300(100+01$

.6564):00-J1

- $1000 j 00+01$

$.7770000-01$

- 1) $00000+01$

$.91 f_{3} 1000-01$

- $1300000+01$

$.1073700+00$

$.1000000+01$

- $1=52500+00$

- $1000000+01$

$.1453900+00$

- $1100000+01$

- $1(.8) 700+0$ ?

- $1000000+01$

$.14344(31)+0$

- $1300000+01$

- $2213,500+013$

- $1000000+01$

$.2535 .560+06$

$.1000000+01$

- $238>200+00$

- $1300.000+01$

- 327000010

- $1000000+01$

- $.3707400+00$

- 1 i) $50000+01$

- 41 Ealuvau

- $1000000+01$

$.4714300+00$

- 1 njo00jutni

- $5277300+(0)$

- $1000000+01$

$.5904900+00$

- $1000000+01$

$.659903150+01)$

- $1200000+01$

$.733+c 00+00$

- $1000000+01$

- 315\$3700+03

- $10006160+01$

$.00 .392 .00+0 a$

- $1000000+01$

- $1100000+01$

- 10 cusiciotu 1

$.11040 ; 30+01$

- $1000000+01$

- $12166.50+01$

- $10 \mathrm{cos}(100)+01$

- 1 3332.35+01

- $1000 \mathrm{cos} 01$

$.1703330+01$

. $1000(j)+01$

. 161$) 510+01$

$.10000(0)+01$
$.5000000+00$

$.7136000+0$ )

$.175320 n+00$

- 5ษงucoutoo

$.5364000+0$

$.1251166+90$

$.5000000+00$

$.30010000+6$

$.2150,00+20$

$.6200000+00$

$.3844000+001$

$.64006,00+00$

$.4000000+00$

$.6600000+00$

$.43560(10+0$.

- $6306(100+00$

$.7000000+00$

$.7200000+00$

$.7400000+00$

$.7600 .000+00$

$.73000: 00+00$

$.9000000+00$

$.8210500+00$

$.34006,00+00$

- उ60r000+00

$.8900000+00$

- $\rightarrow 00000+00$

$.5200000+00$

$.94002010+00$

$.03000000+00$

$.9900000+00$

$.1000000+01$

$.1020000+01$

$.1040000+01$

$.10() 000+$.

$.1(1-31): 1050+01$

$.1100000+01$

$.122^{2} 0(120+01$
$.4624000+00$

$.4900000+00$

$.5180 .0000+00$

$.5+7: 11) r: 0+(\cdot)$

$.5770100+j=$

$.808(4.100+v i$

- $134000000+00$

$.6724000+00$

$.7050000+00$

$.7330000+21$

$.77+4300+01$

$.8100000+00$

$.84240010+100$

- $23.360 .000+0)$

- $921(10) 00+00$

$.96041)(10+0)$

$.1000000+02$

$.1040400+31$

$.1081(i) c+01$

$.1123600+01$

$-1160400+31$

-12.100(n)+a!

$.125+4,3(3+$. $\because 21.32 \div 0+00$

$.2621+00+00$

- $375000+00$

$.3144-i(1)+(10$

- $543(2)(0)+i) u$

- $, 7.325,01+4$

- $A(0, \because, ? \cdot 1)+r 0$

$.4 \div: 9+20+23$

$.+745 \div 17(1)+3$

$-5120(100+00$

$.5513700+n j$

- $59270: 30+1)$

- $(36050(\div+)$

- 6) $1470 i+0 ?$

$.728(0) 00+1: 0$

$.77(.5(5) 6+0)$

- $230.50,1)(1+(14)$

- $583+i 41)(1+(1)$

$-14+11 \cdots, 6)+00$

$.1000 .010+1,1$

- $10 \% 1 ? 10+11$

- $\mid 1(2+(+(i)+1) \mid$

- $11+1 j:(3+01$

- $1 \because r .1713+1) 1$

- $13 \div 1311011$

- $1(1(1)+(3)\{3+11$ 
$.1573520+01$ $.2906000+00$ $.1638961)+01$ - $2974100+00$ . $\left.131064^{\prime}\right)+01$ . $2841100+00$ - $19.38780+01$ . $2307200+00$ $.2073600+01$ . $2772500+30$ $.2215330+J 1$ $.27369 \mathrm{CO}+10$ . $2364210+01$ $.2700600+00$ $.252 .0470+01$ . $2603600+00$ - $26: 4450+i 1$ - $26.26000+10$ - $2856100+01$ - $2587900+20$ - $\therefore 035300+01$ . $54+9000+j 0$ - $32 c^{2}+190+01$ -r.507500+00 . $2421020+11$ .?+7010u+00 - $3(? 674)+01$ . $2430100+0.0$ . $\$ 841600+01$ . $2333700+00$ . $4065870+01$ - $343000+00$ - $+248320+01$ $. ? 308100+(i)$ $.4543720+01$ $.2207000+00$ . $4797850+01$ . $2225700+00$ .?0.5250J+01 $.2184300+c 0$ . $5337950+01$ . ?142300+00 - $56: 24490+01$ - $2101200+00$ - $5922410+01$ - L0 jy $700+00$ - $2.32010+01$ $.2013100+c 0$ - $5553600+01$ $.1976 .600+00$ - $(8 t i 7480+1) 1$ . $1935200+00$ $.7233950+01$ $.1333800+00$ $.7593330+01$ $.1352600+60$ $.7705340+01$ $.1811500+00$
$.1762340+01$

. $1000000+01$

- $1935410+01$

$.1000000+01$

. $2100340+01$

$.1000000+01$

. $22.87760+01$

$.1000000+01$

$.2488320+01$

$.1000000+01$

$.2702710+01$

. $1000000+01$

$.2931630+01$

$.1000000+01$

. $3175830+01$

- $1000000+01$

$.3435: 71+01$

- $1300000+01$

$.3712930+01$

$.1100(0)+01$

- $413 \cup 7460+J 1$

- 1 ن $0000+01$

.432.) 430+01$

-1.) 0n000+os

. $4052500+01$

- $1000000+01$

$.5004900+01$

- $10000(10+01$

$.5373240+01$

- $1000000+01$

$.5773530+01$

- $1000000+01$

$.6191740+01$

- $1000000+01$

- $(6 ; 3 ; 330+01$

- $100(000+u 1$

$.7100320+01$

- $1000000+01$

- $7553750+01$

. $1000000+01$

- $8113680+11$

- $1000001+01$

- se6171u+u1

- $1000000+01$

$.9233560+01$

- $1000000+01$

- $9840500+01$

- 1$) 00000+01$

- $104357 \varepsilon+0$ ?

- 1$) 00000+01$

$.1115771+02$

. $1000000+01$

$.1186363+02$

$.1000000+01$

- $1260493+02$.

- $1030000+01$

- $133322^{7} 5+02$

- $1000000+01$
$.1140000+01$

$.1299000+01$

$.1481540+J 1$

$.1160000+01$

$.1345600+01$

$.1180000+01$

$.1392400+01$

$.12000100+01$

$.1440000+01$

$.1220000+01$

$.1483400+01$

$.1240000+01$

- $1537600+01$

$.1260000+01$

$.12901000+01$

$.1300(10)+$.

- $1321.101+91$

$.1340000+31$

$.1350(1) \cup 1+01$

$.13300: 00+01$

$.140(0) 00+01$

$.1+20000+01$

$.1440(0)+01$

$.14(00000+01$

$.1450000+01$

$.1500000+01$

$.1520000+01$

$.1540000+01$

$.1560000+01$

$.1533000+01$

$.160(1000)+01$

$.10201000+01$

$.16400000+01$

- 1 etconouto 1

$.108(000+01$

$.1700000+31$
-15:170.00+01

$.1(354) 0+C 1$

$.18 .000000+01$

$.174 a 40 v+01$

$.17) 5000+01$

$.13450000+c .1$

$.1904400+01$

$.1960000+01$

$.2010400+01$

$.2073000+01$

$.2131600+01$

$.2100400+01$

$.2250000+01$

$.2310400+.11$

$.2371000+01$

$.24336500+01$

$.2496409+01$

$.2560 .300+01$

- $2 \operatorname{tat} 40 n+01$

$.2639600+01$

$\therefore 75=000+01$

- 2426400+01

$.2800000+01$
. 15 equdotod

$.1643 .3)+C=1$

- $1728100+01$

$.1015050+01$

$.190(.6-2)+31$

$.20003(17)+1$

$.2007150+1$

$.21(47007+11$

$\therefore 2909 \% 2+21$

$.2406100+01$

$.2515460+11$

- $30207 j+n 1$

$.27+4000+j 1$

- 235320$)+31$

. $3735(52)+3$

$.3112141+31$

$.3241745+11$

$.13751000+01$

-351 $1310+31$

$.3736 .423+02$

$.304431 .1+11$

$.40 .36(a r)+31$

-452) 153,3$)+1$

$.4+13(340+01$

$.45+7+2.3)+31$

$.47410 .30+11$

$.4013001+1$
- 36) $2200+31$ 
- $3352100+01$ . $17708 \mathrm{CO}+00$ . $3752130+01$ $.1730100+00$ - $11<5300+21$ . $1689700+2)$ . $4555130+61$ - $16: 4+50 j+00$ - $1003376+02$ . $1509.900+00$ - $1049760+02$ - $1570200+C 0$ - $10971913+0 ?$ - $1531000+00$ - $1146224+0 ?$ - $1432100+00$ - $1130083+02$ - $1453500+00$ - $12491483+C=$ - $1415+00+00$ - $1303210+0 \%$ . $1377 \mathrm{c00+00}$ - $1358955+0 ?$ - $1.340200+00$ - $1416469+02$ - $130320 \mathrm{u}+00$ - $1475799+C$ ? - $1266700+00$ - $1536 y 54+02$ - $12.30000+00$ - $1600000+0$ ? $.1195000+00$ - 1 É 64 ;E 6 +C 2 $.1159330+00$ $.1731371+02$ - $1125000+00$ - $1800-314+0 ?$ - $10,0300+00$ - $1871774+02$ - $1057100+i 7$ - 1344 is $10+02$ - $1023800+00$ - $2014563+5$ ? - $+1 \cup 0 \mathrm{C} u-01$ - $27972.74+0.2$ - $\therefore 53+300-01$

- $3176783+v_{2}^{2}$

- 3271000-01

- 2? 5\{5-31+0?

- $1953000-01$

- .234?560+02 - śe $51000-01$

.? ? $23913+4$ ?

- :350001-01

- $2517,31+12$

- (3) 53000-ci

- "Re JR>shro?

$.776 z . j r a-01$
- $1419 \varepsilon j た+U ?$ - 1 J0( 00$)+31$

- $150 \div 3 \varepsilon .5+0 c$. - 1$) G 0000+01$ - $15 y+947+02$ . $1000000+01$ - 1 c சன $742+02$ - $1000000+01$

- $1730399+02$ - $1000000+01$ - $1389563+0$ ? - 1.)0uvout01 - $1596403+0 ?$

- $1000000+01$ - $2107061+0 ?$

- 1!)0000J+01

- 2220203+02

- $100000+01$

- 2.34 34.73+i?

- $1300000+01$

- $2474.890+32$

- $1000000+01$

- 2(i) $193+3$

- 11)0000 01 -2\%47'+44+0? - $1000000+11$ - :39 $45547+0 ?$ - 1!)000(3+01 - 30431 bat - $1000000+01$ - 7200600+0? - $1000000+01$ - $-1363232+02$ - $1000000+01$ - $3531,59+0$ ? - 10$) 20(0+01$ - $3705677+0$ ? - $1000000+01$ - $335.323 y+02$ - 1 ว ग00)+01 - $40 E 4101+02$ - $1000000+01$ - $4262 z 22+0 ?$ - $1000000+01$ - 442,313is+0? - 1 u ul 00otol -47.01850+32 - $1000000+01$ - $4423597+02$ - $1000000+01$ - $5153632+02$ - $1000000+01$ -535? 1.30+??

- 1 inoocratol - cos $5+4 y+0 i$

- 10 jol $000+01$ - Sr 3ta $3+1)=$ - 1.)00000+01

\begin{tabular}{|c|c|c|}
\hline $.1720000+01$ & - $? 96,34+12+31$ & $.50380+2,0+53$ \\
\hline $.17+0: 00+01$ & $.39: 276.10+01$ & - $52(\cdot .3,1)+1$ \\
\hline - $1760100+01$ & $.309700^{\circ}+01$ & $.5451700+21$ \\
\hline $.1780000+01$ & $.3168400+01$ & .503975)+01$. \\
\hline $.1300020+01$ & $.3240000+131$ & $.533 \geq 000+01$ \\
\hline - $1320(00+01$ & $.331 r+400+01$ & 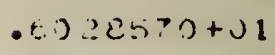 \\
\hline $.13401000+01$ & - 3.5 सEE (x) $00+1 ! 1$ & $(6,2=2(2)(1)+1) 1$ \\
\hline - 1 (sti, ()$\left._{1}\right)+01$ & $.345 .3500+.31$ & $. t 4.742 . .1)+11$ \\
\hline - $1 \sec (0) 00+01$ & $.353+4.20+61$ & - foit $_{4}=1:+, 1$ \\
\hline - $1+i \log (0)+i 1$ & $-3 \in 1(1,00) 0+01$ & - $6+i+1, c+c 1$ \\
\hline - $1920100+01$ & $.3 E \varepsilon .4 c u+11$ & $.7077 \cdot 1,+11$ \\
\hline $.134(n) u 0+1$ & $.376 \cdot 3 t)(10)+u 1$ & $.73) 1 ; 30+11$ \\
\hline $.13(3) n 20+01$ & $.3841600+01$ & - $75,2: 05,40+111$ \\
\hline $.19 .5(1.0 .00+01$ & $.3420400+01$ & $.77(\cdot 23: 00+61$ \\
\hline $.2000000+01$ & $.4000000+n 1$ & - $50010+4:$ \\
\hline $.2020000+01$ & $.4080400+01$ & - $524>4: 1+1$ \\
\hline $.204(1,0)+?) 1$ & $.416,1000+01$ & $-14:(3), 1)+11$ \\
\hline - $2060000+01$ & $.424 .3000+01$ & $.3741+20+01$ \\
\hline $.20 .30000+01$ & $.4320: 400+01$ & - 30y $3410+01$ \\
\hline .21$) 0(100+01$ & $.4410300+01$ & - $? 351300+2)$ \\
\hline - $21200 v 0+01$ & $.44444(0+01$ & - \\
\hline $.21+0000+01$ & $.457561 ; 0+01$ & $-4001)+(1+\div 1$ \\
\hline - $21 \operatorname{coc}, \cos 01+01$ & $.46+500(i+0) 1$ & $.14,77 \cdot 6+\cdot 2$ \\
\hline - $215(1) 01$, & $.475, ? 400+(1$ & $.1036,1+9+1)$ \\
\hline - : $\because 000000+01$ & $.48410000+i) 1$ & $.10440+i$. \\
\hline$-222(0)+01$ & $.4523+0 u+11$ & $.104+165+(12$ \\
\hline - $2240 ; 010+01$ & $.50) 17 i(0)+01$ & $.11 ? 3 \cdot 0+\ldots+0$, \\
\hline - $\therefore \therefore(\cdot, 100+0)$ & $.510701 .0+C 1$ & $.1154: \because 13+6$ \\
\hline$\{3(0)(10)+1)$ & $.51054018+01$ & $.11+8,5+3$ \\
\hline
\end{tabular}


- $2702336+02$ . 7476000-01 . $2798410+02$ - 7190000-01 - 2877023+02 - $6921000-01$ - $2398220+0 ?$ - $0 E 51000-01$ - $3102044+02$ - E387000-01 . $3203543+02$ - F) $123000-01$ - $3317760+i 2$ - $5374000-01$ - $3429742+02$ - 5626000-01 - $3544535+02$ - 5383000-01 - 3f $62186+02$ $.5145000-01$ - $3732742+02$ - $4913000-01$ - $3906.250+C ?$ -4j45J0-01 . $4032758+02$ - $4+33)(2-) 1$ - $410: 2314+2: 2$ - $4 ? .470(0-1) 1$ . $429496,7+02$ - 4035000-01 - $4430766+02$ . 3329000-01 $.4569760+02$ - $20270 \mathrm{Cu}-01$ . 4711 3 - $34.31 \mathrm{ClO} 0-\mathrm{Cl}$ - $4357532+02$ . 3240000-01 . $5006412+0$ ? $.3054000-01$ - $158637+02$ - i $72000-01$ - $314410+0 ?$ - $\because 6 y 600,3-41$ - $5473632+02$ - $524000-01$ - 5630 406+0ź - $2357000-01$ - s3u2783+02 - $\therefore 1 \cap 5000-01$ - 59728817+0? - $2037000-01$ . $5146.5 E 0+02$ - $18.340(\cdot)-1$ - $324 u t 7+0$ ? - $1735: 0 j-01$ - $=505330+c$ : $.15,01000-01$
$.6161327+02$ - $1000000+01$ - $6^{\prime} 436.34 .3+0 ?$ - $1000000+01$ - $6,721003+i 2$ ? - $1000 \mathrm{coO} 01$ - $7015834+02$ - $1000000+01$ - 73220325+02 - $1000000+01$ - $763633 ?+02$ - $1000000+01$ - $7362624+0 ?$ - $1000000+01$ - $8295576+0 ?$ - $1000000+01$ - $364866 \theta+02$ - $1000000+01$

- $50083 \leqq 78+02$ - $1000000+01$ - $\$ 381200+0 ?$ - $1000000+01$ - $97656.25+02$ - $1200002+01$ - $1 \cup 16,3,3+0 . j$ - $123 j(1) 0+01$ - $1: 57,23+J$ - $1000000+01$ - $10 \cos 12+03$ - $100000+01$ $.114 .3139+03$ - $1000000+01$ - $1188134+03$ - $1000000+01$ $.1234544+03$ - $10000(0+i) 1$ $.1282383+03$ - $100000 u+01$ - $1331705+03$ - $1000 \mathrm{cco}+01$ - $1392523+73$ - 1 i) j0v0u+u 1 $.14348+1+03$ - $1000,000+01$ - $148352 i 2.3+0.3$ - $1300600+01$ - $154+375+0.3$ - $102000 ?+01$ - 16015 t3+0.3 - $10000 j 0+01$ - $16 ; 0,04+3+03$ - $1000000+01$ $.1721037+03$ - 1,00007$)+01$ - $1783337+03$ - $1000030+01$ $.1847531+0.3$ - $1.0001800+j 1$
. $2.3000000+01$

$.5250000+01$

- $1 ? 16>00+0 ?$

$.2320000+01$

$.5332400+01$

. 12.43717+1?

$.23400 .00+01$

$.5475000+01$

$.1281240+02$

$.2300000+01$

$.5569600+01$

$.1314426+12$

$.2330000+01$

$.56(.4400+01$

-1344127+??

- $3400000+01$

$.5760000+01$

$.130 .400+0 ?$

$.2420000+01$

$.2447000+01$

$.2400000+01$

$.2430060+01$

- $2501000+01$

$-2.523(1000+21$

. $20411,00+01$

$.250 .0(100+21$

$.2530000+01$

$.2600000+01$

- $2620000+01$

$.26401100+01$

- $2600000+01$

$.2650000+01$

$.2700000+01$

- $27 ? 0000+01$

$.2740000+01$

$.276 .01 .00+01$

$.2730000+31$

$.2300000+01$

- $2 \times 3 ?(1900+)$.

- 28.7$)(100+01$

- ?. $3511099+01$
$.535 t-400+01$

$.5053500+.1$

$.0051600+: 1$

. $(15) 42 n+i 1$

- (1).50) $(10+01$

-6. 35$)+10+01$

$-6+51301)+02$

. $6553 \leqslant 00+71$

. $6556400+01$

$.6760000+01$

- $\operatorname{se} 4400+01$

- $69696(1)+01$

$.7075600+01$

$.713200+01$

$.72 \div 0000+01$

$.73 \cdot 83400+C 1$

$.7507000+01$

$.7617000+01$

$.77: .840)+1$

$.7840000+01$

$.74+1040+0$

$.80(i 5,502+0)$

$.31750 .1 \mathrm{j}+01$
$.141724 \cdot 1+13$

$.14525^{\prime} 3+12^{2}$

$.14338 \dot{1}+4+13 ?$

- $152520(3+) 3$

$.1562,83+9$

$\cdot 1$ 1, J) 301+13

$.163375(5+1) ?$

$.1677722+12$

$.1717301+12$

$.175,7(, 1) 0+12$

- $1738473+i)$

- $1330774+0 ?$

- $1392110+12$ ?

$.17248 .23+1$,

- $15 \cdot(3)+12+1$

- $3012356+30$

- $205>1)+2+3$

- $210 \geq 4,53+0 ?$

- $214-3+495+12$

.21 y $300+13 ?$ ( $\because 42, ? ?+0)$

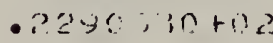
.?3+i $3+13+2$ 
$.6690586+02$ $.1452000-01$ $.6379707+02$ $.1316000-01$ $.7072810+0 ?$ $.1135000-01$ $.7265950+02$ .1059 COJ-01 $.7471192+02$ - $9360000-02$ $.7676563+02$ $.8170000-02$ $.7886150+02$ $.7030000-02$ $.8100000+02$ $.5920000-02$ $.3318170+0 a$ . 4850000-0? - $8540717+0$ ? . . $20000-0 z$ $.8757701+02$ . $2830000-02$ $.3999179+02$ $.1870000-02$ . $3235210+02$ $.3500000-03$ $.9475354+0$ ? $.7000000-04$ $.9721171+02$

-.7800000-03 $.9971221+02$

-.1600000-02 - $1022606+03$

-. 2330000-02 $.1048576+03$

-. $3130000-0$ ? $.1075037+03$

-.3850000-0? $.1101596+03$

-.4530000-02 $.1129459+13$

$-.519(00)-02$

$.1157432+3.1$

-.5820000-0?

$.1185921+0.3$

- .6420000-02

$.1214933+03$

- .6980000-02.

$.1244474+.33$

-. $7530000-02$ $.1274551+01$

-.5040000-02 . $1315163+03$

-. $3530000-02$

- $1.3 .36 .3 .3 n+03$

- . .399000u-0? $.136,3053+0.3$ $-.94 .300 C$ 0 -0?
$.1013507+03$ $.1000000+01$ $.1931356+03$ $.1000000+01$

$.2051115+03$

- $1000000+01$

$.2122825+03$

- $100000.0+01$

- $2196528+03$

- $1000000+01$

- $2272203+03$

- $1000000+01$

. $2350073+0 j$

$.1000000+01$

$.2430000+03$

- $1000000+01$

. $2512: 087+0.3$

- $1200000+01$

- $2596378+03$

- $1000000+01$

- $2082516+03$

- $1000000+01$ $.2771747+03$

- $1000000+01$

-? $242315+03$

- 1 jouj000+01

- $2350407+03$

- $1000000+11$

$.3052448+03$

- $1000000+01$

. $315: 906+03$

- $1000000+01$

- $3251935+03$

- $1000(000+1) 1$

- $3.35544 .3+03$

- $1000000+01$

- $3401 \in 20+03$

- 10000$) 0+01$

- $3570457+03$

$.1000000+01$

$.3682036+03$

- $1100000+01$

$.3796370+03$

- $1000000+01$

. $33135.19+03$

. $1000000+101$

. $4033578+03$

$.1000000+01$

$.4150544+03$

$.1000000+01$

- $4282490+03$

. $1000000+01$

$.4411472+03$

- $1000000+01$

$.45431242+03$

- $1000000+01$

.4. $78758+03$

- $10000 u 0+01$
$.2680000+01$

- $3254400+111$

$.3+1(0)+31$

- $300000+31$

$.2920000+01$

$.3526 .400+01$

$.80431500+01$

. 2940000+01

$.296000 .0+01$

$.2930000+01$

$.3001000+01$

$.3020000+01$

$.3040(00+i) 1$

$.3360000+01$

$.308000 u+01$

$.3101000+01$

$.3130000+01$

$.3140000+01$

$.3100000+01$

$.3182000+01$

$.3200000+01$

$.3220000+01$

$.3240000+01$

$.3200000+01$

- $3230000+01$

$.3300000+01$

$.3320000+01$

$.3340000+01$

$.360000+01$

$.3380000+01$

$. .3400000+11$

$.3420000+01$

$.3440000+01$
$.876 .1<00+01$

$.838) 400+01$

$.9000000+0 !$

$.9120400+31$

$.92 .41600+01$

$.9 \pm t .3600+0)$

$.9436400+01$

$.5010 .300+01$

$.9734400+01$

- $9859600+01$

- $5985600+01$

$.1011240+02$

$.10: 40) 0+2 ?$

- $103,5340+0 ?$

$.1049700+0 ?$

$.1002760+1) \%$

$.1075 \div 40+05$

$.108370170+02$

$.1102240+0 ?$

$.1115500+0 ?$

$.11 ? 3960+02$

$.11434 \div 0+0 ?$

$.1150000+0$ :

- $116 \div 6,40+0 ?$

$.1125360+06^{2}$
- $2.32: 37: 37+02$

- $2438,107+1 ?$

- $2.48: 7703+02$

. $2541 ? 13+1 ?$

- $25.533424+12$

- $2046355 r+7 ?$

$.27,0: 100+32$

- $2754361+02$

$.2300446+12$

- $2 r\left(j c^{2}(2+) 2\right.$

- $421311+32$

$.0579100+02$

. 3.) $=71 \div 3+02$

. $3055914+12$

$.7155450+3 ?$

$. .3215743+0 ?$

- $3275300+02$

$.333 \cdot 3(2.95+9) ?$

-. $3401222+32$

- $34 f \cdot 45613+0 ?$

$.352 .3795+0.2$

- 350 (7) $)+32$

- $365094: 7+) ?$

- $37: 34,9+0 ?$

$.37,3305+27$

$.386 .1447+02$

$. .19 .3 \cup 431+3.3$

$.40(3) 15 \div+i . ?$

$.4 J 7075+3+2$ 
$.1400341+0.3$

- $9840000-0 ?$

$.1433192+03$

- $1023000-01$

$.1460 E 10+1) ?$

- $103906 v-v 1$

$.150060^{2} 5+03$

- .1094000-01

$.1535220+03$

-.1126000-01

. $1570410+03$

-.1155000-01

. 16 ग52. $1+0.7$

- $1183000-01$

$.1642601+03$

-.1207000-01

$.1679616+03$

-. $1233000-01$

$.1717253+03$

-.125500u-01

$.1755519+03$

-.1275000-01

$.1794421+03$

-. $1233000-01$

$.183396 .6+C 3$

-.131000n-01

$.1374161+03$

-.1325000-C1

$.1915013+0.3$

- $1338000-01$

$.1956530+03$

-.1350000-01

$.1598717+03$

-.1360000-01 $.2041584+\mathrm{C} 3$

-.1369000-01 $.20351 .36+13$

-.1375000-01 .$\geq 129331+0.5$

- .1332000-01 . $2174327+03$

- $1357000-01$

- 2? $19981+03$

- 139 cuoj-01

- $2265350+03$

- .1392000-01

. $2313441+03$

- $1393000-01$

- $2361262+03$

- $1393000-01$

$.2409822+03$

- .1392000-01

$.2459126+03$

-.1339000-01

$.2509183+0.3$

-.1395000-01

$.2560000+0.3$
$.481717 .3+0.3$

- $1200000+01$

$.4558 \cdot 34 j+03$

. 1 UC ( $0010+1$

$.51033 .30+0.3$

$.1000000+01$

- 5.3521 $75+0.3$

- $1000000+01$

$.5403975+03$

. $1000000+01$

$.5559251+03$

. $1000000+01$

$.5718077+0.3$

- $1000000+01$

. $5880512+03$

. $1000000+01$

$.6046618+0.3$

. $1000000+01$

. $6216455+03$

- $1000000+01$

- 6390030003

$.10)(000+01$

$.6567531+03$

- $11000.30+01$

- $t 743595+13$

- $1000000+01$

- $6.534,3,6+03$

- $1000000+01$

$.7123840+0.3$

- $1000 \mathrm{coot01}$

$.7317421+03$

. $1000000+01$

. $7515177+03$

- 1 joccojoci

$.7717137+03$

- $1300000+01$

$.7423517+03$

$.1000000+01$

- $8134 ? 37+03$

- $1300000+01$

- $8349417+03$

- $1000000+01$

- $3562126+03$

- $1000000+01$

$.8703437+0.3$

- $1000000+01$

$.9022420+03$

- $1000 \mathrm{cul} 0+01$

- $9256149+03$

- $1000000+01$

$.9494697+03$

. $1000000+01$

- $9738133+03$

- 1 juodorjot 1

- $5 y 86547+03$

- 100300$)+01$

$.102+0.10+04$
$.340(00)+21$

$.3+30000+01$

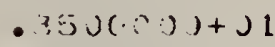

- $3520000+01$

$.3540000+01$

$.3501000+01$

. 35 RUNOOO+O1

$.3600000+01$

$.3620000+01$

$.3640000+01$

$.360 .3000+01$

- $3630(0.50+01$

$.370(: 000+01$

$.372 .0(1) 0+01$

$.3740000+01$

$.37506 .00+01$

$.3780000+01$

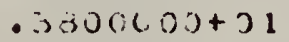

$.383000+01$

$. .33+10000+01$

$.3860600+01$

- $3800(00)+01$

$.500000+01$

$.3420000+01$

. $3440000+01$

$.3960000+01$

$.3380003+1$

$.4000000+01$
$.11971 \div 2+u ?$

- $12110+i+0$ ?

. $13: 3.195+03$

-1?.3.7040+0?

. $12.53100+0 ?$

- $1267300+102$

$.1281040+02$

- 12 atouto

$.1310440+02$

$.1324(3) 0+0 \%$

$.13315 \cdot 0+112$

- $1354 ? 40+0 ?$

$.1369000+02$

$.138 \div 640+0 ?$

$.1398760+02$

$.141376,0+0 ?$

$.1428(340+12$

$.14443(0)+0 ?$

$.1459240+0 \%$

$.14745 .50+0 \%$

$.14: 3 \rightarrow(36)+C_{2}$

$.1505442+0)$

$.1521600+0 ?$

- $1535,643+6$

$.1552750+02$

- $1505160+02$

$.152 .4040+3$

$.1600000+3 ?$
$.4142174+3 \%$

$.42 .1441 \%+32$

- $42\{79017+2\}$

$-43614 ? 1+6 ?$

$.44361 \cdot 36+0 ?$

$.+511822+02$

-45:3\&?? $1+62$

$.46+5000+i 2$

$.474 .3 ? \div 3+\cup 2$

.48??354t??

$.490 ? 70 u+3$

. $498.303+32$

. รด55.30 0+0?

$.5147385+C ?$

$.523136 ?+02$

$.5315 .738+1 ?$

$.5401015+.12$

$.5437 ? 00+0 ?$

$.5574 ? 7+0 ?$

-56 6. $2410+42$

- $5751 ? 4$ t

$.5841107+1 ?$

. 5c: $21: 00+1 ? ?$

- $60 ? 36 \cdot 2(4+) 2$

$.5116 .25 \leqslant+02$

- $620541+32$

( $\{904: 7 \div+3)$

$.040006+.3$ 
HN 14 : PCLYACMIAL 201

-.0000000

.0000000

. $1960000-01$

.0000000

$.3942000-31$

- 0000000

- 5647000-É

. $1000000-04$

$.7377000-01$

. $4000000-04$

$.9033000-01$

- $1000000-03$

- $1061800+00$

- ?100000-03

$.121 .3100+00$

- 380000n-03

$.1357600+v 0$

- $600000-03$

$.1455400+00$

- $1050000-02$

- $1 E 26600+00$

- $1500000-02$

- $1751300+0$ ?

- $2340000-02$

- $1369300+00$

- $3320 \mathrm{COO}-\mathrm{C}$ ?

- $1982200+00$

. 4570000-02

- $2088700+00$

- $150000-02$

- 2139301$)+00$

- 3100000-0?

- $2284200+00$

- $1049000-01$

. $2373700+00$

- $1336000-01$

$.2457700+00$

- $1686000-01$

. $2536600+00$

. $2085300-01$

- 2610300+0u

- 2560000-01

- $2579200+00$

- $3112(0)-01$

- $274.32 .00+00$

- 3>43i() ) - I

- $2.002600+00$

. $4477000-01$

. $2857400+00$

$.5303000-01$

- $270>30()+C i)$

- $\therefore 350000-01$

- $2454100+00$

$.7312000-01$

$.27 \rightarrow 6100+011$

APPROXI MATICN PROB:E.A

- $1000000+01$

- 0000000

- $1000003+01$

- c000000

- $1300000+01$

- c000000

- $1000030+01$

- 0000000

- $1000000+01$

- 0300000

- $1000000+01$

- $1000000-04$

- $1000000+01$

- 2000000-04

- $1000000+01$

. 5000000-04

- $1000000+01$

- $1100 \mathrm{CCOO}-03$

- $1000000+01$

- $1900000-03$

- $1000000+01$

- $3200000-03$

- $1000000+01$

- 520CCO0-0.3

- $1000000+01$

- उ०COOC:0-03

- $1000000+01$

- $1190000-02$

- $1000000+01$

- $1720000-02$

- $1000000+01$

- $2430000-02$

- $1000000+01$

- .336000J-0?

- $10 \mathrm{COOUO+01}$

.454000:0-02

- $1000000+01$

-0050000-0?

- $1000000+01$

-792:040-02

- $1000000+01$

- $1024000-01$

- $1000000+01$

- $1307000-01$

- $1000000+01$

- $1641000-01$

- $1000000+01$

-?060000-01

- $1000000+01$

- 25483C00-01

- 1 () ग000+ग1

- 3125000-01

- 1 ก กnocoto1

-3⿻302000-01

- $1000000+01$
- 0000000

- cosiono

- 200civo-01

- 0000000

$.4000000-01$

.0000000

- $5000000-01$

- cc00000

- $8000000-01$

.0000000

- 10000000

- oc00000

- $1200000+00$

- ccono0o

- 14 orjon+oo

- $1000000-04$

- $160000 u+00$

- 2c)幺:00-04

- 1 alou $00+00$

- 3c00000-04

- $2000000+00$

- $000000-04$

- $2200000+00$

- $1100000-03$

- $2400000+00$

- $1900000-03$

- 2600000+00

. $3100000-03$

- $2800000+00$

- $4900000-03$

- 3000000+00

. $7300000-03$

- 32.00000+00

- $1070<00-02$

- $3400000+00$

- $1540000-02$

- 36c0000+00

- 21 प्र0000-0?

- $3800000+00$

- 3010000-02

- $4 \operatorname{con} 000+00$

- $4100000-02$

- $4200 \mathrm{U}: 00+00$

$.54 ; 0<00-0$ ?

$.4400000+00$

- 7260000-02

- 4000000+00

- $947 \mathrm{c000-02}$

- $4800000+00$

- 12231$)(00-01$

- $500110010+00$

- $15(13000-51$

- $5200000+20$

- $1977000-01$

$.5+\infty 0000+00$
.0000000

.00206 .01

$.4000002-03$

$.1200000-04$

$.1500000-02$

- $(1000000-134$

$.3600030-0: 2$

$.220) 000-03$

$.6400000-0$ ?

$.5100000-1.3$

$.1000000-01$

$.1440000-01$

$.16600 C_{0} 0-01$

$.2560000-01$

$.3240000-01$

-4000000-01

$.4340000-01$

$.5760060-01$

$.676001) 0-01$

$.7840000-01$

$.9000000-01$

$.1024000+00$

$.1156000+0 \%$

$.1296000+00$

$.1444000+00$

$.1600000+00$

$.1764000+10$

$.1936000+00$

$.2116000+00$

$.2304000+00$

$.25000(0+01)$

-? $704000+0$ :

- 25 1 - $100+00$ $.13000(1)-i 1$.

.1730 v00-v?

$.2740000-10$

$-41300 n(-1) ?$

- $5430(100-63$

$.9000010-4 ?$

$.106,5000-01$

- $1332000-31$

$.2195000-.11$

$.2700000-01$

$.3277000-11$

$.3930000-11$

$.46 t .6000-01$

$.545,7) \cup 0-(11$

$.624000010-01$

$\left..74^{\prime}\right)+(x)()-.11$

.3514 40 $0-01$

$.97341011-01$

$.1105400+?$.

$.140(11(0)+33$

$.15746,00+07$
$.1758000-61$

$.1250 .2(1)+\ldots)$ 
$.350 .3000-01$

- $3034 ? 00+10$

-933400:)-01

- 30E $340 u+00$

- $1131500+00$

- 3048800+00

- $1296002+01$

- $3125600+(: 0$

- $1477600+00$

$.314900 .3+30$

- $1677700+00$

. $3163900+00$

- $1397500+00$

- $3185600+60$

$.2138100+c 0$

. $3199160+0 \%$

- $2401000+00$

-.3207500+00

- $26.3740 u+C u$

- $.217100+00$

- $2578700+c 0$

- $3221300+30$

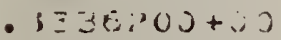

- अ2 $23 \rightarrow 00+J C$

- 370150$)+(0$

- $3 \hat{2} 2.3300+C 0$

- 4n $30 \mathrm{J0} 0+00$

- 3220 âcotu

$.4521200+00$

. $3214700+60$

. $4973700+00$

$.3206500+00$

$.5470100+02$

- $\therefore 196500+u 0$

- $5957000+00$

$.318483 i 0+00$

$.6561(10 u+i) 0$

$.3170500+(1)$

. $7163000+$ UC

. $3154500+10$

$.7807500+6$

- $3136000+30$

- $3473500+00$

- 311600 ist 0 ?

- $9223700+0$

- $3395500+00$

- $10000001+01$

$.3072700+J u$

$.10 .324 .30+31$

- 3048?0)+110

- $1169300+01$

- $3022500+00$

- 126248.$)+31$

$.29951 \mathrm{Co}+\mathrm{Co}$

- $1360490+01$

- 2 SE E.OO +

$.14(.4100+01$

$.2935307+1) i$
- $45 ? 3(0) 3-31$

- $1030000+01$

-5507000-01

. $1030000+1$

. 6 .

- $1000000+01$

$.7776000-31$

- 1 ) coc:3 + 1

- s1s1000-01

- 1)000( J+01

- $1073700+00$

- $1.000000+.1$

- $1252300+04$

- $100000+01$

- $1453300+00$

- 10 oroor $3+01$

- 1 c $8070 u+r u$

- 1 ) $2000+31$

- 14.34y00+00

- $1000003+01$

- $2: 130(0)+00$

- 1 vilucotor

- $2535300+00$

- 1iovo( $0+1$

. :367? $(1)+c)$

- 1u0( 00is)+01

- 1 i 76853$)+0$ ?

- $1000000+01$

. $3707+3)+0 s)$

- $1000000+01$

- $4182130+00$

- $1000000+01$

$.410+300+00$

- $1000000+01$

$.52 .773 \mathrm{Cu}+\mathrm{Ou}$

- $133000 j+01$

- $250470 u+00$

- 1 uvec(cut 1

- $05 \geqslant 0300+00$

- 1 U CUCUJ+ 1

- $733: 000+00$

- $1002000+01$

- $3153700+n 5$

- L') 0000+01

- (30.3y20j+00

- $1306000+31$

- $1000000+01$

- $1000000+01$

- $11: 4$ ن 33.$)+01$

- $1000000+01$

- $1 \dot{c}^{160} \mathrm{cos}+01$

- $1000000+01$

- $133+232+01$

- 1 U000レ?+01

-146,5330+01

- 10 cnocotol

- 1 i1, $310+01$

- 1 a) $0000+01$
. $247+000-01$

-j500u00+oo

- 3034000-01

- 58.10000+00

- 3407000-01

. $00000.10+00$

. $4666000-01$

. $0200000+00$

- 5630)00-01

. $0+0$ cuos+uo

- $6372600-01$

- E600000+0J

- s 26 5000-01

- 58000u்too

- 5र87001-01

- $7000000+00$

- $1176,000+00$

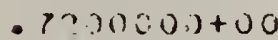

- $135.3100+00$

$.7401000+00$

- 104>1Cu+0u

- teuriuj+ou

- 1 i 270$) 0+00$

- $73: 30(00+00$

- 25?0)0+on

- sconunioton

- 20 ? 1400+00

- $820(j i) 00+00$

. 3541$) 1(10+00$

- 34 (i)(16) Uง+uo

- $351.3000+00$

. 860u.)0u+00

. $4045700+00$

- उ8isu000+00

$.464+000+00$

- $x_{\text {coinonoton }}$

- $5.314400+00$

- $510000+00$

- col ic uotoj

- 94 joñotoo

- उ Es $700+00$

- $\rightarrow$ - $30000+00$

- $7327000+00$

- $9303035+00$

- $8553400+00$

- 1 0ก(ग) +01

- $1000(100+01$

- $10.20000+01$

- $1120160+01$

- $1040300+01$

- $1255320+01$

- $1060000+01$

- $1418520+01$

- 10 s $) 0 u+01$

- $1530-370+01$

- 110 (): $00+01$

- $1771, \mathrm{r}, 0+01$

- $112000 u+01$
$.3136000+01$

$.1756200+60$

$.3304331+3$

$.14 .5111 \cdot 5+($.

$.3600000+00$

$\therefore 1 \div 0023+00$

$.3844036+00$

- $2392300+100$

$.4396,000+02$

- $201+01)+00$

$.4 .35 .0000+0.1$

. $2375025+0.3$

$.402+0) !)+00$

$\therefore 114: 0 ?+1: 3$

$.4903000+3$ ?

$.3432390+17$

$\therefore 134(100+2)$

$.37: 2,205+4 ?$

$.547(1)(1)+1)$

- +1) $\because \because 70+(10)$

$.5776)(0+19$

$.43 .5 .(300+0.1$

- $6(042) 311+11$

$.4745,5,00+1,9$

$.6400000+13$.

. \$120000+0u

- $6722000+00$

$.5513700+00$

$.7056000+00$

$.5927000+00$

. 73.56000+ii

- $3 f \cos (0+00$

$.774100(+0)$.

$.6314706 i+00$

- $810(30 \mathrm{n})+.(\cdot)$

$.72+1406+.10$

$.84(1+000+0)$

$.7786 \cdots 19+1)$

$.8836000+C^{1}$

- $2305,-400+1) ?$

$.9216000+6 J$

- $244740+10$

- 960400$)+00$

$.9411000+10$

$.1000000+31$

- 1$) 0(1) 0.3+1$

$.1040400+01$

- $10 \div 1: 1 u+i 1$

- $1031600+01$

$.112 .5600+01$

$.1124 .(, 1)+21$

- 114, 1020:01

- $11 \cos 40+01$

- $1 ? ; ;, 710+\cup 1$

$.1210000+01$

- 13.71.$)(30+(1$

$.12544(10+01$

$.1404530+01$ 
$.157352(1+01$

- ?300001+i30

- 1683 jक

$.287+100+00$

. $1910640+01$

$.2341100+0 u$

$.193375)+01$

$.2807200+00$

. $2073600+01$

- $2772500+00$

. $2215330+01$

$.2736500+0$.

- $3642^{3} 10+31$

- $2700600+60$

- 2520470+01

- $26 \in 3600+(10)$

- $26,94350+21$

- 24.26000+00

. $2858100+01$

. $25.0370(0+i)$

- 3i) $35360+r i 1$

- $234.1000+130$

- 3ź24180+01

$.25094130+01$

- $34 c^{3} 1(2 \geq 0)+61$

- $2+7010)+2)$

- 2t $2674 i+01$

$\therefore 430100+i 0$

- .364 $1600+01$

$\therefore 389730+00$

$\therefore 4005870+01$

- $234500 u+00$

-42.39820+01

.230 .3100100

$.4543(2 ? 2+01$

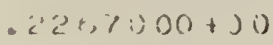

-470)!300 31

$\therefore 2.570)+11$

- j0(25)0+u1

- $11350(0+00$

.3 .337 .250101

$\therefore 14<.300+00$

-56:44,0101

- $2101.200+110$

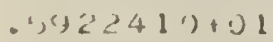

$\therefore 05(3)(1)(1)+1 . j$

- c. $232010+u 1$

- $201, \$ 100+i 3$

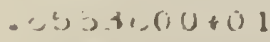

- $1,76, \quad 500+2)$

- 8 . $5(4(3)+1) 1$

- ict3.3.30) $30(1)(3$

. $72.31 ., 50+(1) 1$

: 3, 3.37)+, )

$-10,4,10+31$

- $1052001+3(1)$

. $315,6,111, \ldots 1$

- ir $11,(0)+$,
$.175 .234 J+J 1$

. $1000100+31$

- $15,23410+01$

- $1000(00+01$

-?10n34u+01

- $1100000+01$

$.2297760+01$

- $1000000+01$

- $24838320+01$

- $1000000+01$

$.2702710+01$

- $1000000+01$

. $2131630+01$

- $1000000+01$

-3175300+01

- $1000000+01$

$.3435470+01$

- $100000 J+01$

$.37129 .30+01$

- $1300(100+01$

- $40074 t, 3+01$

- $13 \mathrm{con00+)1}$

-432) $100+01$

- $1000000+01$

- 4 . $523+0+1) 1$

- 1 (ว $6(0) .0+)$.

- 5,01$)+(10+01$

- 1 (1) $10000+1) 1$

- 5.273 $2.40+01$

- $1000000+01$

$.577353 \mathrm{~J}+01$

- $1000000+01$

.t, $191740+01$

- 1 uve $000+01$

- 6) $3.38 .30+i) 1$

- 1 v00000+01

$.7100320+01$

$.1030000+01$

$.7593750+01$

- Iv0u000+01

- 311303$)+71$

- 1300000+01

- sic 171$)+101$

- $1300000+01$

- $\forall 2.183900+131$

- $1300300+01$

- $98405 \pi)+01$

- $1000000+01$

$.104357 b+02$

- $10300(10+01$

- $1115 ; 71102$

- 1 isnousotor

- 11 iscusóst Oc.

-1) 10.0rotol

- 1،( 24 (j) $j+0 ?$

- 11,30000+o1

- 1 i jei: / sto :

- Iucuevotul
- $19733201+01$

- $11+1) \cup 0(1) 1$

$.2154970+01$

. $110(0) 0+01$

- ?4 $43+2)+02$

- 1 esulicutol

- 2540550+01

- $1203000+01$

- 24 -

- $12.2000+01$

- $3297307+21$

- $1247000+01$

- 3633 3.?1)+31

- $1200: 100+01$

- $4001500+j 1$

- 1 ?iji)00u+u 1

- 4 20150+01

- $1300000+01$

- $4826310+01$

- $132(1) 00+01$

- 523 $3350+01$

- $13+0(i) 0+01$

$.573(-340+1) 1$

- $1313000+01$

- $63.3, ? 0+01$

- 1 iif $: 0(3)+0) 1$

- $(7) 1: 761)+01$

- 1 a $) 6.00+01$

- 75? (3):50+01

- $1420000+01$

- $31: 3420+01$

- $1440000+01$

- $341 \cdot 100+01$

- $14600010+01$

$\therefore 535300+01$

$.14 .31000+01$

- $1050 \times 22+02$

- $15015000+01$

. 11.301$) 6.3+02$

- $1: 300.30+01$

- $1233 \% 32)+02$

- $1540,000+01$

- $13.3,30.3+0 ?$

- Ijocionoto 1

$.1441 ? 77+102$

- $15330(100+01$

- 15 5. 57 isoto?

- $1520000+01$

$-1677722+02$

- 1 is. $0100+01$

. $19.7754+02$

- $10+02.00+(1)$

- $1345+4.3+0 ?$

- 1 (1, 0) $12+31$

-2. $\div 41$ istu?

- $11 ; 00,0.101$

- 24 i $307+02$

$.1700,1)+01$

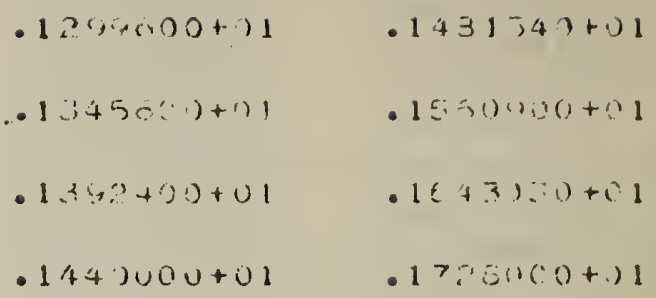


- 3352160+?1 - $1770300+1)($ ? $.4752130+1) 1$ - $1730100+60$ $\therefore 1$ uf $3(0)+c 1$ - 15:972 J+0J - . $5551.30+j 1$ - $1049 i 00+00$ - $1003876+02$ - $1509300+00$ - $1049760+0$ ? - $1570200+00$ . $1097199+02$ - $1531000+00$ - $1140227+02$ - $1492100+00$ - $1196\{183+02$ - $1453500+00$ - $1249168+0$ ? - $1+1 j 4 \mathrm{CO}+0 \mathrm{u}$ - 130 37 $10+0$ ? .1377 c $00+00$ - $1352455+n$ ? - $134.3 \because 30+c 0$ - $1416,469+0 \%$. - $1303200+31$ $.1475789+02$ - $12 \in \in 700+30$ - $15.56454+0 ?$ - $1230600+00$ $.1600000+02$ $.1195000+00$ - $1664960+02$

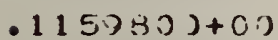
$.1731 .391+02$ - $1125 i j 00+0 j$ . $1.300 .314+0 ?$ . $1090800+i) 0$ . $1371774+02$ - $1057100+00$ - 1944:310+1? - $10 ? 3.301+v 1)$ - $: 01-2,63+3 a$ - ta $10000-01$ -? ?97: $74+0 ?$ -758:300j-01 - $217578 \Omega+02$ - $2271000-91$ - $2253531+0 ?$ - $4953000-01$ -?34?560+0? - $1651000-01$ - $2428913+0$ ? - $: 350000-01$ . $2517631+02$ - अ $53000-11$ - $200375 \mathrm{c}+02$ .776 ? 200.)-?1
- $1+1+37+0$ - $1300003+01$ - 1 jo j3i.6+i): - 100000$)+01$ - 1344 [.. $7+v=$ - $100(i)(2)+31$ . $1 \vdots \succeq 742+1$ : - $16: 2000+01$ - $1780899+0 \%$ - $1000000+01$ - $1489568+02^{2}$ - $1.00000+01$ - $1396 ; 03+02$ - $1000 \mathrm{cc} 0+01$ - $2109061+02$ - $1000000+01$ - 2220? $3+0$ ? - 10 co:ic J +0 1 - $2=43 \% 13+0:^{2}$ - 1u00u00+01 - $2478,50+0$

- 1 ivclouto?

- 2e. $07193+22$

- 1000c0u+ul - $274+75+48+0 z$

- $1000600+01$ - $2832547+0$.

- $10000: 3+01$

- 3i) $431<3+0:$

- $100 u c 00+01$

- 320U0Uu+Oद:

- $1: 20000)+$ ? !

- $3=401232+02$

- 1 i) $00003+01$

- $35=3053+0 ?$

- 1$) 6(10)+01$

- $370907.7+02$

- $1000000+01$

- 3\%9.32.3 $3+0 ?$

- 1$) 30(1)+01$ - 4 ) $84101+02$ - $1000000+01$ -4342322+0\% - $1,3000(1)+01$ - $44601 i t+02$ - $13 j 0$ J) -470135j+0? - (1) 3)(jo)+01 - 4923597+0\% - $1000000+01$ $.5153632+32$ - $100000 \mathrm{i}+01$ - 5.39:21.8j+0í - 10 j $)(10+01$ - $5039493+02$ - 130100101 - $5835743+0$ ? - $133(10(1)+01$
- $24157 \div 7+02$ - : $7 \therefore 910)+01$ - $25-3 \cdot 23 u+02$ - 1 (a) $0(10)+31$ $\therefore ? 7.2703+02$ - $173.2001+J 1$ . $257218 z+0 \%$ - 17 tinéoutol - $31306,80+02$ - 180 cocoto 1

- $3401: 2 z+02$ - $1320000+01$ - $3634363+02$ - 1840 U0+ J1 - 38xCo $72+0 ?$ - 1 36:0:00+01 $.41407 .77+32$ - 1 . 3300001+C 1 $.441 \div 1 \div .7+02$ - $1300600+01$ - it 71)4:58 $43+02$ - 1 :3risunto 1 $.50006 .50+02$ - $1440000+01$ - 53.31 J $1+02$ - 1 >60, out. 1 - $5(1,351+02$ - 1.):30.) jot 01 - (c) $2547 x+1+2$ . $2000 \mathrm{a} ; 10+01$ - 64 Cnouj+ 32 $.2020000+01$ . $6743729+02$ . 2040$) 00+.31$ $.7207 .40+02$ - cononcutol - $7041 ; 3 b+0 ?$ - 30130300+)1 - $8 \mathrm{c}+8 \mathrm{~g} 0 .+2+02$ - 21 (1)inus 1 - $357 \therefore 612+0 ?$ - $2120000+01$ $.9073 i: 2+02$ - $21+0.300+01$ - $4004 \div 74+32$ - 2100000+J1 - 1$) 15: 00+03$

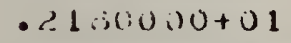
- $107: 34+403$ - $2200100+31$ - $11.3744+03$ - $2220000+01$ $.1147) 65+0.3$ - $2.2400 .30+31$

- $1253245+03$ - $220000 u+31$ - $1 \pm 32443+0.3$ - $23(3)(1) 11+31$

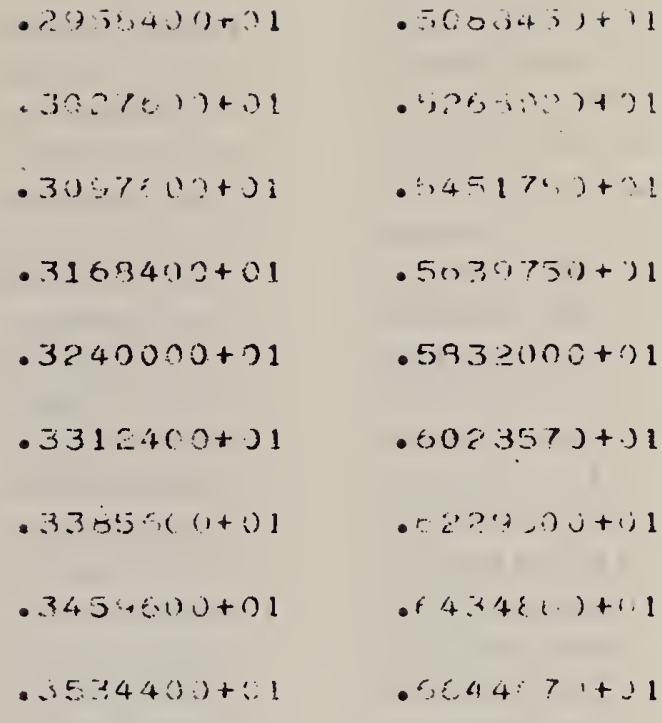

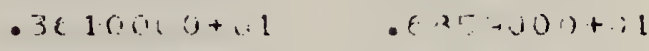

$.308(14(10+01 \quad .70774+11+.11$

$.376 . .6(10+112 \quad .7 .40 .1 ; 50+11$

$.3841(0 u+01 \quad .73,254 u+11$

$.3 .03403+01 \quad .77(32=03+11$

$.4000000+01 \quad .2300000+1$

$.4080+1,0+01 \quad .924 ?+1: 1+11$

$.41516(1)+01 \quad$. $844(20)+5(3+11$

$.42 .4 .3500+01 \quad .874162 .01$

$.4325400+01 \quad .3034310+11$

$.4410303+01 \quad$. (12, 1, 1) $13+1$

$.44544(10)+012.9328131+11$

$.4574500+01 \quad .48003 i 6+11$

$.46(5 x)+131 \quad .10) 777 .+13$

$.475 ? 4001+01$. $10.3619: 3+1 ?$

$.4340000+31 \quad \cdot 1364300+?$

$.492340 !+01 \quad .109+105+02$

$.5017000+11 \quad .112242+13$

$.5107601+31 \quad .112,31+3+12$

$.5193400+31 \quad .11 .3 \% 35+23$ 
$.2702336+02$ . $7476000-01$ . $2798410+02$ . $7196000-01$ - $2397023+02$ - $6921000-01$ . $2998220+02$ . 5651000-01 . $310 ? 044+.22$ . $6337300-01$ - $3208543+02$ . $0123000-01$ $.3317760+0$ ? . $5874000-01$ . $3429742+02$ . 5626000-01 . $3544535+0$ ? . $5383000-31$ - 3652186+02 $.5145000-01$ - $3792742+02$ . 4913000-01 - 3906250+Ja - 1685000-01 . $4032758+02$ . 4463000-01 . $4162314+02$ . $4247000-01$ - +294967+02 - 4035000-01 - $430766+02$ - $3329000-01$ - $456 \% 760+0 ?$ - $3627000-01$ . $+711 \mathrm{~kg}+3$ ? . $3431000-01$ - 4 357532+0? . 3240001$)-01$ . $5006412+c 2$ . $3054000-01$ . $5158087+0$ ? - 28372000-01 - $5.314410+0$ ? - zE 36000-01 . $5473632+02$ - .2524:000-01 - $5636406+22$ $.235 ? 0) 2-31$ .5802745+22 - $2195000-01$ - $5972817+0 ?$ - .20 37000-01 - $6146: 362+J)$ - 1 !: $34000-01$ - 1324001+0? $.1735000-J 1$ - $505300+0 ?$ - $15 \rightarrow 10 \mathrm{CO}-01$
- क $1613: 7+13$ - $10000 u v+.1$ - $5430.343+0 ?$ - $1000000+01$ - (37? 1 i) $93+0 z$ - $1000000+01$ - 7'J15334+02 - $1000000+01$ - $7320825+02$ - $1000000+01$

- $7630332+02$

- $1000000+01$

. $7962624+02$ $.1000000+01$ . $3299576+0_{4}$ . $1000000+01$ - $8640065+02$ - $1000000+01$ - $9008578+02$ - $1000000+01$ . $9381200+02$ - $1000000+01$ $.9765625+02$ . $1000000+01$ - $1010255+0.3$ - $1000000+01$ - $1057228+03$ - $1000000+01$ - $1059512+0.3$ - $1300000+01$ - $1143133+03$ - $1000000+01$ - $118 \times 313 \%+03$ - $1000000+01$ - $1234544+03$ - $1000000+01$ - $128: 339+0.3$ - $1000000+01$ - $1331705+0.3$ . $1000000+31$ - 1 18:523+0? - $1000000+01$ - $1434391+03$ - $1000000+01$

- 14 ट8टट3+03

- $1000000+01$

- $154+375+03$

- $1000000+01$

- $160156,8+03$

- $1000000+01$

- 1 (s) $(44 \ldots+3$

- $1000000+01$

$.1721037+03$

$.10000010+01$

$.178 .2337+0.3$

- $10000010+01$

- $1.347531+03$

- $100(1000+1) 1$
- $1404133+03$ - $23100000+01$ - $143 J 35 y+03$ - $23200 \ddot{U} 0+01$ - $1554 \therefore 7++03$ - $2340000+01$ $.1641735+03$ . $2360000+01$

- $1727715+03$ - 233 i ( )

- $1 \geq 17447+03$ . $2400000+01$

- $1911030+03$ - $2420000+01$ - $2033594+03$ $.2440300+01$ $.2110 \cong 75+03$ - $2460000+01$ - $2216: 04+03$ - $2430000+21$ - $2326538+03$ - $25100100+1$ $.24+1+06+3.3$ - 25200 $00+01$ - $2300 \div 03+03$ . $2540000+01$ - $2035359+13$ - 25ev000+01 - $2814750+03$ - 25 Bu $000+01$ - 29492y5+0 3 - 26$)(100+01$ - $303 y 158+03$ - $2620000+01$ - $32.34504+03$ . $2640000+31$ - $3335506+03$ - $2060000+01$ . $3542337+0.3$ - $2680000+01$ . $3705175+03$ - $2700000+01$ $.3874205+03$ . $2720000+01$ $.4049612+0.3$ - $27+0000+01$

. $42.31388+03$ . $276(100)+01$ . 44?.) 3. $8+03$ - $27.40000+01$ - $461003 ?+0.3$ - $2800000+01$ . 4 r.318.003+03 - $23 . ? 1000+01$ - $50: 3151+0.3$ - $2840000+01$ - 5.34.0. $393+03$ - $2360000+01$
$.5240000+01$ - $121 \in ? 00+0 ?$

$.5382400+01$

-1243717+0?

$.5475600+01$

- $12312 ?)+02$

$.5559000+01$

$.1314+26+0 ?$

$.5664400+01$

- $13481 ? 7+0 ?$

$.5760000+01$

- $1382400+0$ ?

$.5856400+01$

$.1417249+02$

$.5053603+01$

$.14526 .73+12$

$.6051600+01$

$.14886744+72$

$.6150+00+01$

- $1 ; 252 \div c+9 ?$

- $0250000+01$

$.1368500+0 ?$

- $63504(10+(1) 1$

$.16: 00 \geq 01+0 \geq$

$.6451600+31$

$.1638700+02$

. $6553600+01$

. $16777.2+02$

$.6 t .50400+01$

$.1717 .3 \% 1+32$

$.0760000+01$

$.1757000+1) ?$

- $f 86,440 u+01$

$.17 \div 347.3+19$

- 606.90$)+(: 1$

- $1436774+12$

$.7075600+01$

- $1362110+2 ?$

$.7192400+01$

- $14.245 .3+1,3$

.72.) 00(0)+01$

$.196 .9300+12$

$.739: 4400+01$

- ?.ก1236.5+1?

$.7507600+01$

$.7517000+01$

$.7728400+01$

$.7443000+01$

- $7352: 4(1)+1$

. $80 t 5000+01$

- $31750.00+01$ - $28+2 \div 77+u=$

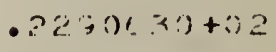

$.20 \div 70<2+3$

$.2102452+? ?$

. $148405+0 ?$

. $21952(1)+02^{3}$ $.2334386+12$ 
$.0690586+02$

- $1452000-01$

- $6879707+0 ?$

. $1316000-31$

- $7072810+0:$.

$.1185000-01$

$.7267550+0 ?$

$.1059000-01$

$.7471182+02$

- $7360000-0 ?$

. $70.75553+02$

. $3170000-02$

. $7886150+02$

- 7230000-0?

. $3100000+02$

.5920000-02

. $3318170+02$

- $4850000-0 ?$

. $8540717+02$

- $3820000-02$

- $8767701+02$

- 2830000-02

- $3999179+02$

- $1870000-02$

- $7235210+02$

- 3500000-03

- $\because 4 ? 5854+02$

. $7000000-04$

$.9721171+02$

- $7800000-03$

- $9971221+02$

- .16 uc000-02

. $10226,06+03$

- . 3380000-02

- $1048576+03$

- $\therefore 130000-02$

- $1075037+0.3$

-3350000-n2

. $11015(30+1) 3$

. $4530000-02$

$.1129459+0 j$

. $5190000-02$

$.11 ; 7432+03$

- jis 20000-0.?

- $1135421+0.3$

- .6.420000-0.

- $1214433+03$

- (r) $80000-6:$

- $1244474+0.3$

-.7530000-0?

. $12.74551+03$

-.8040000-02

. $1305169+03$

-.35.30000-02

. $133 \epsilon 33 c+03$

-.39:0N00-02

- $136.8058+03$

-.34.30100-0?
- $1513507+03$

- $1000003+01$

- $13831=5 \%+0$.

- $10000 n)+01$

. $\therefore 051113+0 \div$

- $1000000+01$

- $2122325+03$

- $1000300+01$

- $2195528+03$

- $1000000+01$

- $2272263+0.3$

- $1000000+01$

- $2350073+03$

- $1000000+01$

- ?43:200u+0.3

- $1000000+01$

. $2512 \mathrm{c3} 37+0 \mathrm{~s}$

- $1000000+01$

- $2596373+0 z$

- 1000001$)+01$

- $2682910+03$

- $1000(0)+01$

- $2771747+03$

- $1000000+01$

. $2862315+03$

- $1000000+01$

- $2555467+03$

- $1000000+01$

- $30 \leqslant 2448+03$

- $1030000+01$

$.3151) 906+03$

- $10 \operatorname{co0} 00+01$

. $3251: 388+0.3$

- $1000000+01$

- $3355443+03$

- $1000000+01$

$.3451020+03$

- $1000000+01$

- $3570467+03$

- $1000000+01$

- $3582036+03$

- $1000000+01$

$.3796 .375+0 \%$

- $1000000+01$

-3y13537+ J3

- $10($ i) (1) $)+01$

- $40.33578+03$

- 10 CUNU1) +01

- $4156544+03$

- 100 unotol

. $4282430+03$

- $1000000+01$

. $441147 z+03$

- $1000010+01$

$.4 .54 .3542+0.3$

- 1.) $00000+01$

- 46.78758+03

- 1$) 23000+01$
$.5472631+03$

- $28.40000+01$

$.570) .304+0.3$

- 24uru00+31

- $554+233+0.5$

- $2900000+01$

- $E 198650+03$

- $2940000+31$

. $6457791+03$

- 2900000+01

. $07.55998+03$

. $2930000+01$

. $7003217+03$

-3000000+01

- $7200000+03$

- 3020000+01

. $7530504+03$

.3040000+01

- $7892+89+03$

$.3160100+01$

- $520+724+03$

- 5030000+01

- $8535781+03$

- $3100000+01$

- $2875037+03$

- 312010$) 0+01$

- $522+176+0.3$

- $3140000+11$

- $958403 E+0.3$

- $3160000+31$

- $5956.962+03$

- $3130000+01$

- $1034100+04$

-j:20(15)00+01

- $107.374 i 2+04$

- 32201000+01

- $111+i+2+04$

- $3240000+01$

- $11503331+04$

- $3250(00+01$

- $1200344+04$

- 32 $36000+01$

- $124 \vdots 211+04$

- $\therefore 3$ 3iveivutol

- $123140 j 3+04$

- $3 \because 00001+01$

$.01=3014 \xi 3+04$

$\therefore 340000+01$

- $13882805+04$

.33006uj+01

- $14 j 3 \cdot 17+04$

- 3360000+01

- $1491077+04$

- $3400000+01$

- $1544: 304+04$

- $34: 0000+01$

- 161$) 0135+04$

. $3440000+01$
. $3294.10+01$

- ?33.3743+? ?

$.3411)(10)+01$

$.24 .58 .00+j 2^{\prime}$

$.8526400+01$

$.24 .9700+0.8$

$.83643600+21$

$.2541213+6 ?$

- $83761600+01$

$.2593424+02$

- $88380400+01$

$.2646 \cdot 359+02$

$.9000000+01$

. $730000+02$

$.9120400+01$

- $2754.361+0 ?$

$.92+1<00+01$

- $28109440+19$

- $936: 1600+01$

- $2 \cdot 65 ? 0 ?+1 ?$

- $94630410+.1$

- $29 ? 1311+32$

$.9610020+01$

$.297 .9100+1 ?$

$.9734400+01$

- $30371 ? 3+.2 ?$

$.985 .7600+01$

$.30 .35014+102$

$.9785600+01$

$.3155450+0 ?$

-1) $11240+0 ?$

- $3215743+0 ?$

$.1024000+02$

. 3276-ivoto?

$.1035 .343+0 ?$

- $33.48625+0 ?$

$.1045700+02$

$.3401222+10 ?$

$.106 ? 760+0 ?$

- $34645483+0 ?$

$.1075830+0$.

$.352375 \%+.19$

$.1030000+1) .2$

$.35937)(1+3)^{\prime}$

$.11(1224:)+0$

- 3i) $34437+03$

$.1115563+0 ?$

- $\$ 7: 25477+0 ?$

- $11 \div 24.760+0 ?$

$.379 .3706+12$

$.1142440+02$

- 3ose $1447+0$ ?

$.1156000+0 ?$

$.303 .3049 n+72$

$.110,36,40+03$

- $4000165+0 ?$

$.118:-350+02$

- $407075,3+0 ?$ 
- $1400341+03$

-.3840000-02

$.1433192+03$

-.1023000-01

- 1460 E $18+i .3$

- $1059000-01$

. $15006<5+03$

-. $1094000-01$

. $1535220+0.3$

-.1126000-01 $.1570410+03$

$-.1155000-01$ - $1006201+03$

$-.1183000-01$ $.1642601+0.3$

-.1209000-31 $.1679616+C .3$

-.1233000-01 $.1717253+03$

-.1255000-01 $.1755519+1.3$

- $1275000-01$ . $1794421+03$

- $129.3000-01$ - $1833965+.3$

-.1310000-01 - $1374161+93$

- $1325000-01$ . $15.15 J 1.3+33$

- $1.3 .38000-01$ - 1956531$)+03$

-.135000 $2-01$ - $19987717+03$

-. $1360000-31$ - $2041584+C .3$

-. $136.9000-01$ - $\therefore 085136+0.3$

$-.1376000-01$ - $2124.381+03$

- $1332000-01$ $.2174 .327+0.3$

-. $1337000-01$ . $3214981+1) 3$

- $1330000-01$ . 2266351$)+i 3$

- $1352000-01$ . $2313441+(13$

- $133000-01$ - $2311262+03$

- 13 y $3000-01$ . $240.322+0.3$

- $136: 3000-01$ - $2454126+c .3$

-.13r 1000-i) 1 $\therefore 50519.2+0.3$

- $133(, 1) 00-01$

$.25,01) \cup u+43$
$.4317173+03$ . $1000000+01$ . $4958.345+0.3$ - $1.00000+01$ $.510 .33 .10+03$ - 1 (1) $100 j+1)\}$ - 52521.37+u. - $1300000+01$ $.5403975+03$ - $1030000+01$ $.5559251+0.3$ $.1000000+01$ $.5718077+0.3$ - $1000000+01$ - $5833512+0.3$ - $1000000+01$ - $0046013+03$ - $100000 i)+01$ -5216.4.36+0.3 - $1.100000+01$ - $6350083+3$ - 100 vounto1 - $6567581+07$ - $1000000+01$ $.6743990+03$ - $100000 j+01$ - $+43+395+3$ - $1000000+01$

$.7123 .349+03$ - 1 1 ocog)+01 $.7317421+07$ - 100000$)+01$ $.751: 177+0.3$ - $1000000+01$

. $7717187+0.5$

- 11000000+ 1

- $7923517+03$ - $1000000+01$ - $31.3+237+10.3$ - $1300000+01$ - $8.349417+0.3$ - $1000 \mathrm{v00}+01$ - $33569120+0.3$ - $1300000+01$ - $137934.37+0.3$ - 11) OC rivotol - 90234?0+0.2 - 1 ) $0 \cup(10+01$ - $325614 \%+0 \div$

- 1.)0000u+ul $.4494+, 47+0 . ?$

- 1)000uot)1

- $973+131+03$

- 1 (j) $)(i 1)+01$

-. $9830547+0: 3$

- 1 u JuU(i) +01

- 1 i) $2400.0+04$
$.1657107+04$

- 346r:000+01

. $1715760+04$

- 34 (i) $000+01$

- $17 ? 01$ : $3+04$

- $3500(100+21$

- 1 E.332C6+04

. $3520000+01$

- $1902190+04$

- $3540000+01$

- $1967975+04$

-.356) $30+01$

- $20.35635+04$

- 35.3000u+01

- $2105223+04$

- $3600(1) 1)+01$

- $217673: 34$

- 36.20000+1)1

- 22.19357+ 14

- 36:0! $00+01$

- $2325(112+04$

- $3680000+01$

- $240 \div 735+04$

- 30+30000+01

- $24.30030+04$

- $37060.10+01$

- $2565726+04$

- $3720000+01$

- $2090072+04$

- $3740000+01$

- $2736715+04$

- $376,0000+01$

- $2 \mathrm{a} 22^{4} 5707+04$

- $373(1) 301+31$

- $2917097+04$

- $3: 3(1 ; 1) \mathrm{CO}+01$

- $501.0350+54$

- รम?2) $3(1+51$

- $3107 \geq 7 y+04$

- 3340000+01

- $3206,1760+04$

- $336000+01$

- ;31) $7.8 . j+04$

- $3530600+01$

- $3411: 353+04$

- 3500(:00+01

- $351.3744^{\circ}+04$

- s.joc $1100+01$

- $3628410+04$

- 3540000+1)1

$.37+0411+04$

- $3400000+01$

- $3956303+04$

- 3c, 30)0u+0 1

- 34; 74i, 45+04

- 4 uno000+01

- $40.300(0)+04$
$.1197100+13 ?$

. $41+21 ? 4+12$

. $1211040+0 ?$

$.4214+1(3+2) ?$

$.122500 j+0 \%$

-42 $4759.1+32$

- $12300+0+0 ?$

$.436,1+21+.12$

- $1253160+02$

$.4436186+02$

$.1267360+0 ?$

.+511 402. + 2

$.12,16,4)+?$

$.45833271+02$

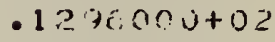

$.4 i .8 \leq 00+6$

$.1310440+3 ?$

$.474 .371 .7+11 ?$

$.1324760+0 ?$

- $43 c^{2} ? 3 ! 4+40 ?$

$.1339500+0.2$

- 4ci) $2(3(1)+\ldots$

$.1354 .240+02$

$.4+3.3603+13$

$.136 .4000+0 \%$

-5i) (5.71i)+ij?

$.138 .3340+0 ?$

- $1373763+19 ?$

$.141 .3760+08$

$.14 \Omega .3540+0 ?$

$.1444(1) 0+.0 ?$

$.145024)+0 ?$

- $1474560+i 3$

$.14630 ; 0+02$

$.1505440+0 ?$

$.1521000+02$

$.153(1)+0+0 c$

- $195: 3,0+6: 2$

- $13 \operatorname{ses} 160+02$

$.15 \sin 04(u+1) 2$

$.1000005+0$,
-5147 $540+1$

$.523135 .2+12$

$.531 \cdot 573 \cdot 3+3 ?$

$.5401: 1(j+3 ?$

- $3+57 \div(0+1) ?$

.$: 157+207+32$

- 565:? 31$)+0 ?$

- j7 $72+1+1 ?$

-jos $1107+1) ?$

- วะร1ำ

- $t u: 3 E \cdot ? \cdot i+(1.2$

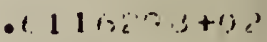
- $(20)+11+1): ?$ - $309+7 a+0)$ $.640(i v()+1) ?$ 
$15: 6 F N=1.41$ $S$

$.1473690+21$ - $1000000-j 1$ - $1113: 7)+(11$ - $400000.13-31$ - -55050$)+3$ - $3000000-01$ - t) $7355 \mathrm{co}+00$ - $1600000+00$ . $5413700+00$ - $2500300+00$ - $4423700+00$ . $3600000+i 0$ $.365 ? 600+00$ $.4000000+00$ - $3043400+100$ $.6400000+00$ $.2554500+C 0$ - $3100000+c 0$ - 2157300+00 - $1003000+01$ - $1027550+01$ - $1000000-01$ - $3843200+00$ . $4000000-01$ . $7300100+00$ $.9000000-01$ . $5984700+40$ - $1600000+00$ $.4927700+00$ . $2500000+00$ - $4084600+00$ $.3600000+00$ $.3408300+00$ - $4300000+00$ $.2862500+00$ - $6400000+00$ - $241650.0+00$ - $3100 j 00+00$ - $2050500+00$ . $1000000+01$ - $t .926100+00$ - $1000000-01$ - $552 \rightarrow 600+00$ - 4000000-01 .5775000+00 - 3000000-01 . 43 t $900+00$ - $1600000+00$ - 4?2̇2100+1) - $2500000+00$

- $1577900+00$

- $3500000+30$ . $3037200+10$ . $4300000+00$ $.2532100+100$

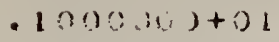

- 1 3) $) \cup(1, j-01$

- $13000(1)+01$

- 1 sc(500s-0 1

- $1000000+01$

- $1000000-01$

- $1000000+01$

- $1000000-01$

- $1003000+01$

- $1000000-01$

- 1 ( ) guv jo 1

- $1000000-01$

- $1000000+01$

- $1000000-01$

- $1000000+01$

- $1000000-01$

- $1000000+01$

- $1000000-01$

- 1.)00000+01

- $1000000-01$

$.1000000+01$

-40c0000-01

- 1.$) 00000+01$

-4000000-01

- $1000000+01$

-4000003-01

- $1000000+01$

-4000000-01

- $1000000+01$

-400000)-01

- 1.)00000+21

-4000000-01

- 1000000+01

. $40 \mathrm{CCOOU}-.71$

- $1000000+01$

-41) CDOLJ-01

- $1300000+01$

- 4000000-01

- $1100000+01$

- 4000000-01

- 1 )

- 9cco000-01

- $1000030+01$

-9000010-01

- $1000000+01$

- 9 गc C八o-01

- $10000 u v+01$

- 9 CCD000-01

- $1000000+01$

- 9c00000-01

- $1000(i c 0+01$

- 9 ccojos-) 1

- $1030000+01$

- $3060032-11$

- $1000000+01$
$.1)(1,04+0.7$

- $1: 3(10.9+3)$

(1)) () $20,-1$

- $20(19) 916+00$

$-10 n+(1)+03$

- 2$) 000(2-31$

$.3000 \cos 0+00$

- $1000.30+0 \%$

- -30$) 39-3$

$.40001100+00$

$.1000000+00$

$.4000000-31$

$.500(1000+00$

- $1000 n 00+60$

$.5000000-11$

$.000(90+00$

$.10000(10+0,1)$

- 70 scuvotuo

$.100000 .0+00$

$.8000030+00$

$.1000000+00$

- $9020000+00$

$.1000000+00$

. $1000400+01$

$.10000(10+00$

$.1000000+00$

$.2000000+02$

- $2000000+00$

$.2000000+00$

$.3000 \mathrm{COO}+00$

$.2000000+00$

$.4001000+00$

$.2000000+00$

$.5006900+00$

$.2000000+00$

$.500000 J+00$

$.2000000+00$

$.7000000+00$

$.2000000+00$

$.3002 .00+00$

$.2000006 j+60$

- cuvujo+oo

$.2000000+c 0$

$.1000000+01$

$.2000000+.00$

$-1000(0)+00$

- $2010(101)+00$

$.3000000+00$

- 4600000+00

$.5000000+00$

. $5000000+00$

$.76) 0000+00$

- is $\cos (100+00$
$.3006000+00$

$.3000000+C 0$

$.3000000+00$

$.3000000+00$

$.3000000+00$

$.3000000+02$

$.3000000+00$

$.300 .0(00+0)$
- $500000-01$

$.7000000-0,2$

$.3000000-11$

$.000000-21$

$.106,100+00$

$.2000000-31$

$.4000000-01$

$.6000000-01$

$.80000100-01$

$.1000000+70$

$.1200000+12$

$.1400000+(1)$

$.15000(0+1)$

- 1 (3) $0100+(2)$

- 50$) 3(20+10$

.3000 .300 .111

- 6000$)(0-0.1$

$.9000000-01$

- $1203600+30$

$.1500070+00$

$.1800000+12$

$. ? 100000+00$

$.2400,00+0)$ 
$\therefore 4000(0)+1)$

- .2. $1000+i 0$

- 3100000+.)

- 1 1531500+1)

- $1030003+31$

- $44350<0+00$

- (1) (00ग-) 1

. $4527500+00$

- $+700000-01$

- $+2.74700+0)$

- ग0(ग00-01

- 3863400+00

- $1000000+3$

- $411(10+0)$

. $2500003+00$

$.2974400+c 0$

- $3600000+00$

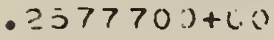

- hacooono

$-22 \pi 7(0+0)$

- $(4,100(0)+01)$

- $19234(0)+0: 1$

-3110000+0j

- $168.1500+11$

- 1(0) $) 00)+01$

. $251750.0+i i 1)$

$.10: 000:)-01$

$\therefore 303600+011$

- $+000000-01$

- 303 $13 \mathrm{COu}+3$

-100000j-ul

$\therefore 75,5000+00$

- $1200000+00$

. ?578700+0)

-jo0)( ) + 0

- $232(2=00+i)$

- $: E, 1) ? 000+(1)$

- ? J70 $700+00$

- 4:1003is J +

- $1327200+00$

- $0436000+60$

- $150+i 300+0 ?$

- 31)0000+(1)

- $1403500+($,

- 1$) \cup i) 00+01$

- $1(104500+0$.

$.1000000-31$

- $148,0700+00$

-400:5000-01

$.1737700+130$

- j000000-01

- $1913200+00$

- $1600000+00$

$.1778 .25)+(1)$

- $55: 0000+($.

- 16,7920 J+i) J

- $60000(j+j 0$

- $154320(1)+$.
. (3.30?)-0!

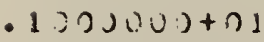

- 9 (3000)- 31

- $103(j 000+i) 1$

- 9 ง $0(\%)-01$

- 11):000 J+01

- 1 ; jo?0s+00

- $1.300000+01$

- 1 to $0(0)+0)$

- 10000u 101

- $18.00: 3)+01$

- $1000003+101$

- $1: 3(3000+0:)$

- $1020(100)+01$

- $1600000+0.9$

- 1 u $20000+1$

- $16.0(i 0)+0)$

- $1003000+31$

- $1600(0)+00$

- $1200(0)+01$

- 1 (.) $3000+00$

- $1 \vdots 30000+51$

- $16,00(i) 0+00$

- 1$) 30 \mathrm{0} 0+3$

- $1800000+00$

- $100.0(1)+01$

- ?500500+ui

- $13 \cos (0) 01$

- $2 \approx(0)(1)+0)$

- 1 (1) $) 1(100+01$

- $25000(0+0)$

- $1000000+01$

- $25 \mathrm{col}(\mathrm{i} i j)+0 \mathrm{c}$

- 1 i) $0 \mathrm{~J}(\mathrm{i}(\mathrm{i})+01$

- $2560(0)+0)$

- $1000(0)+01$

- 2500i(i)+1)

- $1000(0)+1) 1$

- $25 \mathrm{~s}(\mathrm{~s})(\mathrm{s}+0)$

- $1000000+01$

- $25(1)(1) 1+0)$

- 1000000+31

- $: 500400+01$

- $1000000+01$

- 250000$)+(0)$

- $1000(0)+01$

- 360000$)+0)$

- 11$) 0(3) 00+1) 1$

-3e.000:30+.)

- $13(00 ?)+31$

- scovouotou

- $1300000+31$

- $36 \mathrm{CDO} \cup 0+00$

- $130000 \%+01$

- 36000cotoj

- 1.) 2 ) coj +01

- 38 $8000 \mathrm{~J}+0 \%$

- 1 úं
- $\cos (0) 00+03$

$.201,10+00$

- 2? ?):3.? $1+31$

- $10 u(6) 0+01$

. . . $0.1+911+6$

$.31) 30330+(30$

$.100(\ldots, 00+0 i$

$.+00 x-5+0$,

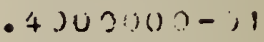

$.2000 .00+00$

$.4000000+6.1$

. 5000$) 00-: 1$

- $30(01) 00+00$

$.400(20)+(3)$

$.1200(0 \%)+u)$

$.400(000+00$

$.4000000+(0)$

- 16 U D.3. $3+10$

$.5000000+00$

$.400 i(i)+3$

$-20(16201+0)$

$.0001960+00$

$.4000000+00$

$.70)(21010+00$

- a jouc: $0+1.3$

$.020(1,00+1)$

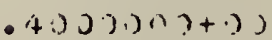

$-\Rightarrow \cos n o+00$

$.40003(3)+$.

-1.)(a) $(0)+3$

$.400(0) i \cdots+i$,

- $10.00010+50$

$.5000001+19$

$.20(10.000+00$

$.500 \div 000+0$ ?

- $300(100)+c 0$

$.50000(0+3 i)$

$.4000(1) 00+00$

$.500003 n+0$.

- $50(j 0 u v 0+00$

- $5000(, 1) 0+16$

$.2000000+00$

$.70(1)(100+00$

$.300(000+00$

- $\rightarrow \cos (1) 00+00$

- 1 cosucotot 1

$.1000000+00$

$-20(\cdot(4) 0) 0+00$

$.3010000+00$

$.40010) 0+00$

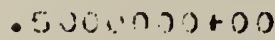

$.6,0010000+00$

$.701() 013+1)$.
$.3000(10)+0$

$.500(.000)+(00)^{\circ}$

$.5000 v()+$.

$.5000000+00$

$.50000(10+0.0$

- $600(1(1) 0+C)$

$.8 .000000+011$

$.600 .11302+0.2$

$.6000000+00$

$.6 .003630+60$

$.600(1)(1) 1+(3)$

- irocosonta.
$-2400(1) 3+(1)$

-? 30$)(10+00$

$\therefore 39000.2+1.2$

- 3is) $000+\cdots$

. 4$)(10) 0 n+6 ?$

- $3.000 .300-01$

$.10) 3003+\because 3$

$.15 .001) 0+10$

$.23001 .30+10$

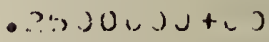

- $\{(1) 0(1) \div 0+(1,9$

- $3500100+0 ;$

$40(0(1) 0+0)$

$.4,00)(10+u)$

- s0000noto?

- ह 1) ง $) \div 100-111$

$.1 ? 00) 00+0 z$

$.1303000+13$

$.24 .70000+20$

- $\because)(,(\circ)) a+(1)$

- $3 t,)(1,1)+1)$

$.42001(12+0)$ 
$.43130000+()$ . $1405200+00$ - $1400000+(0)$ - $1 ? 622 \mathrm{CO}+60$ $.3100000+12$ $.1125900+02$ $.1000000+i 1$ -.207400!)-11 $.1000000-01$ $.3587000-01$ $.4000000-01$ . 7267000-01 - :000000-01 . 393000-01 - $1600000+00$ - $103 ? 100+30$ . 25\%0000+c0 - $1062300+00$ - $3500000+30$ $.1036700+00$ $.4930000+00$ - $78=2000-1) 1$ - $6400300+00$ - $124000-01$ . $3100000+00$ - $3361000-01$ . $1010000+01$

-.1154300+0u - $1000000-01$

-. $\$ 736000-01$ . $4000003-01$

- .1252000-01 - $9000000-01$ $.1770000-01$ - 1 . J COC O+00 - 375200$)-01$ - $2500002+0$. . 4029000-01

. seOCOŨt) -, $5.31000-.1$ $.4900000+00$ - i7 ig CCO-01 - i,400:00+00 - $; 635000-01$ .3100000+00 $.54(.700)-1)$. . $1000000+01$ $-.14 .1400+1)$ - $1000000-01$

- $1335300+011$ -4.) $000(1)-01$

- -34́tc000n-31 $\because 000000-11$ - .47 $7600,0-01$ - $150 \mathrm{CON}+00$ - . 20 8.9u0 0-01 . 2ग0(i)i - icyocon-0z
- $3 t .00000+00$

- $1000000+01$

- 3t,00000+00

- $10000(10+01$

- $360 \mathrm{cuj} 03+03$

- 1)00000+01

- $3630600+0 J$

- 103000.01

- 45c000u+co

- $1000000+01$

- 49c0000+00

- $1000002+01$

- $4300000+00$

- $1300000+01$

- 4ccJ000+00

- $1000000+01$

- 4 э COOUO too

- $1000000+31$

- 4 scocosor

- $1000000+01$

- 49cu000+00

- $1000000+01$

- $47601703+07$

- 1:00u0istul

- $4 \%(30 j)+0 j$

- 10000001

- $4500003+00$

- $10000 \ldots+01$

$.6403000+0.3$

- $1000000+01$

. $6400000+00$

- $10000010+01$

-6400000+0?

- 1 vovuoutol

- j4500útor

- 1u Julustol

$.54011001)+30$

- 1)0?300+01

- 64c00uutuo

$.1000(1)+01$

- $0460000+0$.

. 130000$)+1)$ ?

$.640000 u+0 u$

- 1$) 00000+01$

- E4corinotoc

- 100000$)+3$

- $6,+\mathrm{CCOO})+00$

- $1000(0)+01$

- E1c0:00+00

- 1 : $20: 200+31$

- 31000luton

$.1100030+01$

- $100000+0 i$

- $1 \mathrm{~J}) \mathrm{s}(\mathrm{0}+\mathrm{0}$ !

- $4100(0)+33$

- 1) 000(s)+01

- $12000 \mathrm{0}+0$

- 13$) \sqrt[3]{3} 3+3$
$.8600000+00$

$.6000000+00$

$.4800000+10$

$.3 \cos 1000+00$

$.0000000+10$

$.5400=0 \mathrm{c}+100$

$.1000000+01$

$.1000000+00$

$.2000000+00$

$.3000000+00$

$.4000000+00$

$.5000000+00$

$.500003+20$

$.70)(: 0)+00$

- 3co0i. 3is+00

$-\rightarrow \operatorname{con}(3 j 1)+0.2$

$.10 i) r(00+j 1$

- $10(1) 000+00$

- ?w(1) $30+00$

$.3000(00+00$

$0+3)(3), 1+0$

$.5000 \div 30+00$

- $600000+10$
$.6004(19)+3)$

$.7003000+00$

$.7300000+0)$

$.7000000+00$

$.7000000+00$

$.7300000+20$

$.7000000+0 !$

$.7900000+00$

$.700 .0000+(10$

$.7000000+00$

$.7000000+00$

$.80000 .30+00$

$.3000000+03$

$.8004(300+50$

- $3000000+01$

$.61 .30 \div 10+100$

$.7,10000-.31$

$.1400000+00$

$.2100000+00$

- $2830000+100$

$.350 n 000+0)$

$.4200000+\therefore$

$.4 \geqslant 00000+(0)$

$.563 .1000+3$

$.63(i)(6)+i 0$

$.7) 0(100(1+0)$

$.30,0000-.1$

$1 \pm 10000+00$

$.2 .4003000+(10$

$.32000(10+.00$

$.80,0000+0.2$

$.4) 50(00+1, ?$

- (3) $30000+00$

$.480 ; 003+i)$

- $310,0100+03$

$.56000(0+.1)$

-2)000(10+3)

$.04100 .3+1.9$

$.7201101)+(\div)$

$.8) \operatorname{man}(0+1)$

- (.) $) 0000-1$

$(1+(3))(1+11)$

- $27000100+0)$

. $36009040+i 1$

$.4530190+13$

$.9000013+0.1$

$.9000000-i 0$ 
- sE. $00000+00$

- $10 \$ 3000-01$

$.4900000+00$

$.1910(10)-31$

$.040 \mathrm{CO} 00+00$

- 240600$)-01$

- s1000ujoto

- et tiscco-O 1

- $1000000+01$

- . $262010000+00$

- $1000000-01$

$-.1960500+00$

$.4000000-01$

- $1442100+00$

$.9000000-01$

- $1031900+00$

$.1500000+00$

$-.7137000-01$

$.2500000+00$

-.472:000-J1

- $3500000+i 0$

-. $2917001-01$ . 440000u+Co

$-.1574300-01$ - $6400000+000$

- $5420000-02$

$.3100000+00$ - $2300000-03$ $.1000000+1$
$.8100000+00$

- 1 ) c0000+01

- $r 100000+c 0$

- 1$) 00000+01$

- $8100000+00$

- $1030(1)+01$

- 31c0000+oj

- $1000000+01$

- $8100000+00$

- 1 C CUOUO+C1

- $1300000+01$

- $1300 \div 00+01$

- 1$) 30000+01$

- $100000 J+01$

- $1000000+01$

- 19000coti1

- 1:000000+1)1

- $1000000+01$

$.1000000+3$

- $1060000+01$

- 1 U0:000+01

- $1: 300000+01$

- $10 \mathrm{cov} 0 \mathrm{0}+01$

- 1 : $100(\mathrm{~s})+J 1$

- $10 \cos (10+01$

- $10 \mathrm{cac} c 00+01$

- $10 \mathrm{CO} 000+31$

- 1 ग ง $000+01$

$.1000000+31$

\begin{tabular}{|c|c|c|}
\hline $.7000000+00$ & - $9000000+0$. & - 630$) \cdot(n)+r 0$ \\
\hline $.3000 .90+00$ & $.9 .2001)+3+00$ & $.7 ?) n(0), 0+12$ \\
\hline $.9(0003)+00$ & : $: 0001: 01+90$ & $.2130 .19+\div$ \\
\hline . $1000000+01$ & $.9000030+00$ & $.0103000+110$ \\
\hline $.1000000+00$ & $.1000000+01$ & $.100)(0.00+00$ \\
\hline $.2000100+00$ & $.10 .0000+01$ & $\cdot 2,0000101+10$ \\
\hline $.300000+00$ & $.1000000+01$ & (3) ) $3(n+)$ \\
\hline $.4(00) 00+00$ & $.1000000+.11$ & $.4 .100000+(9)$ \\
\hline .500000$)+00$ & $.1000000+3$ & ( ; ) $000(1+1) .3$ \\
\hline - $50001,00+01)$ & $.100 .00(3+) 1$ & $(\because), 0) 00,+10$ \\
\hline $.7003 .1019+00$ & $.10301 .3+1$ & 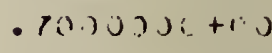 \\
\hline $.5 \mathrm{CJOBDO+00}$ & $.10000(1)+1$ & - $40300 n(1+1) ?$ \\
\hline $.8 \cos (1000+00$ & $.1000000+01$ &.$(;) 0002(1+10$ \\
\hline $.1000000+01$ & $.10000 \cup v+01$ & $.1000000+01$ \\
\hline
\end{tabular}


HIO : SRLINE FITTING MRELEEI

ऽ 1

- 3000000

- $) 000000$

$.1414:(2)+20$

. j000000

- $20,3000+20$

. 0000000

. $2449500+00$

- 0000000

- $2828400+00$

- 0000000

$.316: 2300+1) 0$

- $00 j 0600$

. $3454100+00$

$.1000000-04$

$.3741700+00$

- $6,00000-04$

- $4000000+00$

$.2 .200000-(.3$

- $4242600+20$

- $5100000-03$

- $: 472100+00$

- $1000000-0 ?$

- $4590400+010$

- $1730000-0 ?$

- यt8900u+co

- :740000-C2

- bu: $\rightarrow 0 n 0+00$

- 11$) 0000-02$

-:j20150J+10

- $5330000-0 ?$

-, $477200+130$

- $3000000-02$

- उús $9 \mathrm{C})+30$

- $1005002-11$

-j8.51000+00

- $13 \cdot 32)(1)-4) 1$

- 2000 unotí

-1753300-31

- $(1(44) 0+0$

-21:5)0 - J1

- 324000+vor

- $27002.30-i 1$

. (48)700+i)0

- 327/300-01

- $633200+j 0$

- 3930000-01

- v782300+J)

- $\operatorname{le}(6) 00-01$

- $65320200+0 u$

- $9,4 \cdot 37,000-01$

- $7711 \mathrm{CO}+0$

-6400000-01

$.7211103+13$

- 740 $\neq 000-01$

- $3+3500+00$
$-1000000+31$

- cuocous

. 1301000+01

- 0000000

- $1000000+J 1$

. 0000000

- $1000000+21$

- 00cucico

- $1300000+01$

- cocjes:os

- $1000000+31$

- Co0000:

- 1300600+01

- c000000

- $1000000+01$

- ouccouo

- $1000000+01$

- 6000000

- 1 liojoccotol

- co000( )

- $1003(00+01$

- $) 000000$

- 1$) \mathrm{Cu}(3(3)+3$

- 1 J) JuJ-u

- $1,00(50+01$

- 50isuco-0 it

- $103000 \mathrm{~J}+\mathrm{J}$

- $200000-03$

- $13: 2000+31$

- $5100000-02$

$.1000001+01$

- $100000 u-0 \%$

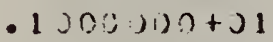

$.173)(0)-3 ?$

- $10 v(3) j u+v i$

- 274$)(30-3 \therefore$

- 1 s.juvudat J

- 41)?00J-1)2

- $1030000+01$

- 533. $3000-02$

- $1000000+01$

- $3 ; 0003)-03$

- $1.0000 u+01$

- 1 ) $500 j-01$

- $1300003+01$

- 1.382$)(0)-1$

- $1000(0)+01$

. 17580$), 01$

- 1 (1) $0000+01$

- $21(1500)-01$

- 1 i) couan+u 1

- $2700000-01$

- $1300005+01$

- 327 OL u-01

- 1 1)ucustui
.0000000

- $c 000000$

- $2000.00-01$

- 2000000

-4000003-01

.0000000

- $0000000-01$

- oconoon

- 30u0000-01

- conoujo

- $1000000+c .0$

- onncionu

- $1200(i) 0+00$

- 0000000

- 140000100

- cisejajon

- leglisjotoo

- jnjo.100

- $1800 \mathrm{ojo}+\mathrm{co}$

.000100$.

- $\therefore 000$ outoo

- ccosoos

- É?0) $10 n+00$

- ocunjol

- $240 \mathrm{a} 000+00$

- cuuviso

- 260 (:000+00

- 0000000

- $2300000+00$

- 0 cuovor

- 30003$) 0+00$

- cociovino

-3?Cᄂ)30+00

- 0.30 .100

- s4arisonatro

- cojocitjo

- 36,00(.)j+ou

- cosis.

- 38)0( $0+00$

- $00000 j 0$

- 40000 votoo

- 00000313

-4200(1)0+00

- $10000 j 0-04$

- 4+00000+00

- 501)0000-04

- 46\%1,000+00

- 2 2) vo $)(0-13$

. 48:JOC. $00+00$

- $5100320-03$

-51000(3) 30

- $1000000-02$

- $52(193010+10$

- $1730000-02$

$. ; 41)(1) 3+00$
.0300023

$.000(5)$

$.4000000-.)=$

- 0000000

- 1 ecocoro-os

- 00000ua

- 3602000-0?

.0000000

. $6400000-0 ?$

- onucoun

- $1000000-01$

$.030000)$

$.1440000-01$

- 0000000

- 1 cgrouu-i) 1

- 0000000

. 2560091)-.; 1

- ciovavo

- $32400(0)-(1$

- 0)0) 00

- 40 onou-i1

.0000000

-4.4413000-01

. 0000000

.576000101

.00000100

. $6760000-01$

.0000000

-7.340:100-01

. 0000000

- oncoson- 1

.00ulcon

- 10 年c00+c;

- Ojosico

$.1156000+0.1$

- jolocicos

- $12 \sec (100+60$

$.000000: 3$

$.1444000+0 \%$

- $000 j 00$

- Ie $01,000+0$ í

. 0000000

$.17(4)(0+i) 0$

. 00 jomoj

- $1936000+0)$

- 0000000

$.211(000+01$

- 0000000

$.2304000+00$

.0000003

- $2.50+030+0.5$

- 0000000

$.27040+0+01$

. 0000000

-?
- ) 300000

$.100030)-3+$

- () ) 20314

$.2 ? 00017-03$

$.5100000-03$

. $1000301-0 ?$

- $17^{-2} 3100-12$

$.274(0 ; ;)-3$

. $+1(1,1)(\cdot i)-1,=$

- $53 \therefore 30(0-0) ?$

- 4$) 000: 0-1: 5$

$.10 \cup 5 J(0)-31$

- $132 ? 000-11$

$.175800 .3-14$

.?145) 1-11

$.2700100-71$

- 2.277): 19-1

- $3 y 30 \cup 00-11$

$.40(.0,050)-01$

$.54 \geq 7030-11$

$.6 \cdot 400000-1) 1$

$.7403000-1) 1$

- s3 $13002-01$

$.97 .2410 .1-71$

$.11253) ?+(1)$

$-120,3) n+1 ; 1$

$.14: 361,1)+r 1$

$.1574(20)+i .7$ 
- 3513$)(30) 1$

$.783301+110$

- 1734)00-úl

. $7015303+i 0$

$.1105 \neq 0 \%+30$

$.7745030+10$

. $1250000+i 0$

$.737+100+i j 0$

$.1406100+00$

. $3000000+30$

$.1574600+00$

- $3124000+00$

- $1756200+00$

. $3246200+C 0$

- $1951100+00$

$.3366600+00$

- $2160000+00$

$.3485300+00$

-?383300+1 J

- $4602300+00$

- $2621400+i 0$

- $57178 \mathrm{CO}+\mathrm{UO}$

- 2:375000+00

- $38313(1)+(1)$

- $314+300+00$

- $3344300+00$

- $3430000+i 0$

- $7055430+1,0$

$.37325 \mathrm{co}+\mathrm{uu}$

- 716520100

. $4052200+00$

. $7273603+10$

-4383800+un

- $7380800+00$

. $4745500+00$

- $3486400+00$

- $5120000+00$

- $7591700+00$

. $551.3700+00$

-3695400+(10

. 5927001$)+i 0$

-37931)0 0 +cio

- 360 UOD + U0

- ง3 $93500+(10$

- $6.314700+00$

$.1000000+1) 1$

$.72 \div 6.300+100$
- $33.301:(5)-0:$

- $100(. j)+01$

- 46t $5(.)-3)$,

- $1203000+J 1$

- 5437(0)-01

. $1300 i 00+32$

- En+oju00-01

- 1 ju $0000+21$

.7409000-01

- $1000000+01$

- ग513000-01

- $10 \mathrm{c0000+01}$

- $5734 \mathrm{C}(0)-01$

- $1000007+01$

$.110 j 900+10$

- $1000000+1) 1$

- $1250000+00$

. $1000000+01$

$-1+301(0)+0$

- $1000 \cup 00+01$

- $15746: 3)+00$

- $1000000+01$

- $175 \%, 2 \pi 0+00$

- $1000600+02$

$.1: 31.0)+0 i)$

- $1000 \hat{0 u}+1$

$.216000)+0(1$

- 11) Uudo otol

- $238.3300+00$

- $10000.00+i 1$

- 2i $2140 u+00$

- $1100000+01$

- $2875000+00$

- $100000 u+01$

- 3144301$)+00$

- $100000 n+01$

- $3430000+00$

- $10300010+01$

- $3732500+00$

- 1$)(0001)+01$

- $405 i \geq 00+00$

- $100 \mathrm{uoriot} 01$

- $435318313+00$

- 1 J Ju0(00+1)1

$.4745600+00$

- looloviuto i

$\Leftrightarrow 120001)+00$
- 274 (a) 1$)-1) 2$

$.3600000+00$

.410)(01)- $5 ?$

- (5) हो (1)0 + +0

- ग83

- 000000 (j) J

. $8000(1, j u-02$

- $62000 \mathrm{vot} 00$

- $1065000-01$

-ij4000:20+00

- $1332000-01$

. $0630000+00$

- $1750000-01$

- 6 Buncon+o0

- $21 \div 50 \cup 1)-01$

$.7000000+00$

$.2700000-11$

- 7200ruvato

- 327?ก0n-0 1

- 7400000+00

- 345000 a-1)1

- 700uvootoo

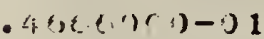

- 78 J $(1,0)+00$

- $54\{, 7(0) 0-31$

- escouvon+30

- $0400000-01$

- 32500uj+00

.74u9000-01

- $8400000+00$

. $3513600-01$

- 860.0000+00

- $9734000-01$

- 83800000+00

- 11 ก5suotoo

- y $\operatorname{coO} 000+00$

- $1250000+00$

- $9200000+00$

- $1400100+00$

. $9400000+00$

$.1574600+1) 0$

- 16u0ruotoo

- $1756200+00$

- $3000(0)+00$

- $19 \therefore 1180+00$

- $1 \mathrm{JuOC}(10+1) 1$

-? 1(.0000)+ jo
- 0 oń) )

$.3136 .00 .0+0$

$.17)(2 j)+\cdots)$

-) D01000

- 33(34)1, 1. 21)

- 00000001

- sencison+.sis

. 0 uvo(ji)

. $3844005+31$

. 0000000

-40(ít)

. 0000000

- $4356000+01$

- 0000000

- te $24000+(:)$

- 1) $000(00)$

- $7500000+6 i$

- .300000.)

$.5134000+00$

- $10000300-04$

- $5476.00 \Omega+00$

- ivivojo00-1)4

$.5776 .000+c 0$

- $2200000-0$ ?

- 608400u+00

$.51: 0000-02$

- $4400000+00$

- $1000(100-42$

- $6724000+00$

- $1730000-02$

$.7056000+100$

$.2740000-02$.

. 7396000+00

$.4100000-1)$.

$.7744000+0(1)$

- $5830000-0 ?$

. $3100000+00$

- 8000000-0?

$.8464000+00$

- 1 OE5000-01

- ह5.36000+00

- I 38а000-01

- $9216000+00$

$.1758000-01$

- 9504000+0u

- $2195000-01$

- $100 u c 00+01$

- 2. $70.06(1)-01$
$.171(0)+1)$

- 21030$)+(1)$

. $\Rightarrow+43(20+0)$

- $26 ? 1+00+00$

. 2 ค 750 i) $0+n$

- $31+4+30 y+00$

- 343000n+2n

- $3732010+1)$

-4052?(1) $(1+00$

$.4359 \mathrm{sic} 0+40$

$.47463500+100$

$.5120000+00$

$.5513700+00$

- $5927000+04$

$.53600(00+01)$

$.6914700+00$

$.7200000+00$

$.77 .35400+10.2$

$.83(15.30(0+0)$

- 8ร. $47400+110$

- cala $12905+0$

$.1000000+01$ 
HPI7: PELYACNI:

?. 3

6

- $10 J 0000+i) 1$ - 32 знग $70+1$

- ‘s $75400+60$

- 30(14 $33 \%+1$

-.351J6, $10+00$ $.24236 .70+01$

$-.9911100+00$

$.146,1>50+01$

-. $3092200+00$ . $7890100+00$

$-.7071105+C:)$

- $380500 u+00$

-.5477500+u0 - $1553500+00$

- 4535.10$)+i c$ . 4\%31000-01

-. $3990200+00$ - $\rightarrow 740000-0:$

$-.15043 \mathrm{cn}+30$ - 1 J0300-0.; - 00,0000

- j0c0irot

- $15 E+300+00$ -610C(D) - 3 - 3030?00+06; -.74)000-0? . $4535,900+i 0$ - 4c)31000-01 . $5877900+00$ - $1558500+00$ $.7071100+00$ - $3805000+00$ - $3030 ? 00+10$ - 7r9010j+ca - 3910100+u0 - $146,1750+31$ - 951053$)+i J$ .2443670+01 - पह $75 \mathrm{CO}+\mathrm{CO}$ - 345,4330+0 1 - $1000000+01$ -t.jes8.770+01 - v0 00000

-. $1550314+05$ -) 000000

- $1550.314+C 5$

AHPRCKI HATICH HRE BU U

- 1):10000 + 1

$-.4 \cdot, 6.31: 20+01$

- $100800 u+01$

$-. j 64+320+1) 1$

- $1, j 00000+01$

$-.31336+c+01$

$.130100 \%+.1$

$-.1607270+.1$

- 130000$)+01$

$-.7436 .700+00$

- 1$) 00(. j 0+01$

-. $2583500+00$

- $13 \mathrm{Cu} 03101$

$-.9733000-01$

- 1 i) $00003+01$

$-.2324 \cos -01$

- $10 \mathrm{cocos}+\mathrm{J}$

- $306.00 . j-0$ :

. 1$) \partial(., 0)+01$

- $1000000-0 j$

- 1 vo(i) + 1 - cosrous

- 1 juvuj+al - 1 त C $(:))-j \leqslant$ - 1 ग) Ju( $)+01$ - 3๘60(0)-0? - $100000+01$ - $2324 \mathrm{CC} 0-01$

- $10000010+01$ - 975,3000-01

- $10 \mathrm{CCONO}+01$ - ? $\rightarrow$ ล 50$)+00$

- 1 : icioisonto 1

- 743:3(10)+3is

- $1000000+01$

- $150 ? \therefore 70+01$

- $1000(10)+j 1$

- $1133640+01$

- $1030000+01$

- $5046 \geqslant 20+01$

- $1: 30000 J+01$

- 3563120+01

- 6000000

$.304 .4034+05$

- 0000000

- $3(44034+05$
-.1570 . $00+01$

$-.1413723+01$

$-.125 E(40+1) 1$

$-.104 \cdot 9 \cdot(0)+01$

$-.9+24600+00$

$-.73640180+00$

$-0.2 \times 3 \times 30+30$

$-.4712+90+00$

$-. \$ 141, .70+20$

$-.1570 .03+20$

$.0(4) \div .03$

$.1570 .100+10$

$.314180 i+00$

$.4712400+00$

$.0283200+00$

$.78(5400 j+00$

$.54 r c_{+} !(1)+00$

- $100.15020+01$

$.1250640+01$

$.1413720+01$

- $1577(1501)+01$

- 1600300+04

$.1000(0) 0+04$
$.240 .7+10+31$

. 1 ำ

$.157 \cdot 1+0+31$

$.120 \div .332+.1$

$.483 \pi 600+30$

- $013 x, 10+0,1$

$.3947520+0)$

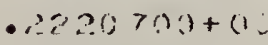

(1) 51910001

- 24 to (1;) $)-1$

$.000(21)$

$.2467040-01$

$.9370000-01$

- 른 $700+00$

$.36475(.0)+00$

- 61 : $15500+00$

- $x$ is $\left.2 \pi 0_{1}\right)+(0$

$\left..120 .70^{-2}\right)+(11$

$.157+1+1)+01$

$.199 x j+11$

$.2161400+01$

$-.3141533+04$

$.31415 \% 3+0$.
$-.3,75,7 \ldots, 2+1$

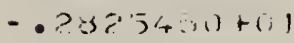

-.15834450+11

$-.1336 .30 .+9$

$-.4371700+0.9$

$-.4 \cdot 447(?)+i$,

$-.24 \div 0 \div .30+(\cdot)$

$-1(1025)+?)$

$-031(1)::-6$ !

- $\because \cdots:)(1)-$

$.190916)$

- 33300.100

$.310130-1$

$.1046540+1.0$

$.2450(0) 0+10$

$.4(2 / 447010+1)$

- $6 \because 717(\mathrm{atcij}$

- $13 ? \div 3+2+61$

- $1 ? 34+33+21$

$.242 .34 ! 3+11$

$.3 .37: 5730+91$

$.742230 .2+i 14$

- $74 \approx 2: \because+34$ 


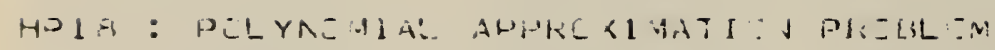

536 .

$-.1000000+011$ - 00 3 $3070+01$

$-.103030)+(0$ - $51>0) 580+21$

- (1)? $1100+(0$ - it $301+50+01$

- $98 \approx 2900+00$ - 365 cino+ 1

- . उ6 उ5800+10 - $3031070+01$

-. $5510 \varepsilon 00+00$ . 24 ') $3670+J 1$

-.7297800+00 . $2031110+01$

-.90483.30\%+J0

- $1630100+01$

-.9703100+.10

- 131)1710+i1

-.34? $3300+.36$

- $10 \% 1410+01$

- . 1390:0.1+:0

$.733010 \mathrm{Jt})$

$-.8705100+10$

$.5747300+i 0$

-. ?34 $3700+v 0$

- $+4 j 140+0 i$

- . $6345500+00$

$.32313(0)+00$

$\left.-.0 .37+2.00+0^{\prime}\right)$

-? 2का $100+00$

- .53 $57900+10$

- $155 \operatorname{sis}(0)+10$

-.35(30)u+1)0 - $1022500+j 0$

$-.+317 \div \mathrm{CO}+00$ - $3 ; 400)-01$

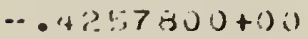
- $17420(0)-1) 1$

- $36 \cdot 12(0)+10$

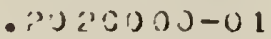

- . $30102000+0.2$ - $1740000-0$ ?

$-i^{3} 4 s \varepsilon y 00+(10)$ - :30, $3000-0$ ?

- $1073500+1) !$ $.126(00)-)=$

$-1253.300+21$

- 5 5000J-01

- $E ? 7+000-01$

-’) $10001-04$

-.10.02012

-i) U110600

- (:27.) $2(1)-) 1$

- $600000-1) 4$

$.125330 .1+00$
- 1110000001

- $5563120+01$

- $1000000+.11$

-. $7797500+01$ - $1000000+01$

$-.6302870+01$

- $1000000+01$

$-.504 .5760+01$

- 10 uvuvutol

$-.3999410+01$

- $1000000+01$

$-.3133640+01$

- $1000000+01$

-. $2424753+01$

- $1300000+01$

$-.1550330+01$

- $1000000+01$

$-.1330410+01$ - $1000000+0$ i

-.1 jа - $100(00)+1$

- $74363 v 0+00$

- 1 (ij) $030+01$

- .526 o $700+01$ - 1)iJ000+01

- .3135300+6u - $130000+21$

$-.2 .43670 j+00$ - $10000[3+01$

$-.1577100+00$ - $10,0000+01$ -.979.30no-01 - $1000000+01$

- ‘78?C!) - $1000000+01$

- 32) $+100-01$ - 1 (1)00000+01

- 1 4i, is -01 - 1 v000000 +01

$-.7 \% 1000 \mathrm{~J}-3: ?$

- 1.) 00000+ 1

$-.320: 200-0.3$

- 1.500001$)+01$

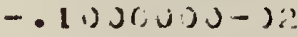

- $1000000+01$

- . $\geq 4 \operatorname{coc}(40-3 ?$

- $10 x(0) 0+01$

- 3.)0000)-0a

- 10$) 00(0+01$

- $\cos 00(3)$

- 1$)(0,0)+J$

- cosouson

- $10.00000+31$

. 000,1000

- 1 vocricoto1
$-.1573500+01$

$\ldots .15 .7900+01$

$-.1445130+01$

$-.1382300+01$

$-.1319470+01$

$-.1250040+01$

$-.119 .3010+01$

$-.11301790+01$

$-.100 .3140+01$

$-1(1) \div 10+01$

$-.94 .24(i) i)+00$

$-.3790500+00$

$-.816+1100+00$

$-.7530000+00$

$-.0911500+00$

- . $021-1201+00$

$-.505+700+00$

$-.5026 .50+00$

$\cdots+3+38.200+00$

- $; 7 t: 2,400+00$

:

$-.3141 .00+00$

$-2.5132010+00$

$-.1835000+00$

$-.1230600+00$

$-0.23,3000-01$

$.000(100)$

. $0 ? 3 j(10) 01$

$.126 x+0)+00$
$.2407+15)+31$

$-.3375,7,2)+01$

- $2 \% ? ? ?(3)(1)+11$

$-.34 \% 5) 5+01$

- $2083.3410+01$

$-.3018030+01$

$.1910760+01$

$-.2641 ? 40+01$

$.17+1000+01$

$-.22971 \div 0+11$

$.1575140+01$

$-.19834400+01$

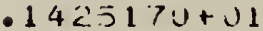

$-.17013 x, 1+1$

$.1275100+01$

$-.1440060+11$

$.1140+30+01$

$-.1 \leq 1 \div 30+1$

$.1010533+c 1$

$-.101(: 011)+1$

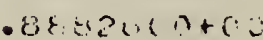

$-.3 .371700+00$

$.7737: 100+\ddot{0}$

$-.680656 u+.10$

$.66710(10+00$

$-.5444700+00$

$.5084900+(10)$

$-.428363(00+01)$

$.4776900+c 3$

$-.3301500+20$

- 39473010+60

$-. ? 4 \times 31) \div 31+(10$

$.3107800+0 ?$

$-018(-3)(2+3)$

- $2005000+00$

$-.1270 .1010+10$

- $19.34400+06$

$-40.8(5)-11$

$.1+21 ? 23+4$

$-\because(3), \cdots(i)()-(1$

$.5870000-01$

$-.3101 .10,1-: 1$

. $0317000-0.1$

$-.15 \div \therefore 10,-1)$

$.3553000=01$

$-.670)(100-11$.

$.1571000-01$

$-.1430: 160-3)$

$.3550000-0 ;$

$-.3500000-03$

- $0(100)(0)$

-1) 1rni:i

. 305500010 0"

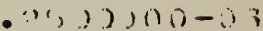

$.157+00.9-11$ 
- 2501000$)-113$

- $13733 \mathrm{Cn}+0$

- 126030$)-(1 ?$

- $2436 ; 00+00$

- 39960.3, J2

- $3 \mathrm{~J} 9200+: 11$

- 17400तก-0द.

- 3ij $1: 0 u+j u$

- ?) $2, J 0:-01$

- 4?57300+0u

- $3742 \mathrm{CO}-01$

- $43175 \mathrm{CD}+\mathrm{U} \mathrm{J}$

- $6384000-C 1$

- $5353.300+i 0$

- $10: 2600+00$

- $5877200+0 n$

- $1555500+00$

- $.374 .00+.17$

-?:3130J+u

.63455(0)+100

- 3231 s.jotuo

$.1289700+00$

.44 j $140 u+00$

.? ग 10$)+00$

.

- $3940200+00$

- $7390139+03$

- $24+3300+v u$

- $10 ? 1410+01$

. $376.3100+00$

- $1301710+01$

- $9043300+00$

- $1635102+01$

- $32.37800+00$

- $2031110+31$

- $\rightarrow 510$ < $100+1) 0$

- $2+9357 \mathrm{C}+01$

- $\cos 580(0+i)$

- $30 \Xi 1(7)+1$

- $\rightarrow 3 c^{2} 2400+00$

. $3550 i 30+31$

- अ921100+.30

. $4361450+01$

- भग 70.31$)+00$

$.5170883+1$

- 1 วगUn日+ 1

- $003070+01$

- J.j00i:

- $15,50314+J 5$

- vojoún

- $1550314+45$
- 312:0(3) 01

- I Jus $(r ;)+01$

- $24 c 0(1,2)-0$;

- $100 u\left(c^{2}\right)+0$ ?

- 1200001$)-0$ a

$.1) 00600+1) 1$

- 3 jes.5-1)?

- 1 10000u+01

- 7:1200.)-02

- $1000000+01$

- 1 ن46000-0 1

- 1 j coúutol

- 320;000-01

- 1 0uis?

- 57820v0-01

- $1000 \div 0+01$

$.273000-1$

- 1 Ju $200+01$

- $1577100+00$

- $100 r$ ouo +o 1

- $2435700+00$

- 1 ).3000 + 1

- is 35ju+0u

- 10 2300u+J1

- $52(6100+00$

- 1 1)301)00+31

- 7430 300+0u

- $1000(10)+01$

- 1 ) $2 \leqslant 30+01$

- 1 J 00000 +01

- $1390410+01$

- 10$) 0$ Uud + 01

- 1 ×50 350+01

- $10 v u(100+u 1$

- ?4247jis+01

- $1: 3 x 003+01$

$.3133(40+01$

- 1 voucuotua

- 3s<. $3410+01$

- $10 j 0000+01$

- $504076,0+01$

- 1 ) cu20u+01

- $5-1) \geq 370+01$

- 1 u0ucióto 1

. 7777500+01

- $1300(0)+01$

- $c 563120+01$

- 1) 300000

- 3.) $440.44+05$

- 0uisonoro

- 30441$) 31+05$ $\cdot 13(3,1)(3+10$

$.3 \div 2,310-31$

- 73$)$ U

$.251 \% 300+00$

-6:1979) $1-111$

$154040(1)-01$

$.31410190+0$

- $937: 1: 4)-1$

- $3101.0 .0-01$

- $3700 \cos 00+00$

$1+21: 1: 2+1$

- ว5:3311 $3-61$

$.+39 n \geq 10+00$

- $19.44 .14+33$

- 85 ; $4000-1$

$.002650+00$

- $2526,000+03$

- $565+200+70$

$.31 .77(6(1)(1+)$

-is? icisotuc

$.36+(36)+i)$

$0 \rightarrow 115,10+00$

-47?:3: :n+0)

- $7 \vdots 3)+40+00$

- $5634 \mathrm{Ad} \mathrm{Ir} \mathrm{I"}$

- 16 , s)

$.671 \cdots+1$

$.773 !(3 i J+31)$

- is $830(30)+00$

.101 UEsu+o1

$.100:-110+01$

$.106: 3140+01$

$.1140330+01$

$11: 0 \div 7 j+01$

$.127+160+01$

$.115,3310+01$

- 1250 4uto1

- $131 \div 470+01$

$.13 \times 2300+01$

$.1445130+1$

$.1507 y 60+01$

. $2273 \cdot 7(\cdot)+01$

$.1570(00)+01$

- $130500+34$

$.1000(1)+04$
$.2467+2)+31$

$.1435 i 7 J+3 i$

- $137: 1401+1$

$.174100)+(: 1$

$.1516760+5$

- $2(154+1)+111$

- 301 $130 . \because+11$

$.74 \because(15)+1) 1$

$.3+757 ? .0+1$

$.740 ? 20.3+.94$

. $740 ? \cdot 3 r . ?+2 t$ 


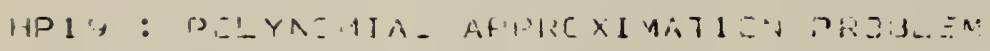

$103 \quad \epsilon$

$-.10) 01001+31$ - $5038070+11$

$-.2393123+30$ - 5e $15+40+i, 1$

- $19+20500+j 0$ $.51 ?$ (ด)

- $4955600+00$ . $4753250+) 1$

- .5721100+00 . $4361+50+1) 1$

-. cr $76000+00$ - $3994281+01$

- . $8202500+00$ - $3550430+31$

-.9759200+1)0

- $: 33.3220+1$

- . $09800+00$

- $31031070+1) 1$

- .360 2.;00+i)0

- 27? $2550+1) 1$

-.1510600+i.

- $24=3 \div 70+11$

-.34(veis)(1)

-225 3i,00+01

-. $.277300+0)$

- 2) $31110+01$

-.1177000+00

- $1325600+01$

-.0048;300+00

- $1 \in 3.0100+3$ !

- $310100+00$

$.1461750+1) 1$

$-.3763100+00$

. $1301710+01$

- . 850.742$)+16$

$.1155190+.31$

- $-2443303+j 0$ - $1021410+01$

- . $12705300+00$

- 35is5(300+00

- $\because j 00<05+i j$

- $r$ a(j) 1 (j)

$-.77018,00+00$ - $\cos 3 \cdot 3600+0$ i)

$-.7705100+00$ $.50837 .500+30$

$-.7501100+10$ .517070 J+11

- I\&C $7700+100$

- 145140)+10

$-.707110)+13$

- :3ujociotis

- .

- $\therefore 231+c 0+1) u$

- ter $131(01)+1)=$
$.1000000+01$

- $9503120+01$

- 1 ) ग Ji $30+01$

- $304410 u+01$

- $10.00000+01$

$-.779750)+01$

- 1)0(, $600+01$

- 7) $13410+01$

- $1000 v \omega D+01$

- $0302870+01$

- $1000000+01$

$-.5640320+01$

- 10 CE CuUto 1

-. 5) $407(30+01$

- $1000000+01$

$-.44547 j)+01$

- $1000\left(0_{1}\right)+01$

$-.3 \cdot 104413+01$

- 1) jucoutol

- . $23+3+30+01$

- $1300000+01$

- 3133r40+01

- $\left.10000_{2}\right)+.01$

-. ?.7( $1040+01$

- $10.03000+01$

-. $2424750+01$

- $10300.00+01$

$-.2122050+01$

- $1301000+01$

$-.1253330101$ - $1000000+01$

$-.1607270+31$ - $130300+01$

- $1390410+01$ $.13000(i)+01$

$-.1197620+01$ - $110000 \mathrm{jol}$

- $1023330+01$ - 1 ijo) $000+01$

-. $\quad 57^{7}(t) 11(0)+00$ - $10(10000+01$

$-.743 f_{3}$. $310+00$

- 1.j J00 0 o + 01

$-0.270420+00$

- $1000003+01$

- $3 \geq \ln 7$ (?) +0!

- $1000(j u j+j 1$

- $4391(i)+01$

- $1: 0100 u+01$

$-.36 .35 \% 00+00$

- 17.$) \div 0(2)+11$

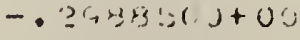

- 11) Ji( $(3)+1) 1$

$-\ldots+3,71) 0+00$

- $1: 2 ;: 11+3$
$-.15203+31+01$

-. 1507yÉ

$-.1476550+01$

$-.14451: 30+01$

$-.1413720+01$

$-.1342300+01$

$-.1350860+01$

$-.1316 .470+31$

$-.12 r^{2} 305 u+61$

$-123 u 40+01$

$-1235.20+J 1$

$-.1143+310+01$

$-.11(12,90)+01$

$-\therefore 1130 \cdot 80+71$

$-10 y+30,0+01$

$-.1003140+31$

$-.10 .16120+01$

$-.1005 .110+01$

$-.1733 i \cdot 00+00$

$-.34 \div 42: 00+00$

$-2=11(1000+00$

$-.3776 .500+00$

$-.043 .300+00$

$-.81105100+00$

$-.7+, 4: 0 n+0)$

. $72.1 .13+00$

$-.7 \because \cdots, 700+00$
$.2467+00+.31$

$-.3755740+31$

.? $36 \div 6: 3+31$

- .36.47:.. $.9+11$

$.2273000+31$

$-.342911 \cdot v+C 1$

$.218302: 30+01$

$-.7215100+21$

- $2033410+01$

-.3.) 13030+01$

$.19735 \because 0+61$

$-.2 \leq 5 \div 5: 450+01$

$.1 \geqslant 107.0+01$

$-.2+41.41+21$

$.13242+3+01$

-.245:5\%?+\%1

$.17416 .00+11$

-.ว?:71:1+1!1

- $165 \div \leq 39+01$

$-.21 \cdots+2,1+21$

$.1 ; 7 ! 1+1+? 1$

$-.13944) 1+11$

- $150 ! 17)+01$

-. $13.3930+1$

$.142317 i+121$

$-.17(1393)+31$

$.135 ! 150+0 !$

$-.157 \operatorname{Jrin}^{2} \cdot 11$

$.127: 1010+31$

$-.14468 .1)+01$

$.12 .90(0+)$.

$-.13003) 1+11$

$.1(4,1), j)+r ; 1$

$-1 \geq 133^{2}+7+1$

$.1074 .5100+31$

$-.1114 \therefore 71+71$

$.1010(-51)+11$

$-.1016) 10+31$

- $548+700+0$.

- . $2371 \ldots+100$

- $38.32 t: 00+(4)$

$-.93717 i 18+10$

- $320(1030)+01$

-. $75(2103+2)$

$.7737() 0+$.

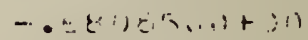

$.71(; 40.20+0 i)$

$0 .(.1) 31 \div .9+.00$

-56 $719(1)+19$

-. 5.54 $4700+00$

. $31<a .2)+9 i$

$-.43 .14720+121$

-, $1+5 \cdot i+3 i+\ldots$,

... $4: 33,0.3 \cdot 3+1+1$

- נ口? ? >n+i: 
. $2725900+00$

$-.6374200+00$

. .2819c0+00

- .6129100+00

- $1874400+00$

$-.5877900+00$ - $1558500+00$

-.5620800+00 - $1269400+00$

$-.5353300+00$ - $1022600+00$

- $5090400+i 00$ - $\$ 136000-01$

-.4917500+00 $.6384000-01$

-. $4534900+00$ .4931000-01

- .42578CD+00 $.3742000-01$

-.3971500+00 $.2782000-01$

-. J681200+00 .2020000-01

$-.3397400+00$ - $1426000-01$ -. $3090200+00$ $.9740000-02$

- . $7339900+00$ . $639 \mathrm{C000-02}$

-.2496900+00 .399 c000-02

-. $2181400+00$ - $2340000-02$

-. $1873800+00$ $.1260000-02$

$-.156 .4300+00$ . $6100000-03$

$-.1253300+00$ $.2500000-03$

-.9411000-01 . $8000000-04$

-.0279000-01 . 2000000-04

-.3141000-01 .0000000 .0000000 .11000000 $.3141000-01$ . 10000000 $.627 .7000-01$ . 2000000-04 $.0411000-01$ . $8000000-04$ $.1253300+00$ . 2500uno-v3 $.1564300+00$ $.6100000-03$ $.1873800+00$
$-.1969600+00$ - $1000000+01$

$-.1577100+00$ - $1000000+01$

$-.1249801)+00$ . $1000000+01$

-.9793000-01 - $1000000+01$

-.7577000-01 - $1000000+01$

-.5782 C. 0-01 $.1000000+01$

-.4345000-01 . $10 \mathrm{cc000+01}$ -.3209000-01 - $1000000+01$ -.2324000-01 - $1030000+01$

- .1645000-01 - $1000000+01$

$-.1130000-01$ - $100000 u+01$

-.7610000-02 - $1100000+01$

-. 4030000-02 - $1000,000+01$

-.306 $2000-02$ $.100000 u+01$

- $1810000-02$ - $1000000+01$

$-.1000000-02$ - $1000000+01$

-.51 cocon-0.3 - $1000000+01$

$-.2400000-03$ $.1000000+01$

$-.1000000-03$ - $100000 \mathrm{i}+01$

-.30c0000-04 - $10100000+01$

-.1000000-04 - $1000000+01$ - 0000000

$.1000000+01$

- c0000uo

$.1000000+01$

.0000000

- $1000000+01$

.0000000

- $1000000+01$

- C CCO000

$.1000000+101$

- $1000000-04$

$.1000000+01$

$.30 \operatorname{cov} 0 J-04$

$.1000000+01$

$.1000000-03$ $.1000000+01$
$-.6911500+00$

$.4776900+00$

$-.3301 .3130+10 u$

$-.6597300+00$

$.435 .2500+00$

$-.0283200+00$

. $3 \subseteq 47800+30$

$-.5965000+00$

$.356 ? 500+00$

$-.5654: 00+00$

$-.5340: 00+00$

$-.5026500+00$

$-.4712+00+00$

$-.436 .830+00$

$-.40+4100+00$

$-.370,0900+00$

$-.3455300+00$

$-. \therefore 141600+00$

$-.2827400+00$

$-.2513300+00$

$-.21 \subseteq 9100+00$

$-.1885000+00$

$-.1570300+00$

$-.1256,(0)+00$

$. .9425000-01$

-.6283000-01

$-.3142000-01$

$.0000000^{\circ}$

$.3142000-01$

$.6283000-01$

$.9425000-01$

$.1256000+00$

$.1570300+00$

$.1885000+00$
$.3197800+60$

- ?85? $300+00$

$.252(600+0.01$

- 2.22070n+00

- $1934400+00$

$.1668000+00$

$.1421200+00$

$.1104200+010$

$.92370000-01$

$.7994000-01$

-E $317000-01$

$.4336000-01$

.355300001

$.2467000-01$

$.1570000-01$

$.8380000-0 ?$

$.3951000-0$ ?

- $900000-03$

.0000000

. sa or 000-0."

- 3n5.3000-02

- kres $80000-0 ?$

.157 000-11

$.2467000-01$

$.3553000-01$
$-0.371500+00$

$-.2400500+00$

$-.212 .670)+03$

$-.1808300+.00$

$-.15 .23300+($.

$-.1270(8) .7+($.

$-.10465(10+3)$

$-356.607-91$

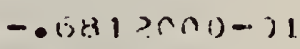

$-.53530 n n-11$

$-.41 .730)-01$

$-.3101000-01$

$-.2200000-01$

$-.156 .3000-01$

$-.10 t 4000-01$

$-.0700000-n^{\circ}$

$-.3860000-0 ?$

$-.1 \cdot 30000-0 ?$

$-.84000011-(: 3$

$-.250 .0000-1.3$

$-.3000000-04$

.0000003

$.30,0000-134$

- ?500001-i) 3

$.3400200-93$

$.10 \div 30,00-3$

- 3ystona-2?

$.67 .0000-0 ?$ 
$.1260000-02$ - $2161400+00$ . $2340000-02$ $.2436900+J 0$ - 359 cu00-02 . $274590 \mathrm{~J}+00$ .6390000-02 . $3090200+00$

. $7740000-02$

. $3387400+00$

$.1426000-01$

$.3681200+00$ . 2020000-01 -3i) $71500+00$ . $2782000-01$ $.4257800+00$ . $3742000-01$ $.4539900+00$ $.4931000-01$ $.4817500+00$ -6384000-01 . $5090400+00$ . $9136000-01$ . $5358300+00$ - $1022600+00$ - $50<0800+00$ - $1269400+00$ $.5377900+n 0$

- $15585 \mathrm{CO}+\mathrm{J} 0$ . $129100+00$ - $1894400+00$ . $6374200+00$ $.2281900+00$ . $6513100+00$ $.2725700+00$ . $6345: 00+00$ . $3231800+00$ $.70 ? 1100+c 0$ $.3305000+c 0$ . $\left.7283^{\prime}\right) 700+00$ $.4451400+00$ $.7501100+C 0$ $.5175700+00$ $.7705100+180$ $.5987300+00$ $.7901600+00$ - $6983<000+00$ - $5050200+00$ $.7390100+00$ $.3270800+00$ - 39055700+00 . $3443300+00$ - $102^{\prime} 1410+01$ . $26(1) 7403+00$ . $115.5190+31$ - $56,3100+00$ $.1301710+11$ $.3910100+00$
- 24 coc.00-03 - $1000 c 00+01$ $.51 \mathrm{CCO00-0.3}$ - $1000000+01$

- $1000000-02$

. $1000000+01$

- $1810000-02$

- $1000000+01$

-3060000-02

- $1000000+01$

- 4530000-0a

- $1000000+01$

- 7e $1000.0-0 a$.

- $10 \operatorname{coccot} 01$

- $1136,000-01$

$.1000000+01$

$.1646000-01$

. $1000000+01$

$.2324000-01$

- $1000000+v 1$

- $3209000-01$

- $1000000+01$

$.4345000-01$

$.1000000+01$

$.5792000-01$

- $1000000+01$

. $757701 j u-01$

- $1000000+01$

. $9793000-01$

$.1000000+01$

- $12498300+00$

- $1000000+1$

- $1577100+00$

$.10000(0+31$

- $196^{\circ}$ conotoo

$.1000000+01$

$.2436700+01$

- $1000000+01$

- $2988500+0 u$

$.1000000+01$

. 3030 $300+00$

$.1000000+01$

$.4391000+00$

- $1000000+01$

- $52607(1)+00$

$.1000000+J 1$

- $627680(3+0)$

- 1000uvotol

. $7436300+00$

$.1000000+01$

- $37611(00+0)$

- $1000(00+01$

. $1025330+01$

- 10 uUocotul

- $1197520+01$

- $1000000+01$

- $1300410+01$

- $1300000+01$
$.2199100+00$

$.43360(1)-11$

$.10(14)(1)-01$

$.2513300+00$

$.5317000-.11$

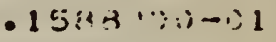

$.2827400+00$

- $79094000-01$

$.3141600+00$

$.9870000-01$

$.34558000+00$

-1194200+00

$.37 x, \cdot 900+00$

$.1421200+01$

$.4084100+00$

$.1663100+00$

$.439820 u+00$

$.1934400+00$

$.4712 .400+00$

$.22207100+01$

$.5026500+00$

$.5340700+00$

- $5654900+00$

$.5960000+00$

$.0283: 00+00$

$.0597300+00$

$.6911500+00$

$.7225700+02$

$.153 .300+00$

$.7854000+00$

$.81 \cos 100+00$

. $8482300+00$

$.3740500+00$

$.3110600+00$

$.9424500+00$

$.573 \div 03: 1+00$

$.1005310+1) 1$

$.10 .3 t \cdot 7.40+01$

- $1068140+01$

$.1099500+01$
$.25266: 30+0)$

$.2352300+00$

$.3157800+10$

$. \$ 503700+00$

$.3547800+00$

$.435 z \dot{2}(0+0)$

$.4775000+00$

-52 $2<1000+00$

-5634000+8j

$.61585: 10+011$

$.6871000+00$

$.7154900+0.3$

$.77 .37: 00+11$.

$.8300300+00$

- $3(820,00+61)$

$.94 .34700+0 j$

.1010 (1)

$.1074800+01$

$.11409 .30+n 1$

$.1200050+01$ $.22000(i)-.11$

$.31010 r)-11$

$.4127100-31$

$.5359(100)-11$

$.5412000-1$

- $850306 ?-61$

$.10455000+1.0$

$.12700(0+i)$

$.152: 300+1.9$

- $1803300+1,0$

-2.12: $70(0+0)$

$.24+0500+00$

$.2971530+(.0)$

- $901800+01$

$.3770501)+00$

$.42+n .000+0 J$

$.48447 x+10$

. 54 4 $4071: 1+\because !$

- $1.1: 3000+20$

$.6700 ; 320+10$

-?5. $62100+10$

- $\because 41-\therefore+(i)$

$.02 .371(1+.0$

$.101 \ldots 119+(1$

$.111+\div 79+01$

$.1 \geq 1+\cdot 7: 1+11$

$.13 ? 13,1+111$ 
- $14 \epsilon .1750+01$ $.9048300+00$ - $1636100+01$ $.9177500+00$ - $1825600+01$ $.9297800+00$ - $2031110+01$ - $9408800+00$ . $2253500+01$ . $9510600+00$ - $2493670+01$ - $9602500+00$ - $2752550+01$ - $9685800+00$ . $3031070+01$ $.9759200+00$ - $3330220+01$ - $9822900+00$ - $365 \cos 0+01$ - $9976900+00$ - $3994380+01$ - $9+21100+00$ . $4361450+01$ $.9955600+00$ - $47532.5 \mathrm{~J}+01$ - $9980300+00$ . $5170880+01$ . $9995100+00$ . $5615440+01$ - $1000000+01$ - $0088070+01$ . 0000000

-.1550314+05 . 0000000 $.1550314+05$
$.1607270+01$

- $1000000+01$

- $1850300+01$

- $1000000+01$

-? $122060+01$

- $1000000+01$

$.2424750+01$.

- $1000000+01$

$.2761040+01$

. $1000000+01$

$.3153640+01$

. $1000000+01$

- $3545430+01$

- $1000000+01$

. 3999410+01

. $1000000+01$

$.4458750+01$

. $1000000+01$

$.5045760+01$

. $1000000+01$

. $5646 \div 20+01$

- 1 J $00000+01$

$.6302870+01$

- $1000000+01$

. $7013410+01$

$.1000000+01$

. $77975 \mathrm{CO}+01$

. $1000000+01$

- $8644300+01$

- $1000000+01$

- $7563120+01$

.0006000

. $30440.34+05$

- (i) 00000

$.30440 .34+05$
$.1130970+01$

$.1279100+01$

$.1445637+01$

$.1162390+01$

$.1351150+01$

$.15705 t 0+01$

$.1193810+01$

$.1425170+01$

$.1701380+01$

$.1225220+01$

$.1501170+01$

. $1839260+01$

$.1256640+01$

$.1579140+01$

$.1084400+01$

$.1288050+01$

$.1659080+01$

$.131 \div 470+01$

$.1741000+01$

. $1.350830+01$

- $16243 \sin +01$

- $1382300+01$

$.1910760+01$

$.1413720+01$

- $1998590+01$

$.1445 i 130+01$

$.208 .8410+01$

$.1476 .550+01$

$.2130200+01$

$.1507900+01$

$.2273960+01$

$.1539380+01$

$.2369690+01$

$.1570800+01$

$.2467490+01$

$.1000000+04$

$-.3141593+04$

$.1000000+04$

$.31415 y 3+04$
$.2136980+11$

$.2207151+31$

$.24 r .5320+i 2$

-? $? 64124 n+C 1$

$.28525450+101$

$.30130 .30+.11$

$.3219160+11$

$.3429050+J 1$

$.3547860+01$

$.397574) 1$

$.7402203+04$

$.7402203+04$ 
HP2O: PCI.YNC YIAL APPREXIMATIIVV PHCBLEAA

2036

$-.1000000+01$ $.6088070+01$

-. $3998300+00$ $.5348170+01$

-.7995100+00 $.5615440+01$

-. $9983900+00$ $.5389720+01$

-. $5980300+00$ $.5170880+01$

-. $3969200+00$ - $4958770+02$

-.9955600+00 . $4753250+01$

-.9939600+00 . $4554190+01$

-.3921100+00 . $4361450+01$

-.3900200+00 . $4174990+01$

-.9876900+00 - $3944380+01$

- . $7351100+00$ - $3819790+01$

-.9822900+00 - $365 \mathrm{c}: 90+01$

-.9792200+00 - $3487840+01$

-.975)20u+00 . $3330220+01$

-.9723700+00 - $3178010+01$

-. .6954800+00 - 30 अ $1070+01$

- $7045000+00$ - zE 3 $3200+01$

- . $360 ? 000+00$ - $2752550+01$

- (1)5579c0+00 - $2 \varepsilon: 20710+01$

- i511) ग00 +00 - $2493670+01$

- $9400900+00$ - $2.371310+01$

-.9408800+00 - $2.25 .3500+01$

-. 335400+00 - $2140140+01$

-. $0207800+00$ - 2ns1110+u1

- . $3238300+0$ ! - $1926300+01$

$-.7177500+00$ - $1435600+01$ $-\rightarrow 114000+00$
- $1000000+01$

-. $5563120+01$

- 1 i) $00000+01$

$-.9094430+01$

- $1000003+01$

$-.8644300+01$

- $1000000+01$

$-.8212170+01$ - $1000000+01$

$-.7797500+01$ - $10000 \mathrm{CO}+01$

-. $7399760+01$ - $1000000+01$

$-.7018410+01$ - $1000000+01$

-.065:450+01

- $1000000+01$

$-.6302870+01$

- $1000000+01$

-. $5967690+01$

- $1000000+01$

$-.5646520+01$

- $1000000+01$

-.534)100+01

- $1000000+01$

$-.5046760+01$

- $1000000+01$

$-.4766460+01$ . $1000000+01$

$-.4453750+01$

- $1000000+01$

-.424.3200+01

- $1000000+01$

-.3959410+01

- $1000000+01$

-.37E095U+U1

- $1000000+01$

$-.3545430+01$

- $1000 \mathrm{CJJ+01}$

-. $7334450+01$

- $13000.70+.01$

$-.3133640+01$

- 100000.$)+31$

-. $94262 J+01$ - $1000000+31$

$-.2701040+31$ - $100000 \mathrm{Co1}$

-.?583530+01 - $100(j 0) 0+01$

-.2.424750+01 - $1000007+01$

-.2269370+01

- $1000000+01$

$-.2122000+01$ - $1000000+01$
$-.1555090+01$

$-.1539380+01$

$-.1523670+01$

$-.1507960+01$

$-.1492 ? 60+11$

$-.1476550+01$

$-.14608340+01$

$-.144513(+)$.

$-.14294 c^{\prime} 0+01$

$-.14137 ? 0+0.1$

$-.1398010+01$

$-.1382300+01$

$-.136 C .5 \subseteq 0+01$

$-.1350 \varepsilon 80+01$

$.1335180+01$

$-.1319470+01$

$-.1303700+01$

$-.1288050+.11$

$-.1272 .350+01$

$-.1250(3.40)+01$

$-.1240930+01$

$-.1225220+01$

$-.1209510+11$

$-.115 .3310+01$

$-.1178100+01$

$-.1162,390+01$

$-.11466+80+01$
$.246 .7400+01$ .. $3975700+01$

$.2418300+01$

$-.3780,+0+51$

$.2 .360 .690+01$

$-.3647800+01$

$.23215310+01$

$-.35=7320+01$

$.2273900+01$

$-.3429150+01$

$.2224,03)+11$

$-.3323200+.11$

- $2130 ? 00+01$

$-.3 \% 1=160+21$

$.2134000+01$

$-.3117516+01$

$.2083410+01$

$-.30180 .31+01$

$.204325 j)+01$

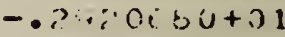

- $1998550+01$

$-.2825450+01$

- $19544.70+01$

$-.2722310+01$

$.1510760+01$

$-.2 t 41.940+01$

- $1867580+01$

$-.2552220+11$

$.182 .4390+01$

$-.2456200+01$

$.1782700+01$

$-.23803 .50+01$

$.1741000+1) 1:-2: 2471(10+01$

$.1540793+01$

$-02312010+01$

$.1659 \sin 01$

$-.512(0,0) 11+01$

.161 (3) + 01

$-.2 \sin 75)+(11$

. $157 ? 140+91$

$-013=4(40 i)+01$

$.1339910+01$

$-.1: ; 1: 2:+01$

- $1501170+01$

$-13.99(1+1) 1$

$.146 .920+61$

$-.17 f .5420+01$

- $1420171+01$

$-.1761 .91+01$

$.1357910+c 1$

$-.12 .6101+01$

$.1351150+01$

$-.1570560+01$

$.1 \geq 148 \% 0+01$

$-.1507750+01$ 
. 172 เ900+11

- $9043300+00$

- $1636: 1 \mathrm{CO}+01$

- $3983300+00$

. $1547090+01$

-.3910100+00

$.1461750+01$

-.\$837700+00

. $1379990+01$

$-.8763100+00$

$.1301710+01$

-.8686300+00

- $1226310+01$

$-.8607400+00$

- $1155190+01$

- .85264 Co+00 - $1086760+01$

-. $3443300+00$ . $1021410+01$

- . $358100+00$ - $9590500+00$

-.32708n $3+00$ - -3() $75900+00$

-.8181500+0 J $.3429400+00$

-.3090200+00 $.7890100+00$

$-.7936900+00$ $.7377100+00$

$-.7901600+00$ - $639960+00$

-.7804300+00 . $6426600+00$

$-.7705100+00$ .59873 Co+0

-.7604100+00 $.55710 \mathrm{CO}+00$

-.7501100+00 - 5176700+00

-.7346300+00 - $43038(2+0)$

-.7389700+u $.1+51400+00$

$-.7131300+00$ $4113700+00$

-. $7071100+00$ - is $150 \mathrm{CD}+00$

-.6557100+00 $.3503700+37$

-.6345500+00 - $3231800+00$

$-.6730100+00$ . $2370300+00$

- 化 $131 \mathrm{C})+i 0$ - $2725900+0 u$

-.6454500+ciu - $3445560+00$ - $\therefore: 374200+00$
- 1 cEeson+01

- 1)0 jul 01

- 1 $1533.31+01$

- $1000000+01$

$-.1725410+31$

- $1000000+01$

$-.1607273+01$ $.1000000+01$

$-.1455700+01$ - $1000000+01$

-.1390410+01 - $10010000+01$

$-.1291140+01$ - $1000000+01$

$-.1197620+01$ - $1000000+01$

- $1109600+01$ - $1000000+01$

$-.1020330+01$ - $1000000+01$

$-.9450300+00$ - $1000000+01$

- $87.61100+00$ - $1030000+01$

$-.8077000+00$ - $1000000+01$

-.74353(i)+0u - $1000003+01$

- $6830500+00$ - $1000000+01$

-.627E8cotoj - $100000 J+01$

$-.575410 J+00$ - $1000000+01$

-.5?66700+on - $1000000+01$

$-.4513000+00$ - $1000000+01$

$-.4371000+00$ - $1000000+01$

- $35 \mathrm{cs} 30+00$ - $100000 j+01$

- 3033500+00 - $1000000+01$

- $329450 J+00$ - $1000000+01$

- $238500+00$

- $1000000+01$

$-.2701360+00$

- 11$) 00000+01$

- .2430 $700+00$

- $1000000+01$

$-.2195300+00$ - $1000000+01$

- $1969600+00$ - $1000000+01$

$-.17647 \mathrm{co}+\mathrm{CO}$ - $1300000+01$
$-.1130470+01$

- 127:1 11+4)

$-.144 .3 .80+1$

$-.1115270+11$

$.10430+; 1$

$-.1 \div . .716)+61$

$-.104 .7560+01$

$.12090 .10+01$

$-.1374293+01$

$-.1083350+01$

$.1174730+01$

$-.1273230+01$

$-.1068140+01$

$.1140930+01$

$-.1218670+01$

$-.1052430+01$

$.1107620+01$

$-.1105090+01$

$-.1036730^{\circ}+01$

$.1074300+01$

$-.1114270+01$

$-.102102 u+01$

$.104 ? .4: 20+01$

$.1010650+01$

$.9793100+0)$

$.94847110+00$

- $5121200+00$

$.8382600+00$

$.8580000+00$

- $\$ 300.300+00$

-. (1) $16500+00$

$.7737800+00$

$.746 .3900+00$

$.7194900+00$

- $(430900+00$

- $671900+00$

$.6417700+00$

-t $1603500+30$

- $5924200+00$

$.5684300+03$

$.5450 \% 300+00^{\circ}$

$.5 \% 21000+(0$

$.4996500+01$

$.4776 .700+00$

$-.10(4.37)+01$

$-.1016) 10+01$

$-.9601 \ln 0+13$

$\cdots 7.710 n+00$

$-.37 \cdots .7300+00$

$-.8371700+01)$

$-.7360000+(3)$

$-.7562160+00$

$-.7187700+00$

$-.6806500+00$

$-.6448300+00$

$-.61) 3000+00$

$. .5770200+30$

$-.54497(2)+1)$

$-.51413(0)+00$

$-.4844700+01$

$-.4509 .300+00$

$. .42863(\div)+1)$

$-.402+200+(1)$

$-.7223730+00$

$-.7008600+00$

$-.3772500+06$

$-.35 .31 \therefore 10+12$

$-.6,01150.03+30$

$-.33019010+40$ 
. $22819 \mathrm{co}+00$

- .625? $400+100$ - $2061400+00$

$-.6129100+00$ - $1894400+00$

-.6004200+00

- $1720300+00$

-. $5877900+00$

- $1558500+0$

-.5750100+00

- $1408400+00$

$-.5620800+00$

- $1269400+C 0$

- .5493200+00

- $1141000+c 0$

$-.5358300+00$

- $1 \mathrm{c} 22600+\mathrm{c} 0$

-.52250c0+00

-9136000-01

-.5090400+00 - 8136000-01

- .4954000+00

- 72 $20000-01$

-.4817500+00

- $6384000-01$

-.4679300+00 . $5622000-01$

-.4539900+00 - 4431000-01

-.43943 Cu+00 - $4305000-01$

-.4257800+00 - $3742000-01$

-.4115100+00 -3235000-01

-.3971500+co

$.2782000-01$

- $38263 \mathrm{Cu}+00$ $.2378000-01$

-.3681200+0J -2020000-01

-. $3534700+00$ . $1704000-01$

- . $3337400+00$ $.1426000-01$

- .32.34200+00 - $1184000-01$

$-.3050200+00$ - $9740000-02$

-.2940400+u0 $.7730000-02$

$-.27839300+00$ . $63130000-02$

-. $2638700+00$ - $5030001-02$.

- .24 46900+00 - 339 c000-02

-.2334500+00
$-.1577100+00$ - $1000(0)+01$

-.1405900+00 - $1000000+01$

$-.1249800+00$ - $1000000+01$

$-.1107900+00$ - $1000000+01$

$-.9733000-01$ - $1000000+01$

$-.8628000-01$

. $1000000+01$

$-.7577000-01$

- $1000000+01$

- $6631000-01$

- $1000000+01$

$-.5782000-01$

- $1000000+01$

-.5023c00-01 - $1000000+01$

-.4345000-01 - $1000000+01$

$-.3743000-01$ - $1000000+01$

-.3209c00-01 - $1000000+01$

- 2739 c20-01 - $1000000+01$

- $\therefore 324000-01$ - $1000000+01$

$-.1902000-01$ - $1000000+01$

$-.16+6,000-01$

- $1000000+01$

$-.1372000-01$

- $1000000+01$

$-.1136000-01$

- $1700000+01$

$-.9340000-02$ . $1000000+01$

-.7610000-0z - $1000000+01$

$-.6160000-02$ $.1003000+01$

-.493000u-02 - $1100000+01$

- 3i1 u000-0a. - $10 \mathrm{coc} 0+01$

$-.3060000-02$ - $1000000+01$

-.2370000-0E: - $1000000+01$

- 1 \& $10 C(O)-02$ - $1000000+01$

$-.1300000-02$

- 1 J0000

-.1000000-0? - $1000000+01$
$-.6754400+00$

$.4562^{2}: 00+0 u$

$-.3(1)+16 \ldots+1)(1)$

$-.6557300+00$

$.435: 2500+00$

$. .2371500+c 0$

$-.6440300+30$

$.4147700+00$

$-.2571200+6.0$

$-.6283200+00$

$.3947800+00$

$-24 \div 95 n n+(0 i)$

$-.6126100+00$

$.3752000+00$

$-.2249100+00$

$-.5969000+00$

- J56ล $300+c 0$

$-.5811000+00$

$.3 \times 77900+0: 3$

$-.5654900+0)$

$.3197800+00$

$-.5497800+00$

$.3022(000+0)$

$-.5340700+00$

- $255233.00+00$

$-.5183000+00$

$-.5026500+00$

$. .48 t 3500+00$

$-.4712430+00$

$-.4555300+00$

$-.43982 u v+00$

$-.4241200+00$

$-.4084100+00$

$-.3927000+00$

$-.3769900+00$

$-.36128300+00$

$-.3455800+00$

$-.3298700+00$

$-.3141000+20$

$-.2984500+00$

$-.2827400+00$

$-.2670400+00$

$-.2513300+00$

$-.2356200+00$
$.2687000+00$

$.2526600+C \mathrm{C}$

$.2371200+00$

- $: 220700+00$

$.2075100+00$

- $1+34400+00$

$.1793700+00$

$.1663000+00$

$.1542100+00$

$.1421300+00$

$.1305300+00$

$.1194200+00$

$.1089100+00$

$.8907000-01$

- $7944(10) 0-0$ !

$.7131 \cup 0 u-01$

$.6317000-01$

$.5552000-01$
$.987,01) 0-01$
$-.2126717+10$

$-.1063200+00$

$-.1 \operatorname{son} 830 i+\div$

$-.15(.176)+$.

$-.1533 .300+(;)$

$-.13 .7 .300+20$

$-.12 .70000+00$

$-.11 \div .4000+00$

$-.1046 ! i l u+00$

$-.54 ; 3000-01$

-. $8508000-01$

$-.7620000-01$

$-.6812000-1) 1$

$-.6056000-01$

$-.53 .38 .00 .1 .01$

$-.4716000-111$

$-.41 ? 7: 100-01$

$-3(5: 4)(5) 00-01$

$-.31 r, 100.1-01$

$-.2058301001$

$-2260000-01$

$-.19) 4000-61$

$-.143000-11$

$. .1305000-01$ 
- 3080000-02

-.2181400+00 .2340000-02

-.2027900+00

$.1740000-02$

-. $1873800+00$

- 1260000-02

$-.1719300+00$

$.8900000-03$

-. $1564300+00$

$.6100000-03$

$-.1409000+00$

$.4000000-03$

- .1253300+00 $.2500000-03$

-. $1097300+10$

. 1500000-03

-.9411000-01 .8000000-04

-.7846000-01 . 4000000-04

-.0279000-01 . 2000000-04

-.4711000-01 . 0000000

-.3141000-01 .0000000

$-.1571000-01$

. 0000000

- 0030,300

.0000000

. 1571000-01

. 0000000

$.3141000-01$

.0000000

$.4711000-01$

.0000000

$.6279000-01$

$.2000000-04$

. $7846000-01$

$.4000000-04$

$.9411000-01$

. $8000000-34$

$.1097300+00$

- 1500000-03

. $1253300+00$

$.2500000-03$

$.1409000+00$

$.4000000-03$

$.1564300+00$

.6100000-03

$.1717300+00$

$.3900000-03$

$.1873300+00$

- $1260000-02$

$.2027900+00$

$.1740000-02$

$.2181400+00$
$-.7300000-0.3$

- $1000000+01$

$-.5100000-03$

. $1000000+01$

$-.3600000-0 z$

- $1000000+01$

$-.2400000-03$

. $1000000+01$

$-.1500000-03$

$.1000000+01$

$-.1000000-03$

$.1000000+01$

$-.6000000-04$

- $1000000+01$

-.3000000-04

- $1000000+01$

-.2000000-04

$.1000000+J 1$

-.1000000-04

- $1000000+01$

- 0000000

- $1000000+01$

- cccocoo

- 1000 nuvitos

. 0000000

$.1000000+01$

- CC00000

- $1000000+01$

- 0000000

- $1000000+J 1$

- 0000000

$.1000000+01$

.0000000

$.1000000+01$

- 0000000

- $1000000+01$

.0000000

$.1000000+01$

- $\operatorname{cocc000}$

- $1000000+01$

- c000000

$.1000000+01$

. $1000000-04$

$.1000000+01$

. 20c0c00-04

. $1000000+01$

- 30c0000-04

- $1000000+01$

.60 Cu0v0-04

. $1000000+01$

$.1000000-03$

- $1000000+01$

- $1500000-03$

$.10000(1)+01$

. 2400000-03

$.1000000+01$

$.3600000-03$

$.1000000+01$

$$
-.2199100+30
$$

$-.2042000+00$

$-.1885000+00$

$-.1727900+00$

$-.1570800+00$

$-.1413700+00$

$-.1256600+00$

$-.10 \times 9600+00$

$-.9425000-01$

$-.7854000-01$

$-.6283000-01$

$-.47120100-01$

$-.3142000-01$

$-.1571000-01$

.0000000

$.1571000-01$

$.3142000-01$

$.4712000-01$

$.62830 .00-01$

$.7854(00-)$.

$.9425000-01$

$.1099600+00$

$.12560,00+30$

$.141 .3700+00$

$.1570800+00$

$.1727900+00$

$.1885000+00$

$.2042000+00$

$.2199100+00$
$.4(4.3(-3))-01$

$.4170000-01$

$.3553000-01$

$.2986000-01$

$.2467000-01$

$.1999000-01$

$.1579000-01$

$.1209000-01$

$.8880000-02$

$.0170000-0 ?$

$.3050000-02$

$.2220000-02$

$.9900000-03$

$.2500000-03$

.0000000

$.2500000-03$

$.9900000-03$

. $2223000-02$

. 3050000-02

$.6170 n 00-02$

$.8880000-02$

$.1209000-01$

$.1579000-01$

$.1999000-01$

$.2467000-01$

$.2536000-01$

.3553000001

$.4170000-01$

$.4830000-31$
$-.10(4+11)-71$

$-.3520000-0 ?$

$-.076) \div 010-02$

$-.5160700-112$

$-.38 \leqslant 000)-02$

$-.2830000-02$

$-.1930 .000-02$

$-1.130690-22$

$-.4400)(0-0.3$

$0.4300000-03$

$-.2500000-43$

$-.1000 .0 .03$

$-.30010000-1.4$

.3000000

.00010000

.0030100

$.3000000-04$

- $1002703-03$

$.2500000-0.3$

$.48 .20000-03$

$.8400000-03$

$.1330000-02$

- $(931) 00 n-1) ?$

- $2330000-02$

$.3882000-02$

. 319005 (1-002

$.6700000-02$

$.35 a 0000-02$

$.1004000-31$ 
.2340000-02

$.2334500+00$

. 3080000-02

$.2486900+00$

. 399 C000-02

. $2638700+00$

. 5080000-02

$.2789900+00$

.6390000-02

. $2940400+00$

- 7930000-02

$.3090200+00$

. 9740000-02

- $3239200+00$

. $1184000-01$

. $3387400+00$

$.1426000-01$

. $3534700+00$

. $1704000-01$

- $3681200+00$

. 2020000-01

. $3826300+00$

.2378000-01

$.3971500+00$

$.2782000-01$

. $4115100+00$

. 3235000-01

$.4257800+00$

.3742000-01

$.4359400+00$

$.4306000-01$

$.4535 ; 00+00$

$.4931000-01$

$.4679300+00$

. 562:2000-01

$.4817500+00$

$.6384000-01$

$.4954600+00$

$.7220000-01$

$.5090400+00$

. $0130000-01$

. $5225000+00$

. 9136ग00-01

. $5358300+00$

- $1022500+00$

$.5490200+00$

$.1141000+00$

. $5620800+00$

$.1269400+00$

$.5750120+00$

$.1408400+00$

$.5 \times 77110+0$ ?

- $1558500+00$

$.6004200+30$

. $1720300+60$

. $6129100+00$

- $18,4400+00$

-vá $24000+00$
$.5100000-03$

$.1000000+01$

$.7300 \mathrm{cos}-03$

- $1000000+01$

- $1000000-02$

- $1000000+01$

- $1360000-02$

- $1000000+01$

- $1810000-02$

. $1000000+01$

. 2370c00-02

$.1000000+01$

. 3060000-02

$.1000000+01$

.3910000-02

$.1000000+01$

. $4730000-02$

. $1000000+01$

- E160000-02

- $1000000+01$

- 7010000-02

- $1000000+01$

$.9340000-02$

- 10 c0unotol

- $1136000-01$

- $1000000+01$

- $1372000-01$

- $10.00000+01$

- 1640000-01

- $1000000+01$

- $1962000-01$

$.1000000+01$

$.2324000-01$

$.1000000+01$

$.2738000-01$

$.1000000+01$

- 3200 coo-01

$.1000000+01$

$.3743000-01$

. $1000000+01$

$.4345000-01$

$.1000000+01$

$.502: 3000-01$

$.1000000+01$

$.5782(00-1) 1$

$.1000000+01$

$.6631000-01$

- $1000000+01$

$.7577000-01$

- $1000000+01$

. 8628c00-01

- $1000000+01$

$.97+3600-01$

$.1000000+01$

- $1107900+00$

- 1000000+01

$.1249800+00$

$.100000 u+J 1$
$.2356200+00$

$.5552000-01$

$.1308000-01$

. $2513300+00$

$.6317000-01$

$.1583000-01$

$.2670400+00$

$.7131000-01$

$.2827400+00$

$.7994000-01$

. $2984500+00$

$.8907000-01$

$.3141600+00$

$.9870000-01$

$.3293700+00$

- $1088100+00$

$.3455800+00$

$.3612900+00$

$.3769900+00$

$.3927020+00$

$.4084100+00$

$.4241200+00$

$.4398200+00$

$.4555300+00$

$.4712400+00$

. $48695^{\circ} 00+00$

$.5026300+00$

$.518360 u+00$

$.5340700+00$

$.5497800+00$

$.5654900+00$

$.5811900+00$

$.5969000+00$

$.6126100+00$

. $283320.1+0 \mathrm{~J}$

$.6440 j 00+00$

$.6597300+00$

$.6754400+00$
$.1194200+00$

$.1305300+00$

$.1421200+00$

$.1542100+00$

- LE $63300+00$

$.1748700+00$

$.1934400+00$

$.2075100+00$

$: .2220700+00$

$.2371200+00$

$.2526600+00$

$.2687000+100$

$.2852300+00$

$.3022000+00$

$.3197800+00$

$.3377900+00$

$.3562000+00$

$.375 \% 900+00$

$.394780 .3+00$

$.41477(11)+(10$

$.4352500+00$

$.4502200+00$
$.1904000-01$

$.2260000-01$

. 26.580 0-01

$.3101000-01$

$.3589000-01$

$.41270010-01$

$.4716000-21$

$. \$ 3558000-01$

$.5056000-01$

$.6312000-01$

$.7629000-01$

$.3508000-01$

. $5453000-01$

$.1046500+00$

$.1154000+00$

-12.70000+(30

$.13025300+00$

$.1523300+20$

$.16 t 1700+00$

$.1308 .300+00$

- $1403200+00$

- $21 \geq n>n 0+0$ y

$.2290100+00$

$.243750+30$

$.2671200+10$

$.2371500+00$

$.30 \times 1300+c 0$ 
- $\angle C 31400+()$ - :374200+? - $22319 \mathrm{CO}+00$ . $ن 494500+0$ ? . $2496500+00$ . $5613102+01$ . $2725900+00$ . $6730100+00$ - $2970800+00$ - $6845500+00$ - $32318 \mathrm{CO}+00$ . $5959100+00$ $.3509700+00$ $.7071100+00$ - $3805000+00$ - $7181300+00$ - $4118700+00$ - $7289700+00$ - $4451400+00$ . $7396300+00$ $.48038 \mathrm{CO}+00$ - $7501100+00$ $.5176700+0$ ? $.7604100+00$ $. \$ 571000+00$ . $7705100+00$ $.5957300+00$ . $7804300+00$ . $6426600+00$ $.7901600+00$ . $6889600+00$ . $7996800+00$ $.7377100+00$ $.8090200+00$ . $7890101+00$ - 3131500+00 - $3429400+00$ - $2270800+00$ - $8995900+00$ . $8358100+00$ . $9590500+00$ . $3443300+00$ - $1021410+01$ $.8526400+00$ - $1086760+01$ $.8607400+00$ $.1155190+01$ - $4696300+00$ - $1226810+01$ - $8703100+00$ - $1301710+01$ - $8837700+00$ $.137 \rightarrow 790+01$ $.5910100+00$ - $1461750+01$ . $39 \cdot 30300+00$ - $1547080+01$ $.4043300+00$
- $140590 .+00$ - $1000000+01$ -157710J+00 . $1000000+01$ - $17647 \mathrm{CO}+0 \mathrm{O}$ - $1000000+01$

- $1969600+00$

- $1000000+01$

- $2193300+00$

$\therefore 1000000+01$

- $2435700+00$

- $1000000+01$

$.27013 \mathrm{CO}+00$

- $1000000+01$

- $2988500+00$

- $1000000+01$

- $3299500+00$

$.1000000+01$

- $3635900+00$

- $1000000+01$

- 3599300+00

- $100000 J+01$

. $43510 u j+i j u$

- $1000000+01$

- $4313 \mathrm{CJO}+0 \mathrm{~J}$

- $1000000+01$

. 5260700+00

- $1000000+01$

. $5754100+00$

- $1000000+01$

. $6276800+00$

- $1000000+01$

- $6836900+00$

- $1000000+01$

$.7436300+00$

- $1000000+01$

. $8077000+00$

- $1000000+01$

. $8761100+00$

- $1000000+01$

- $9490800+00$

. $1000000+01$

- $1026830+01$

- $1000000+01$

- $11096 \mathrm{~J} 0+01$

- $1000000+01$

- $1197620+01$

- 1)00000+01

- $1291140+01$

- $1000000+01$

- $1390410+01$

- $1000000+01$

- $1495700+01$

- $1000000+01$

- $1607270+01$

- $1000000+01$

- $1725410+01$

- $1000000+01$
$.5911500+00$

$.477 ; 900+00$

$.3201500+00$

$.7068000+00 \quad .4996500+0.3$

$.3531300+0.7$

$.7223700+00$

$.5221000+00$

$.3772300+00$

$.7382700+00$

$.5450500+00$

$.4024000+C 0$

$.7539300+00$

$.5684900+100$

$.4286 .300+00$

$.7696400+00$

$.5924200+00$

$.4559800+01$

$.7354000+00$

$.6163500+00$

- $+844730+00$

$.3011100+00$

$.6417760+00$

$.5141300+00$

. $9168100+00$

$.6671900+00$

$.9325200+00$

. $6930900+00$

$.84 r^{2} ? 300+00$

$.7194900+00$

$.863 .3400+00$

$.7463900+00$

$.57: 56500+00$

$.7737800+00$

- $8953500+00$

$.8016600+00$

$.91106,00+00$

$.8300300+00$

$.9267700+00$

$.8589000+00$

$.9424800+00$

$.8 \varepsilon 82.600+0 u$

$.9581900+30$

$.9181200+00$

$.9738700+00$

$.98: 36000+00$

- $1005310+01$

$.1021020+01$

$.1036730+01$

$.1052430+01$

$.1068140+01$

$.1083850+01$

$.1049500+01$

$.1115270+01$

$.1130970+01$
$.9484700+00$

$.9793100+00$

$.1010650+01$

$.1042480+01$

$.1074800+01$

$.1107 E 20+01$

$.1140930+01$

$.1174730+01$

$.1209030+01$

- $1243820+01$

$.1279100+01$
$.5440700+.3$

$.5770 .30(1+50$

$.6103000+00$

- $6448300+00$

$.6806500+00$

$.7177700+00$

$.7552100+00$

$.7360000+00$

$.8371700+00$

$.37 ? 7300+(9)$

$.92371 n \%+n 0$

$.9691 .30 n+00$

$.1016010+? 1$

$.1064390+01$

$.1114 ? 7.1+1$

$.1165697+01$

$.121867 n+31$

$.1273230+01$

$.1329300+01$

$.1 .387190+01$

$.144 E E 3()+01$ 
. $1636100+01$

- $9114000+00$

- $1728900+01$

$.9177500+00$

- $1825600+01$

- $9238800+00$

- $1926300+01$

$.9297800+00$

. $2031110+01$

$.9354400+00$

$.2140140+01$

$.9408800+00$

- $2253500+01$

- $9460900+00$

. $2371310+01$

$.9510500+00$

$.2493670+01$

$.9557900+00$

. $2620710+01$

$.9602300+00$

$.2752550+01$

- $7645600+00$

. $2889290+01$

. $9685300+00$

. $3031070+01$

. $3723700+00$

$.3178010+c 1$

$.9759200+00$

. $3330220+01$

$.9792200+C 0$

. $3487840+01$

. $9322900+00$

$.3650990+01$

$.9851100+00$

$.3819790+01$

- $9876900+00$

. $3994380+01$

$.9900200+00$

$.4174890+01$

$.9921100+00$

$.4361450+01$

$.9939600+00$

$.4554190+01$

$.9555600+00$

$.4753250+01$

$.9969200+00$

$.4958770+01$

- $9430300+00$

. $5170830+01$

. $3988900+00$

. $5389720+01$

. $9995100+00$

$.5615440+01$

. $9988800+00$

$.5848170+01$

$.100000 n+01$

$.6083070+01$

. 3000000
$.1850380+01$

$.1000000+01$

$.1982500+01$

- $1000000+01$

. ? $122060+01$

- $1000000+01$

. $2269370+01$

$.1000000+01$

$.2424750+01$

. $1000000+01$

$.2583530+01$

. $1000000+01$

- $2761040+01$

. $1000000+01$

$.2942620+01$

- $1000000+01$

$.3133640+01$

- $1000000+01$

- $3334450+01$

- $1000000+01$

$.3545430+01$

. $1000000+01$

$.3766950+01$

- $1000000+01$

- $3903410+01$

$.1000000+01$

$.4243200+01$

- $1000000+01$

.44 SE $750+01$

- $1000000+01$

$.4766460+01$

- $1000000+01$

$.5046760+01$

- $1000000+01$

- $5340100+01$

- $1000 c 00+01$

. $5646920+01$

$.1000000+01$

$.5967690+01$

$.1000000+01$

$.6302870+01$

$.1000000+01$

$.6652950+01$

. $1000000+01$

$.7018410+01$

$.1000000+01$

$.7354760+01$

- $1300000+01$

$.7797500+01$

$.1000000+01$

$.8212170+01$

$.1000000+01$

. $304430 J+01$

- $1000000+01$

- $9094430+01$

- $1000000+01$

$.9563120+01$

.0000000
$.1146680+01$

$.1314830+01$

$.1507750+01$

$.1162390+01$

- $1351150+01$

$.15 .7 .0500+01$

$.1173100+01$

- $1337910+01$

$.1035100+01$

$.1153810+01$

$.1425170+01$

$.17013330+01$

$.1209510+01$

$.1462920+01$

$.1763420+01$

$.1225220+01$

. 15$) 1170+01$

$.1830260+11$

. $1240930+01$

$.1539910+01$

$.1910910+01$

$.1256640+01$

$.1579140+01$

$.190440 u+u 1$

$.1272350+01$

$.161 \times 850^{\circ}+01$

$.2059753+01$

$.1288050+01$

$.16590: 30+01$

. $213(0,0 \div 3)+01$

$.1303760+01$

$.1699790+01$

...21 $21 \geq 0+01$

$.1310473+i 1$

$.1741000+01$

$\left..229710_{0}\right)+01$

$.13351: 0+01$

$.1782700+01$

$.238022(i+31$

$.1350480+01$

$.1824390+01$

$.2465220+01$

$.1366530+01$

$.1667580+01$

$.2552 ? \because 0+01$

$.1382300+01$

$.1918750+01$

$.204124 u+01$

$.1393010+01$

$.1954430+01$

- $27323310+01$

$.1413720+01$

$.1398 \div 3 \div 0+01$

$.2335450+01$

$.1429420+01$

$.2043250+01$

$.2920080+11$

$.1445130+01$

$.2088410+01$

$.3018050+01$

$.1460840+01$

$.21340: 0+01$

$.3117510+01$

$.1476550+01$

$.2180200+01$

$.3219180+01$

$.1492260+01$

$.2226830+01$

- $3323000+01$

$.1507960+01$

$.2275900+01$

. 342:050 +01

$.15236,7.7+0$

$.2321580+01$

$.1539380+01$

$.2369(091)+01$

- $353732 \cdot 3+01$

$.3647 \varepsilon 61+01$

$.15550 .00+01$

$.241+300+51$

$.37(0.067 i)+01$

- $1570300+01$

$.2407400+01$

$.3875780+01$

$.1000000+04$

$.7402 .203+04$ 
$-.1550314+22 \quad .304433+405$ . 0000000

$.1550314+.55$
- 10600.10+04

- $000(001)$

. $3044034+05$
. $314 i: 3-1+i) 4$

$.74(12393+044$ 
HP21 : TRIGONOMETRIC AFPROXIMATION PRCILEM

2111

- $1000000+01$

$.1000000+01$

.0000000

- $10.51270+01$

- $9950000+00$

$.2474000+00$

- $1105170+01$

- $3800700+00$

- $4794300+00$

- $1161830+01$

- $9553400+00$

- $6816400+00$

- $1221400+u 1$

- $9210600+00$

. $3414700+00$

- $1284030+01$

$.8775800+00$

$.9489800+00$

- $1349860+01$

- $3253400+00$

- $9974900+00$

$.1419370+01$

. $7648400+00$

- $93399 \mathrm{C}(1)+.70$

$.1491820+u 1$

$.6767100+10$

. $9093000+00$

- $1568310+01$

- $6216102+00$

. $7780700+00$

- $1 \epsilon 48720+01$

$.5403000+00$

- $59847 \mathrm{CO}+00$

- $1648720+01$

- 4536$) \mathrm{Co+00}$

$.381 .6600+00$

- $1648720+01$

- $3 E 2.3600+00$

- $1411200+00$

$.1648720+01$

- $2675000+00$

-. $1082000+00$

- $164 \times 3720+01$

- 1:99700+00

$-.3507400+00$

- 164 ć $720+01$

$.7074000-01$

$-.57156 \mathrm{cc}+00$

$.1645720+01$

-.2920000-01

- 7563 C Cu+0o

- 1 is 4 is $720+01$

- $1283400+00$

-. $3949300+10$

$.164837 ? .0+01$
$.1000000+01$

. 0000000

- $1000000+01$

- $1000000+01$

- $1494400+00$

$.9689100+00$

- $1000000+01$

- $2955200+00$

. $3775800+00$

- $1000000+01$

. $4343700+00$

. $7316900+00$

- $1000000+01$

. $5646400+00$

$.5403(0)+00$

$.1000000+01$

- $6816400+00$

- $3153200+00$

- $1000000+01$

$.7833300+00$

$.7074000-01$

- $1000000+01$

- $86742 u v+00$

-. $1782500+0 J$

- $1000000+01$

- $9320400+00$

-.4161500+00

- $1000000+01$

- $9757200+00$

-. $231700+00$

- $1000000+01$

- $9974: 00+00$

-.8011400+00

- $1000000+01$

- $9963700+00$

-.9243000+00

- $1000000+01$

- $7738500+00$

-. $5559900+00$

- $1000000+01$

- $9289600+0$ 0

-.9941300+00

- $1000000+01$

- $8632100+00$

$-.03646100+03$

- $1000000+01$

$.7780700+00$

$-.82055(0+0)$

- $1000000+01$

$.6754600+0$ I

$-.6536400+00$

- $1000000+01$

. $5576800+c 0$

$-.44(20900+0)$

$.1000000+01$
- 0000000

- 1000 rootol

- $4938000-01$

- $9887700+00$

- $9983000-01$

$.9553400+00$

$.1494700+00$

- $9004500+00$

$.1986700+00$

. $825.3400+00$

$.2474000+00$

- $7316900+00$

$.2955200+10$

$.6216100+00$

- $3429001+00$

$.4975700+00$

- $3894200+00$

. $3623600+00$

- $4349700+00$

$.2170100+00$

- $4794300+00$

$.7074000-01$

- $5226900+00$

-.7912000-01

$.5646400+00$

$-.2272000+00$

- $6051900+00$

$-.3701800+00$

$.0442 .200+100$

$-.5048500+00$

$.6816400+00$

$-.6281700+00$

$.7173600+00$

$-.737 .390 \%+00$

. $7512300+00$

-. $3300500+00$

$.7835300+00$
- $1000000+01$

- Oc00000

- $9987500+00$

- $1986700+00$

- $9950000+00$

$.3 .394200+00$

.7887700+01)

- $564 j 400+00$

$.9800700+00$

$.7173600+00$

- $968 \geqslant 100+00$

. $8414700+00$

- $9553400+00$

- $9320400+00$

$.930 .3700+00$

$.9854500+00$

- $9210600+00$

. 9995700+00

- $9004500+00$

- $978500+00$

$.8775800+00$

$.90 .350 .10+100$

$.8525200+00$

- $8.385000+00$

$.9253400+00$

$.6754600+00$

$.79508(1)+0()$ . $5155000+00$

$.7648400+00$

. $3344,900+00$

$.7316900+00$

$.1411: 30+00$

- $64(7100+1) 0$

$-.5\{37) 0)-01$

$.6599800+00$

$-.2555400+00$

$.6216100+00$
- 0000060

- 1 i. $00000+01$

- 9y $3000-01$

$.980070 \mathrm{C}+00$

$.14867: 10+j J$

$.9210 \in 0 i+00$

- $2455: \dot{U} 0+0 \dot{0}$

. $4253400+00$

- 3894200+00

$.6767100+00$

$.47 c .4310+00$

. $5403000+(0)$

- $5346400+00$

- 3t. $3600+10$

- $6442.210+00$ - 16 व $7700+00$

$.71736,00+00$

-.2920000-01

- 7833300+00

$-.2272 .000+00$

. $3414703+00$

$-.41(150)+00$

- $871 \% .100+00$

- . 5885.)0 000

. $3320410+00$ -.7373900+00

- Se $35 \div 100+00$

-.8568.700+00

$.9854300+00$

-. $94 \because 2200+00$

- $997+90 h+00$

- $98+x, 700+00$

$-\therefore(2,370)+00$

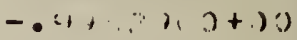

- $9916000+00$

- . gérisocotoo

$.07 .38500+00$ 
$-.2272000+00$

$-.9775300+00$ $.1643720+01$

$-.3232900+00$

-.9992900+00 - $1648720+01$

-.4161500+00

-. $9589200+00$
. $4273800+00$

$-.2108000+00$

$.1000 \mathrm{COO}+01$

- $28748 \mathrm{CO}+00$

. 37E0000-01

$.1000000+01$

- $1411200+00$

- 28.36600+00

$$
\begin{array}{rrr}
-.9040700+00 & -.4425200+00 & -.8967110+00 \\
.8134200+00 & .5816300+00 & .9463000+00 \\
-.9577400+00 & -.6118500+00 & -.7907700+00 \\
& & \\
-.8414700+00 & .5403000+00 & .9033000+00 \\
-.9399900+00 & -.7568000+00 & -.6536400+00
\end{array}
$$


HP22: TRIGCNCVITRIC. APPROXIMATION PR:3:.EM

$51 \quad 11$

$1000000+01$

- $1000000+01$

.0000000

- $1020200+01$

$.9992000+00$

- $7983000-01$

. $1040810+01$

$.9968000+00$

- $1986700+00$

: $1061840+01$

. $9928100+00$

: $2955200+00$

- $108.3290+01$

. $9872.300+00$

- $3894200+00$

$.1105170+01$

- $800700+00$

$.4794300+00$

. $1127500+01$

- $9.713400+00$

$.56464(0+) i)$

- $1150270+01$

- $9610600+00$

. $6442200+00$

$.1173510+01$

. $9492400+00$

$.7173600+00$

- $1197220+01$

. $359000+00$

. $7833300+00$

- $1221400+01$

- $9219600+00$

- $3414700+00$

- $1246080+01$

- $9047500+00$

- $8912100+00$

$.1271250+01$

- $3869900+00$

- $4320400+00$

$.1296930+01$

- $86783200+00$

- $96.35600+00$

- $1.323130+01$

- $3472000+00$

- $9854500+00$

- $1.349860+01$

. 825340$)+00$

- $9974900+00$

- $1377130+01$

- $3021000+40$

$.9995700+00$

$.1404 .150+01$

$.7775700+00$

- $9916600+00$

$.1433330+01$
$.1000000+01$

.0000000

- $1000000+01$

- $1000000+01$

-5996000-01

- $9950000+00$

- $1000000+01$

- $11971(0+01)$

. $9800700+00$

$.1000000+01$

- $1730300+00$

- $9553400+00$

- $1000000+01$

- $2377000+00$

$.92106 \mathrm{CJ}+\mathrm{Cu}$

- $1300000+01$

- $2955200+00$

- $3775360+0 u$

- $1000000+01$

- $3522700+00$

- $8253400+00$

- $1000000+01$

. $4077600+00$

. $76423400+0 u$

- $1000000+01$

. $46178 \mathrm{CO}+00$

. $6967100+00$

- $1000000+01$

- $5141400+00$

. $6216102+00$

- $100000 u+01$

- $5646400+00$

. $5403000+00$

- $1200000+01$

- $6131200+00$

- $4535000+00$

- $1000000+01$

$.6593800+00$

- $3623600+00$

- $1000000+01$

. $70328 \mathrm{CU}+00$

. $2675000+00$

- $1000000+01$

. $7446400+00$

- $1699700+00$

- 1) $00000+01$

. $783330 u+00$

. 7074000-01

. $1000000+01$

- 3191901$)+00$

$-.2320000-01$

- $1000000+01$

. $8521100+00$

$-.1288400+00$

- $1000600+01$
- 0c00000

- $1000000+01$

$.1000000+01$

- 0000000

- $9498000+00$

- 2000000-01

. $7991000-01$

- $9982000+00$

- 3999000-01

- $9992000+00$

- $9928100+90$

- $1593200+00$

. 5996000-01

- $9982000+00$

- $9838400+00$

- $799103 n-01$

. $9713400+00$

. $598.3000-01$

. $9553400+00$

- $1197100+00$

- $9359000+00$

- $1395400+00$

- $9130900+00$

$-1593200+00$

$.8369900+00$

$.1790300+00$

$.8577100+00$

- $1986700+00$

- $8253400+00$

$.2182300+00$.

- $7894300+00$

$.2377000+00$.

$.7518100+00$

. $25708300+00$

$.71041 .00+00$

- $2703600+00$

. $6674600+00$

- $2955200+00$

- $6216100+(10$

$.3145700+00$

$.57 .352(0)+00$

- $3334900+00$

. $52.33700+00$

$.352 .2700+00$
$.2377000+00$

- $996,8000+00$

$.3145700+00$

- $9350000+00$

.389421) 0+00

- $942810.0+00$

$.4617800+00$

- 9902200+00

$.5 .311900+00$

$.9372300+00$

$.5972000+00$

$.9838400+00$

$.6593800+00$

$.9300700+00$

$.7173600+00$

$.9759000+00$

$.7707400+00$

$.97134(00+0 n$

- $8191900+00$

- 96.63900+00

. $8624000+00$

- $9610 t, 00+00$

- $\rightarrow 00100$ + 100

. $955.3400+00$

- $9320400+100$

- $9492400+00$

- $95.30: 00+00$

$.9427500+00$

. $9778600+00$

$.935(1000+0) 0$
. 0000000

$.10030 ! 0+31$

- 3999 or $0-01$

- syta $3000+00$

- 7991 ::0-11

$.48723 j+00$

$.1197100+00$ $.9713400+00$

- $156,3200+1 ; 0$ $.949240(1+0)$

$.1945700+00$ . $9210000+00$

- $237700 \div+00$ - $\varepsilon .364300+00$

- $2763000+00$ . $8472500+130$

$.3145700+00^{\circ}$

$.8021000+00$

. $3522700+00$

$.7513100+00$

- $3894200+00$ . $6,967100+00$

-4254400+(1) - $(3715 \mathrm{CO}+1) 0$

- 4i317:101)+(0) - $5735200+v u$

- $45630300+00$ - 51)6??.)n+60

$.5311092+11$ . $4356300+i n$

$.5646420+00$

- $\left.36 z^{2} \sin (x)+1\right) 0$

- $5072000+0$

- $38 \div 7500+10$

. $6 ? 47700+00$ . $204 ? 400+00$

- $6533500+0$ 
$.7518100+00$ . $9738500+00$ - $146.2280+01$ $.7248 i 400+00$ . $9463000+00$ $.1491820+01$ $.6967100+00$ - $9093000+00$ - $1521960+01$ $.6674600+00$ $.8632100+c 0$ $.1552710+01$ . $6371500+00$ $.8085000+00$ $.15841) 70+01$ $.5058200+00$ $.7457100+00$ . $1616070+01$ $.5735200+00$ . $5754<00+00$ - $1644720+01$ $.5403000+00$ . $5984703+00$ . $1648720+01$ $.5062200+00$ $.5155000+00$ $.1648720+01$ $.4713300+00$ $.427 .3300+00$ - $1643720+01$ . $4356300+00$ . $33499 \mathrm{CO}+00$ $.1548720+01$ . $3993400+C, 0$ $.2392500+00$ - $1648720+01$ - $3623600+00$ $.1411200+00$ . $1648720+01$ $.3248000+00$ .4158000-01 $.1648720+01$ - $2967200+00$ -.5837000-01. - $1643720+01$ . $2481800+00$ $-.1577500+00$ . $1643720+01$ . $2092400+00$ $.2555400+00$ $.1648720+01$ $.1697700+0$ ) -. $.507800+00$ - $1543720+01$ . $1304200+00$ -. .4425200+00 - $1648720+01$ - $7067000-01$
- $88196(11)+00$

$-.2272000+00$

- $1000000+01$

$.9086300+00$

-.3232900+00 . $1000000+01$ $.320400+00$

-.4161500+00 . $1000000+01$ . $5520900+00$

-.5048500+00 . $1000000+01$ $.9687200+00$

$-.5885000+00$ - $1000000+01$ . $9818500+00$

$-.6662800+00$ . $1000000+01$ $.9914600+00$

-.7373500+00 . $1000000+01$ $.9974900+00$

$-.8011400+00$ $.1000000+01$ $.9999400+00$

-.8568500+00 - $1000000+01$ $.3937300+00$

-. $9040700+00$ - $1000000+01$ - $9940400+00$

-.9422200+00 . $1000000+01$ - $98572 \mathrm{CO}+00$

$-.9709600+00$ $.1000000+01$ - $3738500+00$

-. 9 तs $900+00$ - $1000000+01$ - $9584700+00$

-.9991400+00 . $1000000+01$ - $9396500+00$

-. $5982900+00$ - $1000000+01$ - $\leqslant 174400+00$

-.9874800+00

$.1000000+01$

- $9913300+00$

- .9c630iva 00 $.1000000+u 1$ - $8632100+00$

- . 3 $364 \epsilon_{(} D+0 u$

- $100000 J+01$

. $83138 \mathrm{co}+0 \mathrm{O}$

-. $\varepsilon \$ 67600+00$

- $1000000+01$

- $746.5700+00$
$.4713300+00$

$.3709200+00$

$.4175400+00$

$.3894200+00$

$.3623600+00$

$.4077600+00$

$.3058200+00$

$.4259400+00$

. $2481500+00$

$.4439500+00$

- $1896400+00$

$.4617800+00$

$.1304200+00$

$.4794300+00$

$.7074000-01$

$.4968800+00$ $.1080000-01$

$.5141400+00$ -.4918000-01

$.5311900+00$ -. $1089900+00$

$.5480200+00$ -.1684000+00

$.5646400+00$ $-.2272000+00$

. $5810400+00$ $-.2851900+00$

$.5972000+00$ $-.3421500+00$

$.6131203+00$ -.39788ro+oo

$.6287900+00$ $-.4521300+00$

$.6442200+00$ $-.5043500+0)$

. $0593800+00$

$-.5557000+00$

$.6742900+00$

$-.6045500+00$
- $9014000+00$

- $9283.000+c 0$

$.9387100+C 0$

. $9210600+00$ $.9995700+00$

$.9130700+00$ $.9940400+00$

$.9047500+00$ $.9821500+00$

. $3960500+03$ . $98.39800+00$

$.8869900^{\circ *}+00$ $.9396 .500+0.3$

. $8775800+00$ $.9053000+00$

$.8678200+00$ . $8731300+00$

$.8577100+00$ $.3313800+00$

$.8472600+00$ $.7843200+00$

- $6364000+00$ $.7322300+00$

. $8252400+00$ $.6754000+00$

$.8133800+00$ $.6143700+00$

. $8021000+00$ $.5453600+00$

. $7899400+00$ $.4808200+00$

$.7775700+00$ $.4092100+00$

$.7648400+00$ $.3 .34 \% \cdot 100+00$

.751 cilootoo $.2536 .200+u 0$

$.7384700+00$ $.1306000+00$
$1.304200+00$

-

-5077000-01

$.71736,00+00$ $-.2520000-01$

$.7446400+00$

-.1089900+00

$.7707400+00$

$-1883800+00$

- $7956000+00$

-. 26.5960 0+00

$.815140,+00$

$-.34 ? 1500+00$

$.8414700+00$

$-.4161300+00$

- $36.24000+00$

$-.4874300+00$

- $83196.00+00$

$-.5557(0)+00$

- $9001000+00$

- E?OJE00 + UO

- $916: 3000+00$

$-.0310600+00$

- $320400+00$

-. $7373900+00$

$.9457300+00$

$-.7890100+00$

- 9530200700 -.835:5900+00

- $963 ? 200+00$

$-.876 .3200+00$

$.0778500+00$

$-.9124401+13$

$\therefore 9854500+00$

- 942:?

$.901+500+00$

- . 9 Es: ju+00

- .9958001$)+00$

$-.9835600+00$ 
$-.5298400+00$ - $164.5720+01$ $.5077000-01$

$-.0118600+00$ $.1648720+01$ . $1080000-01$

$-.6877700+00$ . $1648720+01$

-.2920000-01

-.7568000+00 $.1645720+01$

-.6915000-01

$-.3182800+00$ . $1648720+01$

- $1089900+00$

-. $3715600+00$ $.1648720+01$

-.1486500+00

$-.9161700+00$ - $16,48720+01$

-. $1080800+00$

-. $4516000+00$ - $1648720+01$

-. $2272000+00$

$-.9775300+00$ . $1648720+01$

-.2659600+00

- .9936900+00 $.1648720+01$

-. $3043000+00$

-. $9999200+00$ - 16:40720+01

$-.3421500+00$

-.996160J+00 $.1648720+01$

$-.3794500+00$

- .9824500+00 - $1048720+01$ -.4161500+00 -.9583200+00
$-.3481000+00$

$.1000000+01$

$.1588800+00$

$-.7909700+00$

$.1000000+01$

$.7184600+00$

$-.7259300+00$ . $1000000+01$ . $6754600+01$

$-.6535400+00$ . $1000000+01$ - $6300300+00$

$-.5748200+\mathrm{CO}$ - $1000000+01$ . $5823300+00$

-.4902600+00 . $1000000+01$ $.5325300+00$

$-.4008000+00$ - $1000000+01$ $.43082 \mathrm{CD}+00$

-. $30733 c^{\circ} 0+00$ $.1000000+01$ $.4273800+00$

$-.2108000+00$ . $1000000+01$ - $3724000+00$

-.1121500+00 - $1000000+01$ $.31608110+00$

-.1239000-01 - $1000000+01$ $.2586200+00$ - 8753000-01 . $1000000+01$ $.2002300+00$ - $1365100+0 u$ - $1000000+01$ $.1411200+00$ . $2336600+00$
$.6889200+00$

$-.65123 .20+30$

. $7032300+00$

$-.6955600+00$

$.7173600+00$

-. $7373200+00$

. $7311500+00$

$-.7765700+00$

- $7446400+00$ $-.8129500+00$

$.7578400+00$ -.8464100+00

$.7707400+00$ $-.876 .9200+00$

- $7833300+00$

-. $9040700+00$

. $7956000+00$ $-.9280700+00$

$.8075600+00$ - $9487300+00$

- $8191900+00$ $-.9659800+00$

- $83050: 00+00$ $-.9797500+00$ :

$.8414700+00$ $-.9859900+00$
$.7248400+00$

- $101: 2: 60+0$ ?

$.7100100+00$

$.2159: 000-01$

. $6467100+00$

-.5837000-01

. $6822200+00$

$-.1370700+00$

- $0674000+(-)$

$-.2166300+00$

$.6524400+00$

$-.2940000+0$ )

. $6371500+00$

$-.36: 944 c c+00$

$.6210100+00$

$-.4425200+00$

. $6058200+00$

$-.5127700+00$

. $5897903+00$

-. $5797400+00$

. 5735201$)+00$

-.6430000+6io

$.5570200+00$

-.702152. +0u

$.54030010+00$

$-.756 \% 000+00$
- $4987100+00$

-. 3.743401$)+00$

$.9904400+00$

-. $3937700+00$

$.9995700+00$

$-.99 .32 .900+00$

- $9970100+00$ $-.9904400+00$

- $304040 i+0 ?$ -. $97 E 2400+00$

$.9353900+00$ ..9559100+cn

- $9821 \% 00+: 0$ -. $92 ? ? 501)+(0$

$.9738500+00$ $.8967600+00$

- $5639.900+00$ -. $8585300+00$

- $9525600+00$

-.3148000+00

- $7396500+0$

$-.7653700+00$

- $9252100+00$ $-.7120300+00$

$.9043000+0)$ $-.6536+60+00$ 
HP23 : TRIGONDMETRIC APPROXIMATION PROBLEM

$101 \quad 11$

$.1000000+01$

- $1000000+01$

.0000000

$.1010050+01$

- $9998000+00$

- 4998000-01

- $1020200+01$

$.9992000+00$

$.9983000-01$

- $1030450+01$

. $3932000+00$

$.1494400+00$

. $1040810+01$

-.3963000+00

$.1986700+00$

- $1051270+01$

- $7950000+00$

$2+74000+00$

- $1061340+01$

- $9928100+u$

. $2955200+00$

. $1072510+01$

- $3902200+00$

- $3429 \mathrm{co0}+00$

- $1083290+01$

. $9972300+00$

. $3374200+00$

. $1094170+01$

- $9938400+00$

- $4349700+00$

- $1105170+01$

- $9800700+u J$

- $4794300+00$

- $1116280+01$

- $3759000+00$

- $5226900+00$

- $1127500+01$

- $\rightarrow 713400+00$

- $56464 \mathrm{CO}+00$

- $1138830+01$

- $9(: 53900+1) 3$

. $6051900+00$

$.1150270+01$

-.1610600+0J

$.6442200+00$

- $1161830+01$

. $9553400+00$

- $683164(0+0)$

- $1173510+01$

- $9492400+00$

$.71736,00+10$

- $1135300+01$

- $3427500+00$

- $7512300+00$

$.1147220+01$
- $1000000+01$ - 0000000

- $1000000+01$

- $1000000+01$

. 3000000-01

- $9987500+00$

- $1000000+01$

- 5996000-01

- $5950000+00$

- $1000000+01$

- $3582500-01$

- $92877 \mathrm{cutuj}$

- $1000000+01$

. $1197100+01$

- $9<00700+00$

- $1300000+01$

- $14944 \mathrm{~L} 0+0 \mathrm{~J}$

- $9089100+0 J$

- $10000011+01$

- $1790300+00$

. $9553400+00$

- $1000000+01$

- $2084000+00$

- $9393700+00$

- $1000000+01$

- $2377000+00$

. $9210600+00$

- $1000000+01$

- $2667300+00$

- $9004500+C u$

- $1000000+01$

- $2955200+00$

- $8775300+00$

- $1000000+01$

$.32404 i 0+00$

- $8325200+00$

- $1000000+01$

- $3522700+00$

- $8253400+00$

- $1000000+01$

$.33019010+00$

- $7900303+00$

- $1200000+01$

- $4077 i j 0+00$

$.7643400+00$

- $100000 u+01$

$.434 \% 700+00$

- $7316900+00$

- $1000000+01$

- $4617800+0 J$

- 6$) 671(1)+00$

- $1000000+01$

- $4381800+00$

. $6599800+00$

- $1000000+01$
.0000000

$.1000000+01$

- $1000000-01$

$.9995500+00$

- 2000000-01

- 3932000+00

- $30002150-01$

. $5957500+00$

- 3999000-01

. $43213100+00$

$.43481700-.1$

- $9487760+00$

. $5996000-01$

$.5833400+0 n$

- 6994000-01

$.9780 .300+00$

- $70910010-01$

$.9713400+00$

- $8988000-01$

$.9637700+00$

$.9933000-01$

$.9553400+00$

- $1097800+00$

- $9460400+00$

- $1197100+00$

- $9359000+00$

- $1296300+00$

$.9249100+00$

- $1395400+00$

- $91.30000+00$

- $1474400+00$

- $9004500+00$

- $1543200+00$

. $8369900+0$ i)

- $15 y 160 u+00$

. $8727400+00$

$.1790 .300+00$
- $1000000+111$

- 0000000

$.9959500+1) 0$

- 399700 0-01

- $4998000+00$

- $7 \div 010)(0-1) 1$

- $9995500+00$

$.1157100+(3$

- $9092000+00$

$.1593230+00$

- (9885500+ 2.)

- 1 ¿se $70(j+0)$

- $348=13) 100$

- $2377000+00$

- $9975500+i 0$

- $2763+03+00$

- $90653010+60$

- $31+5700+00$

- $75=540+00$

- उ5??700+00

- 9452000+00

$.3804200+00$

- $4939600+00$

-4?54400+un

- $9928100+00$

. $4617800+10$

$.9315600+00$

- 496 risiso+00

-990ะ200+00

. $5311 \mapsto 00+00$

$.9887700+00$

. $564(140)+00$

. $9372360+10$

. $5 \mathrm{c} 7 \mathrm{2}(0) 0+0)$

$.9855800+00$

$.6237900+00$

. $38338400+00$
- anciona

- $1000300+01$

- 200.$)(10)-(1$

$.9992000+00$

- 39c. $3001-c 1$

- acte a) $00+00$

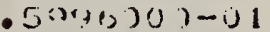

- 34.28100$)+(1)^{2}$

$.7091100 \mathrm{i}-\mathrm{Cl}$

- बह? ? 30$)+C: 1$

- :19300-31

- 43$) \cup ?(10+c)$

- $1177100+3)$

- $\left.712^{2}+31\right)+0: j$

. $1395401+c 4$

- $9010600+00$

- 1503201$)+013$

- $0492400+10$

$.1700 .300+(0)$

. 93590 onton

$.1986700+00$ $.3210600+00$

-2. $1.32300+00$

- 9u47500+00

. $2377000+c 0$

- $\{, 369,00+00$

- $2570300+00$ - Eं $78200+00$

- $27 E 3600+00$

- $3472007+0$ i

- $39552(10+0)$

- 8253400+00

$.3145700+00$ -2.21000+Co

$.3334900+00$

$.7775700+00$

$.3522 .770+00$ 
$.9359000+00$

$.7833300+00$

. $1209250+01$

$.9286600+00$

. $8134200+00$

$.1221400+01$

$.9210600+00$

. $\$ 414700+00$

- $1233630+01$

- $3130900+00$

- $3674200+00$

- $1246080+01$

$.9047500+00$

. $8912100+00$

- $1258600+01$

. $8960500+00$

- $9127000+00$

- $1271250+01$

- $9809900+00$

$.9320400+00$

- $1284030+01$

. $8775300+00$

. $9489800+00$

$.1296930+01$

. $8678200+03$

- $9635600+00$

$.1309960+01$

. $8577100+00$

- $97572 \mathrm{CO} 00$

. $1323130+01$

. $3472600+00$

$.9854500+00$

- $1336430+01$

- $3364000+c 0$

- $5927100+00$

$.1349360+01$

. $8253400+00$

$.9974900+00$

$.1363430+01$

. $8138800+00$

$.9937800+00$

- $1377130+01$

$.3021000+00$

. 1995700+00

$.1390970+01$

$.7899900+00$

- $99687 \mathrm{C0}+00$

$.1404550+01$

$.7775700+00$

. $4516600+00$

$.1419070+01$

. $7643400+00$

- ') $339900+00$

- $1433330+01$

. $7518100+00$

. $3738500+00$

. $1447730+01$

$.7384700+00$
- $5141400+00$

. $6216100+00$

$.1000000+01$

$.5396300+00$

. $5816800+00$

- $1000000+01$

$.5646400+0 u$

$.5403000+00$

$.1000000+01$

$.5891400+00$

$.4975700+00$

- $1000000+01$

$.6131200+00$

$.4536000+00$

. $1000000+01$

$.6365400+00$

$.40845 \mathrm{CO}+00$

- $10000010+01$

$.65938 \cup 0+00$

. $3623600+00$

$.1000000+01$

. CB16400+00

. $315326,0+00$

$.1000000+01$

$.7032800+00$

- ? $675000+00$

- $1000000+01$

. $7242900+00$

$.2190100+00$

$.1000000+01$

$.7446400+00$

- $1095700+00$

$.1000060+01$

$.7643300+00$

. $1205000+00$

. $1000000+01$

$.7833300+00$

$.7074000-01$

$.1000000+01$

$.8016200+00$

. 2079000-01

$.1000000+01$

. $8191900+00$

-.2920000-01

$.1000000+01$

- $8360300+00$

-.7912000-01

- $1000000+01$

- 8521110+00

-. $1288400+00$

- $1000000+01$

- $3674200+00$

- $1782500+00$

- $1000000+01$

- $8319000+00$

$-.2 ? 72000+00$

- $1300000+01$

. $83 \cdot 357000+00$
$.8577100+00$

- $1888000+00$

$.8419000+00$

$.1986700+00$

$.8253400+00$

- $2084600+00$

$.8030300+00$

$.2182300+00$

. $7890900+00$

$.2279300+00$

. $7712500+00$

$.2377000+00$

$.7516100+00$

$.2474000+00$

$.7316900+00$

- $2570800+00$

$.7100100+00$

$.2667300+00$

$.6895000+00$

$.2763600+00$

$.6674600+00$

$.2859500+00$

$.6448304+00$

$.295520 n+00$

$.6216100+00$

$.3050600+00$

$.597830 u+00$

$.3145700+00$

$.5735200+00$

$.3240400+00$

$.5486900+00$

. $33334900+00$

$.5233700+00$

$.3429000+00$

$.4575700+00$

. $352.2700+00$

$.4713300+00$

$.3616200+00$

$.4446,000+00$
$.6593300+013$

$.751: 3100+n o$

$.9820000+00$

$. .3704200+00$

$.6889200+00$

$.73 .484100+0.3$

$.0800700+00$

$.7173000+00$

- $38994 ? 00+00$

of $96: 710 u+0)$

- $9780300+0 n$

$.7440400+n$.

$.9753000+00$

$.770 ? 400+60$

$.9736700+00$

- 795600000

$.971340 J+0 c$

- $81 \div 1000+00$

$.9639100+00$

$.3414700+00$

- $96(3) 300+00$

- $3624000+00$

$.46,37700+00$

. $3319600+00$

- $9610600+00$

$.9001000+00$

. $9582400+00$

- $9164000+00$

$.95534110+00$

$.9320400+00$

$.952 .1500+00$

. $457800+n n$

$.9492400+00$

- 958narotio

$.9460400+00$

- $9687210+00$

$.94: 7500+00$

$.9778600+00$

$.9393700+00$

$.9854500+20$

$.9356000+00$

- csc $14600+100$

$.9323300+00$

. $0458300+00$
$.4077000+00$

$.6074501+00$

- $42 ! 30407+01)$

- $6571500+10$

$.44 .3 \% 500+0 \%$ $.60,2,200+($.

$.46,174(3)+(0)$ . $\{7353: 21 \%+(0$

$.4794300+1$ . $340: 3000+v 0$

- 4:- $8800+00$ - $5002 ? 31+0$ ?

$.5141403+00$ $.4713300+00$

$.53110000+00$ - $<3$ stessil

$.5450(0): 10+(10)$

- 32) $30(10+0)$

$.5546,400+00$ . $3023600+1)$

$.5310400+00$ . $3248020+00$

$.913720011+010$ - $28+72010+00$

- E.131?(0+0) $.248180 u+0 r$

- E.2370nntid . $2092400+00$

$.6442211+00$ $.1649700+60$

$.0,547801)+00$ $.130420 n+00$

- $6742 \div 30+00$ . $\leftrightarrow 0670 \mathrm{CO} 0-01$ 
- $4612800+00$ - $1462280+01$ - $7248400+00$ . $9463000+00$ - $1476980+01$ $.7109100+00$ - $9289600+00$ $.1491820+01$ . $6967100+00$ . $9093000+00$ - $1506820+01$ . $6822200+00$ - $38736 \mathrm{CO}+00$ . $1521960+01$ .6674600+00 - $\$ 632100+00$ - $1537260+01$ $.6524400+00$ - $8369000+00$ $.1552710+01$ $.6371500+00$ $.8085000+00$ $.1568310+01$ $.0216100+00$ $.7780700+00$ $.1584070+01$ $.60582 .00+00$ $.7457100+00$ . $1599990+01$ . $5397900+00$ . $7114700+00$ $.1616070+01$ . $5735200+00$ - $6754600+00$ - $1632320+01$ $.5570200+00$ . $6377600+00$ - $1648720+01$ $.54 J 3000+00$ - $5384700+00$ - $1649720+01$ . $5233700+00$ . $5576800+00$ $.1648720+01$ . $5062200+00$ - $5155000+00$ $.1643720+01$ - $48387 \mathrm{CO}+00$ $.4720 .300+00$ $.1648720+01$ . $4713300+00$ - $4273.310+0 n$ - $1643720+01$ . $45.36000+00$ . $3816600+00$ $.1643720+41$ . $43568 \mathrm{CO} 0+0$ $.3349900+i j$
$-.2755900+00$ - $1000000+01$ - $9086300+00$

- $3232900+00$ - $1000000+01$ . $9207500+00$

-.3701300+00 - $1000000+01$ - $9320400+00$

-.4161500+00 - $1000000+01$ - $9424900+00$

$-.4610700+00$

- $1000000+01$ $.95209 \mathrm{CO}+00$

$-.5048500+00$ . $1000000+02$ - $9608400+00$

-. $5473600+00$ . $1000000+01$ . $9687200+00$

$-.5885000+00$ - $100000 J+01$ . $9757200+00$

$-.6281700+00$ - $1000000+01$ - $9818500+00$

$-.6662800+0$ ? - $1000000+01$ - $9871000+00$

-. $7027100+00$ - $1000000+01$ - $9914600+00$

- .73739 $\mathrm{CO}+\mathrm{CO}$ - $1000000+01$ . $3749200+00$

$-.7702300+00$ - $1000000+01$ - $9974900+00$

-.8011400+00 - $1000000+01$ - $3991700+00$

$-.8300500+00$ - $1000000+01$ - $9999400+00$

-.8568900+00 - $1000000+01$ - $9998200+00$

- $88158 \mathrm{co}+00$ - $1200000+01$ - \$987500+00

-.904J700+0j $.1000000+01$ - $9963700+00$

$-.924 .3000+0 J$ - $1000000+01$ - $9943400+00$ -. $9422200+00$
. $3709200+00$ - $4175900+00$

- $3801900+00$ . $390.1500+00$

- $3894200+00$ $.3623600+00$

$.3986100+00$ $.3342400+00$

$.4077600+00$ $.305832 .00+00$

$.416,3700+30$ . $2771200+00$

. $4259400+00$ . $2431800+00$

$.4349700+00$ . $2190100+00$

$.44 .39500+00$ - $1896400+00$

- $4528900+00$ - $1601000+00$

$.4617800+00$ $.1304200+00$

$.4706300+00$ - $1006300+00$

- $4794300+00$ $.7074000-01$

- $4881820+00$ . $40790 \cup 0-01$

- $4968800+00$ - $1080000-01$

$.5055300+00$ -.1920000-01

$.5141400+00$ $-.4918000-01$

$.5226900+00$ -.7912000-01

$.5311900+00$ $-.1089900+00$
$.9286000+00$ - $9987100+00$

- $9249100+00$ - $959 \% 400+00$

- $9210600+00$ - $9965700+00$

- $9171200+00$ - 9976100+00

- $9130900+00$ $.9940400+00$

$.9089700+00$

- $9330700+C 0$

- $0047500+00$

$.9821500+00$

$.900+500+00$

$.9738510+00$

- $8960500+00$ $.9639800+0.0$

- $8915700+00$ $.9525800+00$

$.8369900+00$ $.9396500+00$

$.8823300+00$ - $92.521 \mathrm{CO}+\mathrm{CO}$

$.8775800+00$ $.9093000+0)$

$.8727400+00$ - $8919300+00$

$.8673200+100$ $.8731300+00$

- 36 2es 1110+co - 8529401$)+00$

- $\$ 57710.0+00$ . $8313800+00$

- $85.35200+00$ $.8085000+02$

$.8472600+00$ $.7843200+00$
- $6889200+00$ - $5077000-n 1$

$.7032300+00$ -1090:00-u1

- $7173000+0 \%$ $-.2920000-11$

$.731150 n+03$ $-.69150) 0-01$

$.7446: 400+13$ $-103 x \cos 00$

- 75783401$)+00$

-. $14365 n o+$ r

$.7707400+(2)$

$-.1880800+(1)$

$.7+3.3310 \hat{3}+00$ $-.2272000+00$

- $7956000+00$ $-.265960^{\circ} 0+00$

$.8075600+00$ $-.3043000+00$

$.8191900+00$ $-.34 ? 1500+10$

- $8305000+00$

$-.379450 \mathrm{c}+\mathrm{c})$

$.8414700+00$

$-.41615\left(10+0^{\prime}\right)$

- $9521110+00$ $-.4521 \because 10+00$

- $83624.000+00$ $-.4874300+00$

- $3723600+00$ $-.5220110+0$

- $8313: 30+00$ $-.5557000+0 \mathrm{C}$

$.8912100+00$ $-.5885000+c 0$

- $9001000+100$ - $6.2030 .00+00$ 
- $1648720+01$

$.4175900+00$

. $2874800+00$

. $1648720+01$

. $3993400+00$

$.2392500+00$

. $1648720+01$

- $3309200+00$

. $1904200+00$

. $1648720+01$

- उE $23600+00$

$.1411200+00$

$.1648720+01$

$.3436500+00$

$.9146000-01$

$.1648720+01$

$.3248000+00$

$.4158000-01$

. $1648720+01$

. $3058200+00$

-.8410000-02

$.1648720+01$

- $2867200+00$

-.5837000-01

$.1648720+01$

. $2675000+00$

-. $1082000+00$

$.1648720+01$

$.2481800+00$

- .1577500+00

$.1648720+01$

. $2287500+00$

$-.2069000+c 0$

. $1648720+01$

$.2092400+00$

$-.2555400+100$

- $1 \epsilon 48720+01$

- $1890400+00$

-. $3035400+00$

$.1648720+01$

$.1699700+00$

-. $3507800+00$

$.1648720+01$

$.1502300+00$

-.3971500+00

$.1648720+01$

$.1304200+C 0$

$-.4425200+00$

$.1648720+01$

$.1105700+00$

$-.4867900+00$

$.1648720+01$

$.9067000-01$

-. $5298400+00$

$.1648720+01$

$.7074000-1) 1$

$-.5715600+00$ $.1648720+01$
$.1000000+01$

. $9903300+00$

-.9577500+00

- $1000000+01$

$.9857200+00$

$-.9709600+00$

$.1000000+01$

$.9802200+00$

$-.9817000+00$

$.1000000+01$

$.9738500+00$

$-.9899900+00$

$.1000000+31$

$.9665900+00$

$-.9958100+00$

$.1000000+01$

. $9584700+00$

$-.9991400+00$

. $(000001)+11$

$.9494900+00$

-. $5999600+0$ )

. $1000000+01$

- $9396500+00$

-. $5982900+00$

$.1000000+01$

-3289600+00

-.9941300+00

. $1000000+01$

$.9174400+00$

$-.987480 u+00$

- $1000000+01$

. $9050900+00$

$-.9783600+00$

$.1000000+01$

$.8919300+00$

$-.9668000+0$ ?

$.1000000+01$

. $8773600+00$

-.9528200+00

- $1000000+01$

$.8632100+00$

- $\$ 364600+00$

- $1000000+01$

. $8476800+00$

$-.9177500+00$

- $1000000+01$

$.8313800+0()$

-.8967600+00

$.1000000+01$

$.8143400+00$

$-.8735200+00$

$.1000000+01$

$.7965700+00$

$-.8481000+00$

$.1000000+01$

$.7780700+00$

$-.8205600+00$

$.1000000+01$
$.5396300+00$

-. $1387500+00$

$.5480200+00$

-. $1684000+00$

$.556 .3600+00$

-. $1978900+00$

$.5646400+00$

$-.2272000+00$

$.5728700+00$

$-.2563100+00$

$.5810400+00$

$-.2851900+00$

$.5891400+00$

-.3138100+00

$.5972000+00$

$-.3421500+00$

$.6051900+00$

$-.3701800+00$

$.6131200+00$

-. $3978800+00$

$.6209900+00$

$-.4252200+00$

$.6287900+00$

$-.4521800+00$

$.6365400+00$

$-.4787300+00$

$.6442200+00$

$-.5048500+00$

$.6518300+00$

$-.5305100+00$

$.6533800+00$

$-.5557000+00$

$.6068700+00$

$-.5803900+00$

$.6742900+00$

$-.6045500+00$

. $6816400+00$

-.62B1700+00

$.6839200+00$
$.6415000+00$

$.7588800+00$

- $9086.300+00$

$-.6512300+\mathrm{co}$

- $8364600+0)$

$.732 .2300+c 0$

$.8309400+00$

$.7044100+0$ )

$.8253400+00$

- $6754600+00$

. $31965 \cdot 20+0 !$

. $6454300+00$

$.8138800+00$

$.6143700+00$

$.8080300+00$

$.5823300+0 u$

$.8021000+00$

$.5493600+00$

$.7960800+00$

$.5155000+00$

$.7399900+00$

$.4808200+00$

. $7838200+00$

$.4453700+00$

$.7775700+00$

$.4092100+00$

$.7712500+00$

$.3724000+00$

- $76,48400+00$

- $3349900+00$

$.7583600+00$

$.2970400+00$

$.7518100+00$

. $2586200+00$

$.7451700+00$

$.2197800+00$

$.7384700+00$

- $1806000+00$

$.7316700+30$

$.1411200+00$

$.72453400+00$
- $7169020+70$

. $6310 \% 100+00$

- $92461(1) 0+c 0$

$-.70979) 0+0 ?$

- $9320+00+00$

$-.7373300+00$

$.9391300+01$

$-.7634200+00$

. 5457 300+00

$-.7890100+00$

-452.0000+00

-.8129500+0)

- $95 i j 0 \geq 00+00$

- $8.355000+31$

$.9635600+03$

$-.8563300+00$

$.9687200+00$ -.876 \$3.00+00

. $9734300+00$ $-.895 .340 \mathrm{C}+\mathrm{CO}$

- $9778600+00$

$-.0124400+10$

$.9818500+00$

$-.9280700+00$

- $9854500+00$

$-.94 ? 2200+00$

-. $3886500+00$

$-.9548600+110$

$.99146010+00$

$-.9650800+00$

. $79.39700+00$

$-.9755500+0 u$

- $995830 \mathrm{c}+00$

$-.9835500+00$

$.9974900+00$

$-.980990)+00$

. $9987100+00$ 
$.5077000-11$

- 6118601$)+1,0$

- $1648720+1,1$

$.3079000-01$

-. $5506300+.10$

. $1648720+01$

. $1080000-01$

-.6877700+00

. $1648720+0.1$

$-.9200000-02$

$-.7231900+30$

$.1648720+01$

-.?920000-01

-.7568C60+(1) - $1648720+01$

-.4918000-i1

-.7885.300+10 $.1648720+J 1$

-.0715000-U1

-.8182800+00

- $1643720+(11$

$-.890 \geqslant 000-61$

-. $945930 \mathrm{C}+00$

- $16: 48720+1) 1$

-. $1039900+00$

-.3715800+00

- $16487 ? 0+v 1$

- $1229400+00$

- .3y44900+uo

- $1648720+01$

- $1486500+00$

-. $16.1700+00$

. $1648720+v 1$

-.1684000+00

-. .350500+00 $.1648720+01$

$-.188080 .3+1.0$

$-.95160 \mathrm{cot}+30$ - $1648720+(11$

-. $2076800+00$

-. $3 \epsilon 5770 \mathrm{~J}+\mathrm{C}$ - $1648720+01$

-.2272000+00

-.3775300+10 - $1648720+01$

- $24(.6 .300+00$

-. $9868400+1.0$ - $1648720+11$

- 2559000+(10

- $9936900+00$ - $16483720+01$

- $2851900+110$

-.9980500+10

- $1048720+c 1$

-.30436.00+6.0

-.5959200+10

- $16487 z 0+i 1$

$-.3232400+1.0$
- $7588800+00$

$-.7909700+0.1$

- $1000000+02$

$.7390100+00$

$-.7594000+00$

- $1000000+01$

$.7184600+00$

-. $7259300+00$

. $1000000+01$

- $69728 \mathrm{cot} 00$

-.6906500+00 - $1030000+01$ - $6754600+00$

- $6536400+00$ - $1000000+01$ - $5530400+00$

- $6150000+00$ - $1000000+01$ - $6300300+00$

-.5748200+00 - $1000000+01$ $.0064500+00$

$-.5332100+00$ - $1000000+01$ - $5823300+00$

- $450 ? 600+00$ - $1000012+01$ . $5576800+00$

-.440.0900+00 - $1000000+01$ . $5325300+00$

-.4008000+00 - $1000000+01$

- $506 \div 1 \mathrm{co}+00$

-.3545100+00 $.1000000+01$

- $48082 \mathrm{co}+00$

-. $3073300+00$ - $1000000+01$ $.4543100+00$

-. $2593900+00$ - $1000000+01$ - $4273800+00$ $-.2108000+00$ - $1000000+01$ - $40007 \mathrm{CO}+00$

- $1616800+00$

- $1000000+01$ . $3724000+00$

-.1121500+00

- $1000000+01$

- $3443900+00$

-. $f: 22500.0-01$

- 1.j) ט $0+v 1$

- $3160800+00$

-.12.39000-01

- $1000000+01$

. $2474800+00$
$-.6512320+00$

$.1014200+00$

$-.9948400+00$

$.5901400+00$

$-.6737000+00$

$.70 .32 .800+00$

$-.6955600+00$

$.7103500+00$

$-.7158000+00$

- $7173600+00$

$-.7373900+00$

- $7242900+00$

-.75?3200+00

. $7311500+00$

-. $7765700+00$

$.7379300+00$

$-.7951200+00$

$.7446400+00$

-.8129500+00

$.7512800+00$

$-.3300500+00$

$.7578400+00$

-.8464100+00

$.76433300+00$

- 86? oriootoo

$.7707400+00$

$-.3700200+100$

$.7770700+00$

-.8908500+0:

- $7833300+00$

$-.3040700+00$

- $7895000+00$

$-.916,4800+00$

$.7956,000+00$

$-.9240700+00$

- $5016200+00$

$-.9388300+00$

- cutis.0u+vo

-.9487300+00

$.9134200+00$

$-.9577900+00$ $.7173100+\cdots 0$ $.61550(10-1 ; 1$

$.710 \geqslant 100+(10$

$.2154000-01$

$.7038500+00$

$-.1541000-01$

- $6967100+00$

$-.5837000-01$

- $6595000+00$

$-.9825000-01$

- $64272 \mathrm{CO}+00$

$-.1379700+00$

$.6748800+c 0$

$-.1774600+00$

-c. $74600+00$

-.2166.800+00

- $(i \leq 9) 3(1)+(1 n$

$-.2555400+00$

- $6524400+00$

- . $2: 40000+00$

$.6448300+00$

-.3319900+00

- $6.371500+00$

$-.3694400+00$

$.6294100+00$

-.40(i.110i)+i)0

$.6216 .100+00$

$-.4425 ? .00+00$

.6137500+00

$-.4780300+00$

- $6(15,23) 0+00$

$-.5127700+00$

- $5 \div 75.300+00$

$-.5466: 300+00$

- 5r: $8,00 j+6$,

$-.5737400+0 u$

- 581680$)+00$

-.6112600+0
- $799530 \%+00$

- $4981600+00$

- $9593400+00$

-. $9957 \% 00+00$

$.999: 360+00$

-. $900 \div 200+00$

- $3405700+00$

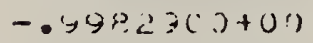

- 99 9.500)+00

-.4651600+00

-.976100+00

-.x904402+00

- $996.0200+00$

-.9841300+00

- $5940400+00$

$-.176 .2+00+00$

$.991+6,00+00$

-. $966 r 3000+00$

- $988: 3700+00$

$-.055 \times 100+00$

- $9857: 200+00$

$-.9432300+00$

- $982.1 \% 010+00$

$-.9292 ; 00+00$

$.9762000+00$

$-.91371+00+00$

- $9733 ; 00+00$

- 89 tr $76001+00$

- Stíliooton

-. $378.3 \leq 0 j+00$

- $4615.13 u+00$

- . $85+5300+00$

$.45+? 01)+03$

-.8377:300+00

- $625: 01+00$

$-.814 \cdots 1100+00$

$.9463 .100+0.7$

$-.7306 .700+00$ 


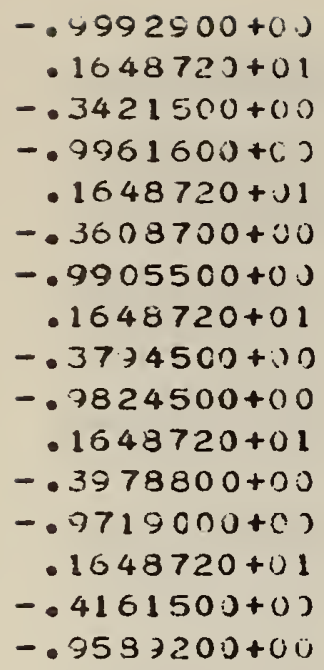

-.9533200+0u

- 376.0000-01 $.1000000+101$

- $2586200+00$ - 8751)000-01

$.1000300+01$

. $2295300+00$

- $1371300+00$

- $1000000+01$

. $2002300+00$

- $1865100+00$

$.1000000+01$

- $1707500+00$

- $2353800+0 J$

- $1000000+01$

$.1411200+00$ $.2836600+00$
- $3101900+00$

- $5659800+00$

- $8248900+00$

-. $5733000+00$

- $8305000+0 \mathrm{C}$

$-.9797500+00$

- 836.0300+00

- $9853100+00$

- $8414700+00$

-. $39990+00$
$.5735000+70$ - $6 \cdot+30000+00$

$.5653000+6=$ $-.6731100+00$

$.5570200+00$ -.7021500+00

$.5435 .460+00$

$-.730(1000+1) 0$

$.5403000+00$

$-.7565000+00$

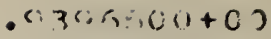

-.7仑5870u+v0

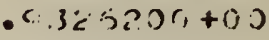
-.7355. $00+00$

$.9252100+00$ $-.7120300+C 0$

- $7174+00+n 0$ - $4: 38300+00$

$.505 \operatorname{sn} x+00$ $-.6536402+00$ 
HP24: TRIGCNEVETFIC APPICXIMATICN PREHIE

$201 \quad 11$

$-1000000+01$

$.1000000+01$

- 3000000

- $1005010+01$

. $9999500+00$

. 2500000-01

- $1010050+01$

- $9998000+00$

.4998000-01

- $1015110+01$

- $3995500+00$

$.7493000-C 1$

- $1020200+01$

- $9992000+100$

- 9983000-01

$.1025320+01$

- $9987500+00$

- $1246700+00$

- $1030450+01$

- $9982000+00$

- $1494400+00$

- $1035620+01$

- $9975500+00$

- $1741100+00$

- $1040810+01$

- $9968000+00$

- $1986700+00$

- $1046030+01$

- $9959500+00$

- $2231100+00$

- 1 C $51270+01$

- $9950000+00$

. $2474000+00$

- $1056540+01$

- $9939600+00$

. $2715500+00$

- $1061340+01$

- $3928100+00$

- $2955200+00$

- $1067160+01$

- $9915600+00$

- $3193100+00$

- $1072510+01$

- $3902200+00$

- $3429000+00$

$.1077880+01$

- $9537700+00$

- $3662700+00$

- $1083290+01$

- $9872300+10$

. $3894200+00$

- $1088720+01$

- $9855300+00$

- $4123200+00$

$.1094170+01$
- $1000000+01$

. 0000000

- $1000000+01$

- $1000000+01$

- $1500000-01$

- $\$ 996500+00$

- $1000000+01$

- 3000000-01

- $9987500+00$

- $1000000+01$

- 44:78000-01

- $5971900+00$

- $1000000+01$

- 59y12000-01

- $9950000+00$

- $1000000+01$

$.749 .3000-01$

- $9922000+00$

- $1000000+01$

- 358800J-01

- $9387700+00$

- $1000000+01$

- $1048100+00$

- $9847300+00$

- $1000000+01$

- $1197100+00$

- $9800700+00$

- $1000000+01$

- $1345900+00$

- $97475 \mathrm{CO}+00$

- $1000000+01$

- $1494400+00$

- $5689100+00$

. $1000000+01$

$.1642500+00$

- $9624300+00$

- $1000000+01$

- $1790300+00$

$.9553400+00$

- $1000000+01$

- $1337700+\mathrm{CO}$

- $9476500+00$

- $1000000+01$

- 2 गत4 $600+00$

- $9393700+00$

- $1000000+01$

- $2231100+00$

- $9305100+00$

- $1000000+01$

- $2377000+00$

- $9210600+00$

- $1000000+01$

- $25225 \mathrm{co}+00$

- $9110400+00$

- $1000000+01$
- 0000000

$.1000000+01$

- 5c00000-02

. $9939900+00$

- 1000 100-01

. $9995500+60$

- $15 \cdot 3 \cap 000-01$

- $4,3+900+00$

- $2300000-01$

- S5: $2000+60$

- $2500000-01$

. $9971900+00$

- 3000000-01

.5954500+00

- 34(39000-01

- $9944700+00$

- 3939000-01

. $9928100+00$

-4498000-01

- $9900000+00$

- 49980 00-01

- $9887700+00$

$.5477000-01$

. $9864200+00$

- 5996000-01

. $9838400+00$

. $6495000-01$

. $9810500+00$

.6994000-01

$.9780300+00$

- 7493003-01

. $9747900+00$

- 79231000-01

$.971 .3400+00$

- 3490000-01

$.9675600+00$

$.8988000-01$
- $1000000+01$

.0000000

- $995 \leq 900+c 0$

.2000000-01

- $9959500+00$

- 3c $50000-01$

- $95,53900+00$

- 5498,OC a-01

- $9575000+00$

- 75 $91000-01$

- 9งc5บ0)+0.?

- $9783000-01$

- 9555500+00

$.11 \zeta 7100+00$

- $9993900+(0)$

$.1305400+30$

- $9952000+00$

- $1593200+00$

- $9989900+01)$

. $1790300+00$

$.9987500+00$

$.1986700+00$

- $9984900+00$

. $2192300+00$

- $9982030+00$

$.2377000+00$

- $9978900+01$

$.2570300+01)$

- $5075500+i)(1$

. $27636,20+00$

- 9971 co(:0) 00

$.2455230+x$

- $\operatorname{sic} 3000+00$

$.3145700+00$

- $9903900+00$

. $3334900+00$

$.995551) 0+00$
- 01) untio

- $1000000+01$

- $1000000-01$

- y95000+00

-?(10.):000-01

-scos.s0u+0o

- $300(0(0)-i) 1$

- $998 ? 300+90$

- 3950903 01

- $\$ 963() 0+$.

- 4 4. $\div 200-01$

. $9950300+C 0$

-5996000-01

- $9928100+00$

-6994000-01

- $9902200+00$

- 7991000-01

- 987 ? $300+00$

- $89834003-01$

- $9838410+00$

- $9983: 300-01$

- $9800700+00$

- $1077800+00$

. $9759000+00$

$.1197100+00$

$.3713400+00$

- $1296300+00$

$.9663700+00$

- $1335400+00$

- $46,10600+30$

- $1494400+00$

- 955340$)+00$

- $156,3 \geq 00+00$

- $r 40240 n+00$

$.1691800+00$ . $9427500+00$

$.1790300+00$ 
$.9838400+00$ $.4349700+00$ . $1099660+01$ . $9823000+00$ $.4573400+00$ . $1105170+01$ - $7800700+00$ . $4794300+00$ $.1110710+01$ $.4780300+00$ $.5012100+00$ $.1116280+01$ - $9759000+00$ . $52269 \mathrm{CO}+00$ $.1121870+01$ $.9736700+00$ $.5438300+00$ - $1127500+01$ $.9713400+00$ $.5646400+00$ $.1133150+01$ $.9689100+00$ - $5851000+00$ $.1138830+01$ $.9663900+00$ . $6051900+00$ $.1144540+01$ - $9637700+00$ $.6243000+00$ . $1150270+01$ $.9610600+00$ $.0442200+00$ - $1156040+01$ $.9582400+00$ $.6631400+00$ $.1161830+01$ $.9553400+00$ $.68164 \mathrm{CO}+00$ - $1167 \epsilon 60+01$ $.9523300+00$ $.6997200+00$ . $1173510+01$ - $9492400+00$ . $71736,00+00$ . $1179390+01$ $.9460400+00$ - $7345500+00$ . $1185300+01$ $.2427500+00$ . $7512300+v 0$ - $1191250+01$ $.9333700+00$ $.7075400+00$ - $1197220+01$ $.9359000+00$ $.7833300+00$ $.1203220+01$ . $9323300+00$
- $26,67300+00$ . $9004500+00$ $.1000000+01$ $.2811600+00$ . $8892900+00$ - $1000000+01$ - $2555200+00$ . $8775800+00$ - $1000000+01$ $.3098200+00$ $.8653200+00$ - $1000000+01$ $.3240400+00$ $.8525200+00$ $.1000000+01$ - $3382000+00$ $.3391900+c 0$ - $1000000+01$ . 35<2 $2700+00$ $.8253400+00$ $.1000000+01$ - $3 t 62700+00$ . $8109600+00$ $.1000000+01$ $.3801900+00$ - $7500800+00$ $.1000000+01$ $.3940200+00$ - $7807100+00$ . $1000000+01$ $.4077600+00$ . $7648400+00$ - $1000000+01$ - $4214100+00$ $.7485000+00$ $.1000000+01$ $.4349700+00$ $.7316900+00$ $.1000000+01$ $.4484200+00$

. $7144200+00$ - $1000000+01$ $.4617800+00$ $.6967100+00$ $.1000000+01$ $.4750300+00$ . $0785600+00$ . $1000000+01$ $.4881800+00$ . $5539800+00$ - $1000000+01$ $.5012100+00$ $.6410000+00$ - $1000000+01$ - $5141400+00$ - t. $216100+00$ . $1000000+01$ $.5269400+00$
$.9637700+00$

$.352 ? 700+00$

$.335 \div 000+00$

. 9486001$)-01$

- $5954000+00$

- $506600+00$

$.37032(0+) 0$

. $9983000-01$

- $\$ 553400+00$

- 50500notou

. $3344230+00$

$.1048100+00$

. $9508000+00$

$.9944900+00$

$.4077600+00$

- $1097800+00$

. $\$ 450400+00$

$.1147500+00$

. $\$ 410800+00$

- $1157100+00$

- $9.359000+00$

- $1246700+00$

- $3305100+00$

- $1296300+00$

$.9249100+00$

$.1345900+00$ $.9191000+00$

- $1395400+00$ $.9130900+00$

$.1444500+00$ $.9068700+00$

- $1494400+00$ - $9004500+00$

$.15438300+00$ $.89 .38200+00$

- $1593200+\mathrm{C} 0$ - $8860300+00$

$.1642500+00$ $.8799700+00$

$.1691800+00$ $.8727400+00$

$.1741100+00$ $.8653200+00$

$.1790300+00$ $.8577100+00$

- $1839500+00$ $.8459000+00$
. $3033000+: \cdot$

$.425 \subseteq 400+00$

- $9933900+00$

$.4439500+00$

$.9920100+00$

$.4617300+0$ )

. $9922000+00$

$.4794300+00$

$.9915600+10$

$.4963800+00$

- 99 $04000+00$

$.5141400+0$.

$.9002200+00$

$.5311500+00$

$.9895100+00$

$.5483200+00$

$.9887700+01$ $.5646400+00$

$.9880100+00$ $.5810400+00$

$.9872300+00$ $.59720(0+00$

$.9364200+00$ -6 $131200+00$

- $985558(0)+00$ - $62,7(3 i)+100$

$.9947300+00$ . 644?:30 00

$.98383400+00$ $.6593800+00$

$.9829400+00$ $.6742300+0$ )
- 1 E. ร. E.1,000+uo - osasiojotoo

- 1985700+ 30 $.9210<00+\angle 0$

$.2034<00+00$ - $9130900+00$

. $215.23(i n+0)$ $.50475\left(n^{\prime}\right)+00$

. $.227(481) ?+100$ - $336050 n+60$

. . $3779006+v 0$ - $(86) 3$ inoton

$.3474160+00$ $.5775: 00+00$

$.25703(10+0) 0$ $.8673300+00$

$.2667300+00$ .85771 . $0+00$

- $276.3600+c 0$ - E.47?sUD+co

. $2859500+00$ - $836,4600+110$

- 2955?00+00 $.8253400+00$

$.3050500+00$ . $81383.30+00$

- 314570()$+00$ . $8.21030+0$ ?

- 324.24110+0? - $734 \cdot 300+n$ n

$.2334000+100$ $.7775700+00$

$.3429000+00$ - TE 4 E Al: O +COO

- $3522700+00$ $.7519100+00$

- se $16000+00$ $.7 \times 84700+00$ 
- $7986200+00$ - $1209250+01$ - $928660 n+00$ . $8134200+00$ $.1215310+01$ . $9249100+00$ - $8277000+00$ - $1221400+01$ - $9210600+00$ - $8414700+00$ - $1227530+01$ . $9171200+00$ - $8547100+00$ - $1233680+01$ - $913 \mathrm{C} 900+10$ - $96742.30+20$ - $1239800+01$ . $9089700+u$ - $3795900+00$ - $1246080+01$ - $9047500+00$ . $8912100+00$ - $1252320+01$ - $9004500+00$ $.9022700+07$ - $1253500+01$ - $8960500+00$ - $9127600+00$ - $1204+10+01$ . $8915700+00$ . $92209 \mathrm{CO}+00$ . $1271250+01$ - $3869900+0 J$ . $9320400+00$ - $1277620+01$ - $8823300+00$ - $74081 \mathrm{CO}+00$ - $1284030+01$ . $8775800+00$ - $9485800+00$ - $1290460+01$ - $3727400+00$ . $9565700+00$ - $1296930+01$ . $3678200+00$ . $9635600+00$ - $1303430+01$ . $3628100+00$ - $9699400+00$ . $1309960+01$ - $3577100+00$ $.97572(0+0)$ - $1315530+01$ - $-5525200+00$ - $98089 \mathrm{cot} 00$ - $1.323130+01$ . $3472000+03$ . $9854500+00$
- $6018300+00$ - $1000 \mathrm{cou}+01$ - $5396300+00$

- $58168 \mathrm{CO}+00$

- $1000000+01$

- $5522000+00$

- 56,11700+00

- $1000000+01$

- $5646400+00$

$.5403000+00$

. $1000000+01$

- $5769600+00$

$.5191000+00$

- $1000000+01$

- $58914 \mathrm{Cu}+0 \mathrm{u}$

. $4975700+00$

- $1000000+01$

-6012000+00

- $4757300+00$

- $1000000+01$

$.61 .31200+00$

- $45300 \mathrm{co}+00$

- $1000000+01$

. $6247000+00$

- $4311600+00$

- $1000000+01$

. $6365400+00$

- $4084900+00$

- $1000000+01$

- $6480300+00$

- $3855400+00$

- $1000000+01$

. $6593800+00$

- $3623600+00$

- 100000$)+01$

. $6705900+00$

$.3389500+00$

- $1000000+01$

. $6816400+00$

$.3153200+00$

- $1000000+01$

.67254n0+00

- $2915 \mathrm{COO}+00$

- $1000000+01$

. $7032 \mathrm{CO}+00$

-2.675c00+00

- $1000000+01$

- $7138600+00$

- $24333 \mathrm{cj}+00$

- $10 \mathrm{0} 0000+01$

- $7 \therefore 42500+0 u$

- $2190100+00$

- 1000 C.joto 1

$.7345500+00$

- $1945500+00$

. $1030000+01$

. $7446400+00$

- $1659700+00$
- 1 e88cuvou

$.8719000+00$

- $1937700+00$

. $8337100+00$

- $1986700+00$

. $8253400+0$.

$.2035700+00$

$.8157700+00$

$.2084600+00$

. $3030300+00$

- $2133500+00$

- $7991000+00$

- $218230 n+00$

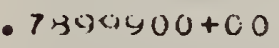

. $22.31100+00$

$.7807100+00$

$.2279800+00$

$.7712500+C J$

$.2328400+00$

. $7616100+00$

$.2377000+00$

$.7518100+00$

- $2425630+00$

- $7413300+00$

$.2474000+00$

$.7316300+00$

. $2522500+00$

$.7213800+00$

- $2570800+00$

$.7103100+00$

$.261 ? 100+00$

$.7002800+00$

- $2607300+00$

. $6895000+00$

. $27151500+00$

. $678500 u+00$

$.2763600+00$ $.667+600+00$
- 9320000+00 $.6887200+00$

- $81(1500+00$

- $7032800+C 0$

- $9800700+00$ $.7173600+00$

- $9790600+00$ $.7311500+?$

- $97803 \mathrm{vc}+0$ ? $.7446400+50$

- $9760800+00$ $.7575400+0 u$

$.975 \div 000+0.1$

$.77074 \mathrm{cu} 0 \mathrm{~J}$

$.9747 .000+(10)$

. 7 33.3.3.300+00

- $9736: 700+00$

$.7956000+0 u$

- $9725100+01$ . $8075600+0$

$.9713400+01$ . $8191900+0$ J

- 9701 it $00+011$ . $8305000+0$ )

- $953 \geqslant 100+00$ . $8414700+00$

- $9570600+01$ $.85 ? 1107+30$

- $9663900+00$ - 86:20.)0+u

- $6650300+00$ - $8723600+00$

- $96.37100+04$ - $831 ; 0 u+10$

$.9(. .74 .3 \cdot: 0+3)$ - $8 \geqslant 1 \div 100+00$

- $9510000+00$ - $9001000+0$ ?
- $7709200+30$

- $724 \varepsilon 400+00$

- 38.01300+00

$.7109100+00$

. $3874200+00$ $.6967100+00$

- $3986100+00$ - $(92222)+1,2$

- $4077(1) 0+(10$ - 674.1$)(1+C 0$

$.4158700+00$ - $05244: 10+i)$

- $425(1400+00$ - $6=71500+00$

$.4349700+00$

.6216102+00

$.44395(0+0)$

. $6058<00+i n$

. 45.281700+00 $.5897900+00$

. $4617800+00$ - $5735: 20 ?+00$

$.470 \in 3.70+00$ . $5570 ? 00+00$

$.4794300+00$ $.540 .3000+00$

- 4 \& 31 HOO +0 - $52337 \%)+0 !$

- $496383.20+00$ $.506: 230+00$

$.5055300+00$ . $4383700+07$

$.5141410+00$

$.471 .3300+00$

- a.?. $3,100+0: 1$

- $4: 536300+00$

$.5311 \% 3+00$ -4356300+00 
- $1329760+01$ . $8419 \mathrm{col}+00$ . $9893500+0.3$ - $1336430+01$ - $3364600+00$ - $9927100+00$ - $1343130+01$ . $8309400+00$ - $9954200+00$ - $1349860+01$ . $8253400+00$ . $\$ 974900+00$ - $1356630+01$ . $8196500+00$ . $9989500+00$ $.1363430+01$ $.8138800+00$ $.7997800+00$ $.1370260+01$ . $8080300+00$ - $9999900+00$ . $1377130+01$ . $8021000+00$ - $9975700+00$ - $1384030+01$ . $7 \rightarrow 60800+00$ - $785300+00$ . $1390970+01$ . $7899900+00$ $.9968700+00$ $.1397940+01$ . $7338200+00$ $.9945800+00$ . $1+04950+C 1$ $.7775700+00$ $.9916600+C 0$ $.1411990+01$ $.7712500+0$. - 9881.301$)+(10$ $.1419070+01$ . $7648400+00$ - $9833900+00$ $.1426180+n 1$ . $7583600+(1)$ $.97 .3 ? 200+0)$ $.1433330+01$ $.7518100+01)$ $.9738500+100$ $.1440510+01$ $.7451700+0$ ? . $9678600+0 J$ - $1447730+01$ $.7384720+0$. $.9012430+$. - $1454,90+61$ - $7316900+0$ ) . $3540 \rightarrow 00+00$ . $140223 J+01$
- $1000000+01$ . $7545700+00$ - $1452800+00$ - $1000000+01$ - $764 j 300+00$ - $1205000+00$ - $1000000+01$ $.7739100+00$ -9565000-01 - $1000000+01$

. $7833300+00$

$.7074000-01$

- $1000000+01$

. $7525600+0 C$

.4578000-01

- $1000000+01$

- $8016200+00$

- 2079 COO-01

- $1000000+01$

- $3105000+00$

-.4200000-02

- $1000000+01$ . $8171500+00$

-.2920000-01

- $1000000+01$ . $8277000+00$

-.5418000-01 $.1000000+01$ - $8360300+00$

-.7112.000-01 - $1000000+01$ - $8441000+00$

$-.1040200+00$ - $1000000+01$ - E521100+00

- $1288400+00$ - $1000000+01$ - $3533600+00$

- $1535900+00$

$.1000000+01$

. $9674200+00$

$-.1782500+00$

- 1$) 00000+01$

- $8747900+00$

$-.2027900+00$

. $1000000+01$

- 3313600+00

$-.2272000+00$

$.1000000+01$

- $8383.300+00$

$-.2514700+00$ . $1000000+01$ - $8558000+00$ - . $1599(1)+2)$ - $1000000+u 1$ . $9022700+00$ -. $2595300+00$ - 1$) 00000+01$
- $2811600+00$ . $6.56 ? 2000+00$

- $2859500+00$ . $6443300+00$

- $2907400+00$ - $6.332900+00$

- $2.955200+00$ . $6216100+00$

- $3002400+00$

$.60 \div 7900+00$

. $3050600+00$

- $597.9300+00$

- $3098200+00$

$.5857400+0.0$

. $3145700+00$ . $5735200+00$

- $3193100+00$ $.5011700+00$

- $3240400+00$ - $5436900+00$

. $3227700+00$ - $5360900+00$

- $3334900+00$ . $5233700+00$

- 33 33?0 $00+c 0$ $.5105300+00$

- $3429000+00$

- $4975700+00$

$.3475900+00$

. $4845000+00$

-352? $700+00$ $.4713300+00$

- $3569500+00$ - $4580500+00$

- $3616200+00$ $.4446,500+00$

$.365 .1700+00$ . $4311800+00$

$.37 ?) 7 \% 00+00$
. $9596000+00$

$.908430)+0$ )

-.5396.300+C. 0 . $4175400+60$

$.9582410+00$ . $916 ; 3000+00$

. $5450200+0 ?$ - $3993400+00$

- $9568000+01$

. $9246100+00$

$.556: 30,10+00$ - $3905200+10$

- $955.34 \mathrm{CJ}+0 \mathrm{~J}$

. $9320400+00$

- $5546400+(; 0$

- उiszijo + J0

- $95.3 \times 500+2 ?$

. $9301000+00$

- $57 ?: 77 \cdot 3+1) 0$

$.342+5 ! 0+00$

- $952330 \tilde{u}+(i)$

$.4457810+00$

- $5810400+00$

$.324 \% 30+00$

. 750 ri. $) 0+00$

. $5523 \% 00+0 u$

- $5331+100+00$

. $3053200+00$

$.9402400+00$

$.958 .200+00$

$.9470500+0 J$

. $9635600+00$

$.946 .0400+00$

- $9037200+00$

. $9444100+00$

$.9734300+00$

. $9427500+00$ $.9773600+00$

$.9410300+02$ $.9818500+00$

$.939370 i+00$ $.985450 n+02$

- $9376500+02$ . $5886500+00$

- $9.355030+(i)$ $.9914600+00$

- $9.741300+00$ .90 .3 is $(5)+07$

- 9323303000

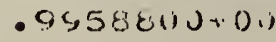

- $9305100+0 i)$ . $99740(0+00$

- $0.81 C 200+00$

$.9: 280000+00$ - $7074000-01$

- 597? ?0u0+00 - ZBE $7200+00$

- $6051700+00$ . $2675000+0$ )

- $\$ 131 \geq 00+00$ - $2481300+00$

- $6.200900+10$ . $2287500+00$

$.6287 \cdot 100+00$ - :0y $2410+00$

- $6365400+00$ - 1 \& jद $400+00$

-6) $44: 320+00$ - $1699700+00$

$.0 .513300+00$ $.1502300+00$

- $6593800+00$ $.1304 .00+00$

. $\epsilon \epsilon 6.8700+00$ $.1105700+00$

$.6742000+00$ - cos $10,111-01$

- $6383200+00$ 
- $7248400+00$ - $9453(00+0 i$ . $1469610+01$ $.7179100+0)$ . $93792 \mathrm{CO}+\mathrm{uU}$ - $1476980+01$ $.7109100+00$ - $9289600+00$ - $1484380+01$ - $7033500+01$ . $9194200+00$ - $1491820+01$ - $6767100+(i)$ . $9093000+00$ . $1499300+01$ . $6395000+\mathrm{i}^{3}$ - $8986100+c 0$ - $15068 ? 0+01$ . $6322200+C$ ) - $3873600+00$ . $1514370+01$ . $6748500+01$ - $3755600+(2$ - $1521950+01$ - c674600+0u - $3632100+00$ - $1522590+01$ $.6597800+00$ - $55032 \mathrm{CO}+0$ ? . $1537260+01$ $.6524403+00$ - $330 \rightarrow \mathrm{COO}+00$ - $1544960+01$ . $(44831)+0)$ - $8229500+00$ . $1552710+01$ . $6371500+60$ - $8385000+00$ . $1560490+01$ $.6294100+00$ $.7935300+C 0$ - $1509310+C 1$ $.6216100+0 u$ $.77807 \mathrm{CO}+00$ $.1576170+61$ $.6137500+00$ - $7621300+00$ . $1584070+01$ $.6058200+C 0$ $.7457100+00$ $.1592010+01$ $.5978300+00$ $.7248200+00$ $.1599490+j 1$ . $5897900+00$ $.7114700+0 j$ $.1603010+01$ $.5816800+00$
- $9086300+00$

$-.3232900+00$

- $1500000+01$

$.9147900+00$

-. $3468400+00$

- $1000000+01$

- $9207500+00$

$-.3701800+00$

- $1000000+01$

. $9265000+00$

-.393290u+00 - $1000000+01$ - $320400+00$

-.4161500+00 - $1000000+01$ . $9373700+c 0$

-.4387500+00 - $1000000+01$. - $9424900+00$

- $4610700+00$ - $1000000+01$ - $9474000+00$

-.4331100+00 - $1000000+01$ - 9521$) 900+00$

-.5043500+0u - $100.000+01$ - $5565700+00$

-.5262700+0.

- $1000000+01$

- $9608400+00$

-.54730600+00

- $1000000+01$

- $9646800+00$

-.5681100+00 - $1000000+01$ - $9087200+00$

-.5885 COO+00 - $1000000+01$ - $972.3300+00$

-.6085300+00 - $1000000+01$ - $9757200+00$

-.6281700+0)

- $1000000+01$

$.9789 \mathrm{CON}^{2}+00$

$-.6474300+00$ - $1000000+01$

- $9818500+00$

-. $66623 \mathrm{CO}+0 \mathrm{u}$ - $1000000+01$

- $9945900+00$

$-.6947100+00$

- $1000000+01$

- $9871000+00$

-. $7027100+00$

- $1000000+01$ $.93 ; 3500+0 u$
$.4175900+01$

- $3755600+00$

. $4037200+00$

- $3801900+00$

. 3901500+00

$.3848100+00$

. $3733000+00$

- $3894200+00$

- $3623500+00$

- $39+0 \geq 00+00$

- $3483400+00$

- $3980100+00$

$.3342+00+00$

-4331300+00

. $3200600+00$

- $4077600+00$

. $3058200+00$

- $4123200+00$

. $2515000+00$

- $4168700+00$

. $2771200+00$

- $4214100+00$

$.2626800+00$

- $4259+00+00$

. $2431800+C 0$

- 431$) 4600+00$

- $2334200+00$

-4.34\%700+00 . $21 ; 0100+00$

- $4304600+n 0$ $.234 .3 .500+013$

$.44 .89500+00$ - $1836400+0$ o

$.4484200+C 0$ - $174: 3900+00$

- $4523 \rightarrow 00+00$ - $1601000+00$

- $4573400+00$ - $1452800+00$
$.99 .37100+00$

- $9203000+00$

- $9405300+00$

- $9240100+00$

- $9535401+00$

- $9230000+00$

- 99(9000+0:

$.9210600+00$

$.5555700+00$

$.9191030+0.3$ - $9537900+0$ ?

$.9171200+0 \%$

. $99712100+00$

- $915120 \mathrm{~J}+0 \mathrm{D}$

- 9serizootou

- $9130900+00$

- $9943400+C ?$

- $911.2400+00$

- $9916.600+00$

$.90897100+00$

- $93888 \div 00+00$

- $9068700+(1)$ - $9857200+60$

- $9047500+01)$ - $9821500+010$

- $9026.10 u+00$ - $978 ? 000+C 0$

- 300450$)+0 \%$ - $97.38350 \%+00$

- $89835(10+0)$ $.9 \in 91100+0.1$

- 89605iu+(1i)

. $963+81 ! n+00$

- $8939200+00$ - 9583700+(1)

$.8915700+00$ - $95258.00+00$

- E632900+C? $.946 .3000+00$
$.5077 .0-01$

$.0 ? E 14) \%+00$

- $3077010-01$

$.7032800+00$

- $1080000-01$

$.7103500+00$

-. $9200000-02$

$.7173+000+00$

$-.2920000-01$

$.724 ? 2,3+00$

- $4 \div 1+500-01$

$.7311300+00$

$-.6515) 30-01$

$.7379 \div 30+c 0$

- $890 y 000-C 1$

$.7445400+00$

-. $1039900+00$

- $7512300+00$

$-.1283400+00$

$.7578400+C 0$

$.148: 6,500+00$

$.764 .3700+00$

- $1034000+00$

$.7707400+100$

$-.1880800+00$

$.7770730+00$

-. $2076100+00$

- $79=3.100+30$

- 2 ? $70(1) 0+00$

- $7835000+0 i$

$-.24 i \cdot 3.10+(10$

$.796 ;(000+00$

$-.205 \cdot 1.19 \mathrm{j}+\mathrm{ij}$

- $80162300+00$ $-.2851: 00+00$

- 80756:0 -. $3043000+00$

- $134200+00$ -.32323(10+1)0 
. $6936500+00$ - $1616070+$ (i 1 $.5735200+00$ - $6754600+c 0$

- $1624180+01$ - $5653000+00$ . $65682 \mathrm{CO}+00$ $.1532320+01$ - $5570200+00$ $.6377600+00$ . $1640500+01$ . $54.96900+00$ $.6183100+00$ - $1648720+01$ . $5403000+00$ - $59847 \mathrm{co}+\mathrm{J}$ - $1048720+01$ . $5318600+00$ . $57826 \mathrm{CJ}+00$ - $1649720+01$ . $5233700+00$ . $55763 \mathrm{Co}+0 \mathrm{~J}$ - $1648720+01$ - j143200+(1) - $5367600+C J$ - $1648720+01$ $.506 ? 200+0 u$ - $5155000+00$ . $1648720+01$ . $4975700+00$ - $4939200+00$ - $1649720+0$ ? - $4883700+00$ $.4720300+00$ - $1048720+01$ $.4801200+00$ - $4498500+0$ ) $.1648720+1$ . $471.3360+00$ . $4273300+00$ - $1648720+01$ . $4624900+0 i)$ - $4046500+00$ - $1648720+01$ $.45 .36000+00$ - $38166,00+i 30$ - $1643720+01$ - $4446600+00$ . $3584400+00$ - $1648720+01$ $.4356800+0 J$ . $3340000+00$ - $1648720+01$ . $4266000+00$ $.3113300+\mathrm{CO}$ - $1645720+01$ $.4175700+30$ - $2874800+00$
-.7202800+00 - $1000000+01$ - $9914600+00$

-.7373900+00 - $1100000+01$ - Sy33000+00

-.75405C0+00 - $1000000+01$ - $9949200+00$

-.77023c0+00 - $1000000+01$

- $9463200+00$

-.7!5.300+00 - $1000000+01$ - $9974900+00$

-.8311400+00 - $1000000+01$ - $998400+00$

- $8153500+00$ - $1000000+01$ - $5491700+00$

-.8300500+00 - $1000000+01$ - $9990701+01$

-.84.37300+00 - $1000000+01$ - SS99400+00

- $8568900+00$ - $10 \mathrm{CO} 000+01$ - 9599900+00

- $8695100+00$ - $1000000+01$ - $9998200+00$

-.8815800+00 - $1000000+01$ - $9994200+00$

- $8931100+00$ - $1000 \mathrm{COO}+01$ - $9987900+00$

-. $9040700+00$ - $1000000+01$ - $9979400+00$

-.9144700+00 - $1000000+01$ - $99637 \mathrm{cot} 00$

- $9243 \mathrm{C00}+00$ - $1000000+01$ - $9955700+00$

-.9335500+00

- $1300000+01$

- $5=40400+00$

- $9422200+00$

- $1000000+01$

- $9923000+00$

-.9503000+00

- $11000000+01$

- 550:3 $300+00$

-. $\$ 577900+00$
. $4617300+00$

. $1304 ? 00+00$

$.4602100+00$

- $1155400+00$

- $4706300+00$

- $1006300+00$

. $4750300+00$

. 8569000-01

$.4794300+00$

. $7074000-01$

$.48 .38100+00$

$.5577000-01$

. $4881800+00$

. 4079000-01

- $49.25 .300+00$

- $2579000-01$

i

- $4908300+00$

- $1080000-01$

$.5012100+00$

-.4200000-02

$.5055300+00$

-. $1920000-01$

- $5098400+00$

$-.3420000-01$

- $3141400+00$

-.491.9000-01

$.5134200+00$

$-.64160100-01$

. 52.26900+0J

-.7912000-01

- 5?6.9400+00 -.9406000-01

$.5311(3)(1)+00$ -. 10 (1)

$.53542 .00+00$ - $12.38800+00$

$.5356 .300+00$ -.134750n+00
- \&3 - $49,900+c 0$

- $9393500+00$

- $8846,700+00$

- $9326200+00$

- $8823300+00$

$.9252100+00$

- $8799700+00$

$.9174400+00$

- $8775800+00$

. $9093000+00$

- $8751700+00$ $.9007900+00$

- $8727400+00$ - 8319330+00

- $370 ?=00+6$ - $30: 71:) u+0$ )

- $807 !=00+00$ . $8731300+00$

- $8653200+00$ - BE $32100+00$

- et $23100+00$

- $8529400+00$

$.860 .740+00$ - $3423300+00$

$.8577100+0 u$ - $8313800+0$ i

- $8551303+00$ $.8201300+00$

- $85.25200+00$ - $8(1+35,000+0 u$

$.849 ; 000+00$ $.790 \% 700+00$

$.5479+31)+31$ $.784 .3 \%)(1+i)$

- $841, ; 0(0+10$ $.771,10)+(1)$

$.841 \div 000+C 0$ - $7583: 3600+00$
. $8191400+0$

- $3421500+00$

- $3248900+00$

- $3608700+00$

- $830500 u+00$

$-.3794500+00$

- $8300300+00$ -. $3978900+00$

$.8414700+00$

$-.416 .1500+C i$

- $84631300+00$

$-.434 .300+00$

- $8521100+30$

$-.4521300+00$

- $357: 1000+00$

$-.40 \div+1(1)+00$

$.8624000+00$

$-.4874800+00$

- $8674200+00$

$-.5048 \div 00+00$

$.872 .3: 00+00$

$-.5220100+00$

$.3772000+0 ?$

-.53 e $9600+c 0$

- ४४ISE0O+CO

$-.5557000+00$

- 3ecisun+on

$-.5782100+00$

- $811: 210)+12$

-.

- $35,57,100+(10$

-.5.) 455010+0.

- $9001000+c: 0$ - $62.031,110+c 0$

$.0044100+10$

-. $3558 \cdot 10+1)$

$.90 \varepsilon(2300+01)$

$-.6512 .700+00$ 
- $1643720+01$ - $40849 \mathrm{CO}+00$ - $2634500+00$ - $1648720+01$ - $3993400+00$ . $2392500+00$ - $1648720+01$ - $3901500+00$ - $2149000+00$ - $1648720+01$ . $3809200+00$ - $1904200+00$ - $1648720+01$ . $3716600+00$ - $1658200+00$ - $16483720+01$ - $3623000+00$ - $1411200+00$ . $1648720+01$ - $3530200+00$ $.11633 \mathrm{Co}+00$ - $1648720+01$ - $3436500+00$ -9140000-01 - $1643720+01$ - $3342400+00$ . $6654 \mathrm{C} \cdot \mathrm{O} 0-01$ - $1640720+01$ - $3248 \mathrm{COO}+00$ - $4158000-01$ - $1648720+01$ . $3153200+00$ - $1659000-01$. - $1648720+01$ - $3053200+00$

$\therefore 3410000-02$ - $1648720+01$ - 2462800+00

-.3340000-01 - $1648720+01$ . $2867200+00$

-.5837000-01 . $1648720+01$ $.2771200+00$

-.8331000-01 $.1648720+u 1$ $.2675001+00$

$-.1082000+00$ $.1648720+01$ . $2578500+00$

$-1330100+00$ $.1648720+01$ . $2481800+00$

-. $15775 \mathrm{cot} 00$ . $1648720+01$ - $2384800+00$

-.1823800+00 - 1 i) $48720+01$
- $1000000+01$

- $5831300+00$

$-.9646700+00$

- $1000000+01$

- $9857200+00$

$-.9709600+00$

- $1000000+01$

- $9330800+00$

$-.9766400+00$

- $1000000+01$

- $9802200+00$

-.9817000+00

- $1000000+01$

$.9771500+00$

-.9861600+00

- $1000000+01$

- $9738500+00$

- $5859900+00$

- $1000000+01$

- $7703300+00$

-. $9432100+00$

. $1000000+01$

- $9665900+00$

-. $\$ 958110+c 0$

- $1000000+01$

- $96.25400+01)$

$-.9977800+00$

- $1000000+01$

- $9584700+00$

-.5931400+00

- $1000000+01$

- $9540900+00$

- $599860 u+00$

- $1000000+01$

. $9494900+00$

-. \$\$\$9600+00

- $1000000+01$

- $94467(i 0)+00$

$-.9994400+00$

- $1000000+01$

- $9376500+00$

-. $5982900+00$

- $1000000+01$

- $\$ \$ 44100+00$

$-.4965200+00$

- $1000000+01$

- $9283600+00$

- . $5441300+00$

- $10 \mathrm{CD} 000+01$

- $9233000+00$

-.9.711100+0u

- $1000000+01$

$.9174400+00$

-. $3674300+00$

- $1000000+01$

- $9113700+00$

-.9832300+00 - $1000000+01$
$.5438300+00$

$-.1535900+60$

- $5480200+00$

$-.1584000+00$

$.5522000+00$

-.1831600+00

$.5563600+00$

-. $1978900+00$

$.5605130+00$

$-.2125700+60$

. $5646400+00$

-. $2272000+00$

$.56376,00+00$

$-.2417300+00$

$.57237 \mathrm{ju}+00$

-.2563100+00

- $3769000+00$

$-.2707300+00$

$.5810400+00$

-. $2851900+00$

$.5351000+00$

$-.2995300+00$

- $5891400+00$

-. $31.38100+00$

- $5931200+00$

$-.328 .1200+00$

$.5972000+00$

$-.3421500+00$

$.6012007+00$

- $35062100+00$

$.6051900+00$

$-.3701800+00$

- $6001000+00$

$-.3840700+00$

$.6131200+00$

-. $3978300+00$

. $6170600+00$

$-.4116000+00$

$.0209400+00$
- $8591 y(10+c 0$ . $7457100+00$

- $8364600+60$

- $7: 22300+00$

- $8337100+00$

$.7134600+0$ ก

- $8309400+00$

$.7044100+00$

- $8281500+0)$

- c900700+00

$.8253400+00$

$.67546,00+00$

. 8225000+00

- $6605+100+00$

$.3146950 \mathrm{c}+\mathrm{vil}$

$.6454300+0$ ?

$.8107700+00$

$.6300300+00$

. $8138800+00$

$.6143700+01$

. $8109600+00$

$.5984700+00$

- $8080300+00$

. 5 ह2 $33300+00$

$.8050700+00$

$.5059600+00$

$.8021000+00$

$.5493600+c 0$

- $7991000+00$

$.5325300+00$

$.7904800+00$

$.5155000+00$

- 7531$) \leq(10+00$ . $4982600+00$

$.7899900+00$ $.4808200+00$

- $7 म 69200+00$ . $4631900+00$

$.7338200+00$
- $912760.0+00$

$-.6662300+00$

- $9165700+00$

- $681 \mathrm{u} \times 00+00$

- $9207500+00$

- $6 \cos 55000+00$

$.9246100+c 0$

$. .7097 .300+00$

- $9283700+00$

$-.7237400+00$

$.9320400+\mathrm{C} 0$

$-.7 \because 73900+0$ )

- $9356200+00$

-.75075010+00

- $92 \subseteq 1(10 i)+00$

- . 76 $35200+00$

$.4424900+00$

$-.7765700+00$

. $9457800+00$

$-.7890100+00$

. $9489300+00$

$-.8011400+00$

- $9520.300+60$

-.8129500+00

$.9531000+00$

-. $8244400+00$

- $9580 ? 60+00$

$-.8355400+00$

- 960 E $400+00$

- E454100+00

- ce. $35600+00$

-. 356 is $700+00$

- $=651: 10+00$

- ec $70300+00$

- cse. $37200+1 ; 0$

- $8769200+00$

- $9711,00+10$

-. $8862000+00$

$.9734300+00$ 
- 22 ล $7500+00$

-. $2069 \mathrm{COO}+00$ - $1648720+01$ . $2190100+00$

$-.2312900+00$ - $1648720+01$ - $2092400+00$

-.255540J+00 - $1648720+01$ - $1994500+00$

$-.2796300+00$ $.1648720+01$ - $1896400+00$

-. $3035400+00$ $.1643720+01$ $.1793100+00$

$-.3272600+00$ - $1648720+01$ . $1599700+00$

-. $35078 \mathrm{CO}+00$ - $1 \epsilon 48720+01$ . $1601000+00$

$-.3740900+00$ - $1648720+01$ . $1502300+00$

-. $3971500+J u$ - $1648720+01$ - $1403300+00$

$-.41913700+00$ - $1648720+01$ - $1304200+00$

-.4425200+00 . $1643720+01$ - $1205000+00$

-.4648 C CO+00 . $164.3720+01$ . $1105700+00$

-.48674CO+OO - $1643720+01$ - $1006300+00$

-.5084700+00 - $1648720+11$ . $9067000-01$

-. $5298400+00$ . $1648720+01$ . $8071000-01$

$-.5508700+00$ . $1648720+01$ . $7074000-01$ -.5715600+00 . $1643720+01$ - $E U 76,000-01$

- .5) $1.35, \mathrm{C} 2+00$ - $1 t 4$ t3 $72(1+1) 1$ - 5077)00-01

-.611860u+00 . $16483720+01$ $.4079000-01$
- $9050900+00$

$-.9783600+00$

- $1000000+01$

- $8586100+00$

-.9728800+00

- $1000000+01$

- $8919300+00$

-.9668000+00

- $1000000+01$

- $8850500+00$

-.9601100+00

. $1000000+01$

- $8779600+00$

$-.9528200+00$

- $1000000+01$

- $87068 \mathrm{co}+00$

-.9449300+00

- $1000000+01$

- $8632100+00$

$-.9364600+00$

- $1000000+01$

- $8555400+00$

-.9274000+00

- $1000000+01$

- $8476800+\mathrm{Cu}$

-.';177500+00

- $1000000+01$

- $3396300+00$

-.9075400+00

- $1000000+01$

- $831333130+00$

-.8\$67600+00

- $1000000+01$

- $822950.0+00$

-.9354200+00

- $1000000+01$

. $8143400+00$

$-.8735200+00$

. $1000000+01$

- $8055400+00$

$-.8610800+00$

- $1000000+01$

- $7965700+00$

$-.8481000+00$

- $1000000+01$

- $78741(0+00$

- $3.345900+00$

- $1000000+01$

$.7783700+00$

-. E2.05600+00

- $1000000+01$

- 7 C85600+00

-.3060200+00

- $1000000+01$

- $7383900+00$

- $7909700+00$

- $1000000+01$

$.7450300+00$
$-.4252200+00$

-624400nt 00

-.4337500+00

$.6287 \rightarrow 00+00$

-.4521:300+00

$.6326700+00$

-.4655000+00

$.6365400+00$

$-.4787300+00$

$.6403900+00$

$-.4918400+00$

$.6442200+00$

$-.5048500+00$

$.6480300+00$

$-.5177400+00$

$.6518300+00$

$-.5305100+00$

$.6556200+00$

$-.5431700+00$

$.6593400+00$

$-.5557000+00$

$.6631400+00$

$-.5681100+00$

$.6668700+00$

$-.580 .3900+00$

$.6705900+00$

$-.5925400+00$

$.6742900+00$

$-.6045500+00$

$.6779700+00$

$-.6164300+00$

$.6816400+00$

$-.62811700+00$

$.6852900+00$

$-.6397700+00$

.1,880200+00

$-.6512300+00$

$.6925400+00$

$-.6625400+00$
$.4453700+00$

$-.8+534 \Omega 1+0 !$

. $7307100+00$

- $5757: 000+00$

. $4273300+00$

-.9040700+0u

$.7775700+00$

. $4052100+0$ )

- $97786 \mathrm{C} .0+00$

$-.9124407+0)$

. $7744200+00$

. $3908800+00$

. $9709100+00$

-. $9204400+00$

$.7712500+00$

- $7814500+00$

$.3724000+00$

$-.9280700+00$

$.7680500+00$

- $3837000+00$

$.3537600+00$

. $7648400+00$

. $3349900+00$

- $7 \in 16100+00$

$.3100800+00$

. $7583600+c 0$

- $29704 i j+00$

$.7550900+00$ $.2773900+00$

- $7518100+00$ . $2536200+00$

$.7485000+00$ $.2392500+00$

$.7451700+00$ $.2157800+00$

$.7418300+00$ $.2002300+00$

- 7334700+ 00 - 1 bide. $000+00$

$.7350900+00$ $.1608930+00$

. $7315700+00$ $.1411200+00$

- $72832700+00$ - $1212900+00$

$.7246400+00$ $.1014200+00$

$.721380 .7+00$ $.81500 \mathrm{CO-O1}$
-.0 $3(333)+00$

$.9354500+00$

-.94Z2200+00

. $9071000+00$

$-.44 t .7302+n 0$

- $3886500+00$

$-.954 \varepsilon(1))+00$

- $9901000+00$

$-.9606100+00$

$.9914600+00$

$-.9659800+00$

- 9427100+00

-.97096.00 +00

- $3538300+00$

-.9755500+00

$.094 \div 200+00$

-. $790750.1+00$

$.9 .758 .30 .0+00$

$-.98 .35600+00$

. $9967400+C 0$

-. $9899700+60$

- $3974900+0 J$

-. $\cdot 3(13900+(1)$

- c) $1500+00$

-.9926200+0u

$.99810 u+u 0$

$-.994 \leqslant 40 c+10$

- $9921760+c 0$

-.9966700+10 
$-.6314400+00$ - $1648720+01$ $.3079000-01$

$-.6506300+00$ - $1643720+01$ . 2079000-01

-.6694000+00 - $1648720+01$ - $1080000-01$

-.6877700+00 - $1648720+01$ .8000000-03

-.7057000+00 - $1648720+01$

-.9200000-02

-.7231900+00 $.1648720+01$

-.1920000-01

-.7402300+00 - $1648720+01$

-.2920000-01

-.7568000+00 . $1645720+01$

-.3919000-01

-. $7729100+00$ - $1648720+01$

-.4913000-01

- $7885300+00$ - $1643720+01$

-.5917000-01

-.8036500+00 - $1648720+01$

- .0y $15000-01$

-. $182800+00$ - $1648720+01$

- .7912000-01

- $3323 \rightarrow \mathrm{CO}+0 \mathrm{O}$ . $1648720+01$ $-.3909000-01$

-.8459800+00 - $1648720+01$

- .9904000-01

- $35,905 \mathrm{co}+00$ - $1 \epsilon 48720+01$

-. $1039900+00$

$-.3715800+00$ . $1648720+01$

- $11892.00+00$

-.3835600+00 - $16+8720+01$

- $1288400+00$

-.894:3900+00 - $1648720+01$

-.1337500+00

-.9058600+00 . $1648720+01$

-.1436500+00

-.91617CO+00
$-.7754300+00$

- $1000000+01$

$.7390100+00$

$-.7594000+00$

- $1000000+01$

- $7288200+00$

$-.7429000+00$

- $1000000+01$

$.7184600+00$

-.7259300+0u - $1000000+01$ - $7079500+c 0$

$-.7085100+00$ - $1000000+01$ $.6972800+00$

-.6906500+00 - $1000000+31$ $.636+500+00$

-.6723600+00 - $1000060+01$ . $6754600+00$

$-.65364: 0+0)$ - $1000000+01$ $.6643360+00$

$-.6345200+00$ - $1: 00000+01$ - $0530400+00$

$-.6150000+00$ - $1000000+01$ - $64161 \mathrm{cot}+0$

-.5951000+00 . $1000000+01$ . $6300300+00$

$-.5748200+00$ - $1000000+01$ - $6183100+00$

-. $5541+00+00$ - $1000000+01$ - $0064510+00$

$-.5332100+00$ - $1000000+01$ - $5944,000+00$

$-.5119400+011$ - $1000000+01$ . $582.3300+00$

-. $4902000+00$

- $1000000+01$

- $5700760+00$

-.4683200+00

- $1000000+01$

- $5576300+00$

-.44603Cn+00 - $1000000+01$ - $5451700+00$

- $4235300+00$

- $1000000+01$

. $5325300+00$

-.4008000+0u
- $961400+00$

$-.6737600+00$

$.6997200+00$

$-.6847100+00$

- $7032800+00$

$-.6955600+00$

- $7068300+00$

$-.7062600+00$

. $7103500+00$

$-.7168000+00$

- $713: 3600+00$

$-.7271800+00$

$.7173600+00$

$-.7373 .00+00$

$.7208300+00$

$-.7474400+00$

- $7242900+00$

-.7573200+00

$.7277300+00$

$-.7670300+00$

$.7311500+00$

$-.7765700+00$

$.7345500+00$

-. $7859300+00$

$.7379300+00$

$-.7951200+00$

$.7413000+00$

$-.8041300+00$

. $7446400+00$

$-.8124500+00$

$.7477700+00$

-.8216000+00

$.7512300+00$

$-.8300500+00$

$.75457 .20+00$

- .8383300+00

$.7578400+00$

-.840+100+00
$.7179100+00$

. $6155000-01$

$.7144200+00$

- $41580 \cup 0-01$

- $7109100+00$

. $2159000-01$

. $7073900+00$

. 1590000-02

$.7038500+00$

-.1341000-01

. $7002800+0.1$

$-.384 .0000 \div 01$

$.6967100+00$

$-.5337000-C_{1}$

$.6931100+00$

-.7833000-01

$.6895000+00$

$-.9825000-01$

- $6: 3583700+00$

-.1181300+00

$.6822200+00$

$-.1379700+00$

- $6785600+00$

$-.1577500+00$

- $6748800+00$

-. $1774600+00$

. $6711300+00$

$-.1971100+00$

- $6674 E 00+00$

$-.2166300+00$

- Et $37300+00$

-. $2361600+00$

$.654 \cdot 300+00$

$-.2555400+00$

.6562:00+00

-. $2743200+00$

$.6524400+00$

$-.2940000+00$
- $9955360+00$

-.9981000+00

. $9977300+00$

$-.9591400+00$

- $9909400+00$

$-.9997700+00$

- $1000000+01$

$-.1000000+01$

- S9c9t $90+00$

- .

- $998200+03$

-.9992600+0?

- ?795700+00

- . g.E2 $700+0 J$

- $5992.3(10+00$

$-.796530 .1+00$

- $9787.100+00$

$-.9951600+C$ )

- $913(32530: 1+00$ $-.00 .30000+00$

$.8976160+00$

$-.69(0440)+0)$

- $) 3(3700+0)$

$-.4374300+00$

- $9960200+00$

-. $9841300+00$

$.90701300+00$

$-.9303300+00$

- $594(1400+00$

-.1762400+10

- $9029000+00$

$-.7717 ? 0 n+n 0$

- $49161,0 n+0)$

- grecoscoto

- $990: 3$ iciotio

$-.96 .14100+00$

- $98.880 n 0+c .7$

-. $9558100+00$ 
- $16+8720+01$ -.1585300+00

-.9259c00+00 - $1648720+01$ - $1684000+00$

-.9350500+00 - $1648720+01$

-.1782500+00

-.9436200+00 $.1643720+01$

- $1880800+00$

-. $9516000+00$ - $1648720+01$

-.1978900+00

-.9589900+00 - $1643720+01$

-.2076800+00

-.9657700+00 . $1648720+01$

$-.2174500+00$

$-.9719600+00$

$.1648720+01$

-.2272000+00

-.9775300+00

- $1648720+01$

-.2369300+00

-.9824900+00

$.1648720+01$

$-.2466300+00$

-. $9868400+00$

- $1648720+01$

-.2563100+00

$-.9905800+00$ $.1648720+01$

-. $2653600+00$.

-.9936500\$00 . $1648720+01$

-. $2755900+00$

$-.99618 \mathrm{CO}+00$ - $1648720+01$

-.2851900+00

-.9980500+00

. $1648720+01$

-.2947600+00

-.999.3000+00

. $1548720+01$

-. $3043000+00$

-.39 $792 \mathrm{CO}+0 \mathrm{O}$

$.1648720+01$

-.3138100+00

-.9993200+00

- $1648720+01$

-.3232900+00

-.7942900+00 - $1648720+01$

-.3327400+0u

-.3983400+00 . $1648720+01$
$.1000000+01$ . $5197800+00$

-. $3777700+00$

- $1000000+01$

. $506,100+00$

$-.3545100+00$

- $1000000+01$

- $4939200+00$

-. $.3310200+00$

- $1000000+01$

- $48082(\mathrm{~s})+00$

$-.3073300+00$

- $1000000+01$

. $4676200+0 J$

-.2834500+0u

- $1000000+01$

- $4543100+00$

-.2593900+00

- $1000000+01$

- $4408900+00$

-. $2351700+00$

- $1000000+01$

- $4273800+00$

$-.2108000+00$

- $1000000+01$

. $4137700+00$

$-.1862900+00$

- $1000000+01$

$.4000700+00$

- 1 C $16800+00$

- $1000000+01$

- $3362800+00$

- $1364600+00$

- $1000000+01$

- $3724000+00$

$-11121500+00$ - $1000000+01$ - $3584400+00$

-.8723000-01

- $1000000+01$

$.3443900+00$

-.6235000-01

- $1000000+01$

- $3302700+00$

-.3738000-01

- $1000000+01$

$.3160800+00$

$-12.39000-01$

- $1000000+01$

$.3018100+00$

- $1261000-01$

- $1000000+01$

- $2874800+00$

- 3760000-01

- $1000000+01$

- ?730800+00

-6257000-01

$.1000000+01$
. $7611000+00$

$-.8543000+00$

$.7643300+00$

$-.8620000+00$

$.7675400+00$

-. $8695100+00$

- $7707400+00$

-.87682jo+00

$.7739100+00$

-. $8339300+00$

$.7770700+00$

-.8908500+00

- $7802100+00$

-.8975600+00

$.7833300+00$

$-.9040700+00$

$.7864300+00$

$-.910 .3300+00$

$.78 y 5000+00$

$-.9164300+00$ .

$.7925600+00$

$-.922390(j+00$

- 7956,000+00

$-.923 .0700+00$

$.7986200+00$

$-.9335500+00$

$.8016200+00$

-.9388300+00

. $8046000+00$

$-.9438900+00$

- $80756,00+00$

-. $9487300+00$

$.8105000+00$

$-.9533700+00$

$.8134200+00$

$-.9577900+00$

. $8163100+00$

$-.9619900+00$

. $3191900+00$
. $6436400+00$

$-.3130500+00$

$.6448300+00$

-. $3319900+00$

$.6410000+00$

$-.3507800+00$

$.6371500+00$

-. $3694400+00$

- $6332900+00$

$-.3879500+00$

- $0294100+00$

$-.4063100+00$

$.6255200+00$

-.42450Co+ 00

$.6216100+00$

$-.4425200+00$

$.6176900+00$

- $4603700+00$

$.6137500+00$

$-.4790300+00$

$.6097900+00$

$-.4955000+00$

$.6058200+00$

$-.5127700+00$

$.6018300+00$

$-.5293400+00$

. $5978300+00$

$-.5466900+00$

$.5933200+00$

$-.5633300+00$

- $\$ 897900+00$

$-.5797400+00$

$.5857400+00$

-.5959200+co

- $5816800+00$

$-.6118600+00$

$.5776100+00$

$-.6275530+00$

$.57 .35200+00$
- $9873500+00$

$-.9497400+00$

$.9857200+00$

$-.9432400+00$

- $9839900+00$

$-.9364600+00$

- $982.1500+00$

$-.92925100+0 n$

- मसं ? $200+00$

-. $9216800+00$

- $9732000+00$

-.9137400+00

- $4760700+00$

$-.9054 .320+00$

$.9738500+00$

-. $8967600+00$

$.9715300+00 k$

-. $8877300+00$

- $9691100+00$

$-.8783500+00$

$.9665700+00$

-. $.6 .36100+00$

- $9639800+00$

-. $8585300+00$

$.9612800+00$

- $8481000+00$

- $9584700+00$

$-8373300+00$

$.9555700+00$

$-.82 t, 2300+00$

- $9525.300+00$

-. $3148000+00$

$.9494900+00$

-. $30.30500+00$

- $4463000+00$

$-.7909700+00$

$.9430200+00$

$-.7785700+00$

$.9336500+00$ 


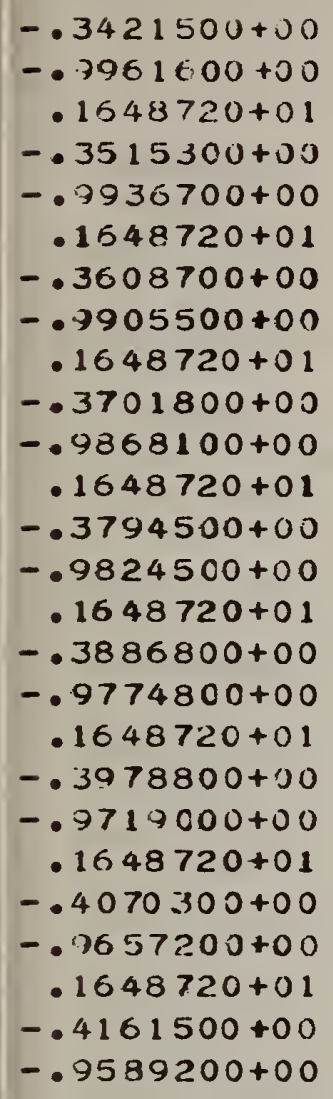

$.2586200+00$

- 8750000-01

$.1000000+01$ . $2441000+00$

$.1123700+00$ - $1000000+01$ - $2295300+00$

$.1371800+00$

- $1000000+01$ . $2149 \mathrm{C0}+00$

$.1619000+00$

$.1000000+01$

$.2002300+00$

- $1865100+00$

$.1000000+01$

. $18551 \mathrm{cot} 00$

$.2110100+00$

$.1000000+01$

$.1707500+00$

$.2353800+00$

$.1000000+01$

- $15 x 500+00$ $.2536000+00$

$1000000+01$ $.1411 .200+00$ $.2836000+00$
$-.9659800+00-.6430000+00$

$.8220500+00 \quad .5694200+00$

$-.9697500+00-.6531900+00$

. $824.3900+00$

$-.5733000+00$

$.8277000+00$

$-.9766400+00$

$.8305000+00$

$-.9797500+00$

$.8332700+00$

$-.9826400+00$

. $8360300+00$

-. $9853100+00$

- $8337600+00$

-. $9 \varepsilon 77600+00$

$.8414700+00$

$-.9899900+00$
$.5653000+00$

$-.6731100+00$

$.5611700+00$ $-.6877700+00$

$.5570200+00$

$-.7021500+00$

. $5528600+00$

$-.7162500+00$

$.5486900+00$

$-.7300600+00$

$.5445000+00$

$-.7435800+C 0$

$.5403000+00$

$-.7568000+00$
$-.7653700+00$

$.9361300+00$

$-.7528500+00$

. $9325200+00$

$-.7395400+c 0$

$.9289000+00$

$-.7259300+00$

$.9252100+00$

$-.7120300+00$

$.9213700+00$

$-.6 .978500+00$

$.9174400+00$

$-.6333 .300+00$

$.9134100+60$

-.6630500+00

$.9093000+00$

$-.6536400+00$ 


\section{HP25: GENERAL DATA FITTING FRS:HLEM}

$$
155
$$

$.7000000+01$

- $4000000+01$

- $4000000+01$

$.1300000+02$

$.2000000+01$

$.1200000+02$

$.7000000+01$

- $1100000+02$

. $7000000+01$

- $2000000+01$

- $7000000+01$

- $1000000+01$

- $3000000+01$

- $2000000+01$

- $3000000+01$

. $60 \mathrm{c0000+01.}$

- $5000000+01$

- $3000000+01$

- $1000000+01$

- $7000000+01$

$.4000000+01$

$-.2000000+01$

$.1000000+01$

-.4000000+01

- $6000000+01$

-.2000000+01

. $6000000+01$

- $9000000+01$

. 0000000

. 00.00000

\begin{tabular}{|c|c|c|c|}
\hline $.5000000+01$ & $.3000000+01$ & $.4000000+01$ & $-120(0001)+12$ \\
\hline $.9000000+01$ & $.7000000+01$ & $.3000000+01$ & - $1919(31)+32$ \\
\hline $.6000000+01$ & $.6000000+01$ & .0000000 & $.12001000+02$ \\
\hline $.9100000+01$ & $.9000000+01$ & $.7000000+01$ & $.2500000+02$ \\
\hline $.3000000+01$ & .0000000 & $.1000000+01$ & $.4000200+01$ \\
\hline $.8000000+01$ & $.1000000+01$ & $.8000000+31$ & $.1700000+02$ \\
\hline $.1000000+01$ & $.4000000+01$ & $.8000000+01$ & - $13000(1)+$,0 ? \\
\hline .0000000 & $.9000000+01$ & $.3000000 \mathrm{Fol}$ & $.1210007+02$ \\
\hline $.3000000+01$ & $.1000000+01$ & $.10000 .00+01$ & $.5000001+01$ \\
\hline $.6000000+21$ & $.7000000+01$ & $.60010000+01$ & $.150012(i)+4 ?$ \\
\hline $.6000000+101$ & $.1000000+01$ & $.9000000+01$ & $.1000: 011+02$ \\
\hline .0000000 & $.4000000+01$ & $.8000000+01$ & $.12000 n)+c 2$ \\
\hline .0000000 & $.5000000+01$ & $.7000000+01$ & $.1200000+02$ \\
\hline $.7000000+0.1$ & $.3000000+01$ & $.2000000+01$ & . 12:00000+02 \\
\hline $.5000000+01$ & $.4000000+01$ & $.3000001)+01$ & $.1300000+02$ \\
\hline
\end{tabular}


HP26: POLYAEMIAL APPROXIMATICN PROELEM

\section{1}

.0000000

.0000000

$.2041000-01$

.0000000

$.4167000-01$

.0000000

$.633 .3000-01$

$.1000000-04$

. 36:95000-01

$.4000000-04$

$.1111000+00$

$.1000000-03$

$.1363400+00$

. $2100000-03$

$.1627300+00$

. $3800000-0.3$

$.1903500+00$

$.6600000-03$

. $2192800+00$

- $105 \mathrm{C} 000-02$

. $2496000+00$

$.1600000-02$

$.2813900+v 0$

.2340000-02

- $3147400+00$

. $3320000-02$

$.3497500+00$

. $4570000-02$

- $3865000+00$

. $6150000-02$

. $4251000+00$

-8100000-02

- $4656500+00$

. $104900 i)-01$

$.5082700+00$

. $1336000-01$

$.5530500+00$

- 168C000-01

. $001200+00$

$.2085000-01$

.645,6000+00

. $2560000-01$

$.7016000+00$

. $311200 n-01$

$.7562600+00$

. $3748002-01$

. $8137100+00$

$.4477 \mathrm{CO0} 01$

. $8740800+00$

. $5308000-31$

$.9375000+00$

- $625 \mathrm{cc000-01}$

- $1004120+01$

- $7312000-01$

$.1074090+01$
$.1000000+01$. c coonou

$.1000000+01 \quad .2000000-01$

$.1000000+01 \quad .4000000-01$

$.1000000+01 \quad .6000000-01$

$.1000000+01 \quad \cdot 3000000-01$

$.1000000+01$

$.1000000+01$

$.1000000+01$

$.1000000+01$

$.1000000+01$

$\cdot 1000000+01$

$.1000000+01$

$.1001000+01$

$.1000000+01$

$.1000000+01$

$.10 \cos 00+01$

$.1000000+01$

$.1000000+01$

$.1000000+01$

$.1000000+01$

$.10013000+01$

$.1000000+01$

$.1000000+01$

$.1000000+01$

$.1000000+01$

$.1000000+01$

$.1000000+01$

$.1000000+01$

$.1000000+00$

$.1200000+00$

- $1400000+00$

$.1600000+00$

- $1800000+00$

$.2000000+00$

$.2200000+00$

$.2400000+00$

$.2600000+00$

$.2800000+00$

$.3000000+00$

$.3200000+00$

$.3400000+00$

$.3000000+00$

$.3800000+00$

$.4000000+00$

$.4200000+c 0$

$.4400000+00$

$.4600000+00$

$.4800000+00$

$.5000000+00$

$.5200000+00$

$.5400000+00$
.0000000

$.4000000-0.3$

$.1600000-02$

$.3600000-02$

$.6400000-02$

$.1000000-01$

$.1440000-01$

$.1500000-01$

$.2560000-0$ !

$.324 \sin 0-01$

$.4000000-01$

$.4840000-01$

$.5760 \cdot 100-01$

. $6760000-01$

$.7840000-01$

$.9000000-01$

$.1024000+00$

$.1156000+c 0$

$.12960000+00$

$.1444000+00$

$.1000000+00$

$.1764000+00$

$.1936000+00$

$.2116000+00$

$.2304000+00$

$.2500000+00$

$.2704000+00$

$.2916000+03$
- .) 190)

$.10 \div 320-104$

$.60000 r 0-04$

$.2200030-103$

$.51001) \bar{ن}(1)-(.3$

$.1000000-02$

$.1730000-02$

$.2740000-0 ?$

$.41300 j 0-0{ }^{\prime}$

$.533519 \cdot 9-9 ?$

- $920001: 1-32$

- $1(005101-01$

$.1 .3 \times 3.0000-01$

. $1758000-01$

-21350w-01

$.2700) 01001$

$.3277901-01$

$.39300(10-01$

-46ur $000-01$

$.54370(1)-01$

$.400000-11$

$.7407000-01$

- $851: 3000-01$

$.5734 x(0-01$

.1105930100.

$.1230100+00$

$.1406100+00$ 
. $3503000-01$

$.1147560+01$

- 9834000-01

- $1224630+01$

- $1131600+00$

$.1305600+01$

- $1296000+00$

. $1390490+01$

- $1477600+00$

- $1479520+01$

- $1677700+00$

$.1572840+01$

$.1897500+00$

. $1670 € 50+01$

. $2133100+00$

$.1773100+01$

$.2401000+00$

$.1880390+01$

$.2637400+00$

- $1932 n 90+01$

$.2993700+00$

- $2110200+01$

- $3336200+0 u$

$.2233100+01$

$.3701500+00$

$.2361600+01$

$.4096000+00$

$.2495890+111$

$.4521200+00$

$.2636180+01$

$.4978700+01$

. $2732660+01$

- $5470100+00$

- $2935570+01$

$.5997000+00$

$.3095100+01$

$.6561000+00$

$.3261480+01$

$.7163+00+01)$

$.8434 .330+01$

. $7807500+0 J$

. उE $15680+01$

$.8493500+00$

. $380.3360+01$

- $9223700+00$

. $9000000+01$

$.100000)+01$

\begin{tabular}{|c|c|c|c|}
\hline $.1000000+01$ & $.5600000+0 u$ & $.3136000+00$ & $.1756200+00$ \\
\hline $.1000000+01$ & $.5800000+00$ & $.3364000+00$ & $.16,51100+00$ \\
\hline $.1000000+01$ & $.6000000+00$ & $.3600000+00$ & $.2160000+00$ \\
\hline $.1000000+01$ & $.6200000+00$ & $.3844000+00$ & $.23833300+00$ \\
\hline $.1000000+01$ & $.6400000+00$ & $.409 r_{3} 000+00$ & $.2621400+00$ \\
\hline $.1000000+01$ & $.6600000+00$ & $.43560(10+i 31)$ & $.287560 c+00$ \\
\hline $.10 .00000+01$ & $.6800000+00$ & $.4624000+00$ & $.31+4300+110$ \\
\hline $.1000000+01$ & $.7000000+00$ & $.4900000+00$ & $.3430000+00$ \\
\hline $.1000000+01$ & $.7200000+00$ & $.5134000+00$ & $.5732500+00$ \\
\hline $.1000000+01$ & $.7400000+00$ & $.547 E 000+00$ & . $4052200+00$ \\
\hline $.1000000+01$ & $.7600000+00$ & $.5770000+00$ & . 4.39110010+cכ \\
\hline $.1000000+01$ & $.7800000+00$ & $.6084000+00$ & $.47+5550)+00$ \\
\hline $.1000000+01$ & $.80000010+00$ & $.6400000+00$ & $.51 \geq 0,71+00$ \\
\hline $.1000000+101$ & $.8200000+00$ & $.6724000+00$ & $051.370 .0+0$ \\
\hline $.1000000+01$ & $.8400000+00$ & $.7056000+00$ & $.5927000+00$ \\
\hline $.1000000+01$ & $.8600000+00$ & $.7346000+00$ & $.0360610+00$ \\
\hline - $1000000+01$ & $.8800000+00$ & $.7744000+00$ & $.601+.00+00$ \\
\hline $.1000000+01$ & $.9000020+00$ & $.8100000+00$ & $.7270000+(10$ \\
\hline $.1000000+01$ & $.9200000+00$ & $.8464000+00$ & $.77 .36900+00$ \\
\hline $.1000000+01$ & $.9400000+00$ & $.8836,000+00$ & . $.305900+00$ \\
\hline $.1000000+01$ & $.9600000+00$ & $.9216,000+00$ & - $\therefore 8.47+00+00$ \\
\hline $.1000000+01$ & $.9800000+00$ & $.9604000+00$ & $.0411300+00$ \\
\hline $1000000+01$ & $.1000000+01$ & $.1000000+01$ & $.1000300+01$ \\
\hline
\end{tabular}


HP27 : SPLINE FITIING PRO $3_{L}$. I

726

$.1180452+05$

-. $1751000+00$ . $1225520+05$

-. $7242000+00$ - $1015648+05$

$-6202000+00$ - $3076900+04$

$-.6127000+00$ - $1058814+05$

-.1142400+J1 $.1103514+05$

-.1610200+01 - $1459502+05$

-. $2033100+01$ -91945.20+04

- $13+3300+01$ -98J1960+vi

-. $1679100+01$ . $8655551+04$

-.1635300+01 - $2394985+05$

-.473.3900+01 $.3273160+04$

-. $1705500+01$ - $1220925+05$

- :672900+01 $.1278816+05$

- $3016600+01$ - $9497280+04$

- .225 $300+01$ - $1283555+U j$

$-.3069400+01$ - $C_{2} E \geq 2410+04$

-.2316400+01 - $1292112+05$

-.3079700+01 $.1495490+05$

-.3575500+01 $.1239504+03$

-.2931600+01 - $1205200+015$

$-.2780000+01$ $.9295520+04$

-.20E1BU0+01 - $2143764+05$

-.4614700+01 . $1210638+05$

-.2502600+01 - $1956311+.55$

-. $3682100+01$ . $2009748+0 ؛ 5$

$-.3204600+01$ - $162037 ?+05$

-.?449400+01 $.1682500+05$
- $2034333+04$ - 35 c0u00-02 - $1430340+04$ - $1610000-01$ $.1653324+04$ - $138 \mathrm{CCO} 0-01$ - $1275220+04$ - $1360000-01$ - $1605340+04$ - $2540000-01$ - $1+558,32+04$ - $3580300-01$ . $1+3.5520+0$ + - $4510000-01$ . $1105723+04$ . $3070000-01$ $.1071334+0.2$ . $3730000-01$ - $4535277+03$ - $3630000-01$ - 2251153+04 - $1053 \mathrm{crotco}$ - $7401413+03$ - 379 criso-01 - $5766060+J 3$ -5940000-01 - 8 638J1+0.3 . 6700000-01 . $5998172+03$ - 5010000-01 . $7023817+03$ . 5820c00-01 - $4585070+03$ . 5150000-01 . $5673120+03$ . 6840000-01 $.65863 .36+0.3$ . 7550000-01 $.5023830+0.3$ $.6510000-01$ $.4137553+03$ . 6180000-01 . $2064141+03$ . 4580000-01 . $5553785+03$ . $1025(00+01)$ - $2804619+03$ - 5560000-01 - $3570743+03$ - $8180 \mathrm{CON}-01$ - 2755 t. $30+03$ - $7320000-01$ - $1359020+03$ . 5440000-01 . $1063428+03$

\begin{tabular}{|c|c|c|}
\hline $.9382400+02$ & $-.3322230+02$ & $.3100320+01$ \\
\hline $.3843003+03$ & $-.1531119+0 \%$ & $.131: 3) \cup c+0 ?$ \\
\hline $.3334053+03$ & $-.1354105+03$ & $.11 ? 877)+0 ?$ \\
\hline $.3300043+03$ & $-.1337830+03$ & 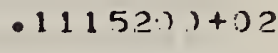 \\
\hline $.6183019+03$ & $-.2494156+03$ & $.2079100+1 ?$ \\
\hline $.03 .9923+03$ & $-.3515550+03$ & $.293 .5 ? u+0 ?$ \\
\hline $.1110754+04$ & $-.443242 .3+03$ & $.35: 34410+.32$ \\
\hline $.76050 .30+03$ & $-.3021170+03$ & $.251 \& 412+0 ?$ \\
\hline $.93253500+0 ?$ & $-.30 \cos 112+03$ & $.31356: 3.30+12$ \\
\hline . $720.3012+03$ & $-.3570425+v=$ & $.23702 t u+0 i$ \\
\hline - $2536 \cdot 3+35+04$ & $-.10346+13+1+$ & - $3622436,1+1) 2$ \\
\hline $.97163 \cup 17+03$ & $-.3723654+i) .3$ & $.316 .4)(0 t) ?$ \\
\hline $.1555925+04$ & $-.5035622+1) 3$ & $.45645 .00+1.2$ \\
\hline $.1316288+04$ & $-.65836204+03$ & $.5490130+02$ \\
\hline - $1375662+04$ & $-.492312 .8+0.3$ & $.4103860+02$ \\
\hline . $1329412+04$ & $-.6701556+03$ & $.5536340+02$ \\
\hline $.1508344+04$ & $-.5057448+05$ & $.42 .153 .30+12$ \\
\hline $.2046056+04$ & $-.6724078+0.3$ & $.5605110+102$ \\
\hline $.2375436+04$ & $-.7306540+03$ & - $(50 ? 44)+0 ?$ \\
\hline $.1990679+04$ & $-.640055 ?+03$ & $.5335420+02$ \\
\hline $.193528 .3+04$ & $-.6069558+0.3$ & $.5059510+1 ?$ \\
\hline $.15652 .37+04$ & $-.4501505+0.3$ & $.3752+70+0$ ? \\
\hline $.36306983+04$ & $-.10075533+04$ & $.8394+443+02$ \\
\hline $.2048336+04$ & $-.5403952+0.7$ & $.4554710+0 ?$ \\
\hline $.3316434+04$ & $-.8039215+03$ & $.6 ? 71300+0 ?$ \\
\hline $.3360025+04$ & $-.71932 .11+0$ & - $64,6340+1 ?$ \\
\hline $.2699596+04$ & $-.5347814+03$ & $.4457 .370+02$ \\
\hline $.27 \% 9749+04$ & $-.5060059+03$ & $.4 ? 130(0+0 ?$ \\
\hline
\end{tabular}


$-.2317600+01$ - $1653760+05$

-.1726500+01 - $1504900+05$

- $1002400+01$ $.3437580+05$

-.1552900+01 $.141649 ?+05$

-.3315 v0u+00 $.2125410+05$ . $219280 n+01$ . $2024304+05$ - $2085500+01$ $.1816080+05$ $.4345300+01$ . $1282688+05$ . $3073400+01$ $.3203517+05$ . $3855800+01$ $.1737164+05$ $.4510700+: 1$ $.2154000+05$ . $2394300+01$ . $1222593+05$

- . 38075J0+01 $.1247792+05$

$-.7650500+01$ . 2) $13919+1) \cdot$

- .19.27640+02 $.1412216+05$

-.1309370+02 $.3395560+04$

- .5577400+01 - $1323135+05$

-.2158420+02 - $1359760+05$ -. $2614190+02$ $.1156057+05$ - .2579390+02 . $1490300+05$

-.3553670+02 - $1271574+05$

-.3228450+02. . $1200816+0$ j -. $296.3620+02$ - $0039680+04$

-.1596620+02 - $y 793560+04$

-.5849c00+01 $.1099380+05$ $-.6553700+01$ . $1380445+05$ - $2343050+02$ . $2917340+04$ - $1315160+02$. $.2039480+05$ - $1628651+03$ $.1261239+C 5$
$.51510000-01$ - $1055792+03$ - 3+440000-01 $.5174210+0 !$ $.2230000-01$ $.7378180+02$ .3470000-01 - 14259E0+02 - 7400000-02 $-.5913940+02$ -.4370000-01 $-.5624550+02$ -.4630000-01 $-.7174870+02$ -.96600n 0-01 $-.5074770+02$ - .6830000-01 $-.9101250+0 \%$ - $1963 \mathrm{cod}+00$ $-.4274410+12$ -. $1002000+00$ $-.1469470+02$ - $5.320000-01$ - $1365890+0$ ? - 857 C C00-01 . $2105200+J 2$ $.1700000+00$ $.4485100+02$ - $4284003+00$ - $3524690+02$ $.4021000+00$ $.9217900+01$ - $12370(1)+0()$ $.3567250+02$ $.4796000+00$ . $370172 J+02$ - $520 x(0)+00$ - $3152110+02$ . $5732000+00$ $.3767550+02$ - 7857000+00 . $2580140+02$

$.7174(50+00$ $.2084730+0$ ? .6536couton $.8545700+01$ $.3543 \mathrm{j} 00+00$ . $2353100+01$ - $1300000+00$ - $2636600+01$ $.1456000+00$ -. $6155900+01$ $-.4533(00+0)$ -.306/800+01 -.2371c00+00 -. $302689 \mathrm{~J}+02$ -. $3478000+01$ $-.2343060+02$
$.2673644+04$

$.2349345+04$

$.522 .2543+04$

$.2096400+04$

$.2649032+04$

$.2519403+04$

$.1587407+04$

$.1122812+04$

$.1534376+04$

$.7014494+0.3$

$.2221545+03$

$-.2048839+03$

$-.3247789+03$

$-.6727730+03$

$-.5237021+03$

$-.1382675+03$

-.5350 isu. $3+0.3$

$-.55523061+03$

$-.4728143+03$

$-.56513 .32+0.3$

$-.4470132+03$

$-.3127 i, 73+03$

$-.1231344+03$

$-.3529550+02$

$-.3954030+02$

$.9233770+02$

$.4597170+0 ?$

$.4540207+03$

$.3514483+03$

$$
-.3769573+0.3
$$

$-.21034 .06+03$

$-.34124 ? 6+03$

$-.7236770+02$

$.4928729+03$

$.4687554+03$

$.1135118+04$

$.3028668+0 ?$

$.3291653+04$

$.1847979+04$

$.2833776+04$

$.1814639+04$

$.18830051+04$

$.3071606+04$

$.20676,13+04$

$.4846213+53$

$.1875453+04$

. $17938384+04$

$.1434365+104$

$.10 .32837+04$

$.124 ? 1 \neq 0+04$

. 32 $1\{0 ; 30+0.3$

. 325:41 $5+03$

$.3432270+1) 2$

$.983976 .20+02$

$-.2308442+04$

$-.114 \cdot 3293+03$

$-.1135052+04$

$-. \$ 784: 212+03$
$.3142 ? 71+32$

$.18 ? 4.300+02$

- $28445: 2+i 2$

- $50 \div 2500+.1$

$-.3990950+02$

$-.376,9680+92$

$-.750455011+02$

$-.5593670+02$

$-.1011202+03$

$-.320062:+0 ?$

$-.4357660+02$

$.7136: 20+0 ?$

$.14453 ?+03$

$.37 t 16 x i+03$

$. .30 / 5.344+03$

$.1138519+03$

- $4 \leq 006: 1+03$

. ร४⿻

$.0226405+0.3$

$.0393054+0.3$

$.9290153+03$

$.1113030+c 4$

$.+515037+03$

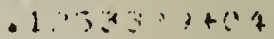

- Is bat at+

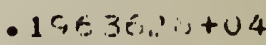

$.4571 \div 7:+63$

-32 $31012+04$

. 2051 ricit +04 
$.1551600+03$ $.1040994+0 j$ $.1792162+03$ - $1451103+05$ . $3239765+03$ - $1767162+05$ $.5074080+03$ . $4020000+05$ - $1624890+04$ $.7098000+04$ $.3701572+03$ $.2443520+04$ - $3613079+03$ $.1354624+05$ . $3823035+04$ $.3447360+04$ - $1704708+04$ $.3256220+04$ $.6961598+03$ $.5617630+04$ . $1179324+C 4$ $.2913130+04$ . $4803179+03$ $.7444980+04$ $.1062291+04$ $.4897870+04$ $.6436979+03$ - $1207272+05$ . $1458258+04$ . $4924660+04$ $.9413230+02$
$-.3221600+01$

-.2227050+02

-.3598900+01

$-.3392570+02$

$-.6356700+01$

$-.4364 \geqslant 20+02$

-. $9384300+01$

-.1168337+03

$-.2857780+02$

-. $1857210+02$

$-.5779200+01$

- $1195900+01$

- $1346000+01$

$.6166770+02$

- $1890877+03$ . $2827160+02$. - $9167570+02$ - $1396590+02$ - $7954630+02$ $.2416450+02$ $.1515549+03$ $.1105230+02$ - $1755064+03$ $.2476150+02$ $.4892138+03$ - $1512600+02$ - $3562247+03$ . $3442890+02$ - $9183255+03$ $.2275300+01$ $.574980 .3+03$

\begin{tabular}{|c|c|c|}
\hline $.3340444+03$ & $-.8351116+0.3$ & $.1706719+04$ \\
\hline $.5088639+03$ & $-.127216: 1+04$ & $.2361600+C 4$ \\
\hline $.6547047+03$ & $-.16 .36763+04$ & $.2825690+04$ \\
\hline $1753156+04$ & $-.4382894+04$ & $.7150316+04$ \\
\hline $.2785 t 14+03$ & $-.6964045+03$ & $.1047034+04$ \\
\hline - $1793330+02$ & $.4483340+02$ & $-.5874920+02$ \\
\hline $.9244161+03$ & $.2311069+04$ & $-.30283 t .3+04$ \\
\hline$-.42 .37870+03$ & $.10504: 31+04$ & $-.1388314+04$ \\
\hline$-.2092694+03$ & $.5231838+0.3$ & $-.6855575+03$ \\
\hline$-.3620637+03$ & $.9051795+0.3$ & $-.1150104+04$ \\
\hline$-.1654627+03$ & $.4136720+0.3$ & $-.5420427+03$ \\
\hline$-.37062+7+03$ & $.9265976+1) 3$ & $-.1214134+04$ \\
\hline$-.2263651+03$ & $.5659378+0.3$ & $\because .7415507+03$ \\
\hline $151772+03$ & $.1288003+04$ & $-.16 \$ 37669+04$ \\
\hline$-.3399910+02$ & $.8500390+02$ & $.1113750 j+03$ \\
\hline
\end{tabular}




\section{SUMMARY OF PROBLEM SOLUTIONS}

IDENTEETCATION HPI

a

SOURCE: I. Barrodale and F.D.K. Roberts, "An Improved Algorithm for Discrete $\ell$ Linear Approximation," SIAM J. Numer. Anal., 10 (1973), pp. 843-844.

CHARACTERISTICS

\# parameters

\# observations

5

rank full

otlier

\section{SOLUTTON}

objective function value

2.0

active constraints

1,3

solution vector

$0.5,0.5$

condition number 7

alternate solution vector yes

active constraints

solution vector $.75, .25$

condition number

8 
SOLRCE: R. R. Singleton, "A Method for Minimizing the Sum of Absolute Values of Deviations," Ann. Math. Stat., 11 (1940), p. 308.

\section{CHARACTERISTICS}

\# parameters_ 3 \# observations_ 17 rank ful1 other

\section{SOLUTION}

objective function value $10939 / 42$

active constraints $\quad 2,10,16$

solution vector $197 / 6,-29 / 14,-37 / 21$

condition number $1 \times 10^{2}$

alternate solution vector no

active constraints

solution vector

condition number 
SOURCE: R. H. Bartels, "CL1: A FORTRAN Subroutine for Unconstrained and Constrained L (LAR) Regression," Report to the National Bureau of Standards, November 1976, p. 16.

\section{CHARACTERISTICS}

非 parameters 2

\# observations 5 rank fu11 other

\section{SOIUTION}

$\begin{array}{ll}\text { objective function value } & \frac{11.0}{1,5} \\ \text { active constraints } & \frac{68.5,1.3}{\text { solution vector }} \\ \text { condition number } & \frac{2 \times 10^{2}}{2} \\ \text { alternate solution vector yes } \\ \text { active constraints } \frac{1,3}{63.25,1.45} \\ \text { solution vector } \\ \text { condition number }\end{array}$




\section{IDENTIEICATION HP4}

SOURCE: P. W. Holland, Educational Testing Service; see "Robust Test Problems for Robust Regression Problems," Proceedings of the Ninth Interface Symposium on Computer Science and Statistics, April 1976, pp. 99-110.

\section{CHARACTERISTICS}

\# parameters_6 \# observations_135 rank full

other Repeated rows in X matrix; Some variables qualitative $(-1,0,1)$

\section{SOLUTION}

objective function value $284269 / 504$

active constraints $\frac{5,20,37,39,68,110}{-\frac{-643}{1008}, \frac{-647}{504}, \frac{2573}{504}, \frac{-1459}{504}, \frac{43}{1008}, \frac{601}{28}}$
solution vector
condition number

alternate solution vector yes

active constraints $\quad 5,20,37,39,68,102$

solution vector $\frac{-643}{1008}, \frac{-647}{504}, \frac{869}{168}, \frac{-509}{168}, \frac{43}{1008}, \frac{1348}{63}$

condition number 


\section{IDENTIFICATION HP5}

SOURCE: U.S. Department of Labor, workload scheduling problem, 1967.

\section{CHARACTERISTICS}

非 parameters 8

\# observations 8 rank full other Does not contain a column of 1's

\section{SOLUTION}

objective function value 0.0

active constraints $1,2,3,4,5,6,7,8$

$126 . \overline{3588,-3.378714,1.022894,16.35045}$, solution vector $-45.39628,33.13378,-34.60532, .4174025$

condition number

$3 \times 10^{3}$

alternate solution vector

no

active constraints

solution vector

condition number 
SOI,PCE: Modification of problem from I. Barrodale and A. Young, "Algorithms for Best $L_{1}$ and $L_{\infty}$ Linear Approximations on a Discrete Set," Numer. Math., 8 (1966), p. 298.

\section{CHARACTERISTICS}

\# parameters_ 2 \# observations_ 6 rank full other

\section{SOLUTION}

objective function value $11 / 15$

active constraints 1,4

solution vector $76 / 5,-151 / 30$

condition number

2

alternate solution vector no

active constraints

solution vector

condition number 
TIENTIFICITION HP7

SÚURCE: K. Spyropoulos, E. Kiountouzis and A. Young, "Discrete Approximation in the $\mathrm{L}_{1}$ Norm," Computer J., 16 (1973), p. 184.

CHARACIERISTICS

\# parameters___ 2 \# observations_ 7 ___ank full other degeneracy

\section{SCL.VTION}

objectsive function value 16.0

active constraints Any two distinct indices from $\{1,3,4,5,7\}$

solutiou vector

conditior jumber $\ldots+7 \times 10^{1}$ to $5 \times 10^{2}$

alternate solution vector no

active constraints

sulution vector

conditson number 


\section{IDENTIFICATION HP8}

SOURCE: I. Barrodale and F. D. K. Roberts, "An Improved Algorithm for Discrete \& Linear Approximation," University of Wisconsin, Mathematics Research Center, MRC Technical Summary Report \#1172, January 1972.

\section{CHARACTERISTICS}

\# parameters

\# observations

201

rank full

other

\section{SOLUTION}

objective function value

21.81558

active constraints 107

solution vector .1023800

condition number 1

alternate solution vector no

active constraints

solution vector

condition number 


\section{IDENTIFICATION HP9}

SOURCE: I. Barrodale and F. D. K. Roberts, "An Improved Algorithm for Discrete \& Linear Approximation," University of Wisconsin, Mathematics Research Center, MRC Technical Summary Report \#1172, January 1972.

\section{CHARACTERISTICS}

\# parameters

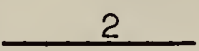

\# observations 201

rank full

other

\section{SOLUTION}

objective function value 9.271694

active constraints 94,169

solution vector $.3435676,-.1044933$

condition number 13

alternate solution vector no active constraints solution vector condition number 
IDENTIFICATION HPIO

SOURCE: I. Barrodale and F. D. K. Roberts, "An Improved Algorithm for Discrete \& Linear Approximation," University of Wisconsin, Mathematics Research Center, MRC Technical Summary Report \#1172, January 1972.

\section{CHARACTERISTICS}

\# parameters 3 \# observations 201 rank full other

\section{SOLUTION}

objective function value 9.078925

active constraints $30,101,172$ solution vector $\quad .3967848,-.1625170, .01282731$

condition number $5 \times 10^{1}$

alternate solution vector no active constraints solution vector condition number 


\section{IDENTIFICATION HPII}

SOURCE: I. Barrodale and F. D. K. Roberts, "An Improved Algorithm for Discrete $\ell$ Linear Approximation," University of Wisconsin, Mathematics Research Center, MRC Technical Summary Report \#1172, January 1972.

\section{CHARACTERISTICS}

\# parameters 4 \# observations

rank full

other

\section{SOLUTION}

objective function value

4.640714

active constraints $20,70,132,182$

solution vector $.1640695, .3230841,-.2446924, .03922143$

condition number $4 \times 10^{2}$

alternate solution vector no active constraints : solution vector condition number 
SOLRCE: I. Barrodale and F.D.K. Roberts, "An Improved Algorithm for Discrete $\ell$ Linear Approximation," University of Wisconsin, Mathematics Research Center, MRC Technical Summary Report 非1172, January 1972 .

\section{CHARACTERISTICS}

\# parameters_ 5 非 observations_201_ rank full

othe:

\section{SOLUTION}

objective function value 1.055658

active constraints $14,51,101,151,188$

solution vector $.03041441, .8248866,-.7469379, .2235657,-.02236884$

condition number $4 \times 10^{3}$

alternate solution vector no

active constraints

solution vector

condition number 
SOURCE: I. Barrodale and F.D.K. Roberts, "An Improved Algortthm for Discrete $\ell$ Linear Approximation," University of Wisconsin, Mathematics Research Center, MRC Technical Summary Report 非1172, January 1972 .

\section{CHARACTERISTICS}

\# parameters 6 \# observations 201 rank ful1 other

\section{SOLUTION}

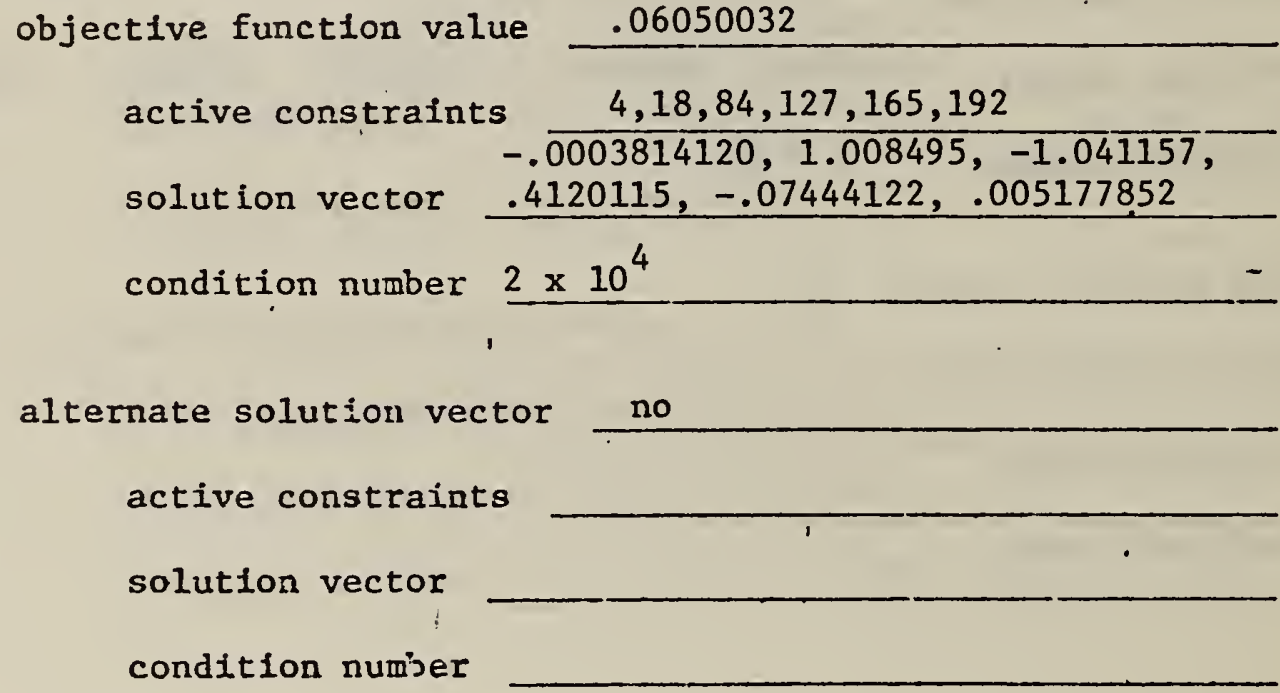


SOURCE: I. Barrodale and F.D.K. Roberts, "An Improved Algorithm for Discrete $\ell_{1}$ Linear Approximation," University of Wisconsin, Mathematics Research Center, MRC Technical Summary Report \#1172, January 1972 .

\section{CHARACTERISTICS}

\# parameters_ 7 \# observations_ 201 rank full
other

\section{SOLUTION}

objective function value .03471234

$$
\begin{array}{ll}
\text { active constraints } \frac{8,30,63,101,139,172,194}{} & -.002115490,1.024030,-1.078237, .4485926, \\
\text { solution vector }-.09159510, .008979690,-.0003203885 \\
\text { condition number } 4 \times 10^{5}
\end{array}
$$

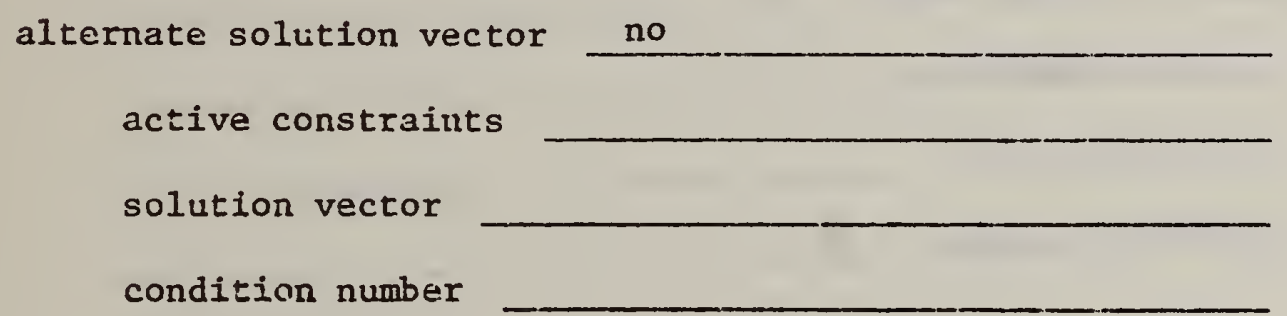


SOURCE: I. Barrodale and F.D.K. Roberts, "An Improved Algorithm for Discrete $\ell_{1}$ Linear Approximation," University of Wisconsin, Mathematics Research Center, MRC Technical Summary Report 非1172, January 1972.

\section{CHARACTERISTICS}

\# parameters 6

\# observations 100

rank full

other

\section{SOLUTION}

objective function value $\quad 3.877737$

active constraints $19,35,46,70,82,97$

$1.129434,-.7975623,-1.628313$

solution vector $1.316736,-.1082232, .1593164$

condition number $\quad 4 \times 10^{2}$

alternate solution vector no

active constraints

solution vector

condition number 
SOURCE: I. Barrodale and F.D.K. Roberts, "An Improved Algorithm for Discrete $\ell_{1}$ Linear Approximation," University of Wisconsin, Mathematics Research Center, MRC Technical Summary Report \#1172, January 1972 .

\section{CHARACTERISTICS}

\# parameter 8

\# observations

51

rank full

other

\section{SOLUTION}

objective function value .05340562

active constraints $1,4,8,20,28,35,43,47$

$0 ., 6.262394,-44.95925,141.1803$,

solution vector $-136.6328,-3.195617,-1.100961,-.1572059$

condition number $1 \times 10^{5}$

alternate solution vector no

active constraints

sclutior vector

condition number 
SOURCE: I. Barrodale and F.D.K. Roberts, "An Improved Algorithm for Discrete $\ell$ Linear Approximation," University of Wisconsin, Mathematics Research Center, MRC Technical Summary Report \#1172, January 1972 .

\section{CHARACTERISTICS}

\# parameters_ 6 \# observations_23 rank full

other degeneracy

\section{SOLUTION}

objective function value

Any six distinct indices from

active constraints $\{2,7,11,15,20,22,23\}$

solution vector $0 . . .9995966,0.2,-.1652754,0 ., .007352267$

condition number $1 \times 10^{5}$ to $4 \times 10^{5}$

alternate solution vector

no

active constraints

solutiun vector

condition number 
SOU.RCE: I. Barrodale and F.D.K. Roberts, "An Improved Algorithm for Discrete $\ell_{1}$ Linear Approximation," University of Wisconsin, Mathematics Research Center, MRC Technical Summary Report 非1172, January 1972 .

\section{CHARACTERISTICS}

\# parameters_ 6 \# observations_53 rank full

other degeneracy

\section{SOLUTION}

objective function value .004344250 Any six distinct indices from active constraints $\{5,15,26,37,47,52,53\}$

solution vector $0 ., .9995389,0 .,-.1652942,0 ., .007358741$

condition number $1 \times 10^{5}$ to $3 \times 10^{5}$

a.ternate solution vector no active constraints solution vector condition number 
SOURCE: I. Barrodale and F.D.K. Roberts, "An Improved Algorithm for Discrete $\ell$ Linear Approximation," University of Wisconsin, Mathematics Research Center, MRC Technical Summary Report 非1172, January 1972 .

\section{CHARACTERISTICS}

\# parameters 6 \# observations 103 rank full

other degeneracy

\section{SOLUTION}

cbjective function value active constraints .008568850

Any six distinct indices from $\{10,29,51,73,22,102,103\}$

solution vector $0 . . .9995529,0 .,-.1653271,0 ., .007366283$ condition number $1 \times 10^{5}$ to $4 \times 10^{5}$

alcernate solution vector no active constraints solution vestor condition number 
SOLPCE: I. Barrodale and F.D.K. Roberts, "An Improved Algorithm for Discrete \& Linear Approximation," University of Wisconsin, Mathematics Research Center, MRC Technical Summary Report \#1172, January 1972 .

\section{CHARACTERISTICS}

\# parameters_ 6 \# observations_ 203 rank full
other_degeneracy

\section{SOLUTION}

$$
\begin{aligned}
& \text { objective function value } \\
& .01694253 \\
& \text { Any six distinct indices from } \\
& \text { active constraints }\{18,57,101,145,184,202,203\} \\
& \text { solution vector } 0 ., .9995427,0 .,-.1653031,0 ., .007360762 \\
& \text { condition number } 1 \times 10^{5} \text { to } 4 \times 10^{5}
\end{aligned}
$$

alternate solution vector

no

active constrairts

solution vector

condition number 
SOURCE: I. Barrodale and F.D.K. Roberts, "An Improved Algorithm for Discrete $\ell_{1}$ Linear Approximation," University of Wisconsin, Mathematics Research Center, MRC Technical Summary Report \#1172, January 1972.

\section{CHARACTERISTICS}

\# parameters_ 11 \# observations_21_ rank_ful1

other

\section{SOLUTION}

objective function value .1057780

active constraints $1,3,4,6,7,12,15,16,18,20,21$ $602.3115,-781.8188,94.48467,-463.9671,-1326.421$, solution vector $1248.389,605.0711,-612.3761,98.38093,82.99064$,

condition number $-72.82688$ $4 \times 10^{6}$

alternate solution vector ne active constraints solution vector condition number 
SOURCE : I. Barrodale and F.D.K. Roberts, "An Improved Algorithm for Discrete $\ell$ Linear Approximation," University of Wisconsin, Mathematics Research Center, MRC Technical Summary Report 非172, January 1972 .

\section{CHARACTERISTICS}

\# parameters_ 11 \# observations_ 51 rank full other

\section{SOLUTION}

objective function value .2667665

active constraints $1,2,5,12,13,17,23,30,39,45,50$ $49.79747,-117.3993,328.0159,-425.9049,-627.8460$ solution vector $642.0325,213.0777,-283.7580,76.16677,35.84595$, $-38.21172$

condition number $5 \times 10^{6}$

diternate solution vector no active constraints solution vector condition number 
SOURCE: I. Barrodale and F.D.K. Roberts, "An Improved Algorithm for Discrete $\ell_{1}$ Linear Approximation," University of Wisconsin, Mathematics Research Center, MRC Technical Summary Report \#1172, January 1972 .

\section{CHARACTERISTICS}

\# parameters_ 11 \# observations_ 101 rank full other

\section{SOLUTION}

objective function value .5450632

active constraints $2,11,16,28,33,44,60,71,77,82,101$

$178.6954,-242.1546,114.2572,-254.2264,-525.6558$

solution vector $527.5312,207.5384,-247.8918,52.52677,32.13306$,

condition number $-33.36465$

$$
\begin{array}{r}
-33.36465 \\
2 \times 10^{6} \\
\hline
\end{array}
$$

alternate solution vector

no

active constraints

solution vector

condition number 
SOURCE: I. Barrodale and F.D.K. Roberts, "An Improved Algorithm for Discrete $\ell$ Linear Approximation," University of Wisconsin, Mathematics Research Center, MRC Technical Summary Report 非1172, January 1972 .

\section{CLARACTERISTICS}

\# parameters 11 \# observations_201 rank full

other

\section{SOLLTION}

objective function value 1.129209

active constraints $3,9,17,24,30,53,117,155,160,181,198$

$403.3163,-277.9515,-269.0950,-210.9572,-286.9815$

solution vector $497.1644,102.2011,-234.4954,88.33653,29.47814$, $-36.77963$

condition number

$4 \times 10^{6}$

alternate solution vector no

active constraints

solution vector

condition number 
SOlPCE: I. Barrodale and F.D.K. Roberts, "An Improved Algorithm for Discrete $\ell_{1}$ Linear Approximation," University of Wisconsin, Mathematics Research Center, MRC Technical Summary Report. 非1172, January 1972 .

\section{CHARACTERISTICS}

\# parameters_ 5 \# observatiors_ 15 rank

other Does not contain a column of $1^{\prime}$ s

\section{SOLUTION}

objective function value $1677 / 49$

active constraints

solution vector $25 / 49,10 / 49,4 / 49,0,0$

condition number

4

alternate solution vector yes

active constraints $4,7,11$

solution vector $29 / 49,14 / 49,0,0,-4 / 49$

condicion number 3 
SOGRC: I. Barrodale and F.D.K. Roberts, "An Improved Algorithm for Discrete $\ell_{1}$ Linear Approximation," University of Wisconsin, Mathematics Research Center, MRC Technical Summary Report 非1172, January 1972 .

CHARACTERISTICS

\# parameters_ 5 if observations 51 rank full_

other

\section{SOLUTION}

objective function value

16.73596

active constraints $4,13,26,38,51$ $.2884646,-5.961055,41.81349,-82.04005$, solution vector 54.89915

condition number $1 \times 10^{3}$

alternate solution vector

no

active constraints

solution vector

condition number 
Iurerergated HP27

SOUice: Treasury Department, spline-fitting problem, 1976.

\section{CHARACTERISTICS}

\# parameters 6

\# observations

72

rank fuli

other

\section{SOLUTION}

objective function value 11948.75

active constraints $11,34,52,61,66,71$

$5.399219,6.926597,7.180670,7.532025$, solution vector $7.718703,8.343703$

condition number $\ldots 4 \times 10^{1}$

alternate solution vector no

active constraints

solution vector

concition number 


\section{REFERENCES}

[1] Abdelmalek, N. N., "An Efficient Method for the Discrete Linear $L_{1}$ Approximation Problem," Mathematics of Computation, Vol. 29 (1975), 844850.

[2] Armstrong, R. D. and Frome, E. L., "A Comparison of Two Algorithms for Absolute Deviation Curve Fitting," Journal of the American Statistical Association, Vo1. 71, No. 354 (1976), 328-330.

[3] Barrodale, I., "L $\mathrm{L}_{1}$ Approximation and the Analysis of Data," Applied Statistics, Vol. 17 (1968), 51-57.

[4] Barrodale, I. and Roberts, F. D. K., "An Improved Algorithm for Discrete l1 Linear Approximation," SIAM Journal on Numerical Analysis, Vo1. 10, No. 5 (1973), 839-848.

[5] Barrodale, I. and Roberts, F. D. K., "Solution of an Overdetermined System of Equations in the $\ell_{1}$ Norm," Communications of the Association for Computing Machinery, Vol. 17 (197.4), 319-320.

[6] Bartels, R. H., Conn, A. R., and Sinclair, J. W., "Minimization Techniques for Piecewlse Differentiable Functions: The $\ell_{1}$ Solution to an Overdetermined Linear Systen," SIAM Journal on Numerical Analysis, Vol. 15, No. 2 (1978), 224-241.

[7] Dongarra, J. J., Moler, C. B., Bunch, J. R., and Stewart, G. W., "LINPACK Users' Guide," Soclety for Industrial and Applied Mathematics, Philadelphia, 1979.

[8] Domich, P., Lawrence, J., and Shier, D., "Generators for Discrete Polynomial L 1 Approximation Problems," Journal of Research, National Bureau of Standards, Vo1. 84, No. 6 (1979), to appear.

[9] Draper, N. R. and Smith, H., Applied Regression Analysis, John Wiley \& Sons, New York, 1966.

[10] Gentle, J. E., "Least Absolute Values Estimation: An Introduction," Communications in Statistics, Vol. B6, No. 4 (1977), 313-328.

[11] Gentle, J. E.., Kennedy, W. J., and Sposito, V. A., "On Least Absolute Values Estimation," Communications in Statistics, Vol. A6, No. 9 (1977), 839-845.

[12] Gilsinn, J., Hoffman, K., Jackson, R. H. F., Leyendecker, E., Saunders, P., and Shier, D., "Methodology and Analysis for Comparing Discrete Linear $\mathrm{L}_{1}$ Approximation Codes," Communications in Statistics, Vol. B6, No. $4(1977), 399-413$.

[13] Hampel, F. R., "A General Qualitative Definition of Robustness," Annals of Mathematical Statistics, Vol. 42 (1971), 1887-1896. 
[14] Harter, H. L., "Nonuniqueness of Least Absolute Values Regression," Communications in Statstics, Vo1. A6, No. 9 (1977), 829-383.

[15] Hillier, F. S. and Lieberman, G. J., Introduction to Operations Research, Second Edition, Holde-Day, San Francisco, 1974.

[16] Hoffman, K. and Shier, D., "A Test Problem Generator for Discrete Linear $\mathrm{L}_{1}$ Approximation Problems," Transactions on Mathematical Sof tware, to appear.

[17] Hollard, P. W., "Robust Test Problems for Robust Regression Programs," Proceedings of the Ninth Interface Symposium on Computer Science and Statistics, D. C. Hoaglin and R. E. Welsch (eds.), April 1976, 99-105.

[18] Johnston, J., Econometric Methods, Second Edition, McGraw-Hi11, New York, 1972.

[19] Kennedy, W. J., Gentle, J. E., and Sposito, V. A., "A Computer Oriented Method for Generating Test Problems for $\mathrm{L}_{1}$ Regression," Communications in Statistics, Vol. B6, No. 1 (1977), 21-27.

[20] Ortega, J. M., Numerical Analysis, A Second Course, Academic Press, New York, 1972.

[21] Rao, C. R., Linear Statistical Inference and its Applications, John Wiley \& Sons, New York, 1965.

[22] Rice, J. R. and White, J. S., "Norms for Smoothing and Estimation," SLAM Review, Vo1. 6, No. $3(1964), 243-256$.

[23] Rosenberg, B. and Carlson, D., "A Simple Approximation of the Sampling Distribution of Least Absolute Residuals Regression Estimates," Communications in Statistics, Vo1. B6, No. 4 (1977), 421-437.

[24] Schlossmacher, E. J., "An Iterative Technique for Absolute Deviations Curve Fitting," Journal of the American Statistical Association, Vol. 68 , No. 344 (1973), 857-859.

[25] Spyropoulos, K., Kiountouzis, E., and Young, A., "Discrete Approximation in the $L_{1}$ Norm," Computer Journal, Vo1. 16, No. 2 (1973), 180-186.

[26] Tukey, J. W., "The Future of Data Analysis," Annals of Mathematical Statistics, Vol. 33 (1962), 1-67. 
MBS-114A (REV. 9-78)

\begin{tabular}{|c|c|}
\hline U.S. DEPT. OF COMM. & 1. PUBLICATION OR REPORT NO. \\
BIBLIOGRAPHIC DATA \\
SHEET
\end{tabular}

4. TITLE AND SUBTITLE

A Collection of Test Problems for Discrete Linear $\mathrm{L}_{1}$ Data Fitting

7. $A U T H O R(S)$

D. R. Shier, S. J. Neupauer, P. B. Saunders

9. PERFORMING ORGANIZATION NAME AND ADDRESS

NATIONAL BUREAU OF STANDARDS

DEPARTMENT OF COMMERCE

WASHINGTON, DC 20234

12. SPONSORING ORGANIZATION NAME AND COMPLETE ADDRESS (Streot, City, State, ZIP) Operations Research Division

Center for Applied Mathematics

National Bureau of Standards

Washington, DC 20234

15. SUPPLEMENTARY NOTES

Document describes a computer program; SF-185, FIPS Software Summary, is attached.

16. ABSTRACT (A 200-word or less tactual summary of most significant information. If document includes a significant bibliography or literature survey, mention it hero.)

This document assembles 27 test problems representing a variety of examples in which least absolute deviation (or $\mathrm{L}_{1}$ ) data fitting has been used. The problems were collected from the literature, From the authors of several $\mathrm{L}_{1}$ approximation codes, and from examples encountered in practice. Optimal $\mathrm{L}_{1}$ solutions to these problems (objective function value and solution vectort have been obtained using a double-precision computer code designed for checking the Kuhn-Tucker conditions and for performing an accurate reinversion of the optimal basis. Special problem characteristics such as alternative optima, degeneracy, and rank loss are also noted. This set of test problems has proven useful in evaluating and improving the performance of $\mathrm{L}_{1}$ codes as well as in suggesting types of problem structures that might be mimicked by problem generators.

17. KEY WORDS (six to twelve entries; alphabetical order; capitalize only the firat letter of the first koy word unleas a proper name; soparated by semicolons)

Algorithm testing; approximation; curve fitting; least absolute deviation; polynomial approximation; spline fitting; test data; test problems.

\begin{tabular}{|l|l|}
\hline $\begin{array}{l}\text { 19. SECURITY CLASS } \\
\text { (THIS REPORT) } \\
\text { UNCLASSIFIED }\end{array}$ & $\begin{array}{l}\text { 21. NO. OF } \\
\text { PRINTED PAGES }\end{array}$ \\
\hline $\begin{array}{l}\text { 20. SECURITY CLASS } \\
\text { (THIS PAGE) } \\
\text { UNCLASSIFIED }\end{array}$ & 22. PriCe \\
\hline
\end{tabular}




